\title{
Q
}

Anno di fondazione 1824

\section{Naturalità e Vulnerabilità Ambientale nei Siti Natura 2000 che insistono sui corridoi ecologici del versante jonico calabrese. Caso studio SIC Foce Neto ${ }^{\dagger}$}

\author{
Arcidiacono C. [1], Costa R.M.S. [2], Grasso I. [3], Ragusa M.A. [4], \\ Rapicavoli R. V. [5], Seminara M. [6], Veneziano V. [7]
}

[1] Dipartimento di Agricoltura, Alimentazione e Ambiente, Università degli Studi di Catania

[2] Dipartimento di Scienze Biologiche, Geologiche, Ambientali, Sez. Botanica,

Università degli Studi di Catania

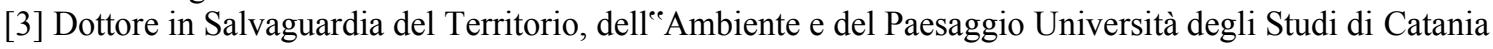

[4] Dipartimento di Matematica ed Informatica, Università degli Studi di Catania

[5] Biologa

[6] Informatico

[7] Biologo, PhD

\section{Riassunto}

La regione Calabria vanta 179 SIC (Siti di Interesse Comunitario) e 6 ZPS (Zone di Protezione Speciale). La carenza di studi sullo stato di conservazione e qualità ambientale dei suddetti siti (naturalità, artificialità, sensibilità, vulnerabilità e rischio) ha indotto gli AA. a caratterizzare i 10 SIC che insistono sui corridoi ecologici del versante jonico calabrese. Attraverso l'ILC (Index of Landscape Conservation) e l'ESAi (Environmentally Sensitive Areas Index), è stata condotta un "eanalisi ambientale finalizzata alla definizione, individuazione e valutazione dei valori e delle criticità. Muovendo dalla Carta d'Uso del Suolo (CLC) sono state realizzate, quali carte derivate, la Carta della Naturalità-Artificialità e la Carta della Sensibilità Ambientale dei suddetti siti. Lo studio chiude con un approfondimento metodologico sulle opportune modalità da adottare per caratterizzare un SIC; modalità suggerite dalle analisi ambientali eseguite soprattutto sul SIC Foce Neto adottando una capillare indagine geobotanica condotta con metodo fitosociologico.

Parole chiave: analisi ambientale, impatti, naturalità, artificialità, CLC, variazione superfici, ILC (Index of Landscape Conservation), Data Base, MEDALUS, suolo, fertilità, vulnerabilità, sensibilità, Crotone, SIT, geobotanica, fiume Neto, SIC, ZPS.

\section{Summary}

\section{Naturalness and environmental vulnerability in the Nature 2000 sites that cover the ecological corridors of the Calabria's Ionian side. SIC Foce Neto case study}

The Calabria region boasts 179 Sites of Community Interest (SIC) and 6 Special Protection Areas (SPAs). The lack of studies on the state of conservation and environmental quality of these sites (naturalness, artificiality, sensitivity, vulnerability and risk) has led the AA. to characterize the 10 SIC that insist on the ecological corridors of the Ionian Calabrian side. Through the Index of Landscape Conservation (ILC) and Environmentally Sensitive Areas Index (ESAi), an Environmental Analysis was conducted aimed at defining, identifying and assessing values and criticalities. Starting from the Land Use Map (CLC), the Map of 
Naturalness-Artificiality and the Map of Environmental Sensitivity of the afore mentioned sites have been created, such as derived maps. This study is concluded with a methodological study on the appropriate methods that should be taken to characterize a SIC thanks to the analysis, made before, on SIC Neto, addressed with phytosociological method.

Key words: Environmental Analysis, Impacts, Naturality, Artificiality, CLC, surface variation, Structural Indexes, ILC (Index of Landscape Conservation), CLC, Data Base, MEDALUS, soil, fertility, vulnerability, sensitivity, Crotone, SIT, geobotany, Neto river, SIC, ZPS.

\section{Introduzione}

Negli anni 60 matura il concetto di Ambiente, da semplice contenitore a complessa realtà, dove interagiscono innumerevoli elementi fisici e biologici ma, soprattutto, che la risorsa è limitata. Mentre nel passato il degrado ambientale non era oggetto di valutazione, solo di recente la società ha scoperto i costi occulti; costi che iniziano a entrare nella contabilità sotto forma di Valutazioni Ambientali (VIA, VAS, VIncA, VIS, AIA) e di quantificazioni del Capitale Naturale (CCN, 2017). Purtroppo gli studi del passato, in quanto commissionati da lobby industriali, tendevano a dimostrare il ridotto impatto delle varie azioni con la finalità di sensibilizzare i cittadini ad accettare il rapporto costo-benefici. Questo retroterra storico ha determinato una crescente conflittualità fra le popolazioni da un lato e le amministrazioni pubbliche e grandi aziende dall'altro con conseguenze, spesso, di paralisi decisionali.

La Conservazione dell'Ambiente è oggi un fattore non eludibile nella produzione di servizi.

Assumere decisioni di Tutela Ambientale significa disporre di adeguate informazioni, pesate a seconda dell'importanza che rivestono. La Qualità Ambientale ai fini conservazionistici viene quasi sempre analizzata attraverso lo studio della Vegetazione, del suo dinamismo ossia dalla distanza che intercorre tra Vegetazione Attuale e Vegetazione Potenziale. Nella sua forma più semplice la Vegetazione viene distinta in tre grandi categorie (vedasi Bibliografia):

- $\quad$ naturale - quando non esiste alterazione nella struttura e nella composizione floristica;

- $\quad$ seminaturale - quando risulta modificata nella struttura ma non nella composizione;

- $\quad$ artificiale - quando l'alterazione interessa sia la struttura che la composizione.

Naturalità - Valori di Naturalità elevata sono ormai rare e coincidono con tipi di vegetazione prossimi allo stadio climax. Valori di Naturalità medio-alta si riferiscono a cenosi prevalentemente forestali che conservano una coerenza floristica e strutturale. Valori di Naturalità media si riscontrano in quelle cenosi che hanno un'alterazione strutturale (es. cespuglieti di ricostituzione e praterie secondarie). Valori di Naturalità debole sono propri di tipi vegetazionali fortemente alterati anche nella composizione floristica ma ancora non completamente artificiali (es. rimboschimenti dove sia in atto un processo di colonizzazione da parte di specie appartenenti alla flora locale). Nei valori di Naturalità molto debole rientrano le formazioni totalmente artificiali (es. seminativi e rimboschimenti).

Vari autori si sono cimentati nello stimare il Valore Ambientale muovendo da parametri biologici desunti prevalentemente da categorie dell'uso del suolo o del cambiamento del suolo. I tematismi ambientali scelti a tal fine sono, soprattutto, la naturalità, il valore biogeografico, la diversità biologica; la rarità e l'impatto umano sono quelli più adottati. 
Per poter assegnare un Valore Ambientale ad una parcella di territorio è fondamentale caratterizzare il grado di Naturalità ossia l'entità di perturbazione da parte antropica.

Si va da scale caratterizzate da pochi gradi di Naturalità - assegnate a un territorio in base alla valutazione soprattutto della flora, della vegetazione e dell'antropizzazione - a scale più complesse dove entrano in gioco i temi della rarità, diversità, ritorno alla Naturalità, antropizzazione. Infine esistono le scale ad approccio paesaggistico che assegnano un valore di Naturalità ad una tessera di territorio valutando i valori percentuali di copertura di ogni unità al fine di perseguire una valutazione complessiva dell'area.

La rappresentazione più classica della Naturalità di un territorio è di norma restituita da una carta tematica derivata dalla Carta della Vegetazione. La Carta deve riportare il livello di qualità di un territorio capace di auto-conservarsi, livello che viene misurato in gradi di Naturalità o di Artificialità. E' altresì opportuno confrontare le Carte della Vegetazione Attuale e Potenziale al fine di interpretare la distanza che intercorre dalla vegetazione durevole.

Si sottolinea che una Carta della Naturalità diventa uno strumento di stima rigorosa della qualità di un territorio solo e soltanto quando è desunta da una Carta della Vegetazione e non da una Carta dell'Uso del Suolo, come ancora oggi molti si ostinano ad adottare (quest'ultima scelta è dettata da economicità di approccio e dal non dover mettere in campo alte competenze professionali - es. geobotanici).

Vulnerabilità Ambientale - (Sensibilità o Fragilità) è una variabile che descrive l'attitudine di un'unità ambientale a subire degradi in conseguenza di pressioni esterne. In campo vegetazionale le cenosi forestali con elevato valore di Naturalità spesso sono molto vulnerabili in quanto vivono in ambienti limitanti per qualche fattore ecologico. Viceversa quelle cenosi costituite da specie con elevata capacità di rigenerazione o propagazione hanno una sensibilità minore in quanto possono ricostituirsi facilmente in seguito ad impatti.

Rete Natura 2000 - Istituita ai sensi della Direttiva 92/43/CEE Habitat per tutelare gli habitat naturali e le specie di flora e fauna minacciati o rari in tutto il territorio dell'Unione; rappresenta il principale strumento di politica dell'Unione Europea per la Conservazione della Biodiversità. È costituita dai Siti di Interesse Comunitario (SIC), che evolvono in Zone Speciali di Conservazione (ZSC), e dalle Zone di Protezione Speciale (ZPS) istituite ai sensi della Direttiva 2009/147/CE Uccelli per la conservazione degli uccelli selvatici. L'obiettivo della Direttiva è conservare non solo gli habitat naturali ma anche quelli semi-naturali (come le aree ad agricoltura tradizionale, $\mathrm{i}$ pascoli, ecc.).

In Italia i SIC, le ZSC e le ZPS coprono complessivamente circa il 19\% del territorio nazionale e quasi il $4 \%$ di quello marino.

La Società Botanica Italiana (SBI) ha realizzato il Manuale di interpretazione degli habitat adattato alla realtà italiana (MATTM, 2010) per facilitare l'identificazione di quegli habitat la cui descrizione nel Manuale europeo (Biondi et al., 2009 ) non risulta adeguata al contesto nazionale.

In base all'art. 6 del nuovo DPR 120/2003, comma 1, nella pianificazione e programmazione territoriale si deve tenere conto delle valenze naturalistico-ambientali dei proposti SIC, SIC e ZPS 
per evitare che vengano approvati strumenti di gestione territoriale in conflitto con le esigenze di conservazione degli habitat e delle specie di interesse comunitario. Il comma 2 dello stesso art. 6 stabilisce che è sottoposto a Valutazione di Incidenza Ambientale (VIncA) qualunque Piano od Opera non direttamente connesso e necessario al mantenimento in uno stato di conservazione soddisfacente delle specie e degli habitat presenti in un sito Natura 2000. In ambito nazionale, la VIncA viene disciplinata dall'art. 6 del DPR 12 marzo 2003, n. 120, (G.U. n. 124 del 30 maggio 2003) che ha sostituito l'art.5 del DPR 8 settembre 1997, n. 357.

La Regione Calabria (delibera n.462 del 12.11.2015) ha istituito 178 SIC che tutelano 70.197 ha terrestri e una superficie marina di 20.251 ha (Fig.1).

La carenza di studi sullo stato di conservazione e qualità ambientale dei suddetti siti (naturalità, artificialità, sensibilità, vulnerabilità e rischio) ha indotto gli AA. a caratterizzare i 10 SIC che insistono sui corridoi ecologici del versante jonico calabrese.

La finalità dello studio è un approfondimento metodologico sulle modalità di caratterizzazione di un SIC.

A parere degli AA. una proposta SIC dovrebbe essere accompagnata sin dalle fasi iniziali da una cartografia a grande scala $(1: 10.000)$ che discende da una analisi ambientale geobotanica condotta attraverso rilievi fitosociologici georiferiti (punti verità). Si eviterebbero errori di interpretazione (vedasi le risultanze del caso studio), limitando le crescenti conflittualità di quanti vedono mortificati le potenzialità dei propri appezzamenti a causa di indagini approssimative sul piano del rigore scientifico.

\section{Materiali e Metodi}

Molte delle attività umane hanno contribuito alla perdita della qualità dei paesaggi - complessi sistemi eterogenei dal punto di vista degli ecosistemi, dei fattori biotici, abiotici e di uso del suolo - minacciando la biodiversità, la stabilità e la continuità di tali ecosistemi. Al fine di rendere compatibile la pianificazione delle attività umane con la conservazione della biodiversità e della naturalità del territorio, ci s'interroga su come conservare i paesaggi di qualità e sui metodi e parametri da utilizzare per arrivare a misurare il valore del paesaggio da tutelare.

Molti autori hanno rivolto le loro ricerche all'analisi della biodiversità e alla valutazione della sua perdita, in termini di specie e di habitat, definendo indici di Qualità Ambientale partendo da dati floristico-vegetazionali (Lausi et al., 1978; Poldini, Pertot, 1989; Ferrari et al., 2000; Biondi \& Colosi, 2005; Cerabolini et al., 2007).

Per stimare la Naturalità-Artificialità gli AA. hanno preliminarmente messo in correlazione le unità vegetazionali individuate con il sistema di classificazione degli habitat secondo la nomenclatura CORINE Biotopes e secondo la Direttiva Habitat.

La Qualità degli Habitat è stata definita mediante due indicatori in grado di esprimere il Valore Naturale dell'Habitat e il Livello di Conservazione. 


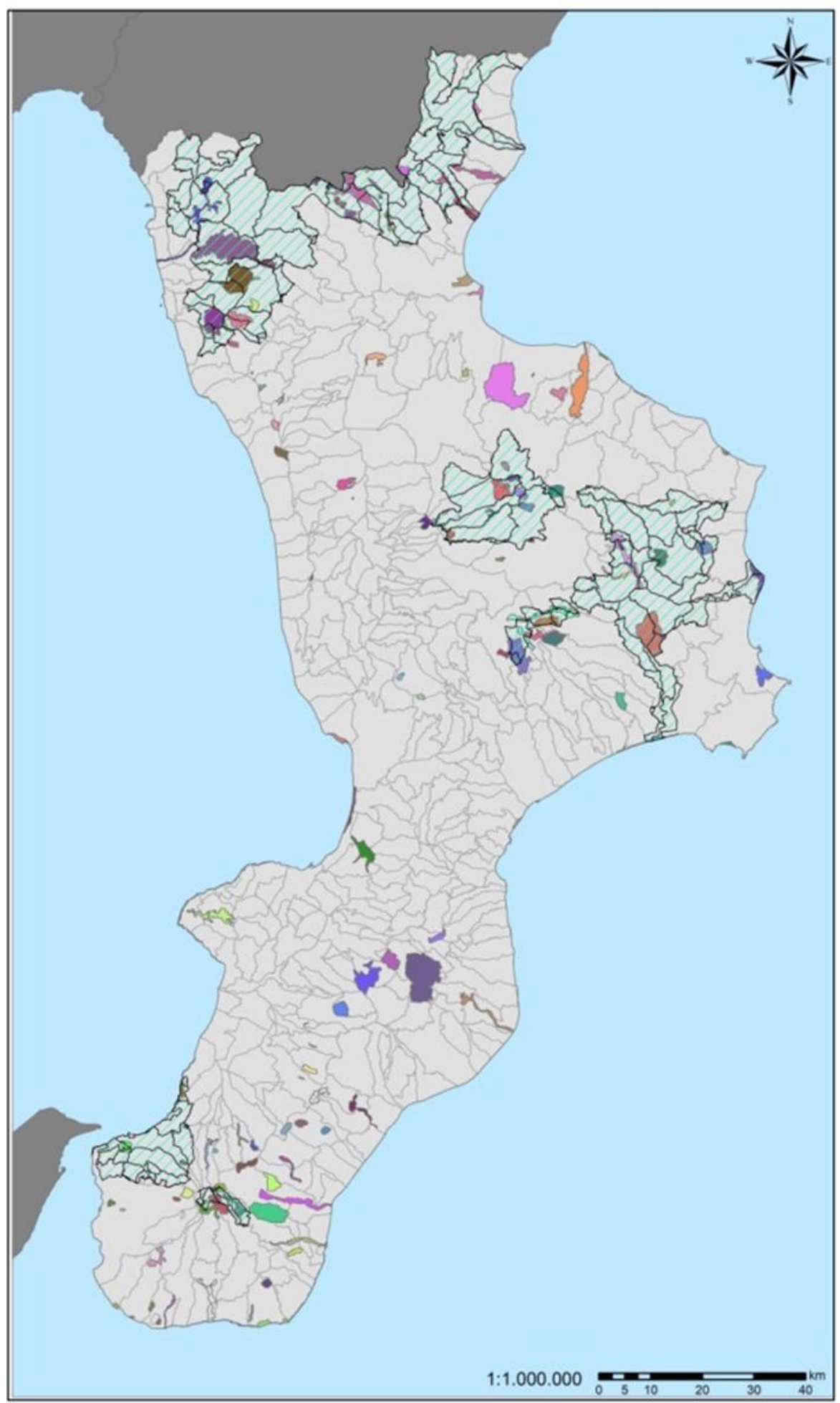

Fig. 1 SIC e le ZPS della Regione Calabria 
Legenda Fig. 1 - I SIC e le ZPS della Regione Calabria

Legenda

IT9210120, La Falconara, SIC

IT9210125, Timpa dell'Orso-Serra del Prete, SIC

IT9210130, Bosco di Chiaromonte-Piano lannace, SIC

IT9210146, Pozze di Serra Scorzillo, SIC

IT9210175, Valle Nera-Serra di Lagoforano, SIC

- IT9210265, Valle del Noce, ZSC

IT9310001, Timpone della Capanna, SIC

- IT9310002, Serra del Prete, SIC

IT9310003, Pollinello-Dolcedorme, SIC

IT9310004, Rupi del Monte Pollino, SIC

IT9310005, Cima del Monte Pollino, SIC

-IT9310006, Cima del Monte Dolcedorme, SIC

- IT9310007, Valle Piana-Valle Cupa, SIC

IT9310008, La Petrosa, SIC

IT9310009, Timpone di Porace, SIC

- IT9310010, Stagno di Timpone di Porace, SIC

$\square$ IT9310011, Pozze Boccatore/Bellizzi, SIC

$\square$ IT9310011, Pozze Boccatore/Bellizzi,

IT9310012, Timpa di S.Lorenzo, SIC
IT9310013, Serra delle Ciavole-Serra di Crispo, SIC

- IT9310014, Fagosa-Timpa dell'Orso, SIC

-IT9310015, II Lago (nella Fagosa), SIC

- IT9310017, Gole del Raganello, SIC

IT9310019, Monte Sparviere, SIC

- IT9310020, Fonte Cardillo, SIC

-IT9310021, Cozzo del Pellegrino, SIC

$\square$ IT9310022, Piano di Marco, SIC

IT9310023, Valle del Fiume Argentino, SIC

- IT9310025, Valle del Fiume Lao, SIC

IT9310027, Fiume Rosa, SIC

- IT9310028, Valle del Fiume Abatemarco, SIC

IT9310028, Valle del Fiume

IT9310030, Monte La Caccia, SIC

IT9310031, Valle del Fiume Esaro, SIC

- IT9310032, Serrapodolo, SIC

IT9310033, Fondali di Capo Tirone, SIC

$\square$ IT9310034, Isola di Dino, SIC

- IT9310035, Fondali Isola di Dino-Capo Scalea, SIC

$\square$ IT9310036, Fondali Isola di Cirella-Diamante, SIC

IT9310037, Isola di Cirella, SIC

- IT9310038, Scogliera dei Rizzi, SIC

- IT9310040, Montegiordano Marina, SIC

- IT9310041, Pinete di Montegiordano, SIC

IT9310042, Fiumara Saraceno, SIC

IT9310043, Fiumara Avena, SIC

IT9310044, Foce del Fiume Crati, SIC

IT9310044, Foce del Fiume Crati, SIC

IT9310045, Macchia della Bura, SIC

$\square$ IT9310047, Fiumara Trionto, SIC
$\square$ IT9310049, Farnito di Corigliano Calabro, SIC

IT9310051, Dune di Camigliano, SIC

- IT9310052, Casoni di Sibari, SIC

$\square$ IT9310054, Torrente Celati, SIC

$\square$ IT9310055, Lago di Tarsia, SIC

- IT9310056, Bosco di Mavigliano, SIC

$\square$ IT9310057, Orto Botanico - Università de

IT9310058, Pantano della Giumenta, SIC

IIT9310059, Crello, SIC

- IT9310060, Laghi di Fagnano, SIC

IT9310061, Laghicello, SIC

-IT9310062, Monte Caloria, SIC

IT9310063, Foresta di Cinquemiglia, SIC

IT9310064, Monte Cocuzzo, SIC

$\square$ IT9310065, Foresta di Serra Nicolino-Piano d'Albero, SIC

-IT9310066, Varconcello di Mongrassano, SIC

IT9310067, Foreste Rossanesi, SIC

-IT9310068, Vallone S. Elia, SIC

IT9310070, Bosco di Gallopane, ZSC

- IT9310071, Vallone Freddo, ZSC

IT9310072, Palude del Lago Ariamacina, ZSC

IT9310073, Macchia Sacra, ZSC

IT9310074, Timpone della Carcara, ZSC

IT9310075, Monte Curcio, ZSC

$\square$ IT9310076, Pineta di Camigliatello, ZSC

IT9310076, Pineta di Camigliatello,

$\square$ IT9310077, Acqua di Faggio, ZSC
IT9310079, Cozzo del Principe, ZSC

IT9310079, Cozzo del Principe, ZSC

IT9310081, Arnocampo, ZSC

IT9310082, S. Salvatore, ZSC

IT9310083, Pineta del Cupone, ZSC

- IT9310084, Pianori di Macchialonga, ZSC

- IT9310085, Serra Stella, ZSC

-IT9310126, Juri Vetere Soprano, ZSC

IT9310127, Nocelleto, ZSC

IT9310130, Carlomagno, ZSC

- T9320046, Starlomagno, ZSC

- IT9320046, Stagni solto

ITT320050, Pescaldo, SIC

IT9320097, Fondali da Crotone a Le Castella, SIC

IT9320100, Dune di Marinella, SIC
IT9320101, Capo Colonne, SIC

IT9320102, Dune di Sovereto, SIC

IT9320103, Capo Rizzuto, SIC

- IT9320104, Colline di Crotone, SIC

$\square$ IT9320106, Steccato di Cutro e Costa del Turchese, SIC

- IT9320110, Monte Fuscaldo, SIC

- IT9320111, Timpa di Cassiano- Belvedere, SIC

IT9320112, Murgie di Strongoli, SIC

- IT9320115, Monte Femminamorta, ZSC

$\square$ IT9320122, Fiume Lese, SIC

$\square$ IT9320123, Fiume Lepre, SIC

$\square$ IT9320129, Fiume Tacina, ZSC

IT9320129, Fiume Tacina, ZSC
IT9320185, Fondali di Staletti, SIC

IT9330087, Lago La Vota, SIC

IT9330087, Lago La Vota, SIC

IT9330088, Palude di Imbutillo, SIC

IT9330089, Dune dell'Angitola, SIC

- IT9330105, Foce del Crocchio - Cropani, SIC

IT9330107, Dune di Isca, SIC

- IT9330108, Dune di Guardavalle, SIC

IT9330109, Madama Lucrezia, SIC

$\square$ IT9330113, Boschi di Decollatura, SIC

IT9330114, Monte Gariglione, ZSC

- IT9330116, Colle Poverella, ZSC

IT9330117, Pinete del Roncino, ZSC

$\square$ IT9330124, Monte Contrò, SIC
$\square$ IT

$\square$ IT9330124, Monte Contrò, SIC

IT9330125, Torrente Soleo, ZSC
$\square$ IT9330128, Colle del Telegrafo, ZSC

- IT9330184, Scogliera di Staletti, SIC

- IT9340086, Lago dell'Angitola, SIC

$\square$ IT9340090, Fiumara di Brattirò (Valle Ruffa), SIC

- IT9340091, Zona costiera fra Briatico e Nicotera, SIC

IT9340092, Fondali di Pizzo Calabro, SIC

$\square$ IT9340093, Fondali di Capo Vaticano, SIC

$\square$ IT9340094, Fondali Capo Cozzo - S. Irene, SIC

- IT9340118, Bosco Santa Maria, SIC

IT9340119, Marchesale, SIC

IT9340120, Lacina, SIC

IT9350121, Bosco di Stilo - Bosco Archiforo, SIC

IT9350131, Pentidattilo, SIC

IT9350131, Pentidattilo, SIC

- IT9350132, Fiumara di Melito, SIC

$\square$ IT9350133, Monte Basilicò -Torrente Listi, SIC

IT9350135, Vallata del Novito e Monte Mutolo, SIC

IT9350136, Vallata dello Stilaro, SIC

IT9350137, Prateria, SIC

IT9350138, Calanchi di Maro Simone, SIC

- IT9350139, Collina di Pentimele, SIC

$\square$ IT9350140, Capo dell'Armi, SIC

IT9350141, Capo S. Giovanni, SIC

IIT9350142 Capo Spartivento, SIC

IT9350143, Saline Joniche, SIC

IT9350144, Calinchici Palzz

di Palizzi Marina, SIC

IT9350146, Fiumara Buonamico, SIC

- IT9350147, Fiumara Laverde, SIC

$\square$ IT9350148, Fiumara di Palizzi, SIC

IT9350149, Sant'Andrea, SIC

IT9350150, Contrada Gornelle, SIC

IT9350151, Pantano Flumentari, SIC

- IT9350152, Piani di Zervò, SIC

IT9350153, Monte Fistocchio e Monte Scorda, SIC

IT9350154, Torrente Menta, SIC

- IT9350155, Montalto, SIC

IT9350156, Vallone Cerasella SIC

IT9350157, Torrente Ferrain, SIC

IT9350158, Costa Viola e Monte S. Elia, SIC

$\square$ IT9350159, Bosco di Rudina, SIC

$\square$ IT9350160, Spiaggia di Brancaleone, SIC

- IT9350161, Torrente Lago, SIC

IT9350162, Torrente S. Giuseppe, SIC

$\square$ IT9350163, Pietra Cappa - Pietra Lunga - Pietra Castello, SIC

$\square$ IT9350164, Torrente Vasi, SIC

$\square$ IT9350165, Torrente Portello, SIC

$\square$ IT9350166, Vallone Fusolano (Cinquefrondi), SIC

IT9350167, Valle Moio (Delianova), SIC

- IT9350168, Fosso Cavaliere (Cittanova), SIC

IT9350169, Contrada Fossia (Maropati), SIC

$\square$ IT9350170, Scala-Lemmeni, SIC

- IT9350171, Spiagia di Pilati, SIC

IT9350172, Fondali da Punta Pezzo a Capo dell'Armi, SIC

IT9350172, Fondali da Punta Pezzo
IT9350173, Fondali di Scilla, SIC

IT9350173, Fondali di Scilla, SIC

IT9350174, Monte Tre Pizzi, SIC
IT9350175, Piano Abbruschiato, SIC

$\square$ IT9350175, Piano Abbruschiato, SIC
$\square$ IT9350176, Monte Campanaro, SIC

$\square$ IT9350177, Monte Scrisi, SIC

IT9350178, Serro d'Ustra e Fiumara Butrano, SIC

- IT9350179, Alica, SIC

- IT9350180, Contrada Scala, SIC

$\square$ IT9350181, Monte Embrisi e Monte Torrione, SIC

- IT9350182, Fiumara Careri, SIC

IT9350183, Spiaggia di Catona, SIC 
L'interpretazione del mosaico vegetazionale ha consentito di valutare per ogni habitat il Grado di Naturalità $(N)$ e lo Stato di Conservazione attraverso l'ILC (Index of Landscape Conservation) di Pizzolotto e Brandmayr (1996), utilizzando protocolli consolidati in letteratura (Ferrari et al., 2008).

La Naturalità dell'habitat è stata valutata utilizzando un indice in grado di sottolineare la posizione della comunità rilevata all'interno della serie dinamica di pertinenza rispetto la distanza dal climax (Jalas, 1955; Edarra, 1997; Meaza et al., 2000; Micciardi \& Gargini, 2003; Biondi \& Colosi, 2005; Ferrari et al., 2008; Spampinato et al., 2008; Biondi \& Nanni, 2005; Batzella et al., 2012).

Gli habitat sono stati classificati con una scala ordinale a sei gradi di Naturalità:

$0=$ Naturalità assente. Zone costruite e centri urbani;

1 = Naturalità molto bassa . Fitocenosi sinantropiche prive di naturalità (coltivi, impianti di specie esotiche, aree e insediamenti sparsi, ecc.);

2 = Naturalità bassa . Aree a scarsa naturalità (incolti, primi stadi della colonizzazione di colture abbandonate, rimboschimenti con specie autoctone, ecc.);

3 = Naturalità media . Fitocenosi semi-naturali. Stadi di ricostituzione della vegetazione forestale o conseguenza di una sua regressione (macchia e gariga secondaria, praterie steppiche, ecc.);

4 = Naturalità alta. Fitocenosi subnaturali vicino agli stadi di climax da cui differiscono principalmente nella composizione strutturale piuttosto che nella composizione floristica;

$5=$ Naturalità molto alta . Fitocenosi nello stadio di climax o molto vicino a quella fase.

Per stimare la Qualità e Vulnerabilità Ambientale la Carta della Natura (ISPRA, 2009) strumento previsto dalla legge quadro sulle Aree Protette (Legge 394/91), sarebbe stata la soluzione. La Carta Natura è uno strumento necessario per definire "le linee fondamentali dell'assetto del territorio con riferimento ai valori naturali ed ambientali" ed "individua lo stato dell'ambiente naturale in Italia, evidenziando $i$ valori naturali ed i profili di vulnerabilità territoriale" (Art. 3, comma 2-3, Legge 6.12.1991 n. 394). La Carta degli Habitat, carta base del sistema Carta della Natura, adotta il sistema classificatorio CORINE Biotopes (European Commission, 1991), basato sulle fitocenosi, integrato da informazioni di tipo litologico, geomorfologico, biogeografico e di uso del suolo. La nomenclatura CORINE Biotopes rende la Carta strategica nella difesa e conservazione dell'ambiente naturale e consente di stimare la Qualità e la Vulnerabilità di un Territorio, attraverso un set di Carte che, muovendo dalla Carta degli Habitat, comprendere la Carta del Valore Ecologico, la Carta della Sensibilità Ecologica, la Carta della Pressione Antropica e la Carta della Fragilità Ambientale.

Realizzata di norma in sinergia fra l'Istituto Superiore per la Protezione e la Ricerca Ambientale (ISPRA ex ANPA e APAT) e l'Agenzia della Protezione Ambientale (ARPA) della regione interessata, è strutturata in modo gerarchico, per produrre carte derivate di minore e/o maggiore dettaglio. Ad oggi su 20 regioni 13 ne sono dotati: Abruzzo, Basilicata, Campania, Friuli Venezia Giulia, Lazio, Liguria, Molise, Puglia, Sardegna, Sicilia, Umbria, Valle d'Aosta. 
Non disponendo la regione Calabria della Carta della Natura in scala 1:50.000, ma in compenso dotata della Carta delle Aree Sensibili alla Desertificazione (Barbera et al., 2005) gli AA., per stimare la Sensibilità Ambientale nei Siti Natura 2000 che insistono sui corridoi ecologici del versante jonico calabrese, hanno, quindi, adottato la metodologia MEDALUS, messa a punto nell'ambito del Progetto Europeo di Ricerca MEditerranean Desertification And Land Use (Kosmas et al., 1999). Hanno stimato il grado di Sensibilità Ambientale (Fig. 2) muovendo dal computo della Qualità del Clima (CQI), della Qualità della Vegetazione (VQI), della Qualità del Suolo (SQI) e della Qualità della Gestione del Territorio (MQI). Il livello di sensibilità, noti i quattro indici di qualità, è espresso dalla seguente formula:

$$
E S A=(S Q I * C Q I * V Q I * M Q I) 1 / 4
$$

L'indice individua le aree con crescente sensibilità ambientale, secondo la seguente classificazione in cui sono evidenziati i differenti valori che tale indice può assumere:

- Aree (ESAs) non minacciate: aree non soggette e non sensibili;

- Aree (ESAs) potenziali: minacciate dalla desertificazione, ossia soggette ad un significativo cambiamento climatico. Si tratta per lo più di aree marginali abbandonate, non gestite in modo appropriato;

- Aree (ESAs) fragili: dove qualsiasi cambiamento del delicato equilibrio dei fattori naturali o delle attività umane, molto probabilmente, porterà alla desertificazione;

- Aree (ESAs) critiche: altamente degradate a causa dell'inadeguato uso del terreno che presenta una minaccia all'ambiente delle aree circostanti.

Per quanto attiene il Clima i dati pluviometrici e termometrici sono tratti dagli Annali Idrologici del Servizio Idrologico. La serie storica adottata è il trentennio 1961-90. La scelta del periodo è imposta dalla comunità scientifica internazionale ai fini della confrontabilità dei risultati. Per la Vegetazione è stata utilizzato il geodatabase CORINE Land Cover del $2000\left(3^{\circ}\right.$ e $4^{\circ}$ livello) e per la Gestione del Territorio, oltre al $C L C$, il $6^{\circ}$ Censimento Generale dell'Agricoltura (ISTAT, 2013). Per il Suolo è stata digitalizzata la Carta dei Suoli dell'ARSSA (2003).

L'ArcView (GIS) 10.1 è il software utilizzato per l'acquisizione e la gestione dell'informazione territoriale.

Per le finalità dello studio non era necessario rivisitare i 179 SIC bensì un campione rappresentativo. La scelta è caduta sui fiumi in quanto corridoi ecologici; nello specifico, sono stati caratterizzati i 10 SIC che insistono sul versante ionico della regione, specificatamente le foci. 


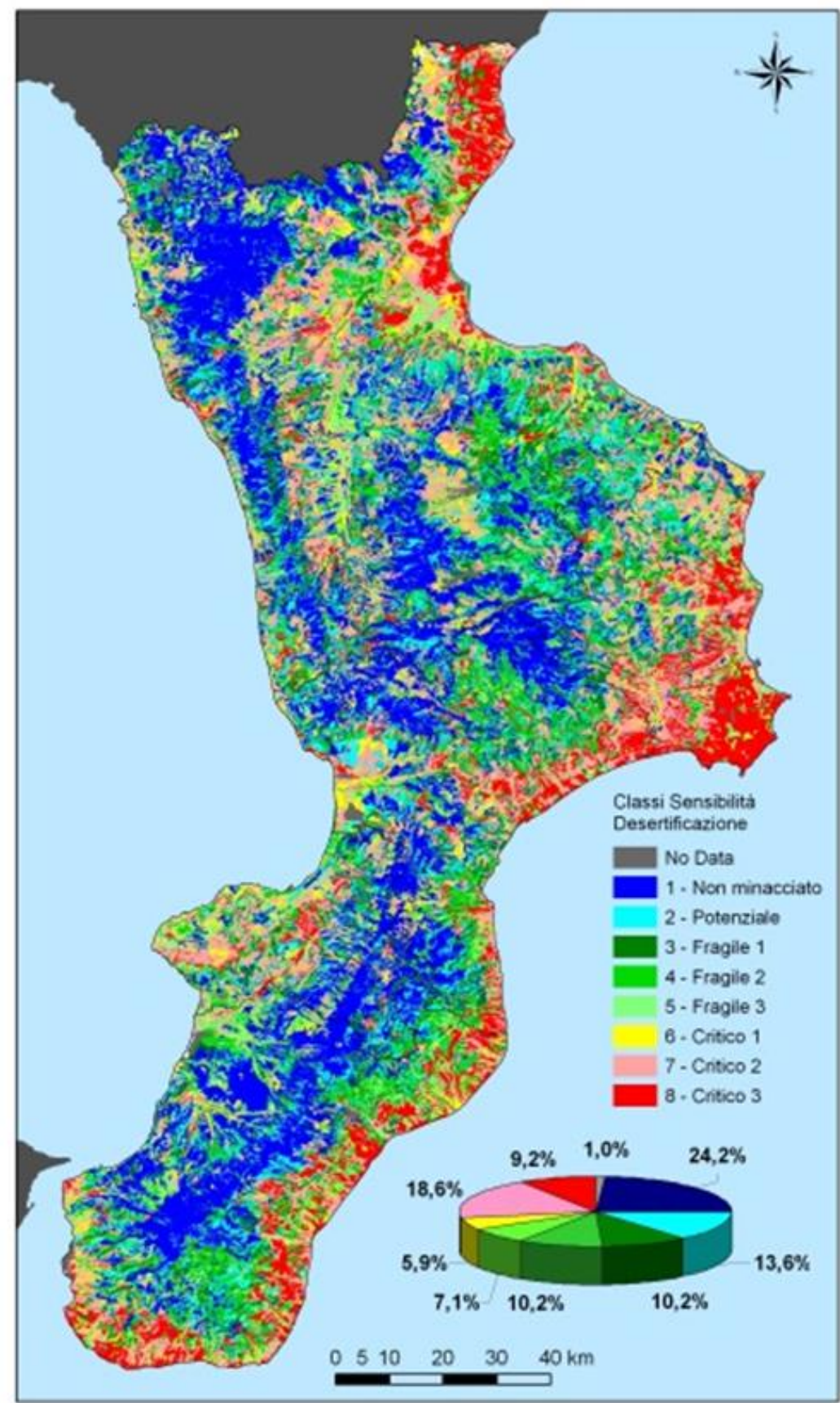

Fig. 2 Carta della Vulnerabilità Ambientale Regione Calabria (sensu Medalus) 


\section{Risultati}

Lo studio è consistito, preliminarmente, nel verificare la Naturalità/Artificialità degli stessi muovendo dalla Carta dell'Uso del Suolo CLC IV livello del 2012 (Fig. 3) tradotta e interpretata (Tab. 1) a scala di dettaglio per i 10 SIC (in Allegato I sono riportate le 10 Carte dell'Uso del Suolo secondo CLC IV livello 2012).

Per la stima della Naturalità/Artificialità è stata adottata la legenda riportata in Tab. 2. Per quanto concerne i risultati di questa prima fase, emerge uno scenario variegato (Fig. 4 e Tab. 2):

- il SIC Foce del Crocchio Cropani non ha traccia di naturalità, prevale l'ambiente seminaturale, che incide per il $62 \%$, e il rimanente $38 \%$ è territorio antropizzato;

- i SIC delle Foci dei Fiumi Crati, Neto e della Fiumara Avena presentano modestissime incidenze territoriali di naturalità, rispettivamente, $3 \%, 7 \%$ e $17 \%$;

- i SIC del Fiume Saraceno e della Fiumara Trionto fanno rilevare dei buoni valori di naturalità, nello specifico $37 \%$ e $36 \%$;

- il SIC Fiumara di Melito ha la migliore performance di naturalità di territorio (69\%), seguito dai SIC delle Fiumare Laverde (64\%) e Buonamico (57\%).

Per rendere più agevole la lettura dei risultati si è ritenuto opportuno integrare la rappresentazione di Fig.4 con una più sintetica e intuitiva, articolata in tre classi (Fig.5):

- artificiale (riunendo le classi le subclassi 1 e 2);

- seminaturale (classe 3);

- naturale (classe 4 e 5).

Emerge che:

- i SIC delle Fiumare Avena e Trionto presentano un'artificialità di territorio superiore al $50 \%$;

- il SIC della Fiumara Amendolea un'artificialità di poco inferiore al 50\%;

- i SIC del Fiume Crati e della Foce del Crocchio Cropani si attestano a quota $60 \%$ di semi-naturalità;

- il SIC del Foce Neto registra una seminaturalità del 50\%;

- $\quad$ i SIC delle Fiumare Melito, Bonamico e Laverde sono dotati di un'ottima percentuale di naturalità del territorio.

In Allegato II sono riportate le 10 Carte della Naturalità/Artificialità derivate dalle rispettive 10 Carte dell'Uso del Suolo secondo CLC IV livello 2012.

Riguardo l'analisi della Vulnerabilità Ambientale, muovendo dall'interrogazione della Banca Dati Georiferita del Rischio Desertificazione della Regione Calabria (Barbera et al., 2005), di cui alla Tab.3 e alla Fig. 6, emerge uno scenario ancora più complesso poiché il modello adotta una classificazione a 5 classi, di cui due distinte in tre subclassi.

Interessante in alcuni casi la complementarietà del dato Sensibilità Ambientale rispetto alla Naturalitàl Artificialità (in Allegato III sono riportate le 10 Carte della Sensibilità Ambientale sensu MEDALUS).

Ad esempio, il SIC della Fiumara di Melito (Fig. 6) che, esprime la maggiore naturalità fra i 10 $S I C$, risulta ai fini della Sensibilità Ambientale il più critico. Anche in questo caso le classi sono 
state riunite in tre categorie di territori (Fig. 7):

- poco sensibili

- fragili

- critici.

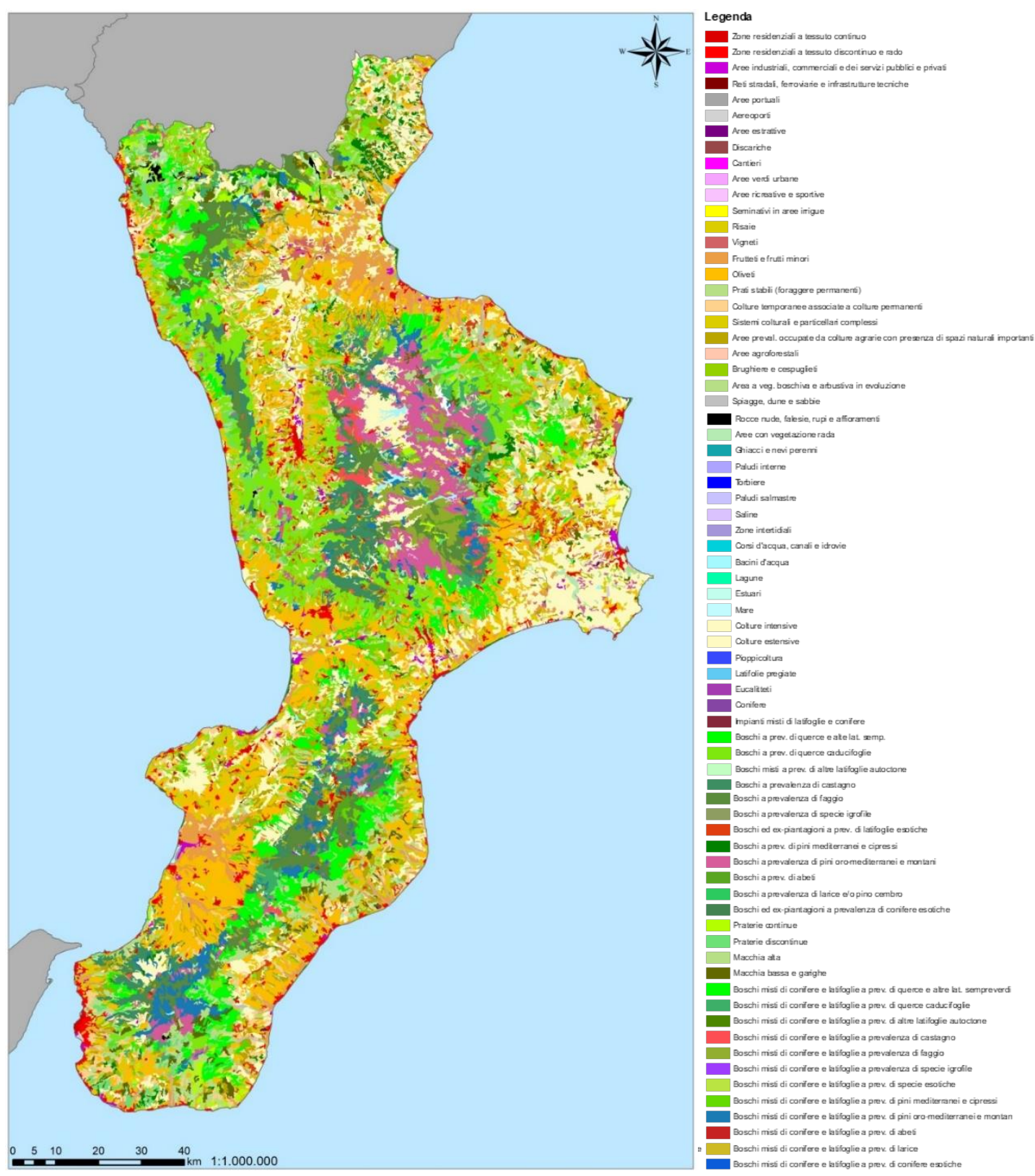

Fig. 3 Carta dell’Uso del Suolo CLC 2012 - IV livello, Regione Calabria 


\begin{tabular}{|c|c|c|c|c|c|c|c|c|c|c|c|c|c|c|c|}
\hline 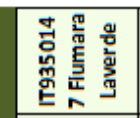 & : & & F. & . & $\underset{\sim}{\approx}$ & ô & : & & & & & & $\frac{m}{\infty}$ & & $\frac{0}{7}$ \\
\hline 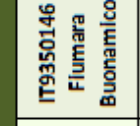 & & & $\mid \begin{array}{l}\pi \\
\vdots \\
=\end{array}$. & - $\cdot$. & $\overrightarrow{\mathfrak{w}_{2}} \tilde{z}$ & & 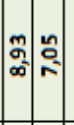 & & & & : & $\Xi_{0}^{\infty}$ & 总 & & 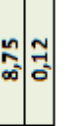 \\
\hline 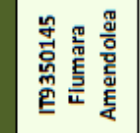 & 売 & . & & 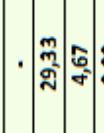 & $\vec{n}$ & مُ & $\mid$ & & & & : & 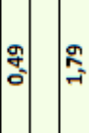 & $\frac{\Phi}{\sigma}$ & & 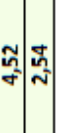 \\
\hline 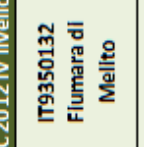 & న్ & ${ }^{\circ}$ & . & | $\cdot \mid \begin{array}{l}\infty \\
\vdots \\
\alpha\end{array}$ & $\cdot||_{\alpha}^{7}$ & . & $\cdot$ & 总 & 总 & $\cdot$ & . & $\cdot|\cdot| \tilde{N}_{0}^{2}$ & . & 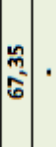 & $\mid$ \\
\hline 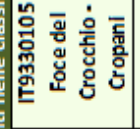 & & & $\mid \begin{array}{c}\mid \\
0\end{array}$ & $\left|\begin{array}{l}0 \\
0 \\
=1\end{array}\right|$ & . & $\cdot$ & $\cdot \cdot \cdot$ & $\cdot$ & 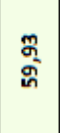 & ${ }^{\prime}$ & . & & & & $\cdot\left|\begin{array}{l}2 \\
m \\
m\end{array}\right|$ \\
\hline 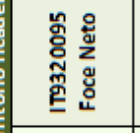 & & & 营 & $\left|\begin{array}{l}\mid \\
-i\end{array}\right|$. & . & . &. & $\cdot$ & 怘 & $\cdot$ & ${ }^{\circ}$ & & & â & $\left|\begin{array}{c}m \\
-=1\end{array}\right|$ \\
\hline 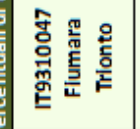 & $\underset{\sim}{\mathbb{N}}$ & & 范 & 商: & & 旾 & 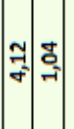 & 导 & & & & $\mid \begin{array}{l}f \\
0 \\
0\end{array}$ & & & $\cdot \mid$ \\
\hline 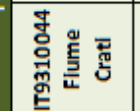 & . & ֻٕ & శ్ & 苔: & . & . & . & . & 商 & $\cdot$ & . & & & 怘 & $\cdot \vec{\partial}$ \\
\hline 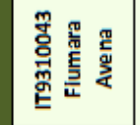 & . & & 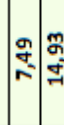 & ్ㅜㅇ & 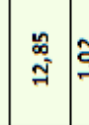 & 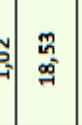 & & . & 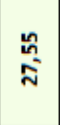 & & . & & & $\mid$ & $\because$ \\
\hline 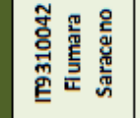 & 兽 & & 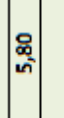 & $\mid$ & 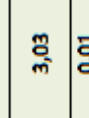 & 空 & $\stackrel{0}{\circ}$ & & 总 & 参 & & : & & 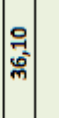 & : \\
\hline 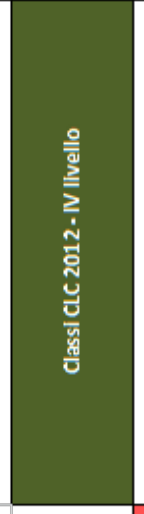 & 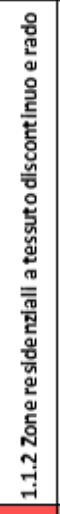 & & 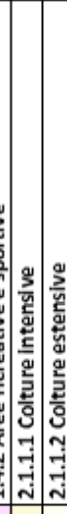 & 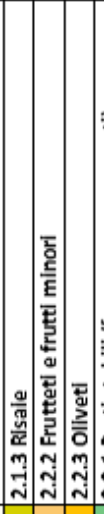 & 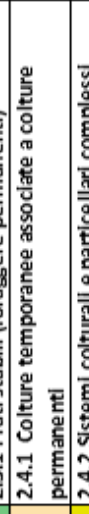 & 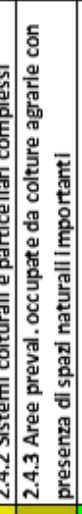 & 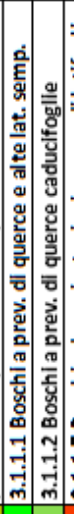 & 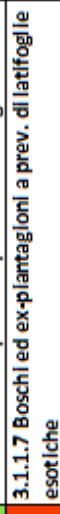 & 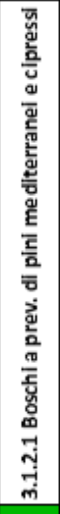 & 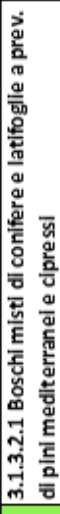 & 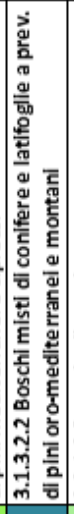 & 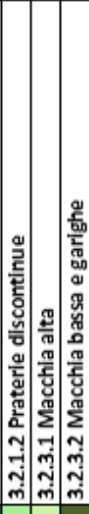 & 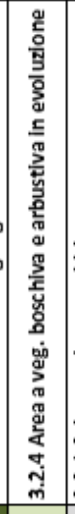 & 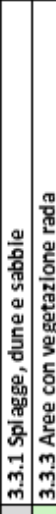 & 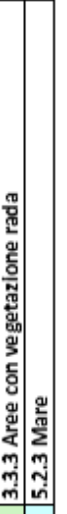 \\
\hline
\end{tabular}




\begin{tabular}{|c|c|}
\hline Naturalità & Classi CLC IV liv. 2012 \\
\hline $\mathbf{0}$ & 1.2.3 Aree portuali \\
\hline 1 & $\begin{array}{l}\text { 1.1.2 Zone residenziali a tessuto discontinuo e rado } \\
\text { 1.4.2 Aree ricreative e sportive } \\
\text { 2.1.3 Risaie } \\
\text { 2.2.2 Frutteti e frutti minori } \\
\text { 2.2.3 Oliveti } \\
\text { 2.4.1 Colture temporanee associate a colture permanenti } \\
\text { 2.4.2 Sistemi colturali e particellari complessi } \\
\text { 2.1.1.1 Colture intensive } \\
\text { 2.1.1.2 Colture estensive } \\
\text { 3.1.1.7 Boschi ed ex-piantagioni a prev. di latifoglie esotiche }\end{array}$ \\
\hline 2 & $\begin{array}{l}\text { 2.3.1 Prati stabili (foraggere permanenti) } \\
\text { 2.4.3 Aree preval. occupate da colture agrarie con presenza di spazi naturali importanti } \\
\text { 3.2.1.2 Praterie discontinue }\end{array}$ \\
\hline 3 & $\begin{array}{l}\text { 3.2.4 Area a veg. boschiva e arbustiva in evoluzione } \\
\text { 3.3.3 Aree con vegetazione rada } \\
\text { 3.1.2.1 Boschi a prev. di pini mediterranei e cipressi }\end{array}$ \\
\hline 4 & $\begin{array}{l}\text { 3.2.3.2 Macchia bassa e garighe } \\
\text { 3.1.3.2.1 Boschi misti di conifere e latifoglie a prev. di pini mediterranei e cipressi }\end{array}$ \\
\hline 5 & $\begin{array}{l}\text { 3.3.1 Spiagge, dune e sabbie } \\
\text { 3.1.1.1 Boschi a prev. di querce e alte lat. semp. } \\
\text { 3.1.1.2 Boschi a prev. di querce caducifoglie } \\
\text { 3.1.3.2.2 Boschi misti di conifere e latifoglie a prev. di pini oro-mediterranei e montani } \\
\text { 3.2.3.1 Macchia alta }\end{array}$ \\
\hline
\end{tabular}

Tab. 2 Classi di Naturalità/Artificialità rilevati nei 10 SIC

\section{Considerazioni}

L'istituzione di un SIC dovrebbe concretamente contribuire a:

- mantenerli integri;

- promuovere, laddove necessario, opere di deframmentazione territoriale, ovvero di rimozione o di mitigazione delle barriere e delle strozzature che penalizzano le aree di connessione;

- perseguire il miglioramento della qualità degli habitat delle specie individuate come prioritarie, ovvero delle specie più importanti e vulnerabili in situazione di territorio frammentato, che mostrano problemi di distribuzione e dispersione;

- conservare e migliorare le connettività in quanto di importanza a livello comunitario;

- favorire l'adattamento della biodiversità ai cambiamenti climatici, problematica sempre più pressante che costringe molte specie a rapidi spostamenti alla ricerca di habitat idonei.

Per raggiungere i superiori obiettivi bisognerebbe caratterizzare un SIC in modo rigoroso e responsabile. Dallo studio emergono le seguenti considerazioni:

- la Rete Natura 2000 adotta un approccio moderno rispetto alle classiche Aree Protette. Consente una convivenza con le attività antropiche. A fronte di questa opportunità si paga un prezzo. Le perimetrazioni delle aree da tutelare (SIC e ZPS) vengono delimitate in modo approssimativo, mortificando la proprietà privata. N'è riprova questo studio che evidenzia, in modo inequivocabile, come in più casi la delimitazione nei SIC venga eseguita su territori che non hanno poco o nulla di Naturale e di Seminaturale. Entrando nel merito su 10 SIC 3 presentano un'estensione territoriale con Naturalità inferiore al 10\%, 1 meno del 20\% e 3 del $40 \%$; solo tre superano il 50\%. Di contra, le estensioni di territori antropici (artificiali) sono rilevanti: 2 SIC più del $50 \%$ di territorio, 1 con poco meno del $50 \%, 2$ con poco più di un terzo.

Entrando nel merito del Seminaturale, area di frontiera ecotonica fra il Naturale e l'Artificiale, non attribuibile quindi a nessuna delle due classi, emerge uno scenario sconfortante. In definitiva sono da promuovere in termini di Qualità Ambientale 3 su 10 SIC. 


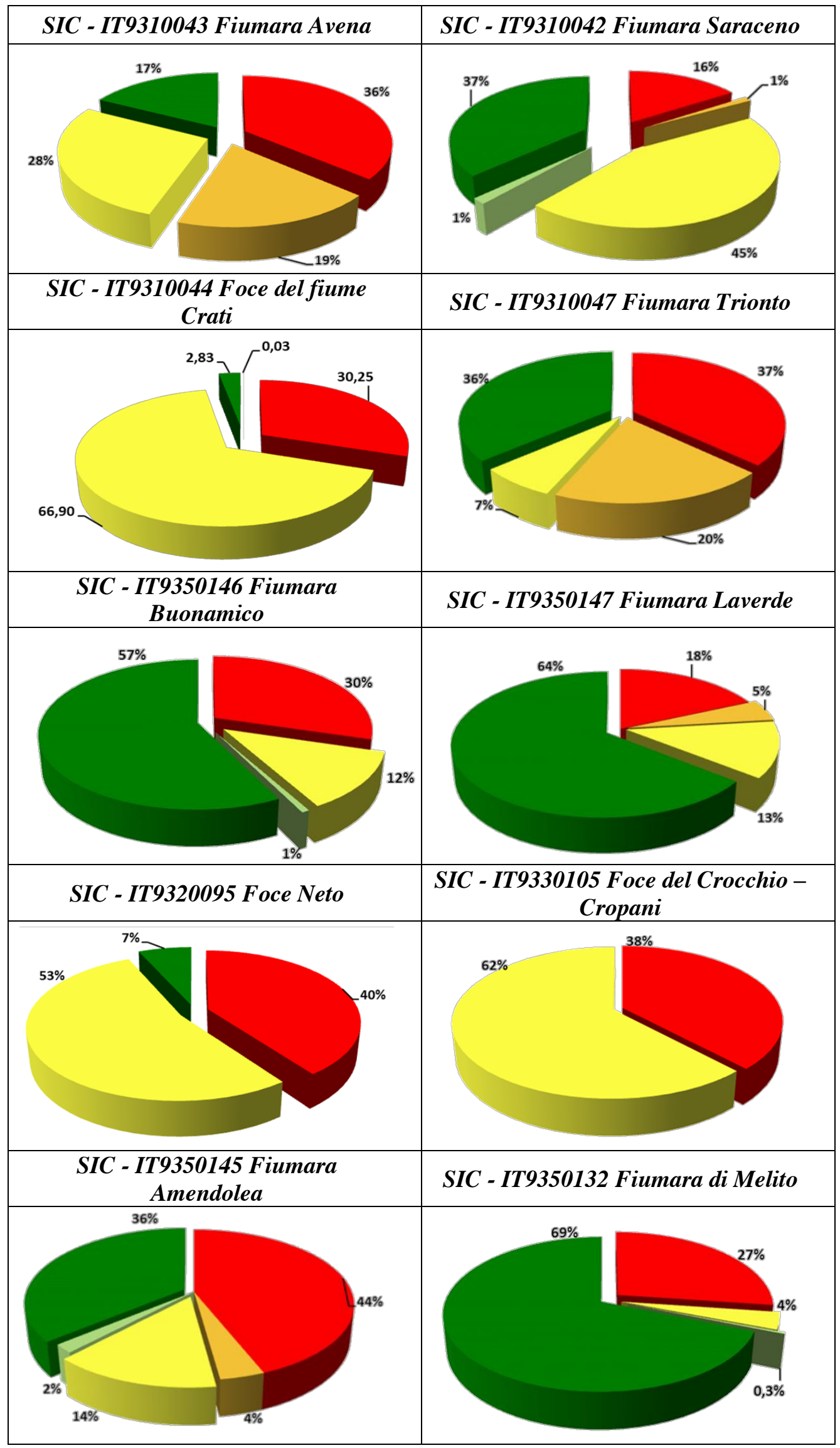

Fig. 4 - Classi (in \%) di naturalità/artificialità (6 classi) dei 10 SIC 

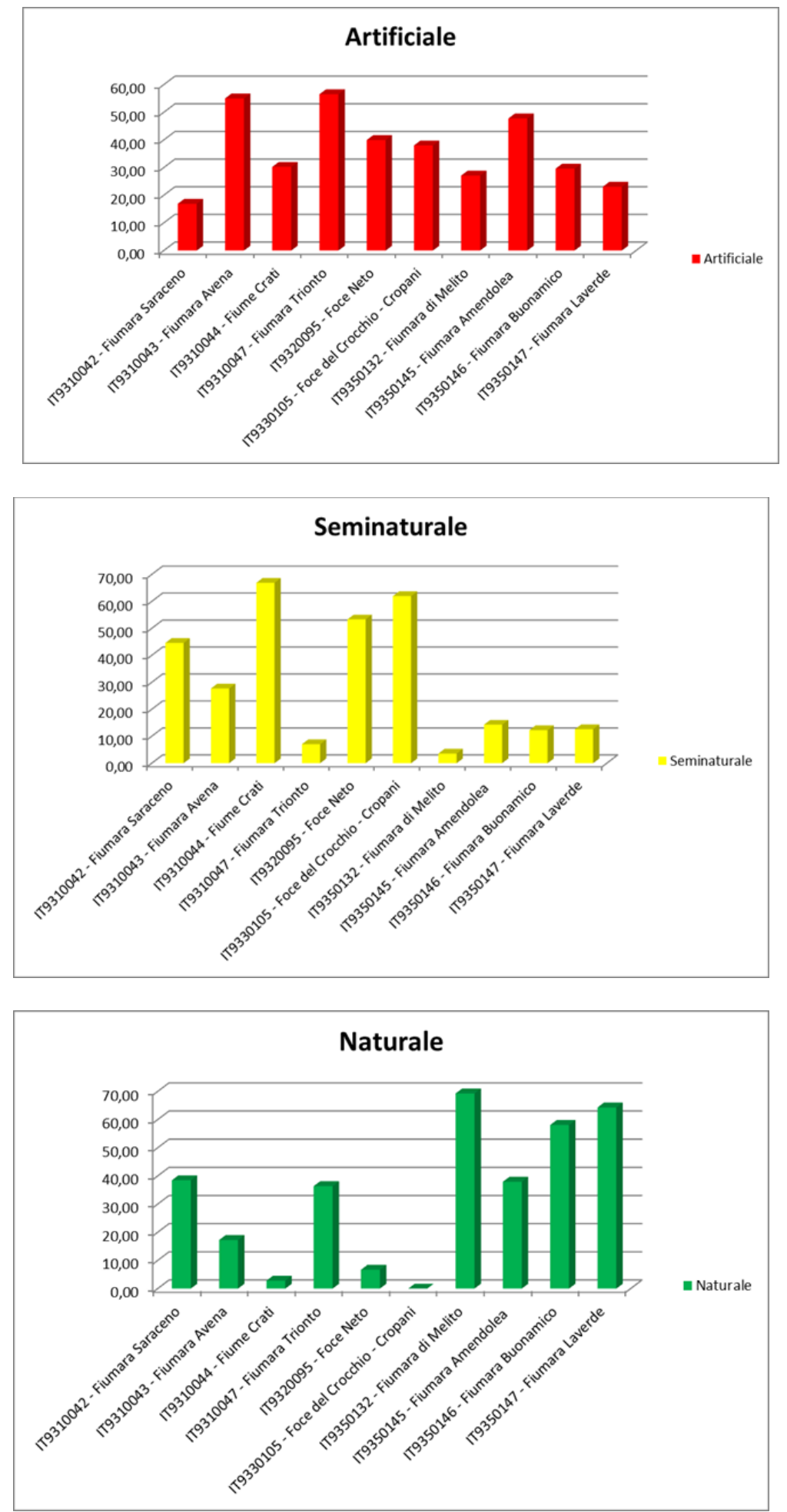

Fig. 5 Classi (in \%) di naturalità/artificialità dei 10 SIC 


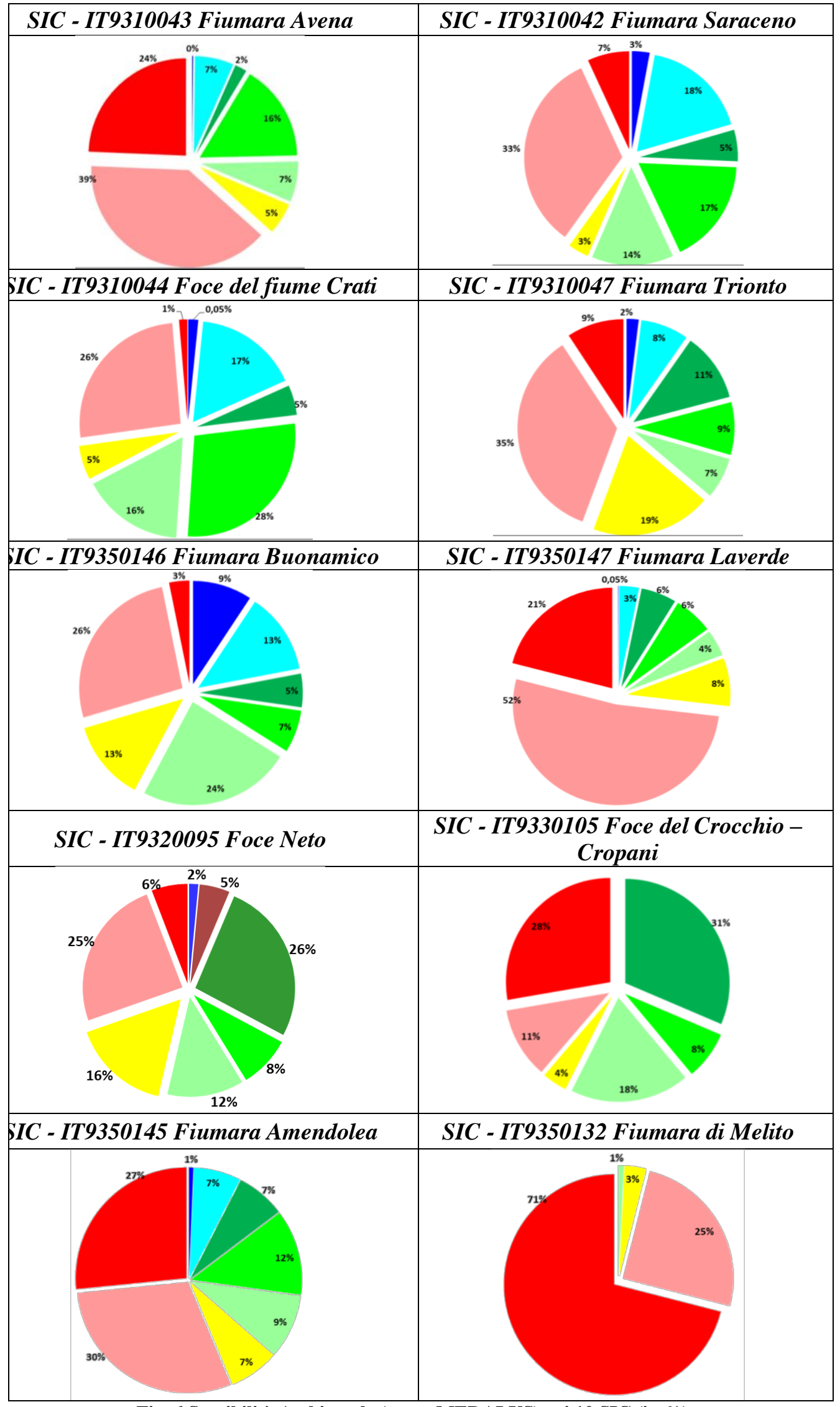

Fig. 6 Sensibilità Ambientale (sensu MEDALUS) nei 10 SIC (in \%) 

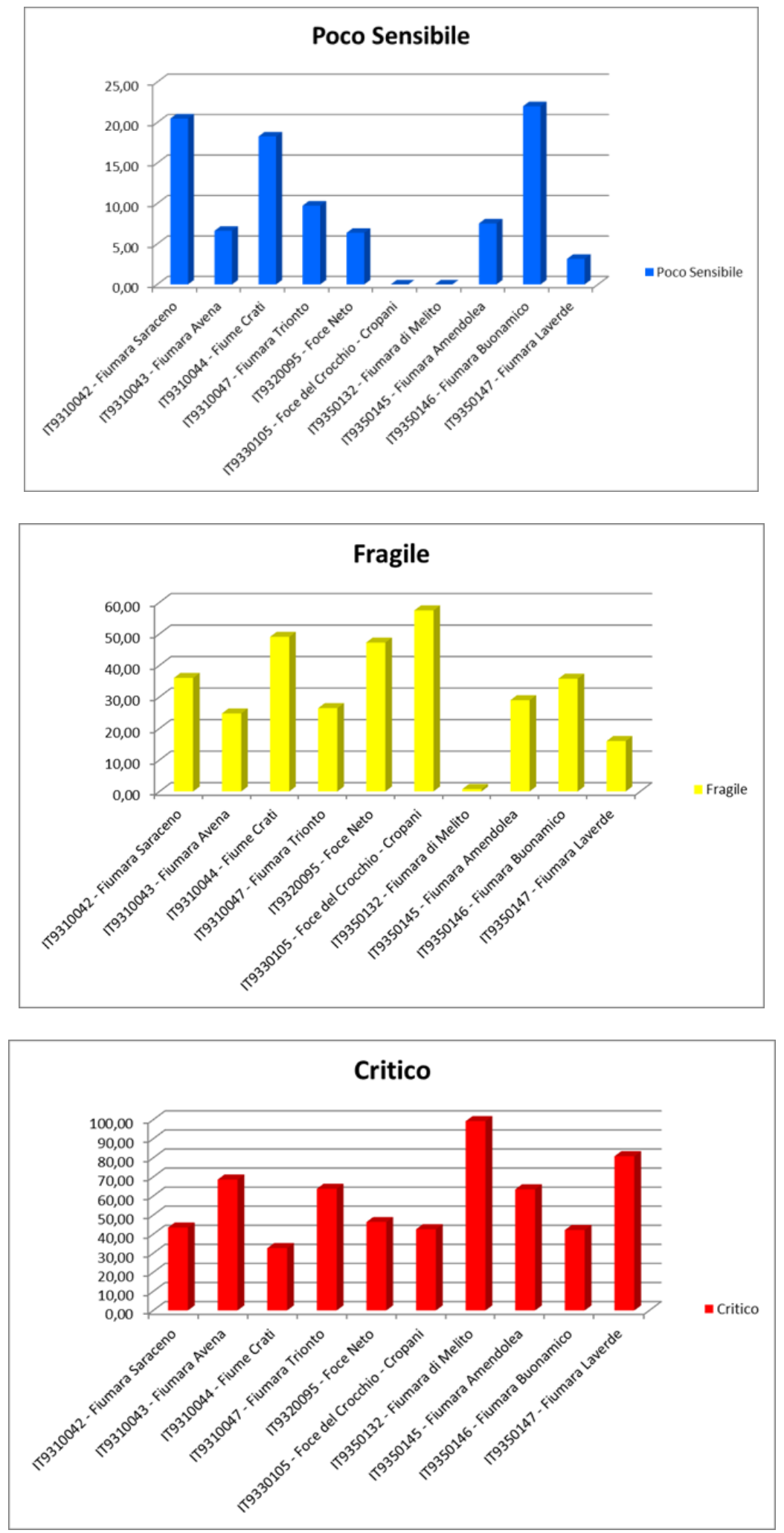

Fig. 7 Sensibilità Ambientale (3 classi) nei 10 SIC (in \%) 
- Il sistema di classificazione da adottare nella caratterizzazione di un SIC o ZPS dovrebbe essere solo e soltanto il CORINE Biotopes. Si basa sulle fitocenosi, ma utilizza anche informazioni di tipo litologico, geomorfologico, biogeografico e di uso del suolo. Ai livelli di maggiore dettaglio è determinante l'approccio fitosociologico, che lo rende adeguato alla corretta individuazione degli habitat alla scala di indagine. La sua struttura gerarchica permette di scegliere per ciascun tipo di habitat il livello coerente con il dettaglio adottato. La scelta della nomenclatura CORINE Biotopes per la scala 1:10.000, scala auspicata, renderà le carte geobotaniche istituzionali, in ragione della ufficialità in ambito Europeo di questo sistema classificatorio.

Muovendo dalla legenda CORINE Biotopes, realizzata per tutta Europa, è stata già eseguita una prima selezione dei tipi di habitat presenti in Italia e potenzialmente cartografabili alla scala 1:10.000. Gli habitat sono quelli presumibilmente presenti in Italia sulla base della letteratura geobotanica disponibile e dei rilevamenti di campo effettuati dal Servizio Carta della Natura del Dipartimento Difesa della Natura dell'ISPRA (ex-APAT).

In attesa che si consolidi quanto prima questo orientamento è opportuno operare riportando le eventuali corrispondenze tra $\mathrm{i}$ tipi di habitat selezionati ed i codici degli altri sistemi classificativi (Rete Natura 2000 ed EUNIS). Questi collegamenti tra diversi sistemi classificatori consentiranno di evidenziare quegli habitat presenti in Italia e non riscontrabili tra i codici europei di Rete Natura 2000.

Le corrispondenze tra i codici della Rete Natura 2000 della "Direttiva Habitat" e del CORINE Biotopes, salvo adeguamenti alla realtà italiana, sono desumibili dai documenti ufficiali della Comunità Europea per l'interpretazione degli habitat (Commission of the European Communities).

Le corrispondenze tra i codici CORINE Biotopes ed EUNIS sono basate sulle pubblicazioni certificate dalla Commissione Europea, sul manuale pubblicato dall'APAT sulla classificazione degli habitat EUNIS per la realtà italiana e sui data base dell'European Environment Agency (http://eunis.eea.europa.eu/habitats -code.jsp).

\section{Caso studio SIC Foce Neto}

\subsection{Sito}

L'area di studio conincide con l'estensione del SIC Foce Neto IT9320095 per complessivi 583 ettari. Il SIC è localizzato sulla costa Ionica calabrese, a nord del Comune di Crotone, nella medesima provincia (Tavoletta I.G.M.I. 238 IV SE sez. CB), e s'inserisce nella più ampia ZPS IT9320302 Marchesato e Foce Neto. Il Neto è il secondo fiume della Calabria per lunghezza e portata, nasce a circa $1700 \mathrm{~m}$ di quota, sugli alti monti della Sila e raggiunge dopo $80 \mathrm{~km}$ il mar Ionio, a circa metà distanza dai comuni di Crotone e di Strongoli Marina. Il SIC include ambienti umidi rappresentati dalla foce del Fiume Neto, da ripisilve, piccole aree palustri ed un tratto di fascia costiera ampia $2 \mathrm{~km}$. (Fig. 8 ).

Il SIC rientra nell'IBA n.149, insieme all'area Alto Marchesato e alla Foce del Fiume Tacina. E' un sito di interesse naturalistico e conservazionistico, poiché luogo di transito, sosta temporanea e nidificazione di molte specie di uccelli acquatici marini. Il SIC ospita un'avifauna stanziale e migratoria ed è sito di riproduzione delle tre specie di cheloni calabresi Caretta caretta, Emys orbicularis e Testudo hermanni.

Tra le specie vegetali, si segnala la presenza di taxa inseriti nella Lista Rossa Regionale e/o Nazionale fra i quali si citano Clematis viticella subsp. viticella, Ephedra distachya subsp. distachya, Euphorbia terracina, Iris foetidissima, Iris pseudacorus, Juniperus phoenicea s.1., 
Medicago marina, Pancratium maritimum, Quercus robur, Sarcopoterium spinosum, Thymelaea hirsuta, Anacamptis morio, Barlia robertiana, Ophrys bertolonii, Ophrys holosericea subsp. apulica, Orchis laxiflora, ecc.

Il paesaggio si presenta molto antropizzato, sono prevalenti aree agricole e agro-pastorali, aree di bonifica anche recenti e insediamenti di case sparse.

Dal punto di vista geologico, le formazioni sulle quali ricade il sito sono costituite da depositi dell'Olocene composti da alluvioni mobili, ciottolose e sabbiose dei letti fluviali e depositi del litorale ( $a c)$; da detriti di frana $(a f)$ e da dune e sabbie eoliche stabilizzate (dl), (Fig. 9).

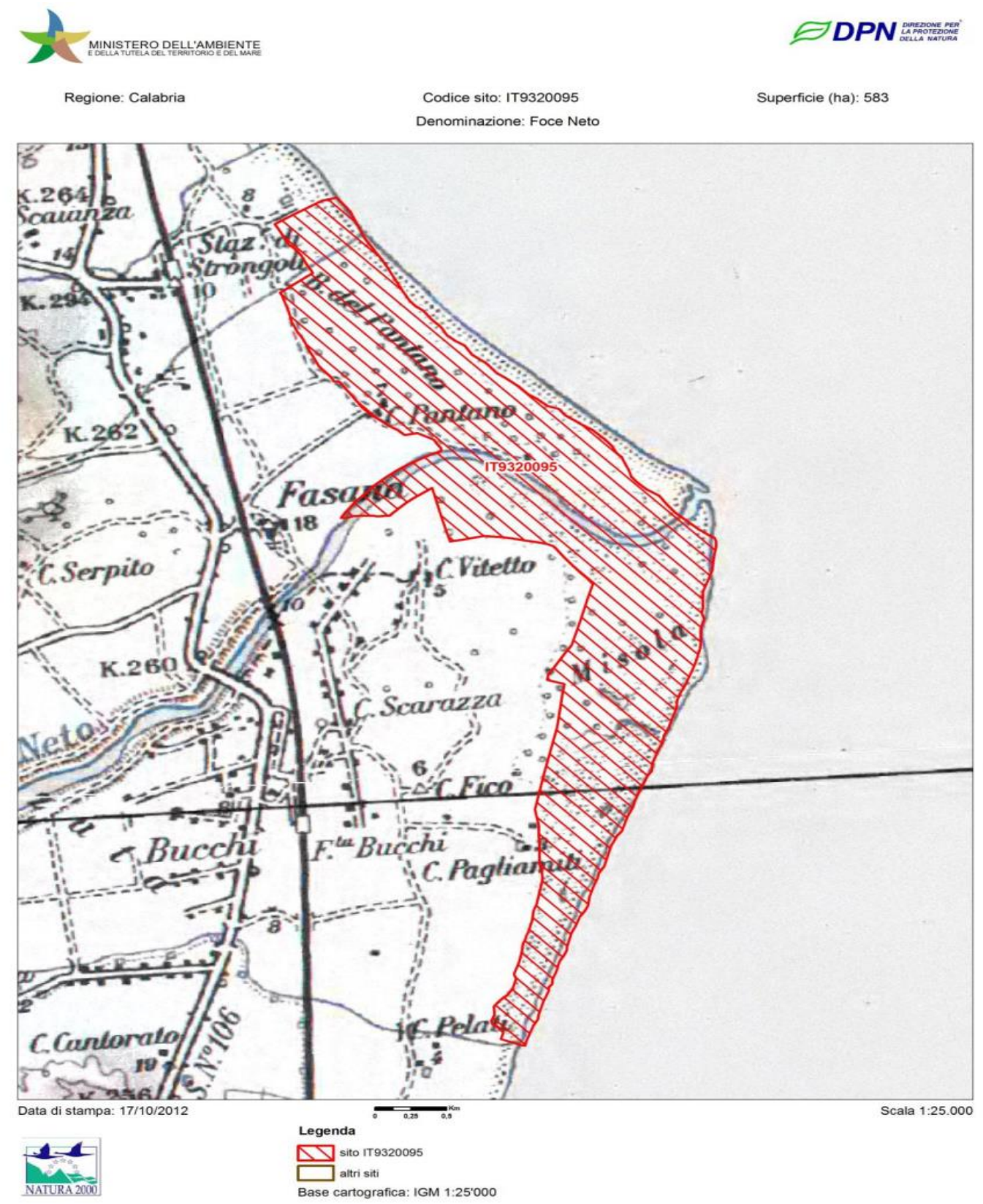

Fig. 8 Localizzazione del SIC Foce Neto IT9320095 

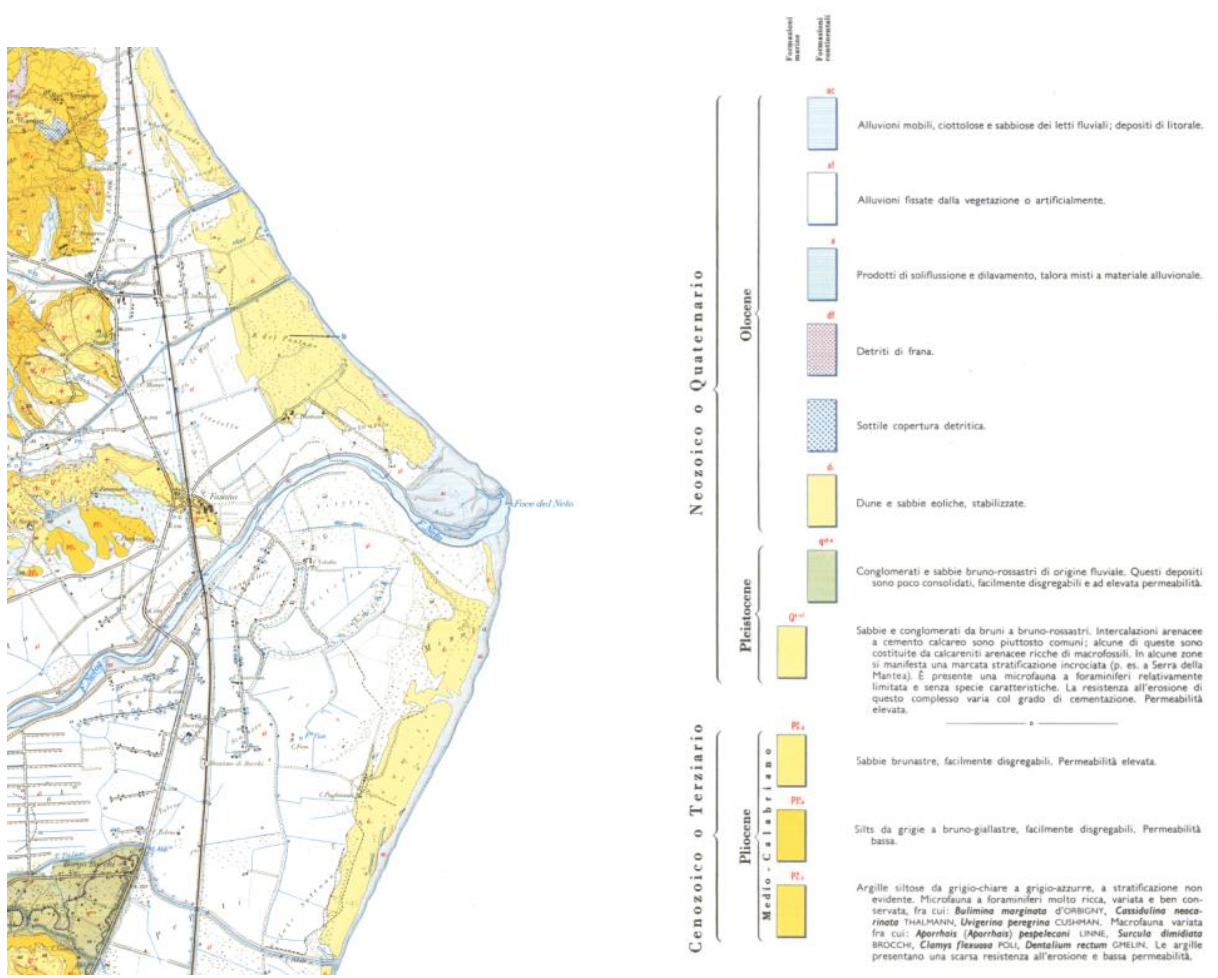

Fig. 9 Stralcio della Carta Geologica della Calabria, 1:25.000, Foglio 238 - IV S.E.

Il territorio si presenta pianeggiante e di natura alluvionale con suoli di tipo Typic Xerofluvents (Eutric Fluvisols) con profilo Ap-C e tessitura limoso-argillosa e reazione subalcalina. Nella parte retrostante la linea di costa, a limite con l'area di demanio costiero, il suolo è costituito da sabbie fini e quarzose di tipo embrionale ed è ascrivibile agli Xeropsamments.

Il clima è caratterizzato da una chiara impronta mediterranea. Per la caratterizzazione climatica sono stati utilizzati i dati relativi alla stazione termopluviometrica di Crotone, a circa $9 \mathrm{~km}$ dall'area oggetto di studio (Fig. 10). La temperatura media annua è di $17.6{ }^{\circ} \mathrm{C}$. Le precipitazioni sono scarse, non superando i $670 \mathrm{~mm}$ annui e sono concentrate durante i mesi invernali. Il Climogramma di Walter \& Lieth evidenzia una distribuzione delle precipitazioni e temperature propriamente di tipo mediterraneo, con un periodo secco della durata di circa quattro mesi. Le piogge si concentrano da Ottobre a Dicembre, con una media di $100 \mathrm{~mm}$ mensili. Le temperature medie raggiungono il massimo nel mese di Luglio $\left(27^{\circ} \mathrm{C}\right)$ e il minimo nel mese Gennaio $\left(11^{\circ} \mathrm{C}\right)$. La caratterizzazione bioclimatica fa riferimento a Rivas-Martínez \& Loidi Arregui (1999) e Biondi \& Baldoni (1995). Il bioclima dell'area in esame è di tipo Mediterraneo pluvistagionale oceanico con con termotipo termomediterraneo superiore ed ombrotipo subumido inferiore $\left(16^{\circ}\right.$ $\mathrm{C} \leq \mathrm{T} \leq 18^{\circ} \mathrm{C} ; 400<\mathrm{It} \leq 350 ; 600 \mathrm{~mm} \leq \mathrm{P} \leq 800 \mathrm{~mm}$ ). 


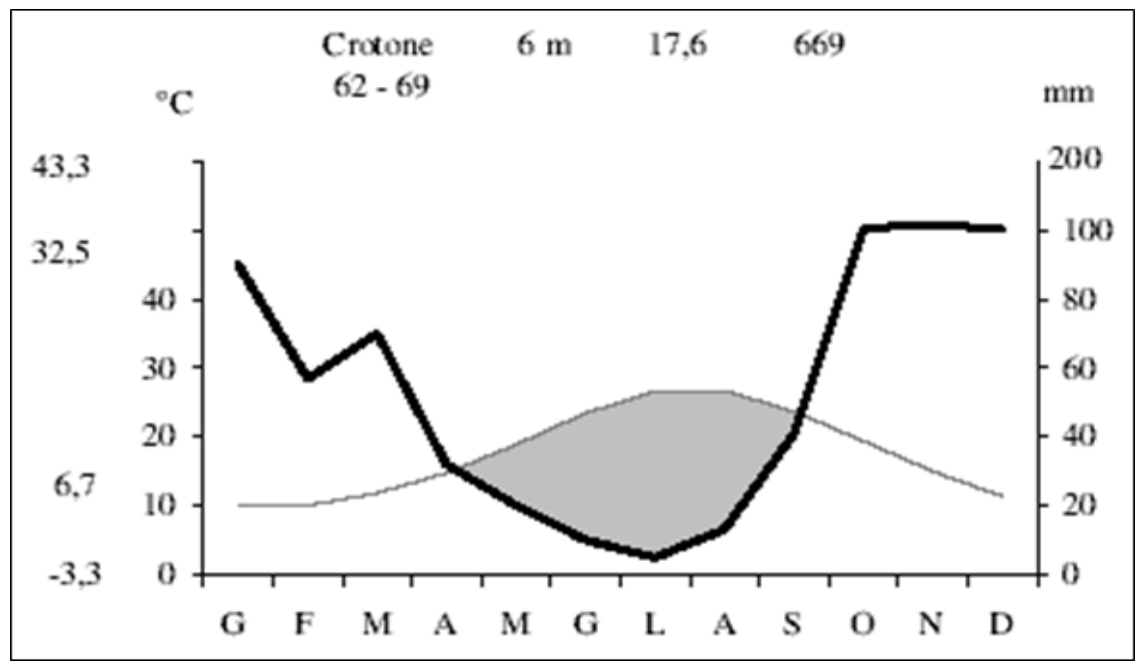

Fig. 10 Climogramma della stazione termopluviometrica di Crotone.

\subsection{Materiali e metodi}

Il rilevamento della vegetazione è stato effettuato seguendo il metodo fitosociologico sigmatista o di Zurigo-Montpellier (Braun-Blanquet, 1964; Westhoff \& Van Der Maarel 1978). Per la determinazione dei campioni è stato fatto riferimento a Pignatti (1982) e a Flora Europaea (Tutin et al., 1964-1980); la nomenclatura ha seguito Conti et al. (2005) e Celesti-Grapow et al. (2009a,b), ed è stata aggiornata in accordo con la Med-Checklist (Greuter et al.,1984-1989) e Euro+Med (2006-2018).

E' stato sviluppato un progetto GIS al fine di produrre una carta della vegetazione elaborata in scala 1:500, georiferita nel sistema di coordinate WGS 1984 UTM Zone 32N. Il software utilizzato per l'acquisizione e la gestione dell'informazione territoriale è ArcView (GIS) 10.2.

Le unità vegetazionali individuate sono state correlate con il sistema di classificazione degli habitat secondo la nomenclatura CORINE Biotopes (AA.VV., 1991) e secondo la Direttiva Habitat Natura 2000.

La qualità degli habitat è stata definita mediante due indicatori in grado di esprimere il valore naturale dell'habitat e il loro livello di conservazione. L'interpretazione del mosaico vegetazionale ha consentito di valutare per ogni unità di paesaggio individuata (biotopo) il grado di naturalità $(N)$, utilizzando l'Indice di naturalità degli habitat presentato nei materiali e metodi, e lo stato di conservazione ILC (Pizzolotto e Brandmayer, 1996), utilizzando protocolli consolidati in letteratura (Lausi et al., 1978; Poldini, Pertot, 1989; Villa. 1995; Ferrari et al., 2000; Ferrari et al., 2008; Cerabolini et al., 2007; Tomasella et al., 2007). Lo stato di conservazione dei biotopi, valutato applicando l'ILC ha tenuto conto delle classi di naturalità, individuate per gli habitat con l'indice precedente, e delle percentuali di copertura delle classi di utilizzo del suolo (Pizzolotto and Brandmayr, 1996).

\subsection{Risultati}

Tipologie vegetazionali, individuazione degli Habitat Natura 2000 e correlazione con CORINE Biotopes.

L'analisi geobotanica è stata condotta attraverso una campagna di sopralluoghi e rilevamenti vegetazionali e consultando la letteratura esistente per tipologie vegetazionali o ecologiche similari (Brullo et al., 1988; Brullo \& Spampinato, 1990; Maiorca \& Spampinato, 1999; Brullo et 
al., 2001; Brullo et al., 2002; Frondoni \& Iberite, 2002; Maiorca et al., 2002; Maiorca et al., 2005; Caridi et al., 2006; Maiorca et al., 2007; Spampinato et al., 2007), non essendo presenti studi specifici per l'area. e ha permesso di individuare 27 tipologie vegetazionali che sono state riportate nella Carta della Vegetazione elaborata per lo studio e restituita alla scala 1:10.000. Non è stato possibile cartografare sempre le singole fitocenosi o aspetti in mosaico che sono stati raggruppati nello stesso tipo fisionomico. Ogni tipologia è stata interpretata (Angelini et al., 2016) e, quindi, correlata con i sistemi di codifica a livello europeo come riportato nel testo dopo la descrizione della fitocenosi e sintetizzati in Tab 4. La distribuzione spaziale degli habitat di Direttiva e degli habitat CORINE è riportata nella Carta degli Habitat della Direttiva 92/43/CEE e nella Carta CORINE Biotopes in scala 1:10.000.

\section{SCHEMA SINTASSONOMICO}

PHRAGMITO-MAGNOCARICETEA Klika In Klika \& Novák 1941

PHRAGMITETALIA AUSTRALIS W. Koch 1926 em. Pignatti 1953

Phragmition australis W. Koch 1926 facies alofila

Polygono salicifolii-Phragmitetum communis Barbagallo, Brullo \& Furnari 1979

Phragmitetum communis (W. Koch 1926) Schmale 1939 (facies alofila)

Typhetum dominguensis Brullo, Minissale \& Spampinato 1994

Typho angustifoliae-Schoenoplectetum tabernaemontani Br.-Bl. \& Bolòs 1957

NASTURTIO-GLYCERIETALIA Pignatti 1953

Glycerio-Sparganion Br.-B1. \& Sissingh in Boer 1942

Sparganietum erecti (Roll. 1938) Philippi 1973

SCIRPETALIA COMPACTI Hejny in Holub, Hejny, Moravec \& Neuhäusl 1967 corr. Riv.Mart., Costa, Castroviejo \& Valdés 1980

1980

Scirpion compacti Dahl \& Hadac 1941 corr.Riv.-Mart., Costa, Castroviejo \& Valdés

Scirpetum compacti Van Langendonck 1931 corr. Bueno \& F. Prieto in Bueno 1997

JUNCETEA MARITIMI Br.-B1. In Br.-Bl., Roussine \& Nègre 1952

JUNCETALIA MARITIMI Br.-Bl. ex Horvatic 1934

Juncion maritimi Br.-Bl. ex Horvatic 1934

Juncetum acuti Molinier \& Tallon 1969

THERO-SUAEDETEA Rivas-Martínez 1972

THERO-SALICORNIETALIA EUROPAEAE Tx in Tx \& Oberd. ex Géhu \& Géhu-Franck 1984 Salicornion patulae Géhu \& Géhu-Franck 1984

Suaedo maritimae-Salicornietum patulae Brullo \& Furnari 1976 ex Géhu \& Géhu-Franck

1984

SAGINETEA MARITIMAE Westhoff, Van Leeuwen \& Adriani 1962

FRANKENIETALIA PULVERULENTAE Riv.-Mart. ex Castroviejo \& Porta 1976

Frankenion pulverulentae Riv.-Mart. ex Castroviejo \& Porta 1976

Spergularietum salinae Molinier \& Tallon 1969

STELLARIETEA MEDIAE Tüxen, Lohmeyer \& Preising ex von Rochov 1951

THERO-BROMETALIA (Riv.-God. et Riv.-Mart.ex Esteve 1973) O. Bolòs 1975

Echio plantaginei-Galactition tomentosae O. Bolòs \& Molinier 1969

Hordeion leporini Br.-Bl. in Br.-Bl., Gajewski, Wraber \& Walas 1936 corr. O. Bolòs 1962.

Centauretum napifoliae Brullo 1983 
CAKILETEA MARITIMAE Tüxen \& Preising Ex Br.-Bl \& Tüxen 1952

CAKILETALIA INTEGRIFOLIAE Tüxen ex Oberdorfer 1950 corr. Riv.-Mart., Costa \& Loidi 1992

Cakilion maritimae Pignatti 1953

Salsolo kali-Cakiletum maritimae Costa \& Mansanet 1981, corr. Rivas-Martínez et al. 1992

HELIANTHEMETEA GUTTATI (Br.-B1. In Br.-B1., Roussine \& Nègre 1952) Rivas Goday \& Rivas-Martinez 1963 Em. Rivas-Martinez 1978

MALCOLMIETALIA Riv.-God. 1958

Maresion nanae Géhu. Biondi, Géhu-Frank \& Arnold-Apostolides 1986

Sileno nicaeensis-Ononidetum variegatae Géhu et al. 1986

Laguro ovati-Vulpion membranaceae Géhu \& Biondi 1994

Sileno coloratae-Vulpietum membranaceae (Pign. 1953) Géhu \& Scoppola 1984

AMMOPHILETEA Br.-Bl. \& Tüxen Ex Westhoff, Dijk \& Passchier 1946

AMMOPHILETALIA Br.-Bl. (1931) 1933

Ammophilion australis Br.-B1. 1921 corr. Riv.-Mart., Costa \& Izco 1990

Echinophoro spinosae-Elytrigietum junceae Géhu 1988 corr. Géhu 1996

Echinophoro spinosae-Ammophiletum australis (Br.-Bl. 1933) Géhu, Rivas-Martinez \& R. Tx. 1972 in Géhu et al. 1984

CRUCIANELLETALIA MARITIMAE Sissingh 1974

Ononidion ramosissimae Pignatti 1952

Artemisio variabilis-Ephedretum distachyae Brullo, Giusso del Galdo, Siracusa \& Spampinato 2002

MOLINIO-ARRHENATHERETEA Tüxen 1937

PLANTAGINETALIA MAJORIS Tüxen \& Preising in Tüxen 1950

Trifolio fragiferi-Cynodontion Br.-Bl. \& O. Bolòs 1958

Trifolio fragiferi-Cynodontetum dactyli Br.-B1. \& O. Bolòs 1958

HOLOSCHOENETALIA VULGARIS Br.-B1. ex Tchou 1948

Paspalo distichi-Agrostion semiverticillatae Br.-B1. in Br.-B1., Roussine \& Nègre 1952

NERIO-TAMARICETEA BR.-BL. \& O. BÒLOS 1958

TAMARICETALIA AFRICANAE Br.-B1. \& O. Bòlos 1958 em. Izco, Fernández-Gonzáles \& Molina 1984

Tamaricion africanae Br.-B1. \& O. Bòlos 1958

Tamarici africanae-Viticetum agni-casti Brullo \& Spampinato 1997

Tamarici africanae-Viticetum agni-casti Brullo \& Spampinato 1997

ulmetosum minoris Brullo \& Spampinato 1997

POPULETEA ALBAE Br.-B1. 1962

POPULETALIA ALBAE Br.-B1. ex Tchou 1948

Populion albae Br.-Bl. 1931 ex Tchou 1948

Angelico sylvestris-Alnetum glutinosae Brullo et Spampinato 1997

Alno-Quercion roboris Horvat 1950

Clematido scandentis-Populetum albae (Brullo \& Spampinato 1997) Maiorca et al. nom. corr.

Fraxino-Quercetum roboris Gellini, Pedrotti \& Venanzoni 1986 
ALNETEA GLUTINOSAE Br.-B1. \& Tx. ex Westhoff et al. 1946

ALNETALIA GLUTINOSAE R.Tx. 1937

Salicion cinereae Müll. et Görs. 1958 p.p., Salicion auritae Doing 1962

Carici pendulae-Salicetum cinereae Brullo et Spampinato 1997

SALICI PURPUREAE-POPULETEA NIGRAE (Riv.-Mart. \& Cantó Ex Riv.-Mart., Báscones, T.E. Díaz, Fernández-Gonzáles \& Loidi) Riv.-Mart., Fernández-Gonzáles, Loidi, Lousa \& Penas 2001

SALICETALIA PURPUREAE Moor 1958

Salicion albae Soó 1930

Salicetum albo-brutiae Brullo \& Spampinato 1997

QUERCETEA ILICIS Br.-B1. in Br.-B1., Roussine \& Nègre 1952

PISTACIO LENTISCI-RHAMNETALIA ALATERNI Rivas-Martínez 1975

Oleo sylvestris-Ceratonion siliquae Br.-B1. ex Giunochet \& Drouineau 1944

Myrto-Pistacetum lentisci (Molinier 1954 em. O.Bolòs 1962) Rivas-Martinez 1975

Juniperion turbinatae Rivas-Martínez 1975 corr. 1987

Asparago acutifolii-Juniperetum macrocarpae (Molinier et R. Molinier 1955) O. Bolòs 1962

Entrando nel merito:

1. DUNE E RETRO-DUNE DEL LITORALE (CAKILETEA, AMMOPHILETEA, HELIANTHEMETEA)

Vegetazione pioniera alo-psammofila

- $\quad$ CAKILETEA MARITIMAE TÜXEN \& PREISING EX BR.-BL \& TÜXEN 1952

- $\quad$ Sintaxa fitosociologico: Salsolo kali-Cakiletum maritimae Costa \& Mansanet 1981, corr.

Rivas-Martínez et al. 1992

E' la vegetazione psammofila, pioniera, che forma la prima fascia di vegetazione discontinua dopo la zona afitoica prossima al mare. E' caratterizzata dalla presenza di varie terofite quali Cakile maritima, Salsola tragus ssp. pontica, Glaucium flavum, Echinophora spinosa, Eryngium maritimum, ecc. (Allegato IV Tab.1). Oltre alla subassociazione tipica (Allegato IV Tab. 1, ril. 1-3), in corrispondenza di accumulo di materiale organico si rinviene anche la subass. xanthietosum italici (Allegato IV Tab. 1, ril. 4-5).

Corrispondenza con $i$ sistemi di classificazione degli habitat a livello comunitario

Habitat Dir. 43/92 1210 - Vegetazione annua delle linee di deposito marine

Habitat CORINE 16.12 - Arenile con comunità vegetali annuali (CAKILETEA MARITIMAE)

Habitat EUNIS B1.131 - Comunità atlantico-mediterranee e del Mar Nero delle spiagge sabbiose

Land Cover Corine 3.3.1.2. - Vegetazione delle dune litoranee

Vegetazione delle dune embrionali

- $\quad$ AMMOPHILETEA BR.-BL. \& TÜXEN EX WESTHOFF, DIJK \& PASSCHIER 1946

- $\quad$ Sintaxa fitosociologico: Echinophoro spinosae-Elytrigietum junceae Géhu 1988 corr. Géhu 1996

E' una vegetazione erbacea a geofite ed emicriptofite, con bassi valori di copertura che colonizza le dune embrionali, mobili, modellate dal vento e dalle mareggiate. La cenosi è caratterizzata dalla presenza, e spesso dominanza, di Elymus farctus (= Elytrigia juncea), alla quale si associano 
Echinophora spinosa, Eryngium maritimum, Lotus creticus, Achillea maritima (= Otanthus maritimus) e Medicago marina. Nell'area indagata, un ruolo dominante è svolto da Otanthus maritimus, la cui diffusione è determinata dal disturbo antropico con passaggi di mezzi e fuoristrada (Allegato IV Tab. 2, ril. 6-14).

Corrispondenza con $i$ sistemi di classificazione degli habitat a livello comunitario

Habitat Dir. 43/92 2110 - Dune embrionali mobili

Habitat CORINE 16.2112 - Dune mobili embrionali del Mediterraneo

Habitat EUNIS B1.312 - Dune costiere mobili

Land Cover Corine 3.3.1.2 - Vegetazione delle dune litoranee

\section{Vegetazione delle dune mobili}

- $\quad$ AMMOPHILETEA BR.-BL. \& TÜXEN EX WeSthofF, DiJK \& PASSCHIER 1946

- $\quad$ Sintaxa fitosociologico: Echinophoro spinosae-Ammophiletum australis (Br.-Bl. 1933)

Géhu, Rivas-Martinez \& R. Tx. 1972 in Géhu et al. 1984

E' una cenosi che si rinviene in posizione più rialzata in mosaico o in contatto con la precedente cenosi. Si tratta di aspetti ascrivibili all'Echinophoro spinosae-Ammophiletum australis caratterizzati dalla dominanza di Ammophila littoralis (= A. australis), che riveste un ruolo importante nell'edificazione delle dune più elevate. A causa del disturbo antropico rilevato nell'area di studio, la formazione è estremamente localizzata e si rinviene nel tratto di spiaggia che si trova in sinistra idrografica rispetto alla foce del fiume Neto (Allegato IV Tab. 2, ril. 1516).

Corrispondenza con $i$ sistemi di classificazione degli habitat a livello comunitario

Habitat Dir. 43/92 2110 - Dune embrionali mobili

Habitat CORINE 16.2112 - Dune mobili embrionali del Mediterraneo

Habitat EUNIS B1.312 - Dune costiere mobili

Land Cover Corine 3.3.1.2 - Vegetazione delle dune litoranee

- HELIANTHEMETEA GUTTATI (BR.-BL. IN BR.-Bl., ROUSSINE \& NĖGRE 1952) RIVAS GODAY \& RIVAS-MARTINEZ 1963 EM. RIVAS-MARTINEZ 1978

- $\quad$ Sintaxa fitosociologico: Sileno nicaeensis-Ononidetum variegatae Géhu et al. 1986

Il Sileno nicaeensis-Ononidetum variegatae è una cenosi costituita da terofite scapose a fioritura tardo-primaverile. Si localizza sulle dune embrionali finché non viene sostituita dalle formazioni perenni, occupando i piccoli spazi aperti tra i cespi di Elymus farctus. Forma un mosaico con la vegetazione perenne dell'Echinophoro spinosae-Elytrigietum junceae (Allegato IV Tab. 3, ril. 17-18-19).

Corrispondenza con $i$ sistemi di classificazione degli habitat a livello comunitario

Habitat Dir. 43/92 2230 - Dune con prati dei Malcolmietalia

Habitat CORINE 16.228 - Comunità dunali mediterranee e sud-atlantiche di terofite su suolo sabbioso profondo

Habitat EUNIS B1.48 - Comunità dunali mediterranee e sud-atlantiche di terofite su suolo sabbioso profondo

Land Cover Corine 3.3.1.2 - Vegetazione delle dune litoranee

Vegetazione annuale psammofila delle dune

- HELIANTHEMETEA GUTTATI (BR.-BL. IN BR.-BL., ROUSSINE \& NÈGRE 1952)

RIVAS GODAY \& RIVAS-MARTINEZ 1963 EM. RIVAS-MARTINEZ 1978 
- $\quad$ Sintaxa fitosociologico: Sileno coloratae-Vulpietum membranaceae (Pign. 1953) Géhu \& Scoppola 1984

Negli spazi aperti dunali e nelle schiarite all'interno dei rimboschimenti a Pinus sp pl si rinviene una cenosi con carattere pionieristico e subnitrofilo, il Sileno coloratae-Vulpietum membranaceae. Si tratta di vegetazione erbacea annuale caratterizzata dalla dominanza di Vulpia fasciculata, Silene colorata, Silene nicaeensis e Medicago litoralis, completa il corteggio floristico un contingente di terofite sinantropiche (Allegato IV Tab. 3, ril. 20-21-22).

Corrispondenza con $i$ sistemi di classificazione degli habitat a livello comunitario

Habitat Dir. 43/92 2230 - Dune con prati dei Malcolmietalia

Habitat CORINE 16.228 - Comunità dunali mediterranee e sud-atlantiche di terofite su suolo sabbioso profondo

Habitat EUNIS B1.48 - Comunità dunali mediterranee e sud-atlantiche di terofite su suolo sabbioso profondo

Land Cover Corine 3.3.1.2 - Vegetazione delle dune litoranee

- $\quad$ AMMOPHILETEA BR.-BL. \& TÜXEN EX WeSTHOFF, DiJK \& PASSCHIER 1946

- Sintaxa fitosociologico: Artemisio variabilis-Ephedretum distachyae Brullo et al. 2002

$\mathrm{Su}$ limitate aree retrodunali, è possibile osservare in contatto con la vegetazione delle dune embrionali, aspetti relitti di vegetazione camefitica, riferibili all'Artemisio variabilis-Ephedretum distachyae, fitocenosi propria dei suoli sabbiosi compatti del retroduna. L'associazione è caratterizzata dalla dominanza di Ephedra distachya, rara nanofanerofita psammofila, presente in Italia solamente in Sardegna, Puglia, Basilicata e sulle coste ioniche calabresi. A Ephedra si associano Artemisia campestris subsp. variabilis, Cyperus capitatus e molte specie trasgressive dei MALCOLMIETALIA e CARTHAMETALIA (Allegato IV Tab. 4, ril. 23-24-25).

Corrispondenza con $i$ sistemi di classificazione degli habitat a livello comunitario

Habitat Dir. 43/92 2210 - Dune fisse del litorale (Crucianellion maritimae)

Habitat CORINE 16.223 - Dune grigie mediterraneo-Atlantiche

Habitat EUNIS B1.43 - Dune costiere stabili del Mediterraneo centrale e occidentale e delle coste termo-atlantiche del sud-Iberia e nord-Africa

Land Cover Corine 3.3.1.2 - Vegetazione delle dune litoranee

\section{Paludi subsalse del litorale a Scirpi e Giunchi (SCirpetalia CompaCti, JUNCETEA MARITIMI)}

Vegetazione palustre delle depressioni retrodunali

- $\quad$ SCIRPETALIA COMPACTI HEJNY 1967 EM. RH.-MART. 1980

- $\quad$ Sintaxa fitosociologico: Scirpetum compacti Van Langendonck 1931 corr. Bueno \& F. Prieto in Bueno 1997

Lo Scirpetum compacti s'insedia su substrati limoso-argillosi, localizzandosi nelle depressioni costiere inondate durante l'inverno. Durante la stagione estiva il prosciugamento determina un notevole aumento della concentrazione salina. Bolboschoenus maritimus (var. compactus) è la specie dominante cui si accompagnano altre specie trasgressive degli JUNCETEA MARITIMI (Allegato IV Tab. 5, ril. 26-27-28-29).

Corrispondenza con $i$ sistemi di classificazione degli habitat a livello comunitario

Habitat Dir. 43/92 1410 - Pascoli inondati mediterranei (JUNCETALIA MARITIMI)

Habitat CORINE 53.17 - Formazioni alofile a Bolboschoenus maritimus (= Scirpus maritimus)

Habitat EUNIS C3.27 - Comunità alofile di Scirpus, Bolboschoenus e Schoenoplectus 
Land Cover Corine 4.2.1.4 - Vegetazione a dominanza di canneti/giuncheti (zone umide costiere)

Vegetazione alo-igrofila ad emicriptofite

- JUNCETEA MARITIMI BR.-BL. IN BR.-BL., ROUSSINE \& NĖGRE 1952

- $\quad$ Sintaxa fitosociologico: Juncetum acuti Molinier \& Tallon 1970

E' una fitocenosi aloigrofila localizzata in posizione retrodunale ai bordi di depressioni con acque salmastre. Specie dominante è Juncus acutus seguito da altre specie degli JUNCETEA MARITIMI, come Tripolium pannonicum, Carex extensa, Juncus subulatus (Allegato IV Tab.6, ril. 30-31).

Corrispondenza con $i$ sistemi di classificazione degli habitat a livello comunitario

Habitat Dir. 43/92 1410 - Pascoli inondati mediterranei (JUNCETALIA MARITIMI)

Habitat CORINE 15.51 - Paludi salmastre mediterranee a Juncus maritimus

Habitat EUNIS A2.522 - Comunità mediterranee di Juncus maritimus e Juncus acutus di paludi salmastre

Land Cover Corine 4.2.1.4 - Vegetazione a dominanza di canneti/giuncheti (zone umide costiere)

\section{DEPRESSIONI CON SUOLI SALSI A DOMINANZA DI CHENOPODIACEAE ALO-NITROFILE} (THERO-SUAEDETEA, SAGINETEA MARITIMAE). Vegetazione alofila annuale estiva

- THERO-SUAEDETEA RIVAS-MARTÍNEZ 1972

- $\quad$ Sintaxa fitosociologico: Suaedo maritimae-Salicornietum patulae Brullo \& Furnari 1976 ex Géhu \& Géhu-Franck 1984

E' una cenosi erbacea a succulente annuali con carattere pioniero che colonizza le depressioni inondate da acque salse nei mesi invernali e secche precocemente durante l'estate. Predilige suoli sabbioso-limosi, ricchi di sostanze organiche. Salicornia patula è la specie dominante cui si associano Suaeda maritima e Salsola soda (Allegato IV Tab. 7, ril. 32-33).

Corrispondenza con $i$ sistemi di classificazione degli habitat a livello comunitario

Habitat Dir. 43/92 1310 Vegetazione annua pioniera a Salicornia e altre specie delle zone fangose e sabbiose

Habitat CORINE 15.113 - Comunità mediterranee a Salicornia

Habitat EUNIS A2.5513 - Comunità pioniere di Salicornia sp. delle paludi salse

Land Cover Corine 4.2.1. - Paludi salmastre

Vegetazione alo-nitrofila annuale primaverile

- SAGINETEA MARITIMAE WESTHOFF, VAN LEEUWEN \& ADRIANI 1962

- $\quad$ Sintaxa fitosociologico: Spergularietum salinae Molinier \& Tallon 1969

E' una vegetazione xerofila e alo-tollerante, che si insedia substrati compatti, tipica degli ambienti salmastri e spesso sottoposti a pascolamento o calpestati. La cenosi è caratterizzata da Spergularia salina (= Spergularia marina) alla quale si associano Hordeum maritimum, Frankenia pulverulenta, Plantago coronopus, Sphenopus divaricatus (Allegato IV Tab. 8, ril. 34-35).

Corrispondenza con $i$ sistemi di classificazione degli habitat a livello comunitario 
Habitat Dir. 43/92 1310 - Vegetazione annua pioniera a Salicornia e altre specie delle zone fangose e sabbiose

Habitat CORINE 15.12 - Comunità alonitrofile a Frankenia

Habitat EUNIS E6.13 - Comunità erbacee alofile pioniere dell'entroterra mediterraneo

Land Cover Corine 3.2.1.3 - Aree a pascolo naturale e praterie

\section{DEPRESSIONI PALUSTRI CON ACQUE SUBSALSE O DOLCI (PHRAGMITETALIA} AUSTRALIS, NASTURTIO-GLYCERETALIA)

Vegetazione palustre ad elofite

- PHRAGMITO-MAGNOCARICETEA KLIKA IN KLIKA \& NOVÁK 1941

- $\quad$ Sintaxa fitosociologico: Polygono salicifolii-Phragmitetum communis Barbagallo, Brullo \& Furnari 1979

Presso la sponda idrografica sinistra della foce del Fiume Neto e lungo alcuni i canali di drenaggio, caraterizati da acque eutrofiche stagnanti, sono diffusi canneti legati ad acque dolci a dominanza di Phragmites australis, cui si accompagnano poche altre specie igrofile quali Persicaria decipiens, Sparganium erectum subsp. neglectum, Iris pseudacorus. Questi canneti sono stati inquadrati nel Polygono salicifolii-Phragmitetum communis Barbagallo, Brullo \& Furnari 1979 (Allegato IV Tab. 9, ril. 36-37-38).

Corrispondenza con $i$ sistemi di classificazione degli habitat a livello comunitario

Habitat Dir. 43/92 non previsto

Habitat CORINE 53.11 - Canneti a Phragmites australis

Habitat EUNIS typicum: C3.2111 - Comunità dulciacquicole di Phragmites australis

Land Cover Corine 4.1.1.1. -Vegetazione a dominanza di canneti/giuncheti (zone umide fluviali)

- PHRAGMITO-MAGNOCARICETEA KLIKA IN KLIKA \& NOVÁK 1941

- $\quad$ Sintaxa fitosociologico: Phragmitetum communis (Koch 1926) Schmale 1939

Sono i canneti più diffusi, dominati da Phragmites australis e formanti cenosi quasi monospecifiche. La formazione rappresenta una facies più alofila della precedente ed è caratterizzata anche da Agrostis stolonifera e Cynanchum acutum (Allegato IV Tab. 10, ril. 3940-41).

Corrispondenza con $i$ sistemi di classificazione degli habitat a livello comunitario

Habitat Dir. 43/92 non previsto

Habitat CORINE 53.11 - Canneti a Phragmites australis

Habitat EUNIS typicum: C3.2111 - Comunità dulciacquicole di Phragmites australis

Land Cover Corine 4.1.1.1. -Vegetazione a dominanza di canneti/giuncheti (zone umide fluviali)

\section{- PHRAGMITO-MAGNOCARICETEA KLIKA IN KLIKA \& NOVÁK 1941}

- $\quad$ Sintaxa fitosociologico: Typhetum dominguensis Brullo, Minissale \& Spampinato 1994

La cenosi è formata da piccoli nuclei riscontrabili alla foce del Neto in destra idrografica e dominata da Typha dominguensis (= T. angustifolia subsp. australis). Si rinviene anche all'interno di fossati e canali, su suoli alluvionali argillosi, ricchi in sostanza organica, sommersi da acque poco profonde, stagnanti, da paludosi a subsalsi. A Typha si accompagnano poche altre specie come Persicaria decipiens e Lythrum salicaria (Allegato IV Tab. 11, ril. 42-43). 
Corrispondenza con i sistemi di classificazione degli habitat a livello comunitario

Habitat Dir. 43/92 non previsto

Habitat CORINE 53.13 - Formazioni a Typha sp.pl.

Habitat EUNIS C3.232 - Comunità di Typha angustifolia

Land Cover Corine 4.1.1.1. - Vegetazione a dominanza di canneti/giuncheti (zone umide fluviali)

- $\quad$ PHRAGMITO-MAGNOCARICETEA KLIKA IN KLIKA \& NOVÁK 1941

- $\quad$ Sintaxa fitosociologico: Typho angustifoliae-Schoenoplectetum tabernaemontani Br.-B1. \& Bolòs 1957

La cenosi s'insedia su substrati limoso-argillosi costantemente umidi. Si riscontra in destra idrografica alla foce del Fiume Neto. La specie dominante rimane Typha angustifolia cui si accompagna Schoenoplectus tabaernaemontani (Allegato IV Tab. 12, ril. 44-45-46).

\section{Corrispondenza con $i$ sistemi di classificazione degli habitat a livello comunitario}

Habitat Dir. 43/92 non previsto

Habitat CORINE 53.17 - Comunità alofile di Scirpus, Bolboschoenus e Schoenoplectus

Habitat EUNIS C3.232 - Comunità di elofite di grandi dimensioni e canneti marginali (Typha angustifolia)

Land Cover Corine 4.1.1.1. - Vegetazione a dominanza di canneti/giuncheti (zone umide fluviali)

- $\quad$ PHRAGMITO-MAGNOCARICETEA KLIKA IN KLIKA \& NOVÁK 1941

- $\quad$ Sintaxa fitosociologico: Sparganietum erecti (Roll. 1938) Philippi 1973

La cenosi più o meno paucispecifica colonizza ampie superfici tendenti all'impaludamento. Si ritrova in prossimità di stagni poco profondi o all'interno dei canali. La specie dominante è Sparganium erectum, a cui si accompagnano Berula erecta, Carex otrubae, Schoenoplectus tabernaemontani, Cyperus longus ssp. badius, Festuca arundinacea, ecc. (Allegato IV Tab. 13, ril. 47-48-49).

Corrispondenza con $i$ sistemi di classificazione degli habitat a livello comunitario

Habitat Dir. 43/92 non previsto

Habitat CORINE 53.14 - Comunità di medie dimensioni (Sparganium sp.pl.)

Habitat EUNIS typicum: C3.2 - Comunità di elofite di grandi dimensioni e canneti marginali Land Cover Corine 4.1.1.1. - Vegetazione a dominanza di canneti/giuncheti (zone umide fluviali)

\section{PraTi UMIDI (MOLINIO-ARRHENATHERETEA TÜXEN 1937) Praterie igrofile}

- $\quad$ PLANTAGINETALIA MAJORIS TÜXEN \& PREISING IN TÜXEN 1950

- $\quad$ Sintaxa fitosociologico: Trifolio fragiferi-Cynodontetum dactyli Br.-B1. \& O. Bolòs 1958

E' una fitocenosi che colonizza le aree poste in posizione più elevata e per questo sottoposte a coltura o a pascolo. Sono presenti numerose specie di trifogli (Trifolium fragiferum, Trifolium fragiferum, T. repens, T. tomentosum) insieme a specie trasgressive dei CARTHAMETALIA LANATI (Allegato IV Tab.14, ril. 50-51-52). In questi ambienti, è comune riscontrare aree costituite da popolamenti di Glycyrrhiza glabra.

Corrispondenza con $i$ sistemi di classificazione degli habitat a livello comunitario 
Habitat Dir. 43/92 6420 - Praterie umide mediterranee con piante erbacee alte del MolinioHoloschoenion

Habitat CORINE 37.4 - Prati umidi di erbe alte mediterranee

Habitat EUNIS E5.44 - Praterie mediterranee di aree fluviali alluvionali

Land Cover Corine 3.2.1.3 - Aree a pascolo naturale e praterie

6. BOSCHI IGROFILI E BOSCAGLIE RIPARIALI (POPULETALIA ALBAE, SALICETALIA PURPUREAE, PRUNETALIA SPINOSAE); CESPUGLIETI IMPALUdATI, SUBSAlSI, A DOMINANZA DI TAMERICI (NERIO-TAMARICETEA)

Cespuglieti edafo-igrofili eliofili

- $\quad$ NERIO-TAMARICETEA BR.-BL. \& O. BòlOS 1958

- Sintaxa fitosociologico: Tamarici africanae-Viticetum agni-casti Brullo \& Spampinato 1997

- Sintaxa fitosociologico: Tamarici africanae-Viticetum agni-casti Brullo \& Spampinato 1997 ulmetosum minoris Brullo \& Spampinato 1997

E' una fitocenosi arbustiva che raggiunge i $5 \mathrm{~m}$ di altezza e che si presenta frammentata all'interno di tutta l'area di studio. E'caratterizzata dalla dominanza di Vitex agnus-castus, cui si associano Tamarix africana, Prunus spinosa, Rubus ulmifolius, Rosa sempervirens, Vitis sylvestris. Localmente è stata osservata anche la sub associazione ulmetosum minoris, (Brullo \& Spampinato, 1997), che risulta meno igrofila e nelle quali assumono una certa rilevanza le specie provenienti dai boschi planiziali dei POPULETALIA ALBAE. Si tratta di una cenosi dinamicamente collegata con il bosco edafoigraofilo a Salix alba e Populus alba, un tempo esteso anche nelle aree oggi coltivate e che è possibile osservare lungo le sponde del fiume Neto. Nelle stazioni retrodunali periodicamente impaludate è possibile riscontrare la subassociazione ulmetosum minoris, nella quale è assente Tamarix africana mentre assume un ruolo fisionomicamente rilevante Ulmus minor, al quale si accompagnano specie trasgressive dei Populetalia (Allegato IV Tab. 15, ril. 53-54-55-56-57-58-59-60-61).

Corrispondenza con $i$ sistemi di classificazione degli habitat a livello comunitario

Habitat Dir. 43/92 92D0 - Gallerie e forteti ripari meridionali (NERIO-TAMARICETEA e Securinegion tinctoriae)

Habitat CORINE 44.8131 - Cespuglieti a Tamarice del Mediterraneo occidentale

Habitat EUNIS F9.312 - Bordure ripariali di Vitex agnus-castus

Land Cover Corine 3.2.2.2- Pseudomacchia

\section{Cespuglieti ripariali igrofili}

- $\quad$ Populion albae BR.-BL. EX TCHOU 1948

Sintaxa fitosociologico: Aggruppamento a Ulmus minor e Vitex agnus-castus

Sparse nel territorio di studio sono presenti situazioni cenologiche tali da non poter essere considerate idonee per il rilevamento fitosociologico. Si tratta di comunità formate da specie arboreo-arbustive ad attitudini igrofile che non formano un popolamento elementare ben identificabile. Queste comunità sono dominate da Ulmus minor e Vitex agnus-castus.

Corrispondenza con $i$ sistemi di classificazione degli habitat a livello comunitario

Habitat Dir. 43/92 92A0 - Foreste a galleria di Salix alba e Populus alba

Habitat CORINE 44.141 - Gallerie di salici bianchi mediterranei

Habitat EUNIS G1.1121 - Foreste a galleria mediterranee a Salice bianco

Land Cover Corine 3.1.1.6.- Saliceti e altre formazioni riparie 
- $\quad$ Sintaxa fitosociologico: Salicetum albo-brutiae Brullo \& Spampinato 1997

E' una fitocenosi a carattere pioniero localizzata lungo il tratto terminale del fiume Neto. Presenta una struttura alto-arbustiva molto densa, che vede la dominanza di Salix alba, a cui seguono, Salix purpurea subsp. lambertiana e Salix brutia, specie endemica della Calabria (Allegato IV Tab. 16, ril. 62-63-64).

Corrispondenza con $i$ sistemi di classificazione degli habitat a livello comunitario

Habitat Dir. 43/92 92A0 - Foreste a galleria di Salix alba e Populus alba

Habitat CORINE 44.141 - Gallerie di salici bianchi mediterranei

Habitat EUNIS G1.1121 - Foreste a galleria mediterranee a Salice bianco

Land Cover Corine 3.1.1.6. - Saliceti e altre formazioni riparie

\section{- SALICETALIA PURPUREAE MOOR 1958}

- $\quad$ Sintaxa fitosociologico: Carici pendulae-Salicetum cinereae Brullo \& Spampinato 1997

Si tratta di una cenosi a struttura alto-arbustiva con dominanza fisionomica di Salix cinerea, specie a portamento cespitoso, cui si accompagnano con ruolo subordinato Populus alba, Salix alba e Ulmus minor. Per la densa copertura di S. cinerea, e per le particolari condizioni edafiche, acque stagnanti e profonde, le specie erbacee e lianose sono scarsamente rappresentate. Fra queste sono presenti Carex pendula, Phragmithes australis, Iris pseudacorus, Tamus communis (Allegato IV Tab. 17, ril. 64-65-66-67-68).

Corrispondenza con $i$ sistemi di classificazione degli habitat a livello comunitario

Habitat Dir. 43/92 92A0 - Foreste a galleria di Salix alba e Populus alba

Habitat CORINE 44.141 - Gallerie di salici bianchi mediterranei

Habitat EUNIS G1.1121 - Foreste a galleria mediterranee a Salice bianco

Land Cover Corine 3.1.1.6.- Saliceti e altre formazioni riparie

\section{Boschi igrofili}

- $\quad$ Populion albae BR.-BL. EX TCHOU 1948

- $\quad$ Sintaxa fitosociologico: Angelico sylvestris-Alnetum glutinosae Brullo \& Spampinato 1997 iridetosum pseudacori Brullo \& Spampinato 1997

E' il bosco di Ontano con Alnus glutinosa come specie fisionomicamente dominante. La cenosi è legata a stazioni palustri e si insedia su aree pianeggianti costiere, aperte, su suoli ricchi in limo e argilla, in corrispondenza del tratto terminale del fiume Neto. Lo strato arboreo è costituito con ruolo subordinato da Salix alba, Populus alba, Populus nigra; nello strato arbustivo, sono presenti: Clematis vitalba, Vitis sylvestris, Rubus ulmifolius, Sambucus nigra, Cornus sanguineus, Euonimus europaeus, ecc. La presenza di Clematis viticella e Iris pseudacorus sono differenziali della sub associazione indicata come iridetosum pseudacori, riferita agli alneti planiziali della Calabria, attualmente rilevati solo presso la foce dei fiumi Neto e Crati (Brullo \& Spampinato, 1997). Quest'aspetto può rimanere tale se permangono le condizioni di impaludamento o evolvere verso boschi planiziari, rappresentati nell'area di studio dal Clematido viticellae-Populetum albae (Allegato IV Tab. 18, ril. 69-70-71).

Corrispondenza con $i$ sistemi di classificazione degli habitat a livello comunitario

Habitat Dir. 43/92 92A0 - Foreste a galleria di Salix alba e Populus alba

Habitat CORINE 44.51 - Gallerie meridionali di ontano nero

Habitat EUNIS G1.41 - Boschi palustri di Alnus sp. non su terreno acido torboso 
Land Cover Corine 3.1.1.6- Saliceti e altre formazioni riparie

- $\quad$ Alno-Quercion roboris HORVAT 1950

- Sintaxa fitosociologico: Clematido viticellae-Populetum albae Brullo \& Spampinato 1997

E' un bosco planiziare caratterizzato da Populus alba che occupa superfici palustri non direttamente interessati dalla dinamica fluviale. Si tratta di formazioni relitte, poste in posizione retrodunali e soggetti a periodi di sommersione durante i mesi invernali. Populus alba è specie dominante cui si associano Alnus glutinosa, Ulmus minor, Ficus carica. Nello strato arbustivo, rado, sono presenti Ligustrum vulgare, Rosa canina, Cornus sanguineus e specie lianose come Vitis vinifera ssp sylvestris, Clematis viticella, Solanum dulcamara, Hedera helix, ecc. (Allegato IV Tab. 19, ril. 72-73-74-75).

Corrispondenza con $i$ sistemi di classificazione degli habitat a livello comunitario

Habitat Dir. 43/92 92A0 - Foreste a galleria di Salix alba e Populus alba

Habitat CORINE 44.614 - Boscaglie a galleria di pioppo italico

Habitat EUNIS G1.314 - Boscaglie a galleria italiane a Populus sp.

Land Cover Corine 3.1.1.6 - Boschi a prevalenza di specie igrofile (boschi a prevalenza di salici e/o pioppi e/o ontani etc)

- $\quad$ Alno-Quercion roboris HORVAT 1950

- $\quad$ Sintaxa fitosociologico: Fraxino-Quercetum roboris Gellini, Pedrotti \& Venanzoni 1986

In sinistra idrografica rispetto la foce del Fiume Neto e in posizione retrodunale, sono presenti lembi di un bosco planiziario caratterizzato da Fraxinus oxycarpa, Quercus robur, Ulmus minor e da specie lianose tra cui Vitis vinifera ssp sylvestris, Tamus communis, Clematis vitalba, Smilax aspera. Fra le specie arbustive si rinvengono Rosa canina, Ligustrum vulgare, Rhamnus alaternus, Pistacia lentiscus. Lo strato erbaceo presenta Brachypodium sylvaticum, Iris fetidissima, Arum italicum, Carex divulsa, Rumex sanguineus, ecc. (Allegato IV Tab. 20, ril. 7677-78-79-80-81-82-83).

Corrispondenza con $i$ sistemi di classificazione degli habitat a livello comunitario

Habitat Dir. 43/92 91F0 - Foreste miste riparie di grandi fiumi a Quercus robur, Ulmus laevis e Ulmus minor, Fraxinus excelsior o Fraxinus angustifolia (Ulmenion minoris)

Habitat CORINE 44.44 - Foreste miste a querce olmi e frassini dei grandi fiumi

Habitat EUNIS G1.2 - Boschi e foreste ripariali di Fraxinus - Alnus sp. o Quercus - Ulmus Fraxinus sp.

Land Cover Corine 3.1.1.6 - Bosco costiero dei suoli idrici

\section{MACCHIE E GARIGHE (QUERCETEA ILICIS, CISTO-MICROMERIETEA)}

- Juniperion turbinatae RIVAS-MARTÍNEZ 1975 CORR. 1987

- $\quad$ Sintaxa fitosociologico: Asparago acutifolii-Juniperetum macrocarpae

E' una macchia psammofila tipica delle dune stabilizzate, dominata da Juniperus macrocarpa (= Juniperus oxycedrus subsp. macrocarpa) e Juniperus phoenicea subsp. turbinata, che si rinviene in sinistra idrografica rispetto il Fiume Neto e collocata nelle porzioni maggiormente elevate della duna. Costituisce l'aspetto più evoluto di una serie edafo-climacica che svolge un importante ruolo nei processi di stabilizzazione delle dune costiere interessate da comunità degli AMMOPHILETEA. Si tratta di una macchia attualmente molto frammentata e ridotta a lembi 
relitti, in cui a Juniperus sp pl si accompagnano Pistacia lentiscus, Myrtus communis, Tamus communis (Allegato IV Tab. 21, ril. 84-85-86-87-88-89-90-91).

Corrispondenza con $i$ sistemi di classificazione degli habitat a livello comunitario

Habitat Dir. 43/92 2250* - Dune costiere con Juniperus spp.

Habitat CORINE 16.27 - Ginepreti e cespuglieti delle dune

Habitat EUNIS B1.63 - Comunità arbustive di Juniperus sp. su dune costiere

Land Cover Corine 3.2.3.1 - Macchia alta

- PISTACIO LENTISCI-RHAMNETALIA ALATERNI RIVAS-MARTÍNEZ 1975

- $\quad$ Sintaxa fitosociologico: Myrto-Pistacietum lentisci (Molinier 1954 em. O. Bolos 1962) Rivas-Martinez 1975

E' una macchia sclerofilla caratterizzata da Pistacia lentiscus che occupa i cordoni dunali più interni, in seguito ad una consistente stabilizzazione del substrato. La formazione si rinviene in maniera frammentaria anche nelle aree più interne lasciate libere dai coltivi, dove sono presenti elementi del Tamarici africanae-Viticetum agni-casti. Dal punto di vista fisionomico, la cenosi è caratterizzata da uno strato arbustivo alto 1.5-3.0 m, impenetrabile, dominato da Pistacia lentiscus cui si associano Phillyrea latififolia, Myrtus communis e Olea europaea var. sylvestris, Smilax aspera, Tamus communis (Allegato IV Tab. 22, ril. 92-93-94-95-96).

Corrispondenza con $i$ sistemi di classificazione degli habitat a livello comunitario

Habitat Dir. 43/92 2260 - Dune con vegetazione di sclerofille dei CISTO-LAVANDULETALIA

Habitat CORINE 16.28 - Cespuglieti a sclerofille delle dune

Habitat EUNIS B1.64 - Comunità arbustive di sclerofille e laurifille su dune costiere

Land Cover Corine 3.2.3.1 - Macchia alta

8. RIMBOSCHIMENTI, INCOLTI, COLTIVI, AMBIENTI FORTEMENTE ANTROPIZZATI (STELLARIETEA MEDIAE, PAPAVERETEA RHOEADIS, ONOPORDETEA ACANTHI, POLYGONO-POETEA ANNUAE)

Rimboschimenti

Nell'area di studio sono presenti due importanti rimboschimenti effettuati intorno agli anni ' 50 e poi '90, costituiti da Pinus sp pl, Eucaliptus sp pl ed altre specie esotiche. In questi contesti, la vegetazione non si esprime secondo le potenzialità del sito. Quello che è visibile è una fascia arborata con evidente sesto d'impianto, parallela alla linea di costa, cui si accompagnano aspetti di vegetazione erbacea annuale di tipo ruderale ricchi di specie prive di significato fitogeografico dei MALCOLMIETALIA e dell'Alkanno-Malcolmion ramosissimae, quali Bromus rigidus, Cutandia maritima, Lagurus ovatus, pseudorlaya pumila, Ononis variegata, Silene nicaeensis (Allegato IV Tab. 25, ril. 102-103).

Corrispondenza con $i$ sistemi di classificazione degli habitat a livello comunitario Habitat Dir. 43/92 -

Habitat CORINE 83.31/83.322 - Piantagioni di conifere/Piantagione di eucalipti Habitat EUNIS G3.F1 - Rimboschimenti e piantagioni altamente artificiali di conifere Land Cover Corine 3.1.2.1- Boschi a prevalenza di pini meditterranei (pino domestico, pino marittimo) e cipressete

Vegetazione sinantropica

- $\quad$ THERO-BROMETALIA (RIV.-GOD. ET RIV.-MART.EX ESTEVE 1973) O. BOlÒs 1975 
Si tratta di una vegetazione molto diffusa nel territorio in esame. Le cenosi presenti possono essere inquadrate in due alleanze: Echio plantaginei-Galactition tomentosae e Hordeion leporini. La prima alleanza riunisce la vegetazione a ciclo primaverile rilevabile negli incolti ed ex coltivi, con un ricco contingente di piante annuali di tipo subnitrofilo, fra cui spiccano Bromus madritensis, Galactites tomentosa, Echium italicum, ecc. Particolarmente diffusa è la vegetazione commensale delle colture a grano con Ridolfia segetum, Daucus muricatus, Phalaris paradoxa e Ammi majus, specie fisionomicamente dominanti che si uniscono a un discreto contingente di specie dell'Hordeion leporini. Le cenosi tipiche dei margini stradali, anche molto antropizzati, rientrano nell'Hordeion leporini, e si localizzano su suoli aridi, ben drenati e compatti, spesso adiacenti le carraie. Le specie dominanti osservate sono Avena barbata e Bromus diandrus subsp. maximus.

- $\quad$ Hordeion leporini BR.-BL. IN BR.-BL., GAJEWSKI, WRABer \& WALAS 1936 CORR. O. BoLÒs 1962.

- $\quad$ Sintaxa fitosociologico: Centauretum napifoliae Brullo 1983

E' una formazione subnitrofila emicripto-terofitica, che si rinviene comunemente sui suoli argillosi di natura alluvionale delle pianure costiere e che è frequente riscontrare lungo strade, sentieri ed ambienti ruderali in genere. E'caratterizzata floristicamente e fisionomicamente da Centaurea napifolia, Glycyrrhiza glabra e da specie dell'Hordeion leporini, Sisymbrietalia e Stellarietea mediae (Allegato IV Tab. 23, ril. 97-98-99).

- $\quad$ Hordeion leporini BR.-BL. IN BR.-BL., GAJEWSKI, WraBER \& WALAS 1936 CORR. O. BoLÒs 1962.

- $\quad$ Sintaxa fitosociologico: Aggr. a Dasypyrum villosum e Chondrilla juncea

In corrispondenza di bordi di strade sterrate e ambienti sinantropici con leggera nitrificazione del suolo, dovuta allo spargimento di concimi nei campi limitrofi, si osserva una vegetazione monostratificata a struttura chiusa, dominata da Chondrilla juncea, Critesion murinum ssp. leporinum, Dasypyrum villosum, Avena sterilis, Lagurus ovatus, Lophochloa cristata, Plantago lagopus (Allegato IV Tab. 24, ril. 100-101).

\section{Corrispondenza con $i$ sistemi di classificazione degli habitat a livello comunitario}

Habitat Dir. 43/92 -

Habitat CORINE 83.1 - Prati concimati e pascolati anche abbandonati e vegetazione post colturale

Habitat EUNIS I - Habitat rurali e domestici, con coltivazioni agricole ed orto-frutticole, attive o recenti

Land Cover Corine 2.1.1.1 - Colture Intensive

\subsection{Analisi dei Biotopi}

Ogni tipologia vegetazionale è stata associata ad una unità omogenea definita biotopo. I biotopi costituiscono porzioni del territorio percepibili come delle unità anche di tipo paesistico in quanto i loro confini fisici e geografici sono facilmente rilevabili e definiti dal resto del paesaggio. Essi contengono uno o più ambiti, caratterizzati o meno da habitat di valore naturalistico per la presenza di specie e comunità animali o vegetali di interesse in quanto rappresentative dell'ambiente naturale. Su base fisionomia-vegetazionale sono state rilevate le seguenti unità: 1 . Ambienti costieri (Biotopo delle coste sabbiose), 2. Pascoli (Biotopo dei pascoli alofili e 
subalofili), 3. Ambienti fluviali (Biotopo degli ambienti palustri, Biotopo dei boschi planiziali, Biotopo delle boscaglie e cespuglieti edafo-igrofili), 4. Macchia mediterranea (Biotopo della macchia), 5. Ambienti a determinismo antropico (Biotopo dei coltivi, Biotopo dei rimboschimenti) (Tab 4).

E' stata analizzata la corrispondenza dei biotopi individuati nell'area di studio con i sistemi di classificazione degli habitat a livello comunitario (Habitat Dir. 43/92, Corine biotopes e Habitat EUNIS).

Dall'analisi è emerso che il Biotopo delle coste sabbiose, presente con le fitocenosi Salsolo kaliCakiletum maritimae, Echinophoro spinosae-Elytrigietum junceae, Echinophoro spinosaeAmmophiletum australis, Artemisio variabilis-Ephedretum distachyae, Sileno colorataeVulpietum membranaceae, Sileno nicaeensis-Ononidetum variegatae, e da aspetti molto disturbati dei MALCOLMIETALIA/SYSIMBRIETALIA/STELLARIETEA, è rappresentato dagli habitat di Direttiva 1210, 2110, 2110, 2210, 2230, 2230, 2230.

Il Biotopo degli ambienti alofili, subalofili e dei prati umidi, presenti nelle depressioni salmastre è presente con lo Scirpetum compacti, Juncetum acuti, Suaedo maritimae-Salicornietum patulae, Spergularietum salinae, Trifolio fragiferi-Cynodontetum dactyli, aspetti inquadrati dalla Direttiva nel 1410, 1310 e 6420.

Il Biotopo degli ambienti palustri è presente con Polygono salicifolii-Phragmitetum communis, Phragmitetum communis, Typhetum dominguensis, Typho angustifoliae-Schoenoplectetum tabernaemontani, Sparganietum erecti. Questi aspetti non trovano corrispondenze con la Direttiva, ma sono riconducibili ai biotopi CORINE 53.11, 53.11, 53.13, 53.17, 53.14.

Il Biotopo dei boschi planiziali, boscaglie ripariali e dei cespuglieti edafo-igrofili è rappresentato dal Tamarici africanae-Viticetum agni-casti, Paspalo distichi-Agrostion semiverticillatae, Aggr. a Ulmus minor e Vitex agnus castus, Salicetum albo-brutiae, Carici pendulae-Salicetum cinereae, Angelico sylvestris-Alnetum glutinosae subass. iridetosum pseudacori, Clematido viticellaePopuletum albae e dal Fraxino-Quercetum roboris; questi aspetti sono riconducibili agli habitat 92D0, 3280, 91F0, 92A0, 91F0.

Il Biotopo della macchia è rappresentato da formazioni arbustive sparse nel territorio e da una macchia costiera a ginepro. Gli aspetti sono inquadrabili nell'Asparago acutifolii-Juniperetum macrocarpae e nel Myrto-Pistacietum lentisci; gli habitat di Direttiva corrispondenti sono il $2250 *$ e il 2260 (Tab. 4). 


\begin{tabular}{|c|c|c|c|c|c|c|}
\hline Biotopi & Fitocenosi & $\begin{array}{l}\text { Naturalit } \\
\text { à }\end{array}$ & $\begin{array}{c}\text { Habi } \\
\text { tat } \\
\text { Dir. } \\
\text { 43/92 }\end{array}$ & $\begin{array}{c}\text { Corine } \\
\text { biotopes } \\
(2003)\end{array}$ & $\begin{array}{l}\text { Habita } \\
\text { t } \\
\text { EUNIS } \\
(2017)\end{array}$ & $\begin{array}{c}\text { Corine } \\
\text { Land } \\
\text { Cover } \\
2012 \text { IV } \\
\text { Livello }\end{array}$ \\
\hline \multirow{7}{*}{$\begin{array}{l}\text { 1. Ambienti costieri } \\
\text { - Biotopo delle coste } \\
\text { sabbiose }\end{array}$} & $\begin{array}{l}\text { Salsolo kali-Cakiletum } \\
\text { maritimae }\end{array}$ & 5 & 1210 & 16.12 & B1.131 & 3.3 .1 .2 \\
\hline & $\begin{array}{l}\text { Echinophoro spinosae- } \\
\text { Elytrigietum junceae }\end{array}$ & 5 & 2110 & 16.2112 & B1.312 & 3.3 .1 .2 \\
\hline & $\begin{array}{l}\text { Echinophoro spinosae- } \\
\text { Ammophiletum australis }\end{array}$ & 5 & 2110 & 16.2112 & B1.312 & 3.3.1.2. \\
\hline & $\begin{array}{l}\text { Artemisio variabilis- } \\
\text { Ephedretum distachyae }\end{array}$ & 5 & 2210 & 16.223 & B1.43 & 3.3.1.2 \\
\hline & $\begin{array}{l}\text { Sileno coloratae- } \\
\text { Vulpietum membranaceae }\end{array}$ & 4 & 2230 & 16.228 & B1.48 & 3.3 .1 .2 \\
\hline & $\begin{array}{l}\text { Sileno nicaeensis- } \\
\text { Ononidetum variegatae }\end{array}$ & 4 & 2230 & 16.228 & B1.48 & 3.3 .1 .2 \\
\hline & $\begin{array}{l}\text { Malcolmietalia/Sysimbrie } \\
\text { talia/Stellarietea }\end{array}$ & 2 & 2230 & 16.1231 & B1.131 & 3.3 .1 .2 \\
\hline \multirow{5}{*}{$\begin{array}{l}\text { 2. Pascoli } \\
\text { - Biotopo dei pascoli } \\
\text { alofili e subalofili }\end{array}$} & Scirpetum compacti & 4 & 1410 & 53.17 & C3.27 & 4.2 .1 .4 \\
\hline & Juncetum acuti & 4 & 1410 & 15.51 & A2.522 & 4.2 .1 .4 \\
\hline & $\begin{array}{l}\text { Suaedo maritimae- } \\
\text { Salicornietum patulae }\end{array}$ & 4 & 1310 & 15.113 & $\begin{array}{c}\mathrm{A} 2.551 \\
3\end{array}$ & 4.2 .1 .4 \\
\hline & Spergularietum salinae & 2 & 1310 & 15.12 & E6.13 & 4.2 .1 .4 \\
\hline & $\begin{array}{l}\text { Trifolio } \quad \text { fragiferi- } \\
\text { Cynodontetum dactyli }\end{array}$ & 3 & 6420 & 37.4 & E5.44 & 3.2 .1 .3 \\
\hline \multirow{13}{*}{$\begin{array}{l}\text { 3. Ambienti fluviali } \\
\text { - Biotopo degli ambienti } \\
\text { palustri } \\
\text { - Biotopo dei boschi } \\
\text { planiziali, } \\
\text { - Biotopo delle boscaglie } \\
\text { e cespuglieti edafo- } \\
\text { igrofili }\end{array}$} & $\begin{array}{l}\text { Polygono } \quad \text { salicifolii- } \\
\text { Phragmitetum communis }\end{array}$ & 4 & - & 53.11 & $\begin{array}{c}\text { C3.211 } \\
1\end{array}$ & 4.1.1.1 \\
\hline & Phragmitetum communis & 4 & - & 53.11 & $\begin{array}{c}\mathrm{C} 3.211 \\
1 \\
\end{array}$ & 4.1.1.1 \\
\hline & Typhetum dominguensis & 4 & - & 53.13 & C3.232 & 4.1 .1 .1 \\
\hline & $\begin{array}{l}\text { Typho angustifoliae- } \\
\text { Schoenoplectetum } \\
\text { tabernaemontani }\end{array}$ & 4 & - & 53.17 & C 3.232 & 4.1.1.1 \\
\hline & Sparganietum erecti & 4 & - & 53.14 & $\mathrm{C} 3.2$ & 4.1 .1 .1 \\
\hline & $\begin{array}{l}\text { Tamarici africanae- } \\
\text { Viticetum agni-casti }\end{array}$ & 5 & 92D0 & 44.8131 & F9.312 & 3.2 .2 .2 \\
\hline & $\begin{array}{ll}\text { Paspalo distichi- } \\
\text { Agrostion } \\
\text { semiverticillatae }\end{array}$ & 3 & 3280 & 24.53 & E.544 & 3.1.1.6 \\
\hline & $\begin{array}{l}\text { Aggr. a Ulmus minor e } \\
\text { Vitex agnus castus }\end{array}$ & 3 & $91 \mathrm{~F} 0$ & 44.62 & G1.3 & 3.1.1.6 \\
\hline & Salicetum albo-brutiae & 5 & $92 \mathrm{~A} 0$ & 44.141 & $\begin{array}{c}\text { G1.112 } \\
1 \\
\end{array}$ & 3.1.1.6 \\
\hline & $\begin{array}{l}\text { Carici pendulae- } \\
\text { Salicetum cinereae }\end{array}$ & 5 & $92 \mathrm{~A} 0$ & 44.141 & $\begin{array}{c}\text { G1.112 } \\
1\end{array}$ & 3.1.1.6 \\
\hline & $\begin{array}{ll}\text { Angelico } & \text { sylvestris- } \\
\text { Alnetum } & \text { glutinosae } \\
\text { subass. } & \text { iridetosum } \\
\text { pseudacori } & \end{array}$ & 5 & $92 \mathrm{~A} 0$ & 44.51 & G1.41 & 3.1.1.6 \\
\hline & $\begin{array}{l}\text { Clematido viticellae- } \\
\text { Populetum albae }\end{array}$ & 5 & $92 \mathrm{~A} 0$ & 44.614 & G1.314 & 3.1.1.6 \\
\hline & $\begin{array}{l}\text { Fraxino-Quercetum } \\
\text { roboris }\end{array}$ & 5 & $91 \mathrm{~F} 0$ & 44.44 & G1.2 & 3.1.1.6 \\
\hline \multirow{2}{*}{ 4. Macchia mediterranea } & $\begin{array}{l}\text { Asparago acutifolii- } \\
\text { Juniperetum macrocarpae }\end{array}$ & 5 & $\begin{array}{c}2250 \\
*\end{array}$ & 16.27 & B1.63 & 3.2 .3 .1 \\
\hline & $\begin{array}{l}\text { Myrto-Pistacietum } \\
\text { lentisci }\end{array}$ & 5 & 2260 & 16.28 & B1.64 & 3.2 .3 .1 \\
\hline \multirow{2}{*}{$\begin{array}{l}\text { 5. Ambienti a } \\
\text { determinismo antropico } \\
\text { - Biotopo dei coltivi } \\
\text { - Biotopo dei } \\
\text { rimboschimenti }\end{array}$} & $\begin{array}{l}\text { Rimboschimenti a Pinus } \\
\text { sp pl e Eucaliptus sp pl }\end{array}$ & 2 & - & $\begin{array}{l}83.31 / \\
83.322 \\
\end{array}$ & B1.7 & 3.1 .2 .1 \\
\hline & $\begin{array}{l}\text { Vegetazione sinantropica } \\
\text { Thero-Brometalia }\end{array}$ & 1 & - & 83.1 & I & 2.1.1.1 \\
\hline
\end{tabular}

Tab. 4 Biotopi individuati nell'area di studio e corrispondenza con i sistemi di classificazione degli habitat a livello Comunitario 


\subsection{Qualità del Paesaggio}

Dall'analisi statistica risulta evidente come gli ambienti legati al determinismo antropico occupino la maggior parte dell'area, ricoprendo complessivamente il 50.46\%. I biotopi di maggior valore naturalistico sono quelli della macchia mediterranea con quasi il $4 \%$, dell'ambiente fluviale con $7.53 \%$ e delle coste sabbiose con oltre il $10 \%$.

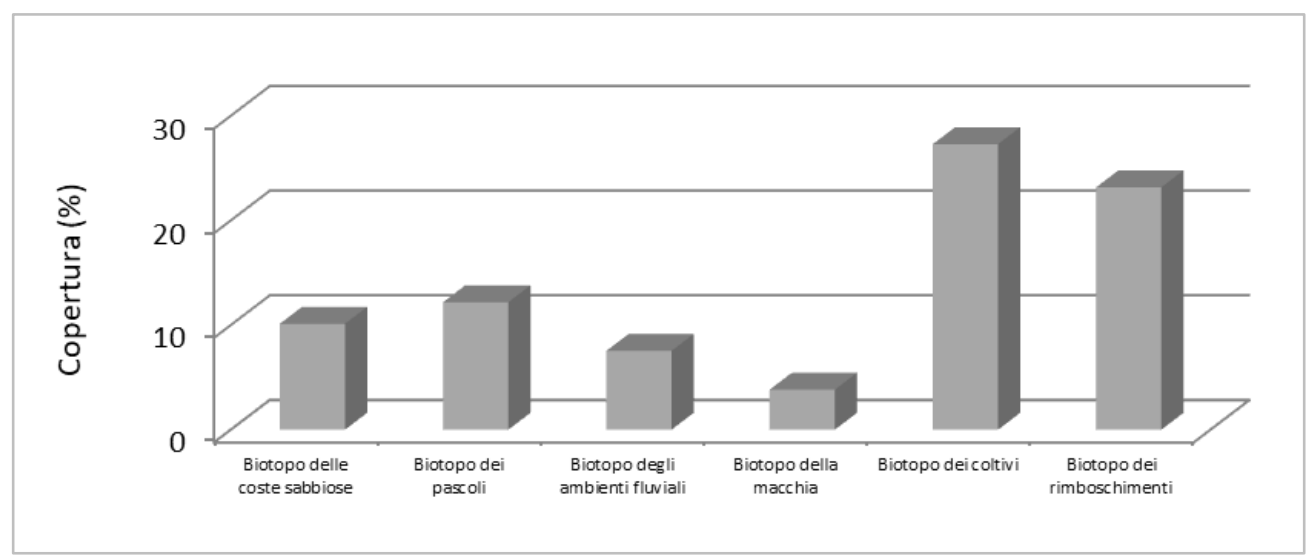

Naturalità - Attribuendo a ciascuna fitocenosi cartografata un valore di naturalità sulla base della posizione nella serie di vegetazione di pertinenza è stata realizzata la Carta della Naturalità del territorio in scala 1:10.000. Come risulta dalla distribuzione percentuale delle classi di naturalità, in oltre il 55\% dell'area sono presenti superfici destinate a colture (classe di naturalità 1) e impianti artificiali di pini ed eucalipti (classe di naturalità 2), che occupano gran parte della superficie potenzialmente interessata dalle formazioni climaciche. L'altra metà dell'area presenta aspetti di vegetazione naturale e seminaturale e, soprattutto, aspetti di degradazione di boschi planiziali, come testimoniato dalle rade chiazze di vegetazione arbustiva edafo-igrofila presenti. Le classi di maggior valenza naturalistica, classe 4 con $11.8 \%$ e classe 5 con $19.0 \%$, si riferiscono alle formazioni della macchia a ginepro e di quella a mirto e lentisco e al boschetto di Farnia e Frassino. Queste formazioni rappresentano, insieme alle boscaglie di salici e pioppi, che si insediano lungo il fiume, gli aspetti naturalistici più rilevanti dell'area.

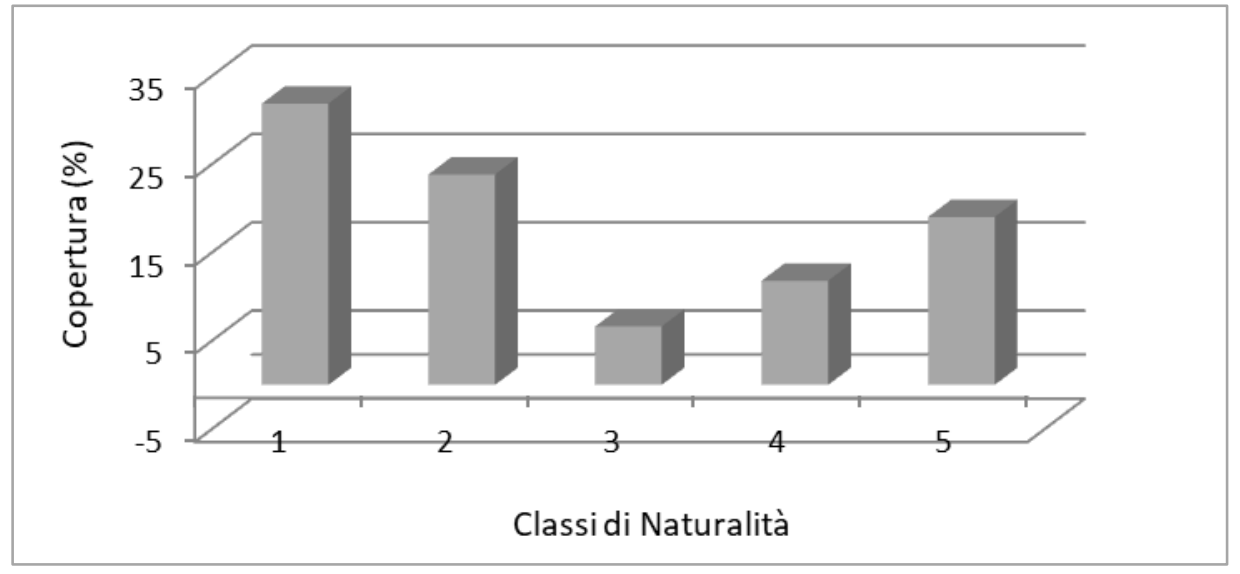

Conservazione - Per quantificare la qualità ambientale e lo stato di conservazione del paesaggio, è stato applicato l'indice di conservazione del paesaggio, ILC (Pizzolotto \& Brandmayer, 1996 ). L'indce può essere applicato a differenti scale territoriali, permettendo di definire lo stato di 
conservazione di una determinata unità ambientale individuata. Il calcolo dell' $I L C$ per l'intero SIC ha restituito il valore di 0.69 che, in una scala con range $0-1$, indica un medio stato di conservazione del SIC, indicando comunque che il sito necessita di strategie per superare il conflitto tra un utilizzo del suolo per fini agricoli ed economici da una parte e la conservazione delle risorse e dei servizi biologici dall'altra.

Il dato comunuque, seppur indicativo, non è sufficiente a specificare quale sia il punto di vulnerabilità dell'intero sistema.

Ci si chiede, cioè, quale sia nell'ambito del SIC Foce Neto l'unità paesistica più vulnerabile, dal punto di vista ecologico. A questo proposito è stata condotto un approfondimento per individuare lo stato di conservazione di aree omogenee, facilmente riconoscibili, la cui identità è svincolata sia dai criteri estetico-percettivi, sia dall'approccio storico-culturale. Si tratta di un approccio ecologico che assume il paesaggio inteso come "un mosaico di ecosistemi e di usi del suolo che si ripete secondo una configurazione spaziale riconoscibile su un'area più o meno estesa» (Forman e Godron, 1986).

Per mezzo della fitosociologia e della sinfitosociologia (fitosociologia seriale o dinamica) è possibile identificare ambiti territoriali potenzialmente atti a ospitare un solo tipo di vegetazione seriale (serie di vegetazione). Per effetto dell'antropizzazione nel mosaico paesistico reale questi elementi di vegetazione possono non essere presenti o presentarsi in forma frammentata, all'interno di una matrice paesistica costituita da differenti tipi di coltivazione e di pattern insediativo (Barbati \& Chirici, 2009). Gli strumenti d'analisi dell'ecologia del paesaggio su base fitosociologica hanno consentito di identificare tre ambiti territoriali omogenei (Unità ambientali): Unità paesaggistica degli ambienti fluviali, Unità paesaggistica degli ambienti planiziali, Unità paesaggistica degli ambienti dunali costieri. Queste Unità ambientali rappresentano le unità paesaggistico-ecologiche del mosaico territoriale all'interno del SIC, rispetto le quali articolare indicazioni e indirizzi di tutela, recupero e valorizzazione del paesaggio. La qualità ambientale e lo stato di conservazione delle Unità ambientali è stata quantificata in modo speditivo applicando l'ILC.

I valori ottenuti evidenziano che l'Unità paesaggistica degli ambienti fluviali versa in uno stato di conservazione migliore con un $I L C=0.76$, rispetto le altre due unità. Sebbene le coltivazioni si estendano fino agli argini del fiume, è possibile individuare la geoserie della vegetazione dulciacquicola planiziaria. L'Unità paesaggistica degli ambienti dunali costieri presenta un ILC= 0.68. E' possibile identificare gli stadi della geoserie della vegetazione psammofila, sebbene le cenosi siano fortemente disturbate dalla presenza dei rimboschimenti; il punto di fragilità del sistema è rappresentato dall'Unità paesaggistica degli ambienti planiziali con $I L C=0.20$, in cui insistono le attività agricole.

Sulla base dei dati derivati dalla Carta della Vegetazione, della Carta della Naturalità e dei valori dell'ILC è stata proposta una zonizzazione del SIC. La zonizzazione è opportuna come strumento tutorio e gestionale per individuare le priorità degli interventi proposti nella gestione del SIC.

Il sito è stato distinto in tre zone $a, b, c$ : la zona $a$ include il fiume e le aree dunali costiere, rappresenta l'area dalla valenza naturalistica più elevata; la zona $b$ comprende la superficie occupata dai rimboschimenti, le isole di vegetazione edafoigroila e costituisce un'area buffer rispetto la zona a; ha valenza naturalistica intermedia. La zona $c$ include le superfici coltivate $\mathrm{e}$ rappresenta le aree a valenza naturalistica più bassa. 


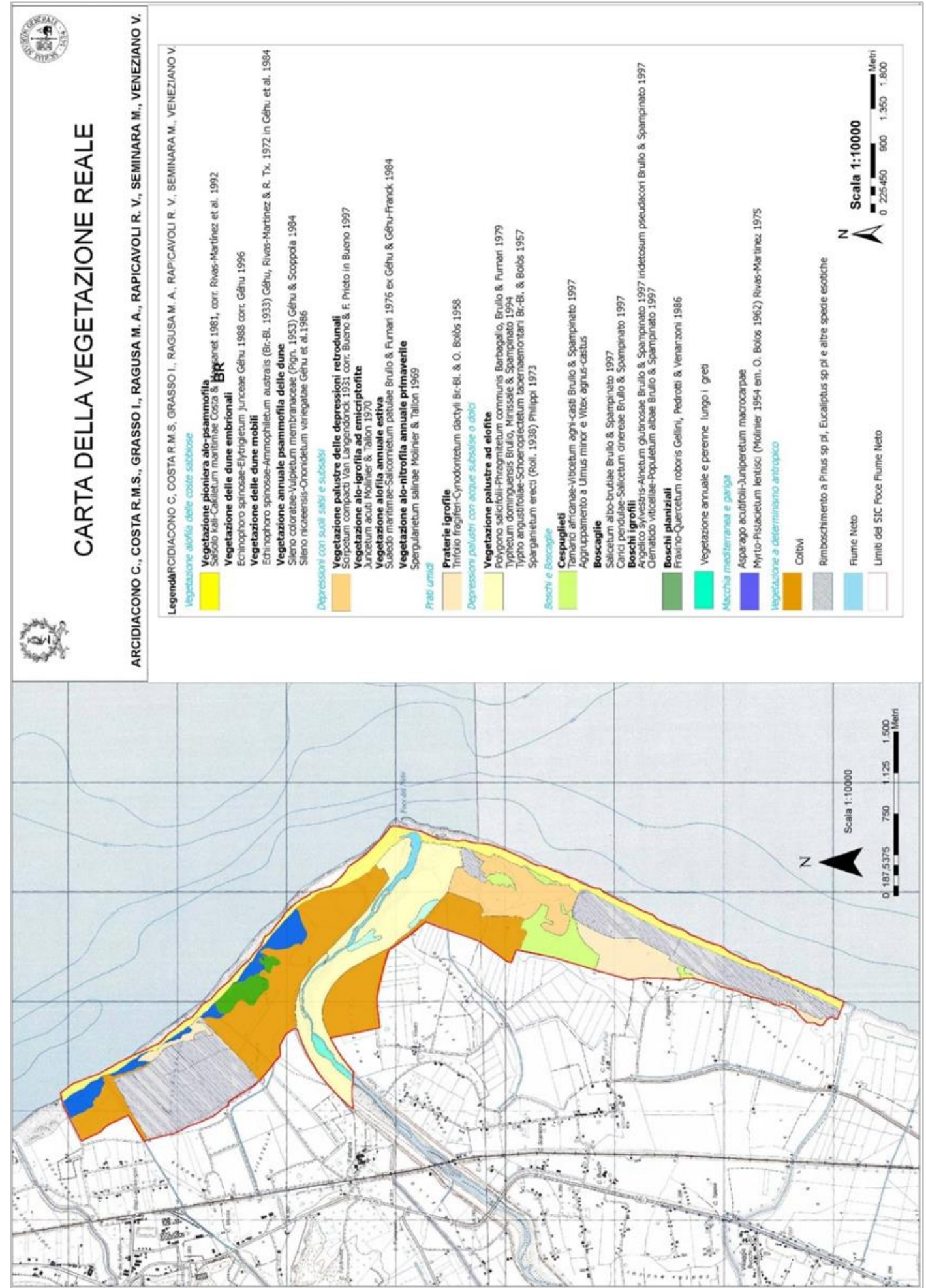




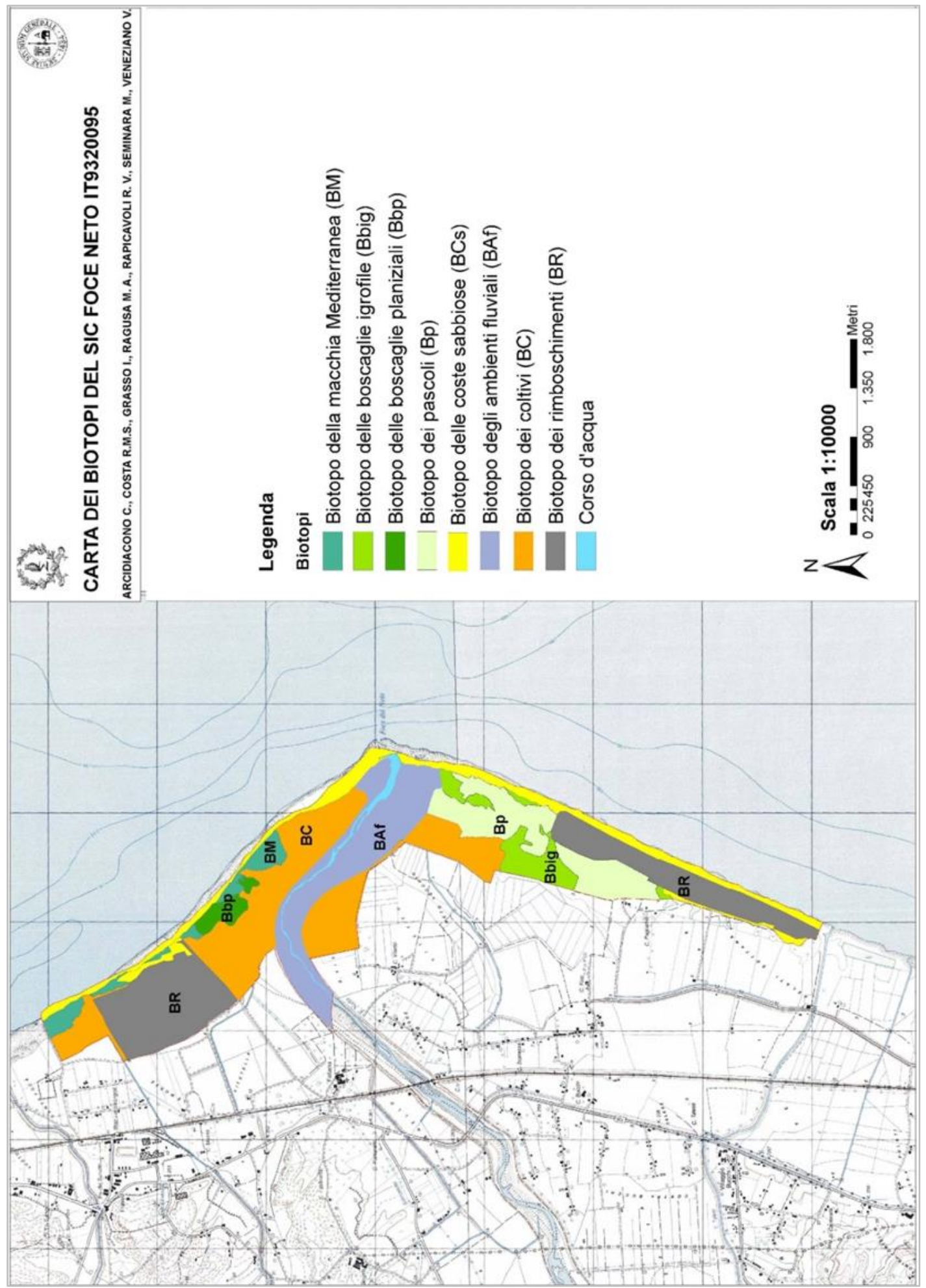




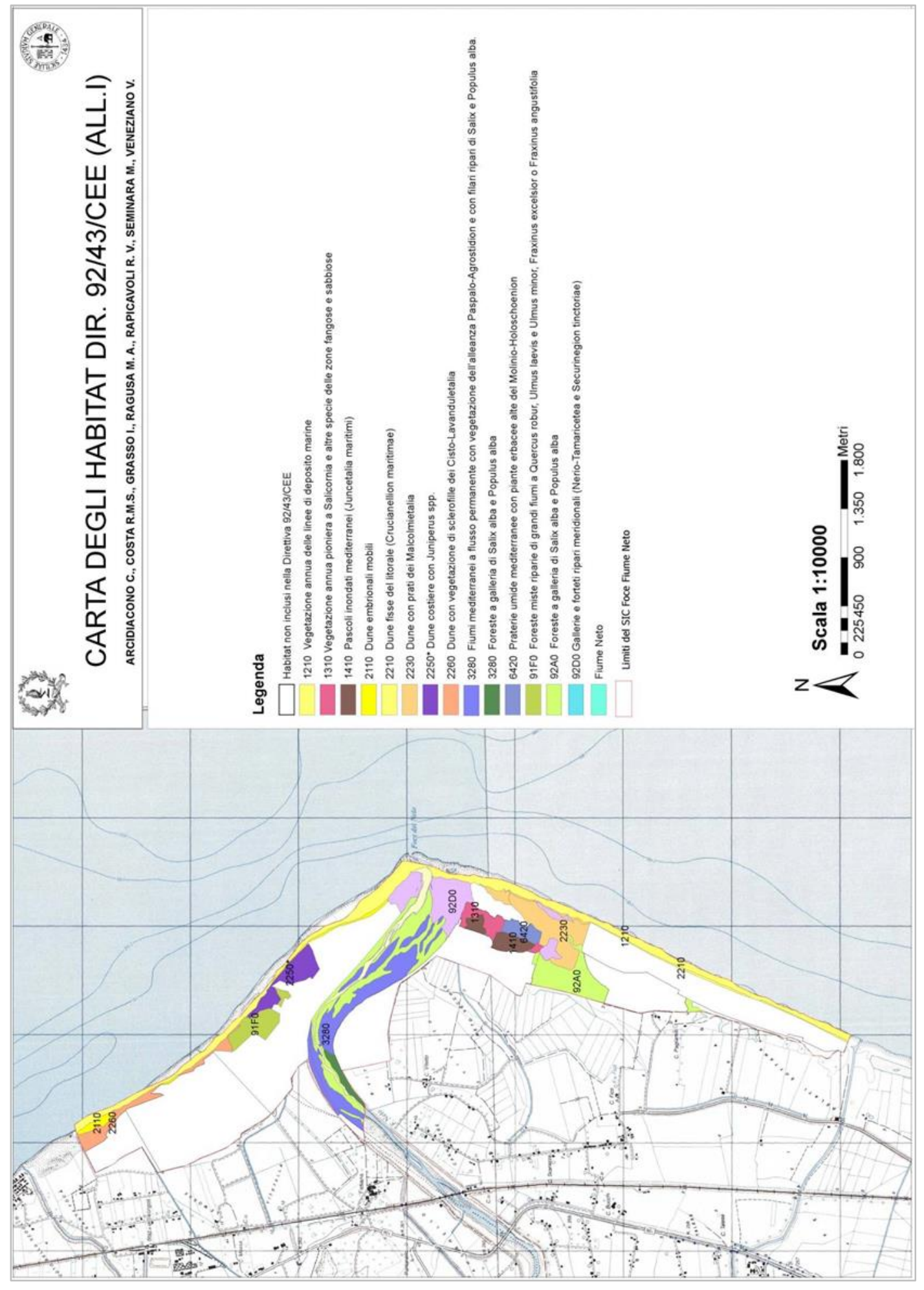




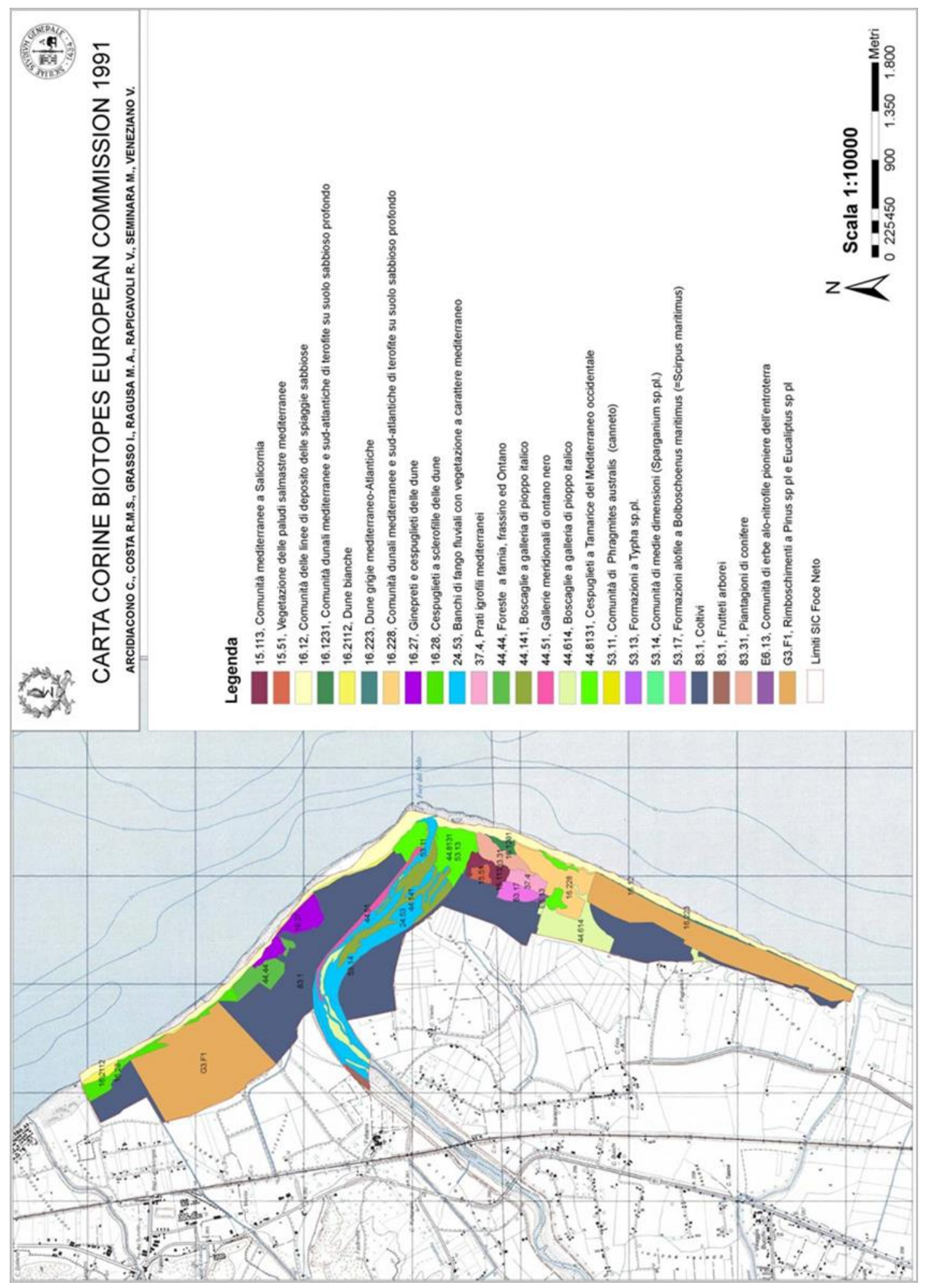




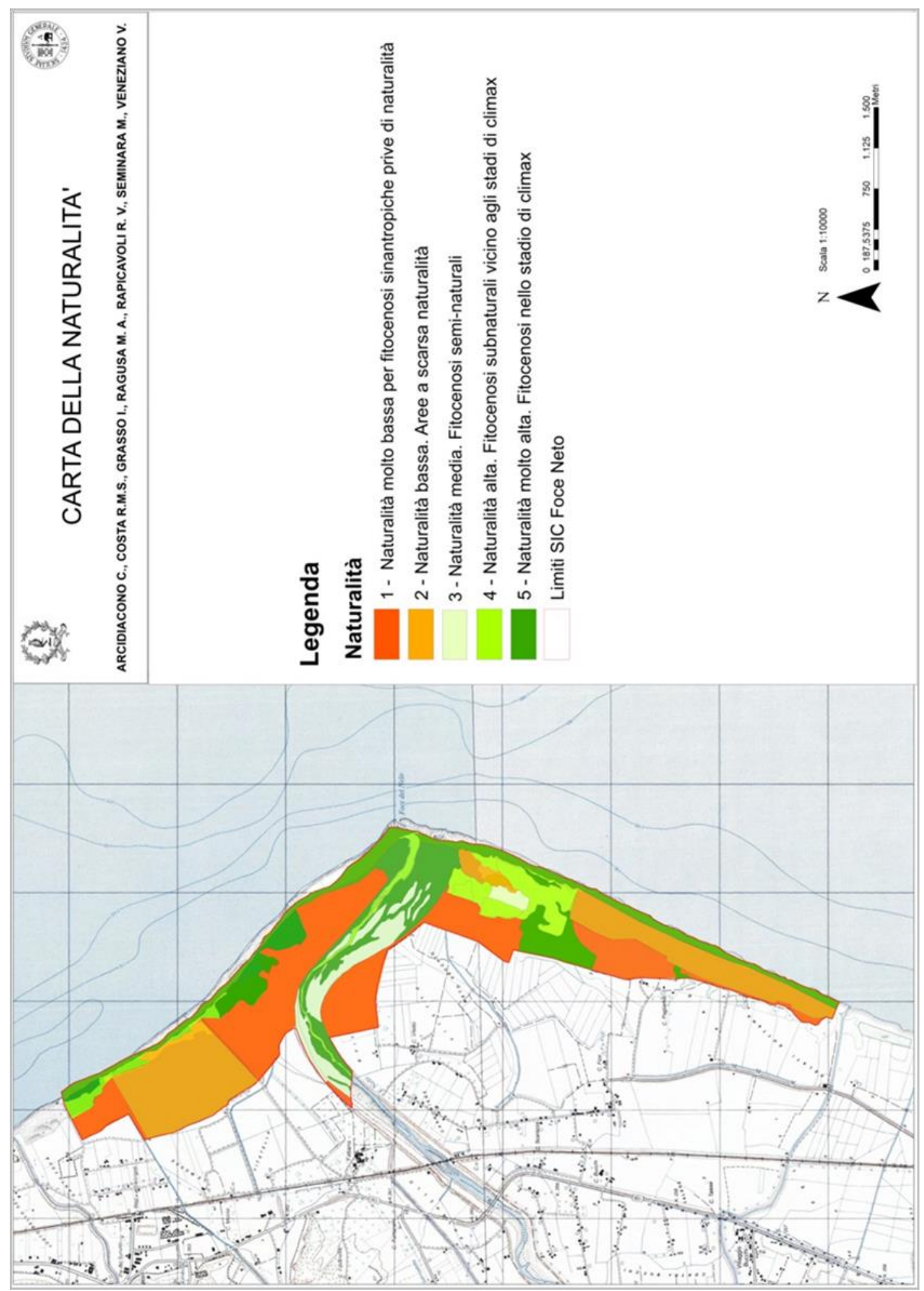




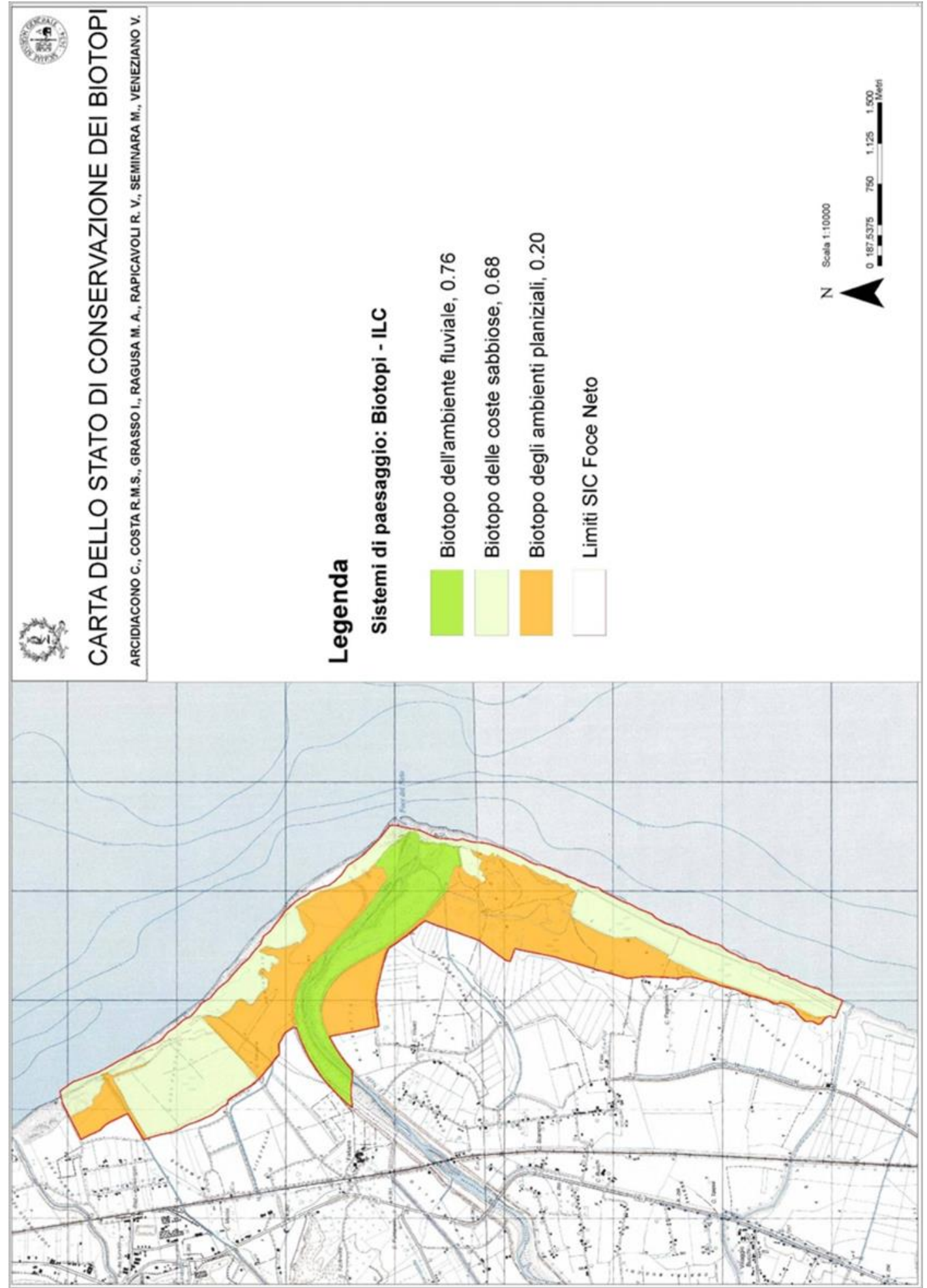




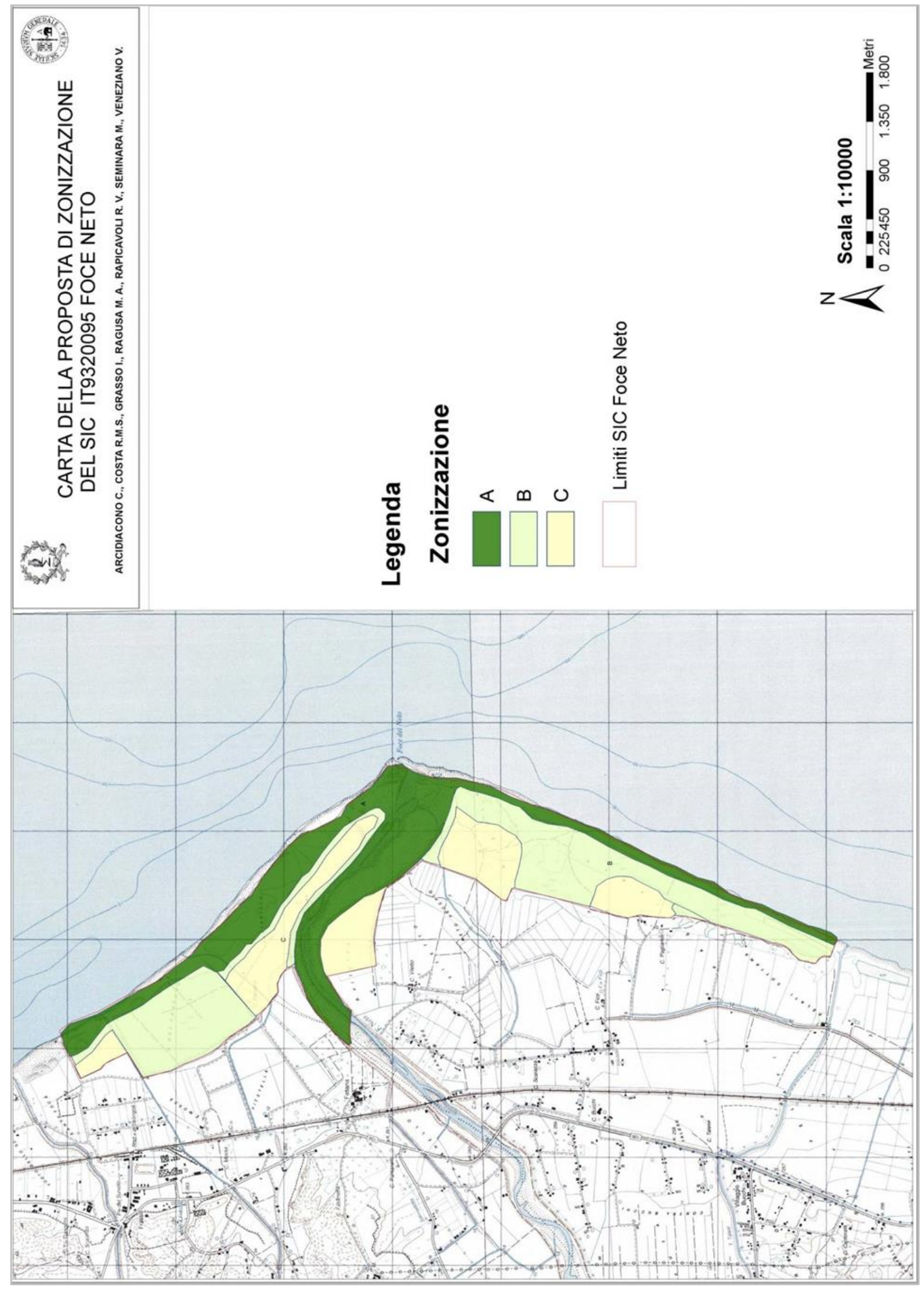




\subsection{Discussione}

Le analisi condotte hanno evidenziato uno stato di consevazione critico degli habitat costieri caratterizzati nello stesso tempo da un elevato valore e potenzialità nauturalistica. Infatti, la vegetazione naturale dell'area oggetto di studio si presenta rada in conseguenza di una gestione del territorio legata a opere di bonifica e rimboschimenti condotti sin dagli anni '50 per lo sfruttamento a fini agricoli del territorio. Le attività antropiche hanno favorito l'instaurarsi di diverse forme di degradazione della vegetazione originaria, rappresentata oggi prevalentemente da pascoli, praticelli effimeri, lembi residuali di boscaglie igrofile e localizzate. I residui di macchia a ginepro, di macchia a lentisco e il boschetto costiero a Farnia e Frassino sono presenti in maniera molto frammentata in sinistra idrografica rispetto al fiume Neto. Queste formazioni, individuate rispettivamente ai sensi della Direttiva 92/43CEE come habitat 2250*, 2260 e 91F0, sono di gran pregio e subiscono la pressione delle coltivazioni agricole, a ridosso e dal lato interno, e dell'erosione costiera dal fronte mare.

La biodiversità è stata valutata a livello di habitat e di paesaggio vegetale. Lo studio fitosociologico ha permesso di evidenziare aspetti vegetazionali riferibili al sistema dunale e retrodunale costiero, al sistema delle aree umide planiziali con boscaglie umide e pascoli alofili e subalofili, e a quello ripario con boscaglie a salici e pioppo bianco e vegetazione tipica dei corsi d'acqua.

Nel sito sono presenti due rimboschimenti di specie non autoctone per il territorio del genere Pinus sp pl ed Eucaliptus sp pl, realizzati intorno agli anni ' 50 e implementati negli anni '90 con ulteriori specie esotiche quali, Phoenix canariensis, Washingtonia filifera, Chamaerops humilis, Cupressus arizonica, Acacia saligna, Myoporum tenuifolium, Agave americana, ecc., soprattutto per la necessità di difendere $\mathrm{i}$ terreni coltivati retrostanti dai venti marini).

Questi impianti artificiali vengono considerati come habitat sensu Direttiva 92/43/CEE e riportati nella Scheda Natura 2000-standard data form del SIC Foce Neto, come habitat di tipo prioritario con il codice 2270*: Dune con foreste di Pinus pinea e/o Pinus pinaster. In prima istanza, l'interpretazione degli Habitat EUR 27 (2007) aveva escluso questo habitat per la Regione Calabria, perché comprendente "piantagioni mature di specie di pini, entro la loro naturale area di distribuzione e con sottobosco simile a quello delle formazioni paraclimaciche (Long-established plantations of these pines, within their natural area of occurrence, and with an undergrowth basically similar to that of paraclimacic formations, are included in this habitat type, Interpretation Manual of European Union Habitats, 2007).

Secondo la Direttiva Habitat, la combinazione fisionomica di riferimento dell'Habitat 2270* prevede la presenza di Pinus pinea, P. pinaster, P. halepensis, Juniperus oxycedrus ssp. macrocarpa, J. phoenicea ssp. turbinata.

Come si evince quindi dalla combinazione delle specie, l'habitat ha una sua composizione floristica caratteristica ed è identificato a livello europeo da formazioni a Pinus pinea, Pinus pinaster e Pinus halepensis, accompagnate da un abbondante sottobosco di specie della macchia mediterranea.

Le pinete artificiali comprese all'interno del SIC sono costituite da tre specie di pino, Pinus pinea, Pinus pinaster e Pinus halepensis. Il Pinus pinaster non è una specie autoctona dell'Italia meridionale (Pignatti, 1982; Jalas et Suominem, 1973); il Pinus pinea è coltivato ovunque in Italia ma è di dubbio indigenato per la Calabria così come lo è quello di Pinus halepensis (Pignatti, 1982).

Dai rilevamenti fitosociologici, effettuati all'interno del rimboschimento, è emerso che la pineta, essendo artificiale, presenta un evidente sesto d'impianto, con piante coetanee alte $5 \mathrm{~m}$ circa che 
conferiscono una struttura monoplana alla pineta. Il corteggio floristico non presenta aspetti della macchia ma è estremamente impoverito e con rada vegetazione erbacea annuale di tipo ruderale e senza alcun valore fitogeografico con elementi dei Malcolmietalia e dell'Alkanno-Malcolmion ramosissimae, quali Bromus rigidus, Cutandia maritima, Lagurus ovatus, pseudorlaya pumila, Silene nicaeensis. Inoltre, al margine della formazione sono presenti specie alloctone invasive come Opuntia sp. e Agave americana).

Muovendo dalle premesse legate alla definizione dell'habitat $2270^{*}$ e da quanto riportato sul Manuale d'interpretazione EUR 27 (2007) da una parte, e considerando la distribuzione delle specie di pino nell'Italia meridionale, il corteggio floristico rilevato con metodo fitosociologico e la struttura degli impianti dall'altra, discendono alcune considerazioni.

1) Nel caso specifico, sebbene la pineta sia diventata parte integrante del paesaggio costiero, non si può dimenticare che trattasi di una formazione vegetale a determinismo antropico impiantata sulle dune.

2) In conseguenza di ciò, è stata provocata una profonda alterazione della duna e della sua vegetazione naturale, poiché trattasi di una pineta che occupa la posizione del Crucianellion (habitat 2210 "Dune fisse del litorale del Crucianellion maritimae") e quella delle formazioni potenziali a Juniperus dell'habitat prioritario 2250* "Dune costiere con Juniperus spp.".

3) La presenza del rimboschimento, comporta una rinnovazione spontanea del pino e dell'eucalipto a scapito di emergenze floristiche localizzate nel retroduna come Artemisia variabilis Ten., endemica dell'Italia meridionale, Pancratium maritimum L., specie riportata per la Calabria nelle liste Rosse Regionali (Conti et al., 1997) come minacciata (EN) e con frequenza nell'area di studio molto rara, Ephedra distachia specie rara, inserita con il grado di minaccia a minor rischio (LR) nelle liste Rosse Regionali e Cyperus capitatus Vand specie con frequenza rara.

4) Irreversibili effetti sulle formazioni a Ginepro. Gli effetti negativi sulle formazioni di Ginepro sono documentate anche in Management of Natura 2000 Habitats 2250* Coastal dunes with Juniperus spp. (Picchi S., 2008). La presenza della pineta, oltre a comportare la diffusione di specie aliene e ruderali, mitigando l'effetto dell'areosol marino, causano la scomparsa delle formazioni endemiche di ginepro, promuovendo l'ingresso di specie terrestri ad ampia distribuzione, causando importanti cambiamenti nel corteggio floristico a causa di una diversa stabilizzazione delle dune. Le specie adattate a substrati sciolti scompaiono e la nicchia ecologica è invasa da specie meno tolleranti allo stress costiero. In queste condizioni, i ginepri sono meno competitivi, la deposizione degli aghi di pino impedisce alle giovani piantine di Juniperus, Myrtus, Pistacia, Phyllirea di stabilirsi e comporta la morte delle piante più mature.

Prendendo atto della documentazione ufficiale prodotta a livello europeo e le caratteristiche fitosociologiche ed ecologiche della piantaggione in oggetto, e cioè la mancanza di uno strato di macchia sotto la piantaggione artificiale di pino causata dai motivi di cui sopra, emerge la perplessità sulla corretta attribuzione di questa specifica pineta all'habitat di Direttiva 2270*, così come invece appare riportata nella Scheda Natura 2000 del SIC Foce Neto. Inoltre, si pone l'esigenza di controllare l'invasione e l'evoluzione delle neoformazioni di Pinus. 


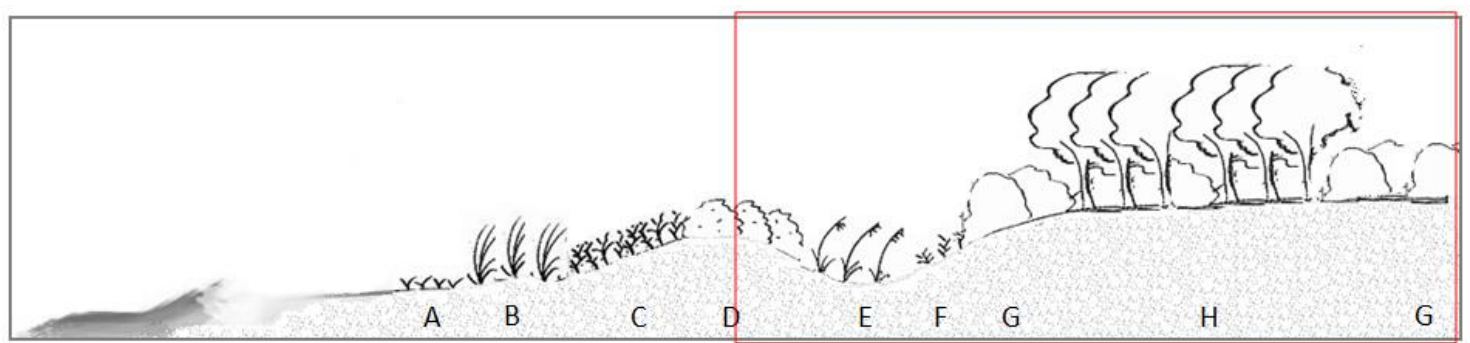

A Cakiletea, B Ammophiletea, C Crucianelletea, D Juniperion turbinatae, E Juncetea maritimi, F Malcolmietalia, $G$ Oleo-Ceratonion, $\boldsymbol{H}$ Foresta decidua. Transetto della vegetazione rilevato. All'interno del riquadro rosso ricadono gli aspetti vegetazionali scomparsi a causa della presenza del rimboschimento a Pinus sp pl. (da protocolli di monitoraggio degli habitat d'interesse comunitario nella rete natura 2000 in Calabria, modif.)

Le indagini fitosociologiche hanno permesso di individuare aspetti vegetazionali riconducibili a due macrocategorie: vegetazione naturale $(44.3 \%)$ e vegetazione a determinismo antropogeno (55.7\%). La vegetazione potenziale coerente con le caratteristiche bioclimatiche è rappresentata da fitocenosi di tipo arbustivo o arbustivo-boschivo di tipo termofilo ascrivibili all'OleoLentiscetum e Pistacio-Quercetum ilicis. Le formazioni di tipo azonale sono fortemente influenzate dai parametri edafo-igrofici, dal livello di salinità, dalla distanza dal mare e dal corso d'acqua; parametri ecologici che possono variare repentinamente. Dal punto di vista conservazionistico, il punto di fragilità del sistema è rappresentato dall'Unità paesaggistica degli ambienti planiziali in cui insistono le attività agricole. Quest'Unità paesaggistica andrebbe riqualificata attraverso un insieme di interventi volti al recupero di condizioni di maggior naturalità e alla riduzione della frammentazione degli habitat naturali (es. favorire l'evoluzione delle formazioni preforestali coerenti con la vegetazione del bosco edafoigrofilo, prevedere l'impianto di nuovi arbusteti al fine di implementare la connettività e funzionalità della rete ecologica territoriale, ecc.). Inoltre, lo stesso paesaggio agricolo andrebbe valorizzato attraverso azioni volte, per esempio, alla reintroduzione all'interno della matrice agricola di elementi di naturalità lungo i confini delle proprietà, (interventi lineari, piccoli nuclei di boschi e arbusteti) o sulle superfici agricole non utilizzate.

Acquisire un'adeguata conoscenza del patrimonio vegetale per mezzo di indagini sulla vegetazione è condizione indispensabile per orientare una gestione del territorio consapevole.

Le indagini sulla vegetazione sono necessarie per adeguare le esigenze di conservazione della biodiversità, in accordo con le Direttive Comunitarie 79/409 e 92/43, con le tradizionali pratiche agricole.

\section{Bibliografia}

AA.VV., 1991 - CORINE Biotopes manual. Commission of the European Communities, Brussel.

Angelini P., Casella L., Grignetti A. \& Genovesi P. (ed.), 2016 - Manuali per il monitoraggio di specie e habitat di interesse comunitario (Direttiva 92/43/CEE) in Italia: habitat. ISPRA, Serie Manuali e linee guida, 142/2016.

ARSSA, 2003. I suoli della Calabria. Carta dei suoli in scala 1:250.000 della Regione Calabria. Monografia divulgativa. ARSSA. 
Barbati, A. \& Chirici, G., 2009 - Analisi della struttura spaziale e pianificazione del paesaggio agro-forestale: prospettive d'integrazione. In: Ciancio, O. (a cura di). Atti del Terzo Congresso Nazionale di Selvicoltura (Taormina, 16-19 Ottobre 2008). Firenze, Accademia Italiana di Scienze Forestali, pp. 954-959.

Barbera B., Niccoli R. \& Piccione V., (a cura di), 2005 - Carta delle Aree Sensibili alla Desertificazione in Calabria. Pubblicazione Calabria. - Rubbettino Editore, 60.

Batzella C., Balvis T., Muntoni F. \& Marini A., 2012 - Carta della vegetazione e valutazione della naturalità del territorio dell'Ogliastra (provincia di Cagliari, Italy). Forest@ 9: 130-136 [ online 2012-06-26] URL: http://www.sisef.it/ forest@/contents/?id=efor0671-008

Biondi E., \& Baldoni M., 1995 - The climate and vegetation of peninsular Italy. Colloques Phytosociologiques, 23, 675-721.

Biondi E., Blasi C., Burrascano S., Casavecchia S., Copiz R., Del Vico E., Galdenzi D., Gigante D., Lasen C., Spampinato G., Venanzoni R. \& Zivkovic L., 2009 - Italian interpretation manual of the 92/43/EEC Directive Habitats. Available on-line at: http://vnr.unipg.it/habitat/index.jsp

Biondi E. \& Colosi L., 2005a - Environmental quality: an assessment based on the characters of the plant landscape. Plant Biosystem 139 (2): 145-154.

Biondi E. \& Nanni L., 2005b - Geosigmeti, unità di paesaggio e reti ecologiche. In: Carlo Blasi, Adriano Paolella (a cura) Identificazione e cambiamenti nel paesaggio contemporaneo. Atti del Terzo Congresso IAED (Roma, 4/6 dicembre 2003): 134-140.

Braun-Blanquet J., 1964 - Pflanzensoziologie-Grundzüge der Vegetations kunde. Vienna: Springer-Verlag, $865 \mathrm{pp}$.

Brullo S., De Santis C., Furnari F., Longhitano N. \& Ronsisvalle G.A., 1988 - La vegetazione dell'Oasi della Foce del Simeto (Sicilia orientale). Braun-Blanquetia 2:165-188.

Brullo S., Giusso Del Galdo G., Siracusa G. \& Spampinato G., 2002 - Considerazioni fitogeografiche sulla vegetazione psammofila dei litorali italiani. Biogeographia 22: 93-137.

Brullo S., Scelsi F.\& Spampinato G., 2001 - La Vegetazione dell'Aspromonte. Studio fitosociologico. Laruffa editore, Reggio Calabria.

Brullo S. \& Spampinato G., 1990 - La vegetazione dei corsi d'acqua della Sicilia. Boll. Acc. Gioenia Sci. Nat., 23 (336): 119-252.

Brullo S. \& Spampinato G., 1997 - Indagine fitosociologica sulle ripisilve della Calabria (Italia meridionale). Lazaroa 18: 105-151.

Caridi D., Maiorca G., Spampinato G., Cameriere P. \& Crisafulli A., 2006 - Analisi diacronica della vegetazione della riserva "Foce del Fiume Crati" (Calabria, Italia). Atti $10^{\circ}$ Conferenza ASITA. Bolzano, 14-17 novembre 2006. 
CCN-Comitato Capitale Naturale, 2017 - Primo Rapporto sullo Stato del Capitale Naturale in Italia. Disponibile al link: http://www.minambiente.it/ sites/default/files/archivio/allegati/ sviluppo_sostenibile/rapporto_capitale_naturale_Italia_17052017.pdf

Celesti-Grapow L., Alessandrini A., Arrigoni P.V., Banfi E., Bernardo L., Bovio M., Brundu G., Cagiotti M.R., Camarda I., Carli E., Conti F., Fascetti S., Galasso G., Gubellini L., La Valva V., Lucchese F., Marchiori S., Mazzola P., Peccenini S., Poldini L., Pretto F., Prosser F., Siniscalco C., Villani M. C., Viegi L., Wilhalm T. \& Blasi C. (Eds.), 2009a - Inventory of the non-native flora of Italy. Plant Biosystem, 143 (2):386-430.

Celesti-Grapow L., Pretto F., Carli E. \& Blasi C. (Eds.), 2009b - Non-native flora of Italy. Plant invasion in Italy - an overview (CD-ROM). Roma.

Cerabolini B., Raimondi B., Cattaneo M., Preatoni D. \& Brusa G., 2007 - I caratteri della vegetazione come descrittori della qualità ambientale: un'applicazione cartografica (provincia di Varese, Lombardia). Informatore Botanico Italiano 39: 155-165.

Conti F., Manzi A. \& Pedrotti F., 1997 - Liste Rosse Regionali delle Piante d'Italia. WWF Italia - Società Botanica Italiana.

Conti F., Abbate G., Alessandrini A. \& Blasi C., 2005 - Anannotated checklist of the Italian vascular flora. Ed. Palombi, Roma-

Edarra, 1997 - Botanica ambiental aplicada. Pamplona: Eunsa.

Euro+Med, 2006-2018 - Euro+Med PlantBase - the information resource for EuroMediterranean plant diversity [http://ww2.bgbm.org/EuroPlusMed/].

Ferrari C., Pezzi G. \& Dell'Aquila L. 2000 - Diversità e naturalità della vegetazione. Elementi per un'analisi quantitativa integrata. Informatore Botanico Italiano 32 (1): 31-34.

Ferrari C., G. Pezzi, L. Diani \& Corazza M., 2008 - Evaluating landscape quality with vegetation naturalness maps: an index and some inferences. Applied Vegetation Science Apr 2008: Vol. 11, Issue 2, pg (s) 243- 250 https://doi.org/ 10.3170/2008-7-18400

Forman R.T.T., 1986 - Landscape ecology. John Wiley and Sons.

Frondoni R. \& Iberite M., 2002 - The alophile vegetation of the sedimentary coast of Lazio (Central Tyrrhenian district, Italy). Plant Biosystems 136(1): 49-68.

Greuter W., Burdet H.M. \& Long G. (eds.), 1984-1989 - Med-Checklist 1, 3, 4. Conservatoire et Jardin botaniques, Genève.

Jalas J. \& Suominen J., 1973 - Atlas Florae Europaeae 2. Committee for Mapping the Flora of Europe and Societats Biologica Fennica Vanamo, Helsinki. 
Kosmas C., Kirkby M. \& Geeson N., 1999 - The Medalus project. Mediterranean desertification and land use. Manual on key indicators of desertification and mapping environmentally sensitive areas to desertification. Bruxelles, Belgium. EUR 18882,88.

ISPRA, 2009 - Il progetto Carta della Natura Linee guida per la cartografia e la valutazione degli habitat alla scala 1:50.000. A cura del Dipartimento Difesa della Natura - ISPRA Servizio Carta della Natura. Roma.

ISTAT, $2013-6^{\circ}$ Censimento Generale dell'Agricoltura. Roma.

Lausi D., Pignatti S. \& Poldini L., 1978 - Carta della vegetazione dell'alto Friuli - Zona colpita dai terremoti del maggio-settembre 1976. Collana del P.F.Promozione della Qualità dell' Ambiente. CNR, AQ - 1.3.

Maiorca G. \& Spampinato G., 1999 - La vegetazione della Riserva Naturale Orientata "Valle del Fiume Argentino" (Calabria Nord-Occidentale). Fitosociologia 36 (2): 15-60.

Maiorca G., Spampinato G. \& Caprio A., 2002 - Flora e vegetazione dei laghi costieri La Vota (Calabria Centro-Occidentale). Fitosociologia, 39 (1): 84-105.

Maiorca G., Spampinato G., Crisafulli A. \& Cameriere P., 2007 - Flora vascolare e vegetazione della Riserva Naturale Regionale "Foce del Fiume Crati" (Calabria, Italia meridionale). Webbia, 62 (2): 121-174.

MATTM, 2010. Manuale di interpretazione degli habitat. Roma.

Maiorca G., Spampinato G., Cameriere P., Crisafulli A., Caridi D., Paleologo P. \& Grasso S., 2005 - Carta della Vegetazione Reale della Foce del Fiume Crati (CS - Calabria) (scala 1:4000) - Progetto Phytosis (Arssa - Università Mediterranea di Reggio Calabria), Monografia n.2, pp. 64. Ed. De Rose. Cosenza.

Micciardi M.R. \& Gargini V., 2003 - La valutazione della naturalità e della vulnerabilità di un territorio. In Casagrandi R., Melià P. (eds), Ecologia Quantitativa: metodi sperimentali, modelli teorici, applicazioni. Aracne, Roma, pp. 130-136.

MINISTERO LL.PP. - Servizio Idrografico, 1926-2000 - Annali Idrologici. Parte I. Roma.

Moss D. \& Davies C., 1999 - Eunis habitat classifications. United Kingdom.

Picchi S., 2008 - Management of Natura 2000 habitats. 2250* Coastal dunes with Juniperus spp. European Commission.

Pignatti S., 1982 - Flora d'Italia. Bologna: Edagricole. pp. 1-3.

Pizzolotto R. \& Brandmayr P., 1996 - An index to evaluate landscape conservation state based on land-use pattern analysis and Geographic Information System techniques, in Coenoses, 11, 1996, pp. 37-44.

Poldini L. \& Pertot M., 1989 - Criteri di indicizzazione del valore naturalistico sull'esempio del Carso triestino - goriziano. Inform. Bot. Ital. 21 (1-3): 133-151. 
Rivas-Martínez S. \& Loidi Arregui J., 1999 - Bioclimatology of the Iberian Peninsula. Itinera Geobot. 13:41-47.

Spampinato G., Crisafulli A.\& Cameriere P., 2007 -Trasformazione delle zone costiere della Regione Calabria (Italia meridionale) e conseguenze sulla flora degli ambienti umidi. Fitosociologia, 44 (2) 119-128.

Spampinato G., Cameriere P., Caridi D. \& Crisafulli A., 2008 - Carta della biodiversità del Parco Nazionale dell'Aspromonte. Quad. Bot. Amb. Appl. 19: 3-36.

Tomasella M., Vidali M., Oriolo G., Poldini L., Comin S. \& Giorgi R., 2007 - Valutazione della qualità degli habitat della costa sedimentaria (Laguna di Marano e Grado) e della costa a falesie (Costiera triestina): applicazione del metodo EsAmbI. Fitosociologia 44 (1): 17-31

Tutin, T. G. et al. (eds.), 1964-1980 - Flora Europaea. Vols. 1-5. Cambridge University Press, Cambridge.

Villa F., 1995 - Linee guida perla rilevazione e la valutazione dei parametri ambientali richiesti dal progetto "Rete Natura 2000”. SITE Notizie 15 (1): 67-75.

Westhoff, V. \& E. van der Maarel., 1978 - The Braun-Blanquet approach. In Whittaker, R. H., editor. (ed.). Classification of plant communities. 2nd. Ed pp. 287-399. Junk. The Hague, NL.

\section{AUTORI}

Arcidiacono Claudia - Professore Ordinario di Costruzioni rurali e territorio agroforestale, Dipartimento di Agricoltura, Alimentazione e Ambiente, Università degli Studi di Catania

Costa Rosanna Maria Stefania - Già Docente di Botanica Ambientale Applicata Dipartimento di Scienze Biologiche, Geologiche, Ambientali, Sez. Botanica, Università degli Studi di Catania

Grasso Irene - Dottore Magistrale in Salvaguardia del Territorio, dell'Ambiente e del Paesaggio Università degli Studi di Catania

Ragusa Maria Alessandra - Prof. Ordinario di Analisi Matematica, Esperta in Modelli Matematici per l'Ambiente e Delegata del Rettore per la Internazionalizzazione ambito scientifico, Dipartimento di Matematica ed Informatica, Università degli Studi di Catania

Rapicavoli Rosaria Valentina - Biologa, Dottore Magistrale in Scienza della Tutela dell'Ambiente Università degli Studi di Catania

Seminara Michele - Informatico, Esperto in Data Base Università degli Studi di Catania

Veneziano Vincenzo - Biologo, Dottore di Ricerca (Ph.D.) in Biologia ed Ecologia Vegetale in Ambiente Mediterraneo Università degli Studi di Catania

\section{RINGRAZIAMENTI}

Si ringrazia il Prof. Francesco Furnari, già Professore Ordinario di Botanica e Direttore del Dipartimento di Botanica dell'Università degli Studi di Catania per la presentazione di questa ricerca nella qualità di Socio Emerito dell'Accademia Gioenia. 
Allegato I

CARTE DELL'USO DEL SUOLO

CLC IV LIVELLO 2012

IT9310042 Fiumara Saraceno

IT9310043 Fiumara Avena

IT9310044 Fiume Crati

IT9310047 Fiumara Trionto

IT9320095 Foce Neto

IT9330105 Foce del Crocchio - Cropani

IT9350132 Fiumara di Melito

IT9350145 Fiumara Amendolea

IT9350146 Fiumara Buonamico

IT9350147 Fiumara Laverde 


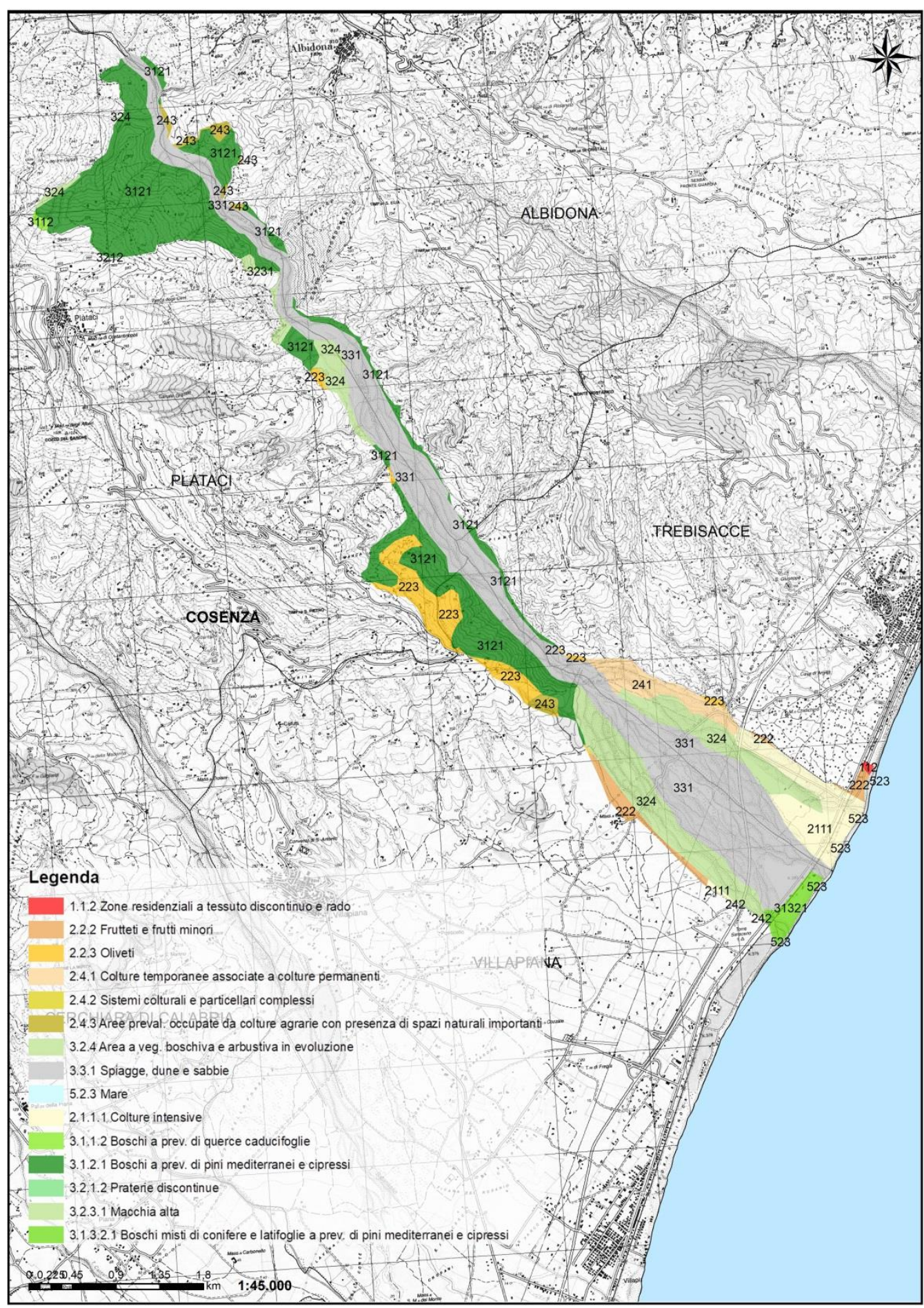



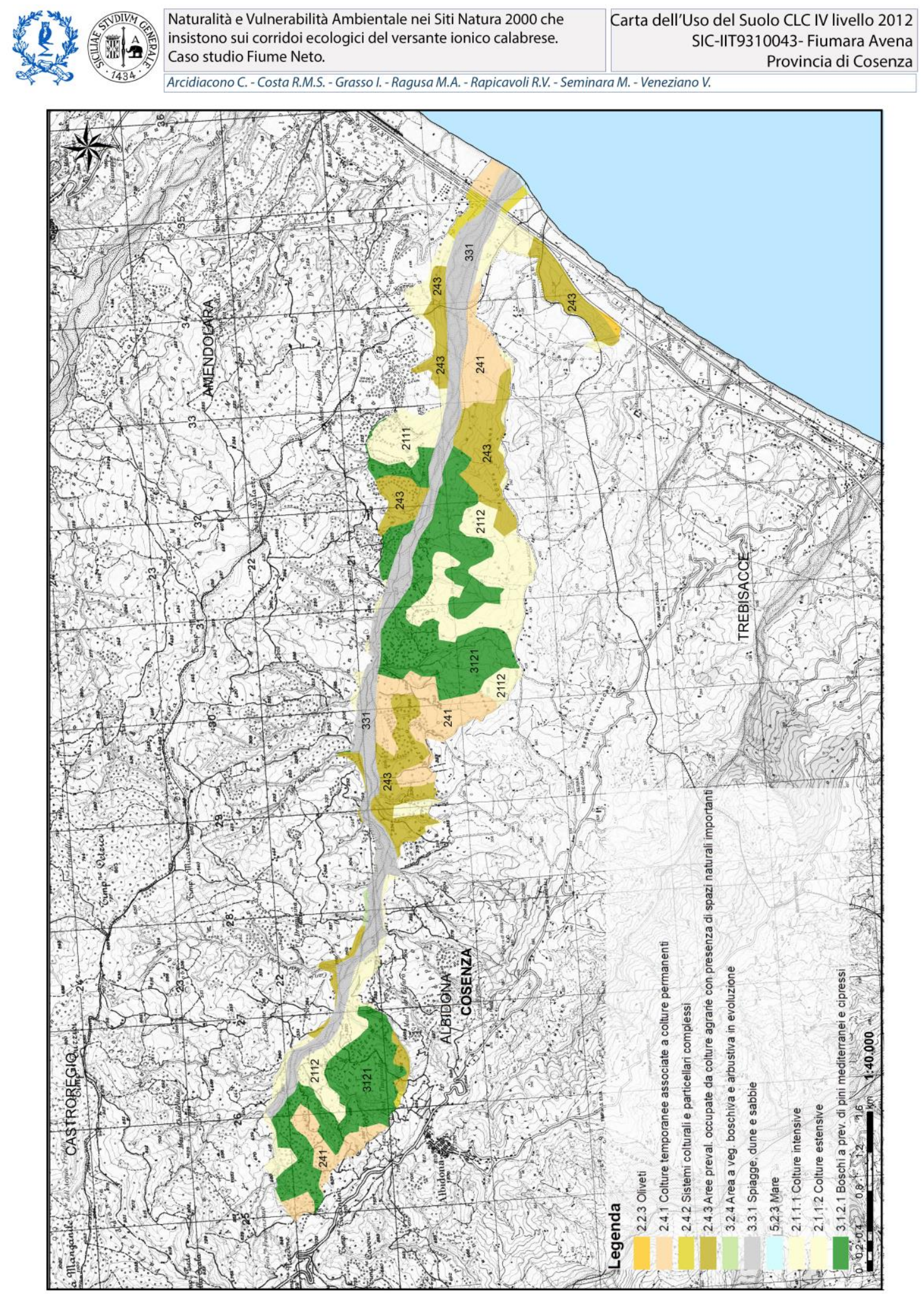
Arcidiacono C. - Costa R.M.S. - Grasso I. - Ragusa M.A. - Rapicavoli R.V. - Seminara M. - Veneziano V. Provincia di Cosenza

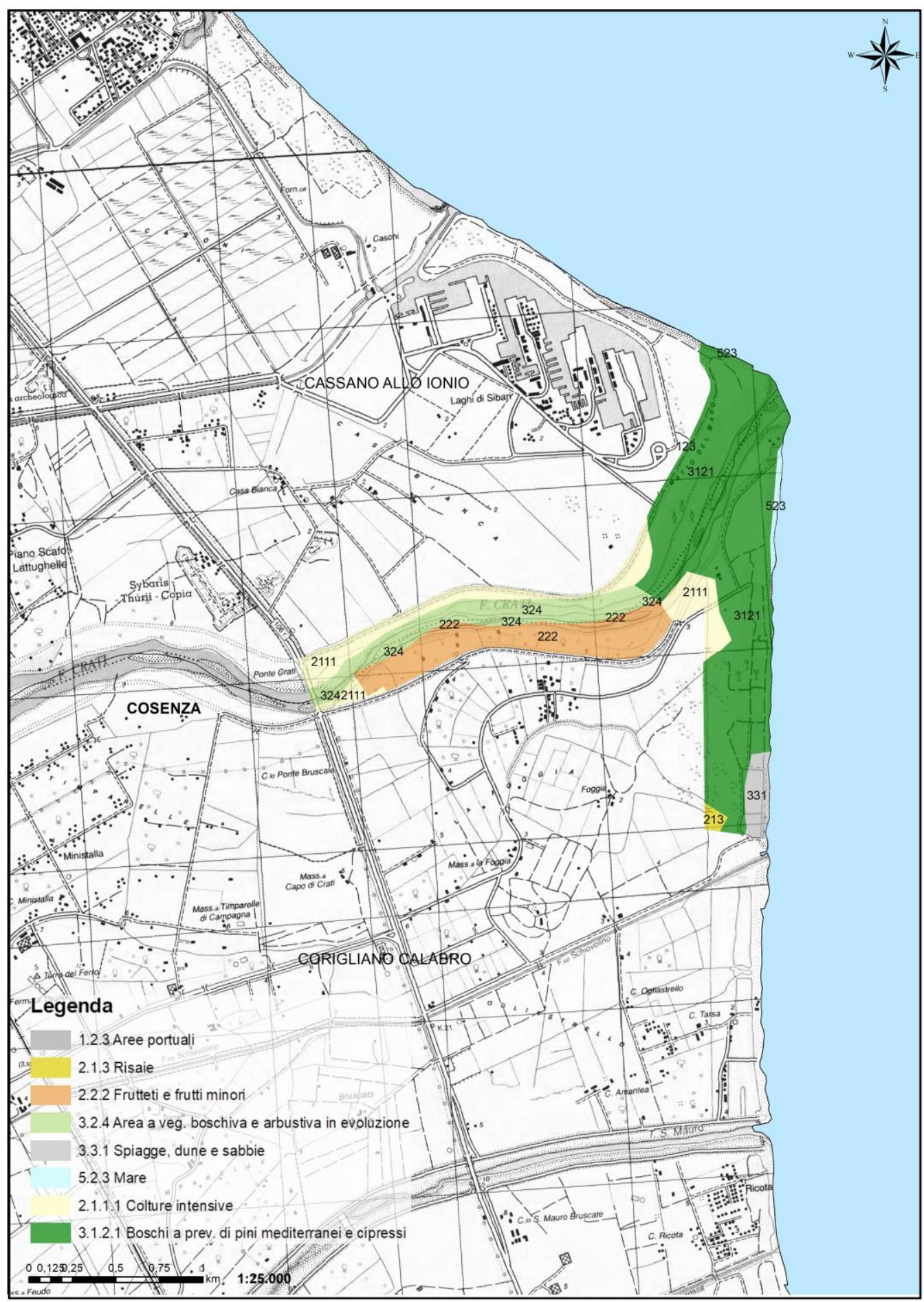



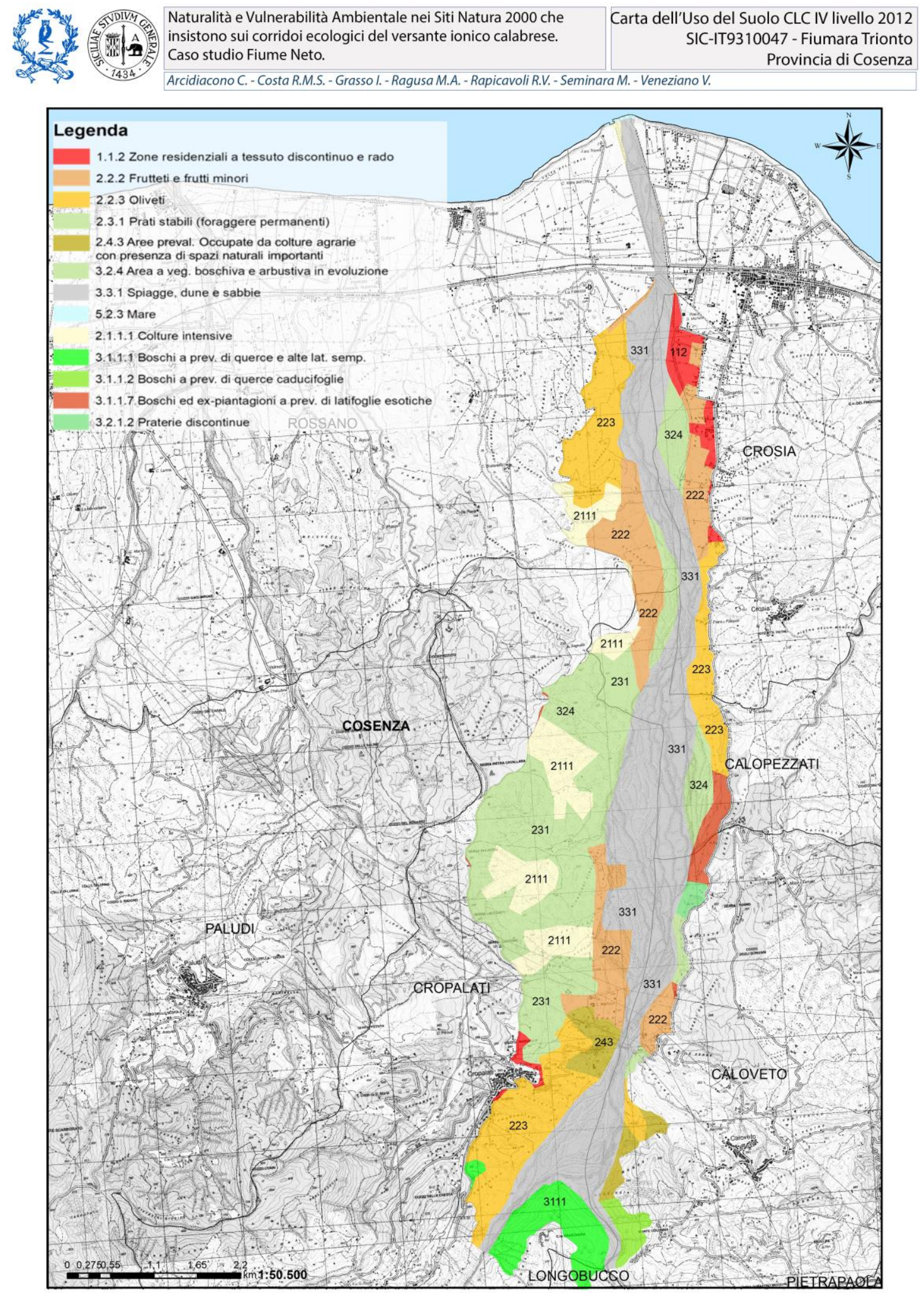

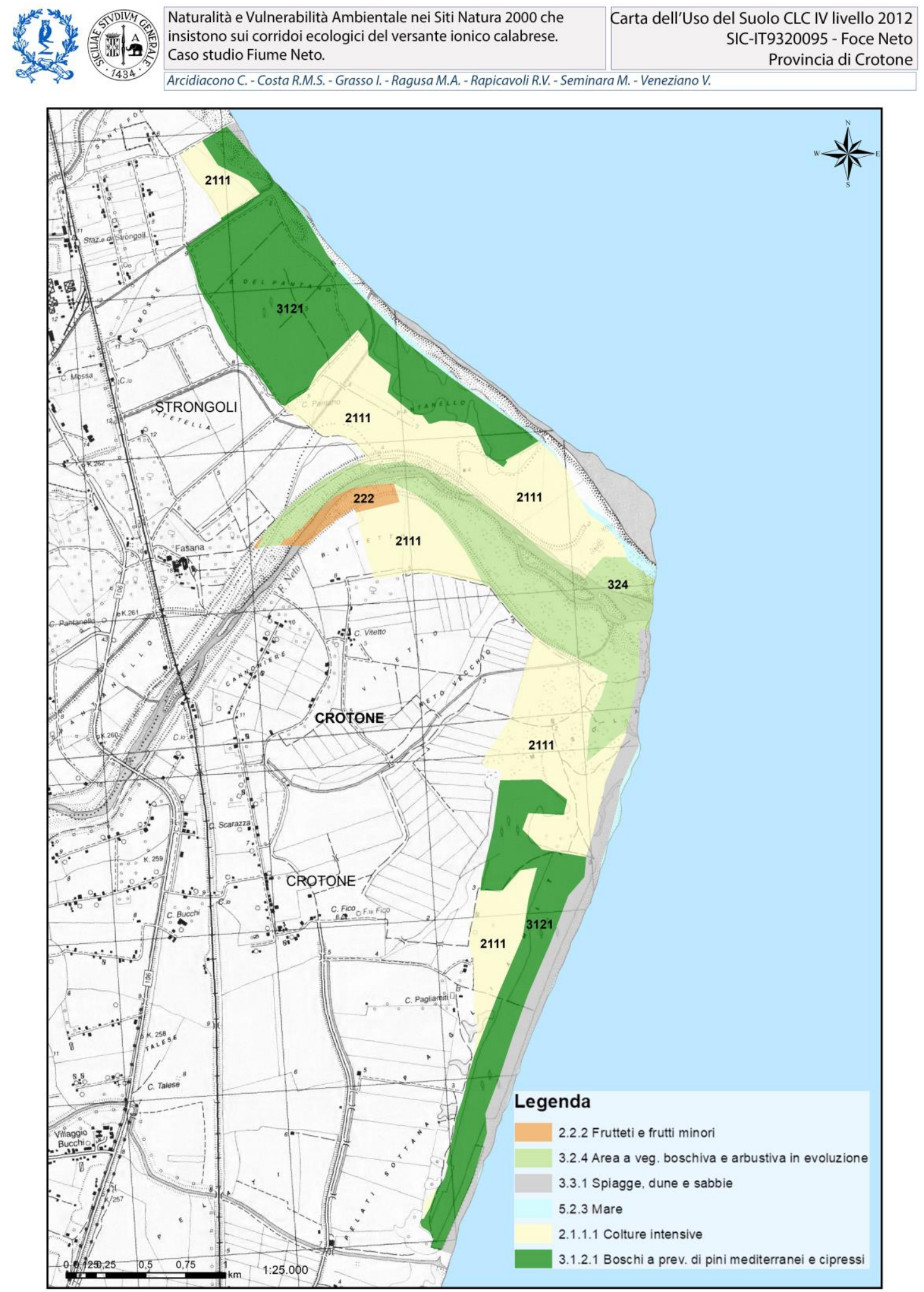
Arcidiacono C. - Costa R.M.S. - Grasso I. - Ragusa M.A. - Rapicavoli R.V. - Seminara M. - Veneziano V. Provincia di Catanzaro

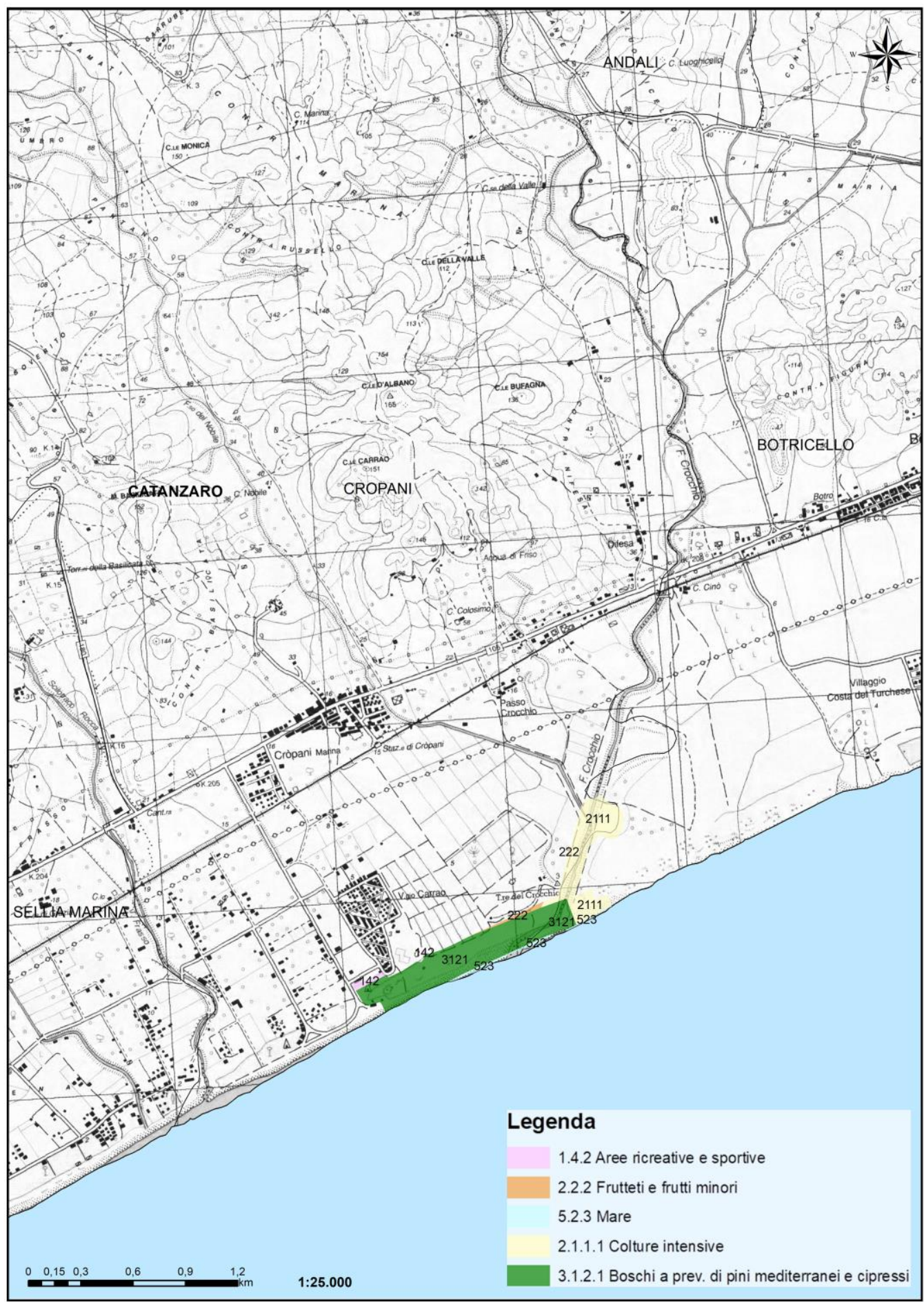



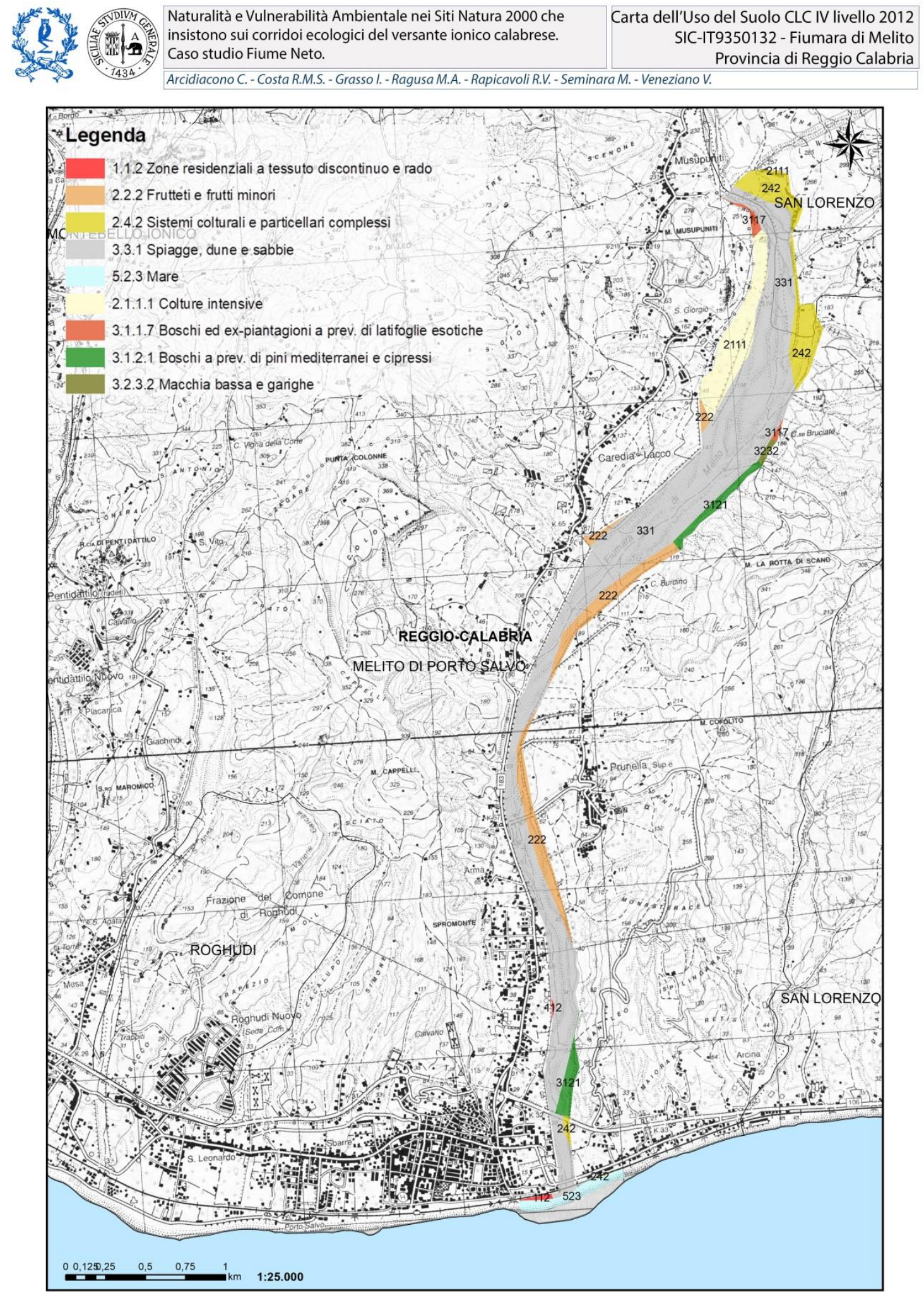


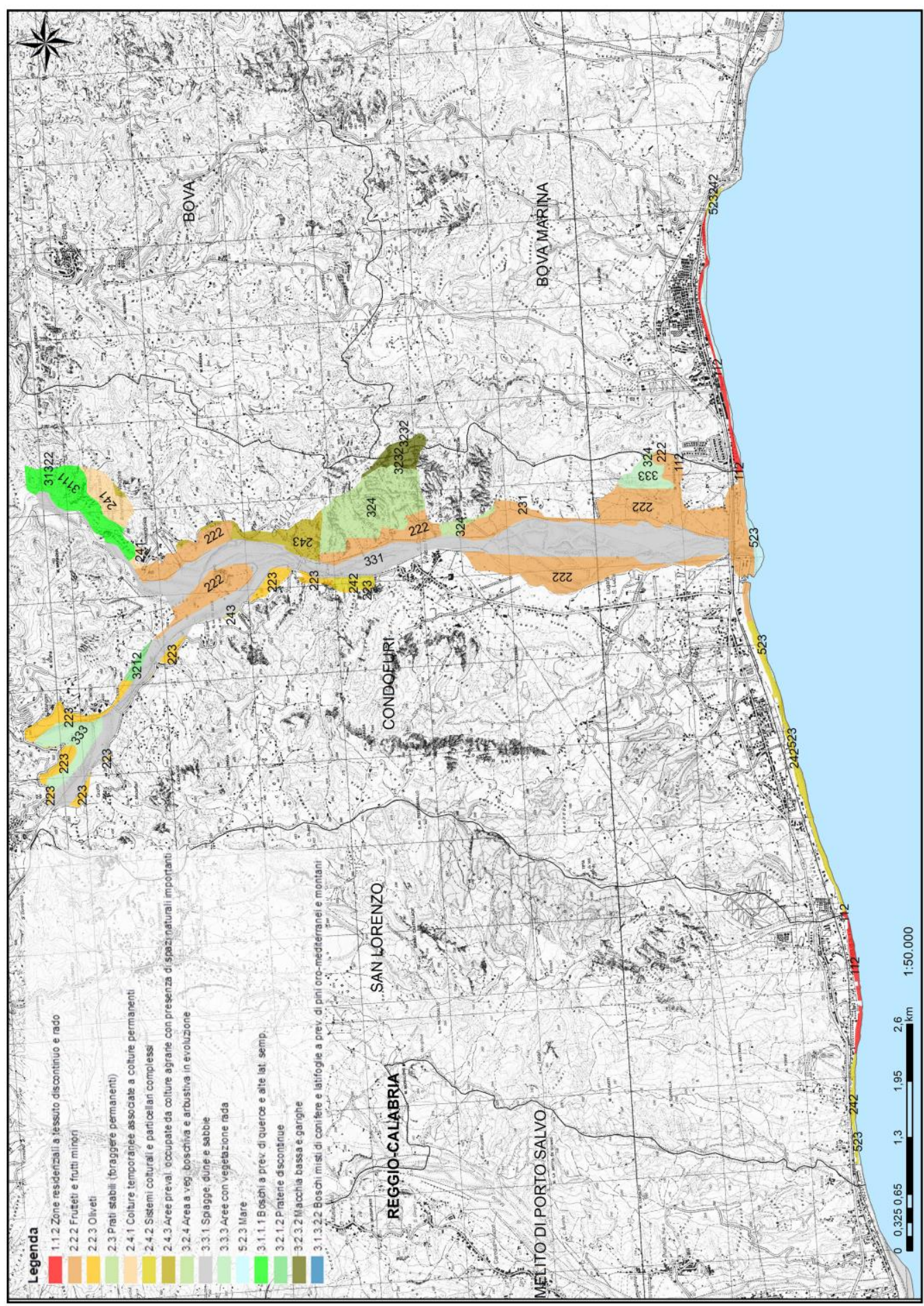



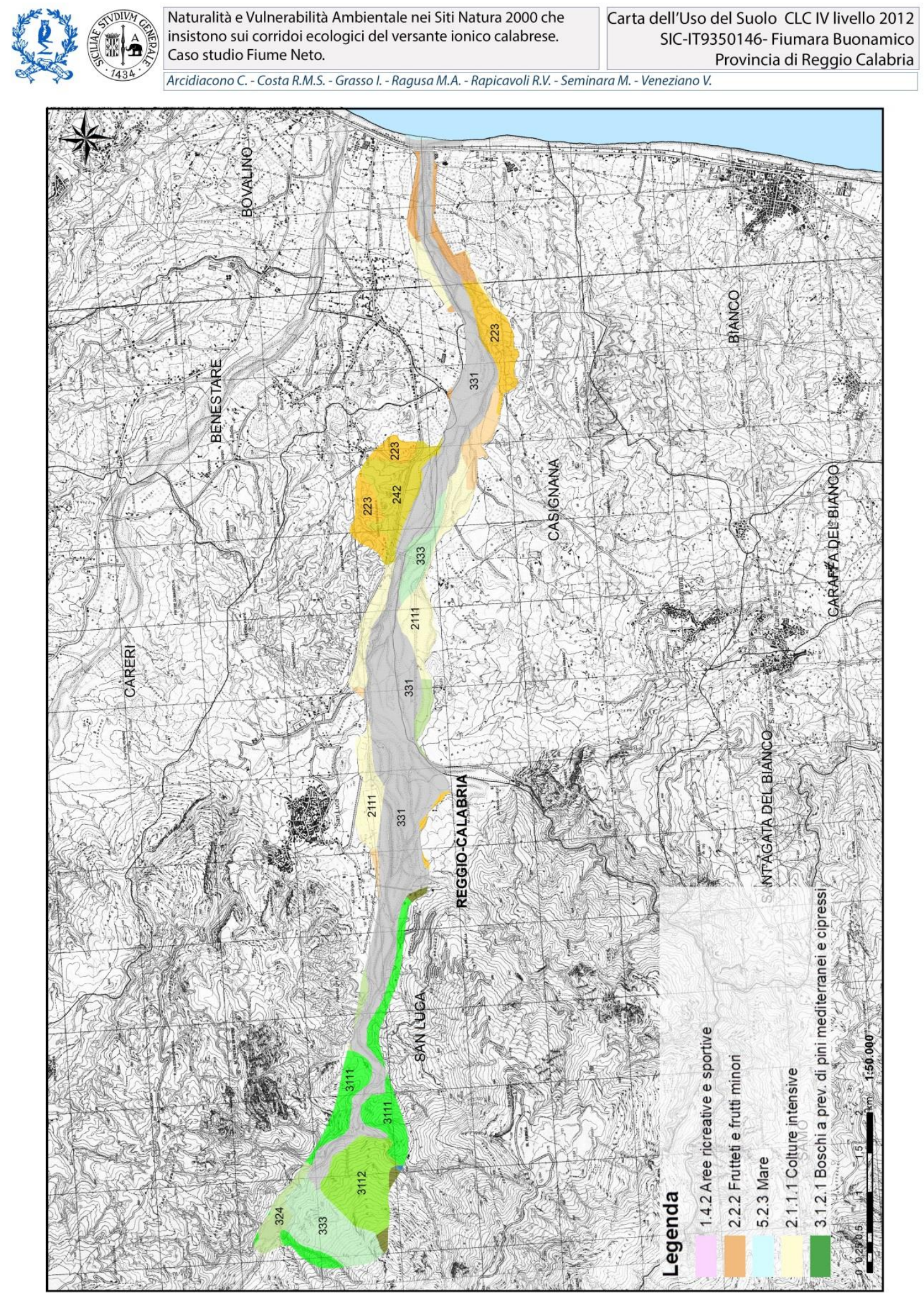

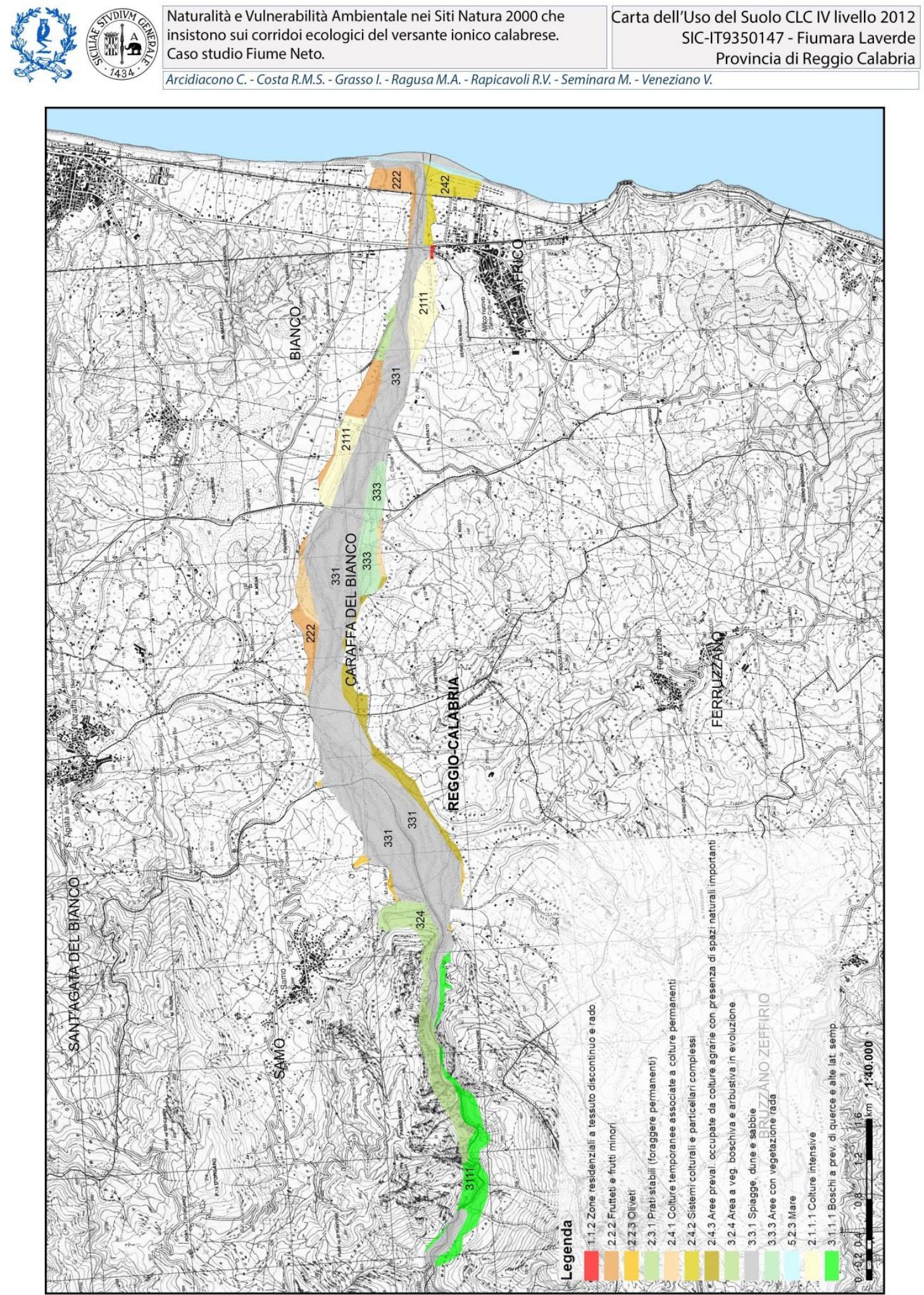
Allegato II

CARTE DELLA NATURALITÀ ARTIFICIALITÀ

IT9310042 Fiumara Saraceno

IT9310043 Fiumara Avena

IT9310044 Fiume Crati IT9310047 Fiumara Trionto

IT9320095 Foce Neto

IT9330105 Foce del Crocchio - Cropani IT9350132 Fiumara di Melito IT9350145 Fiumara Amendolea IT9350146 Fiumara Buonamico IT9350147 Fiumara Laverde 

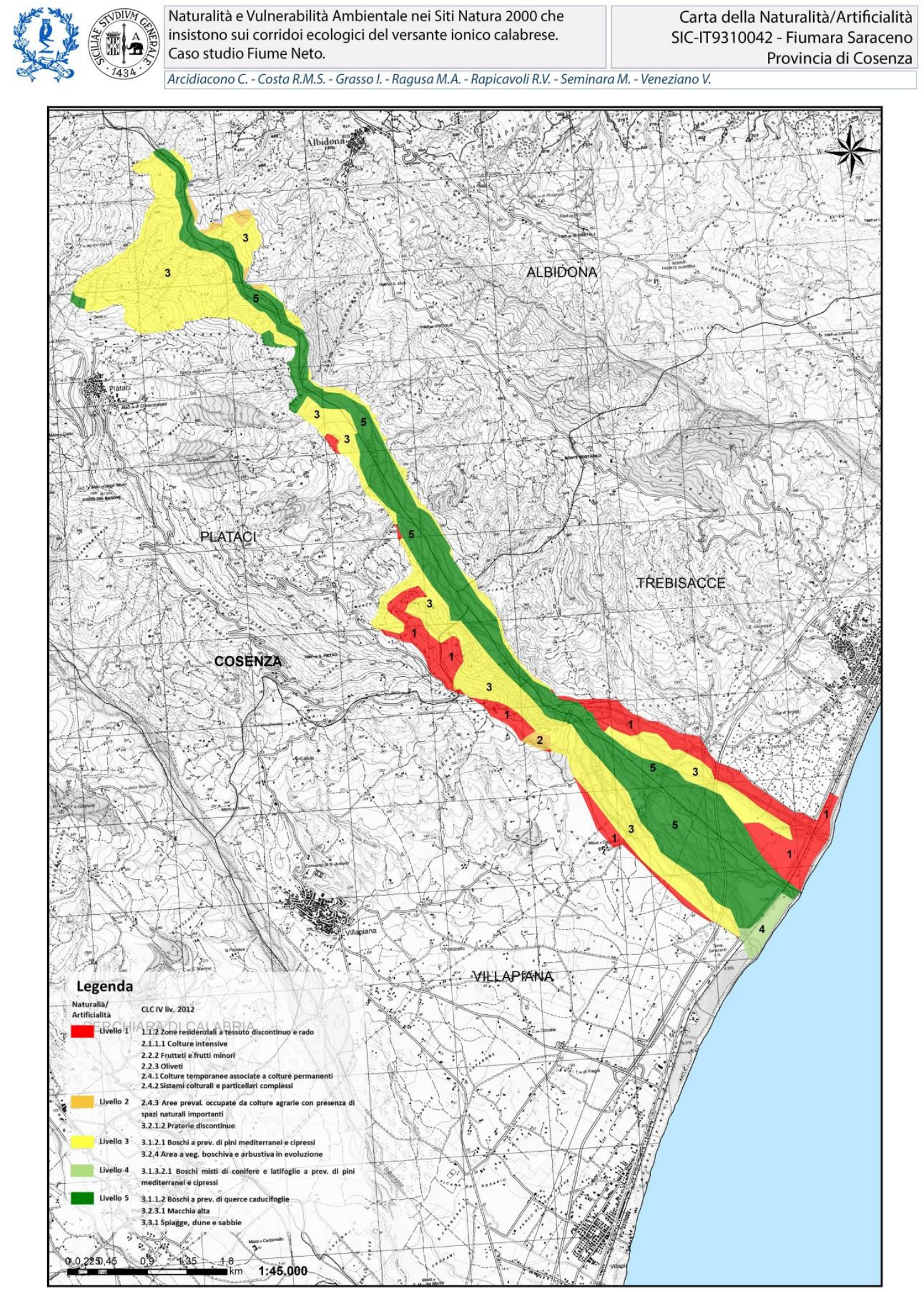

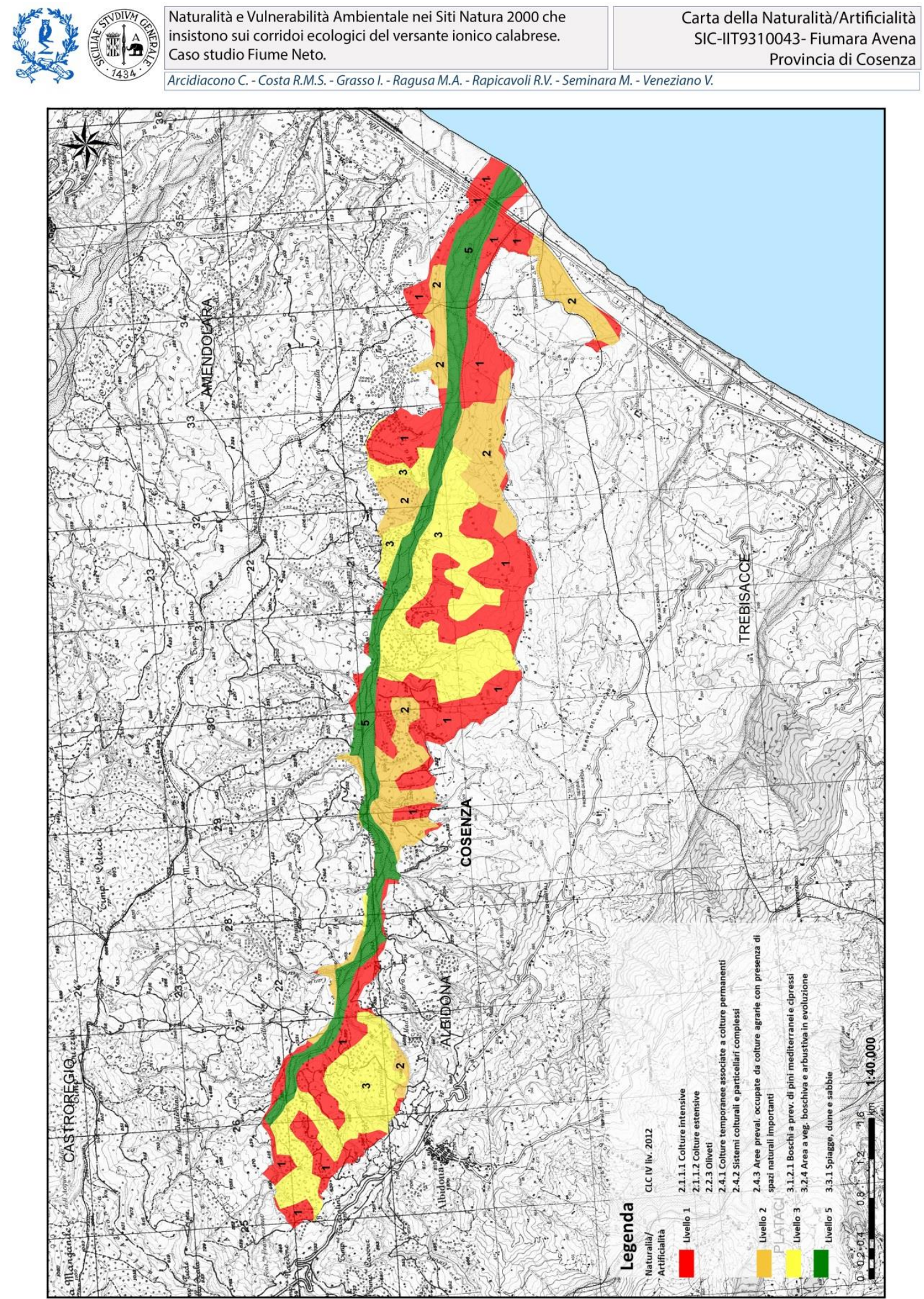

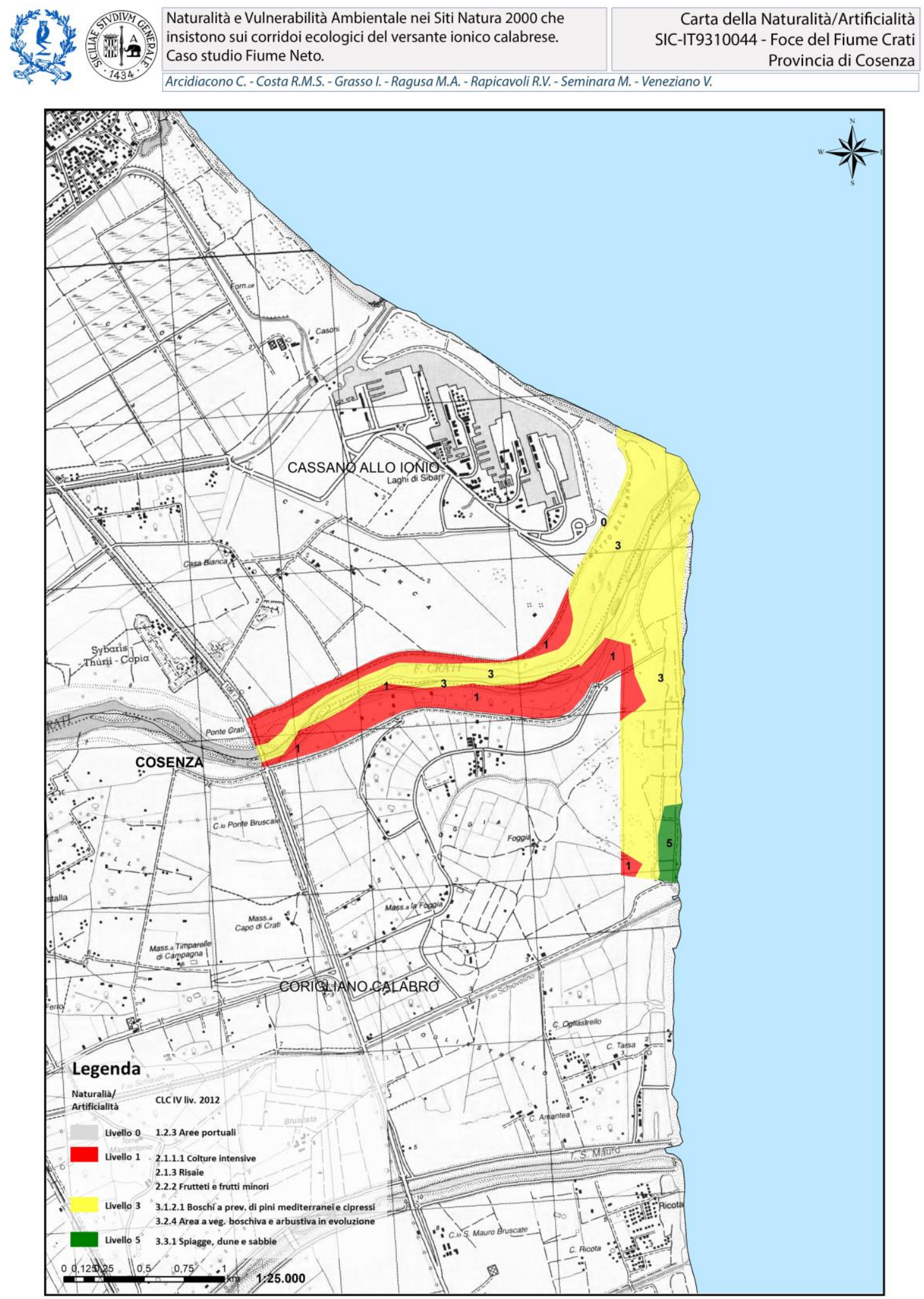

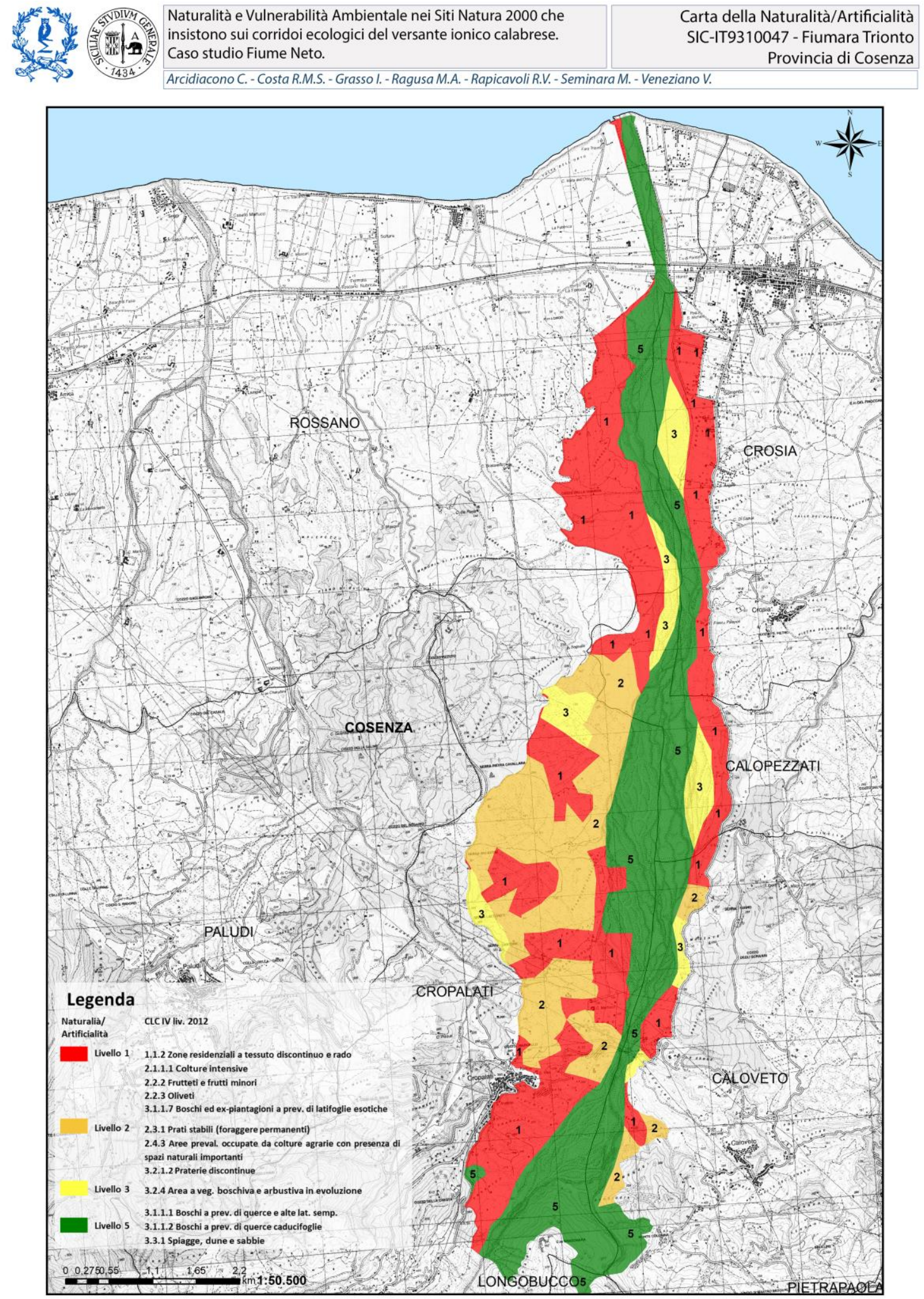

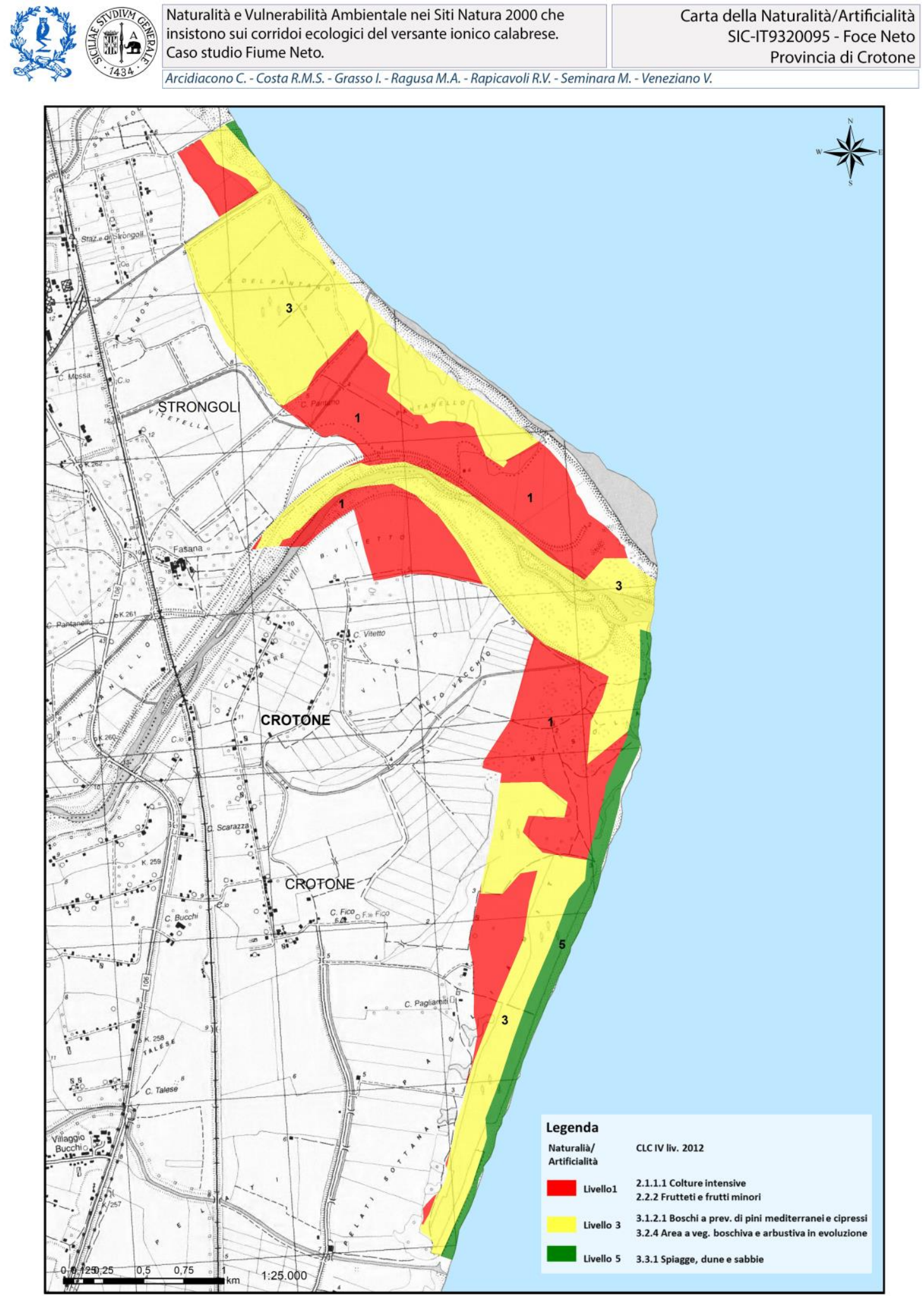


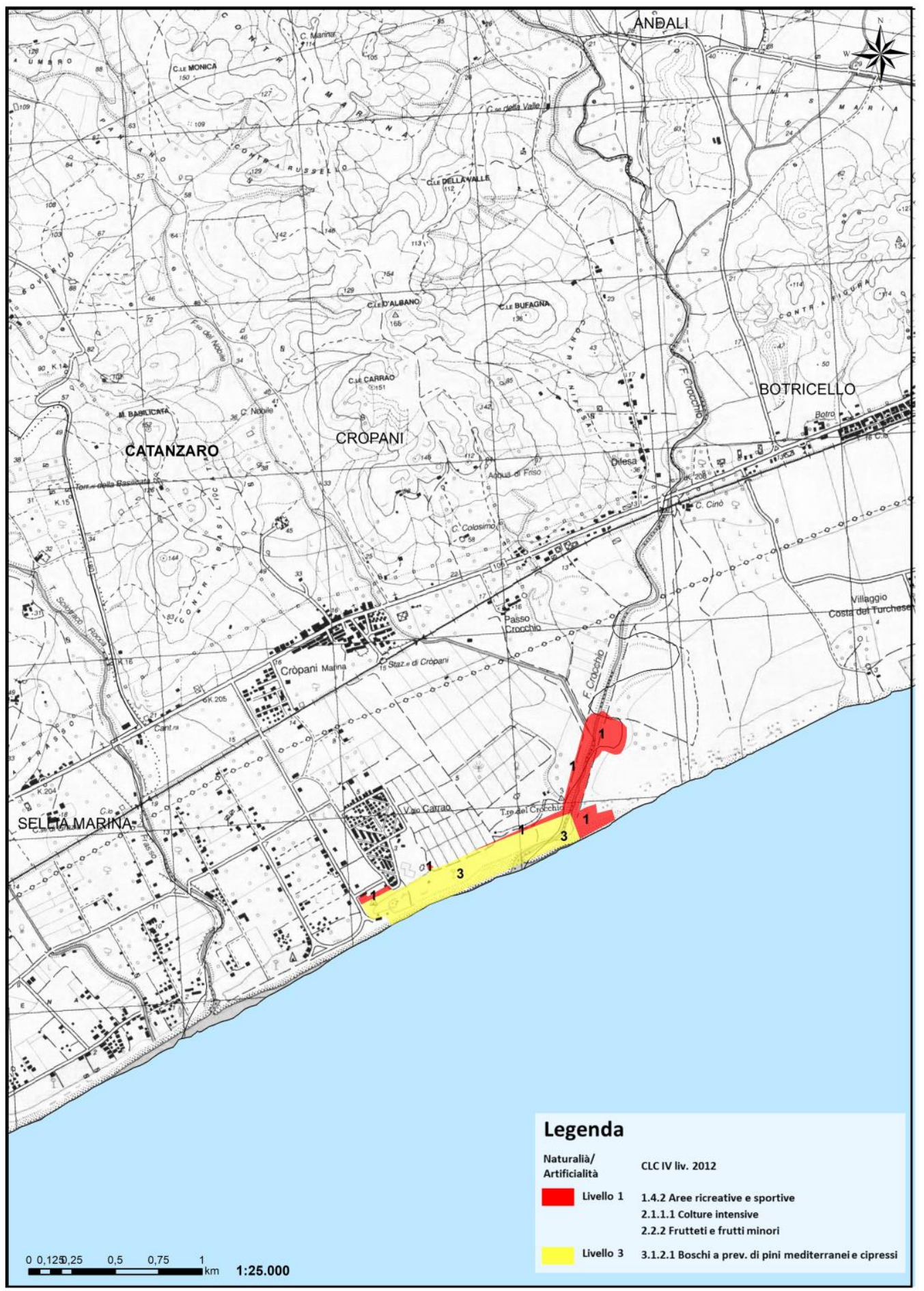



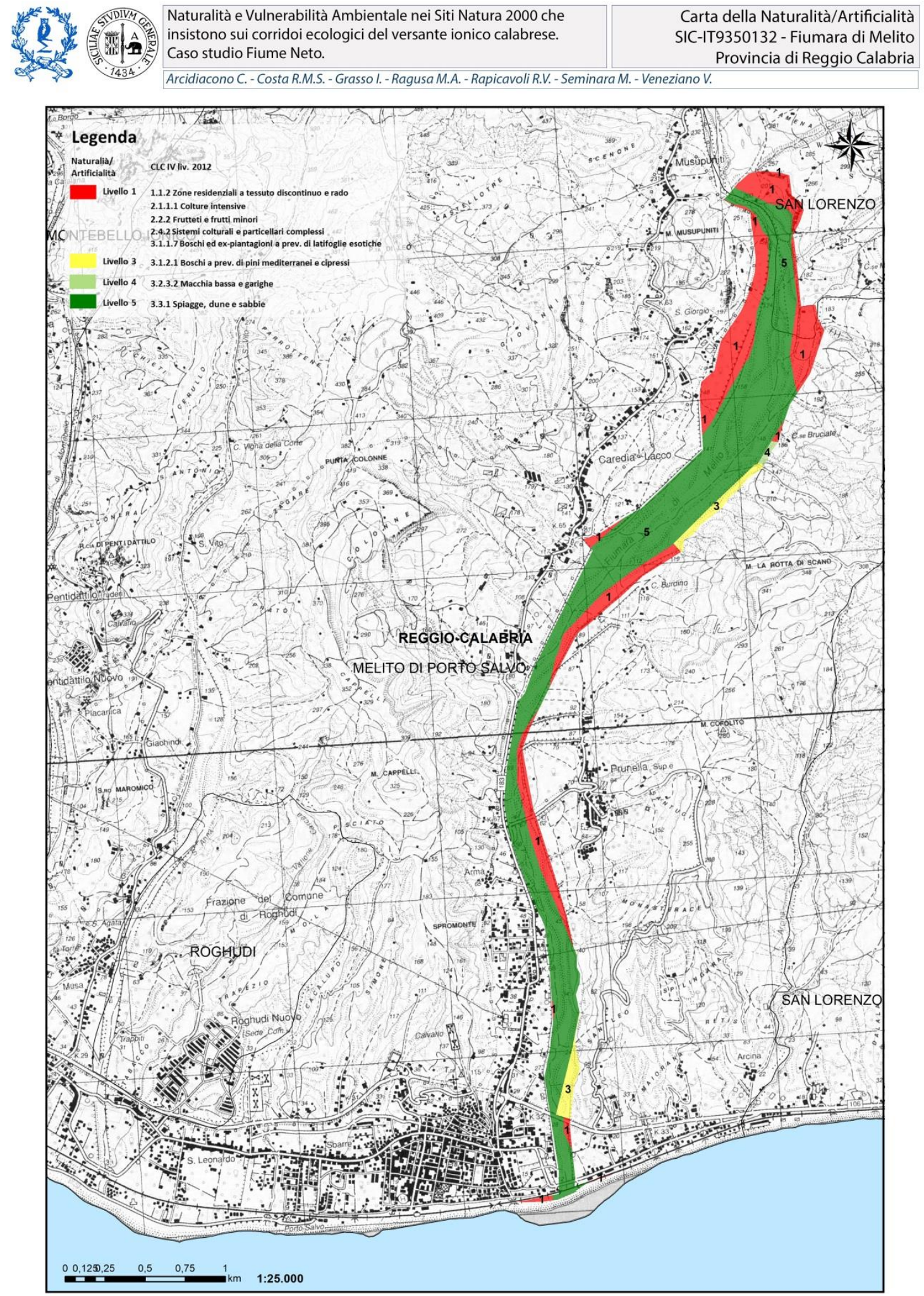


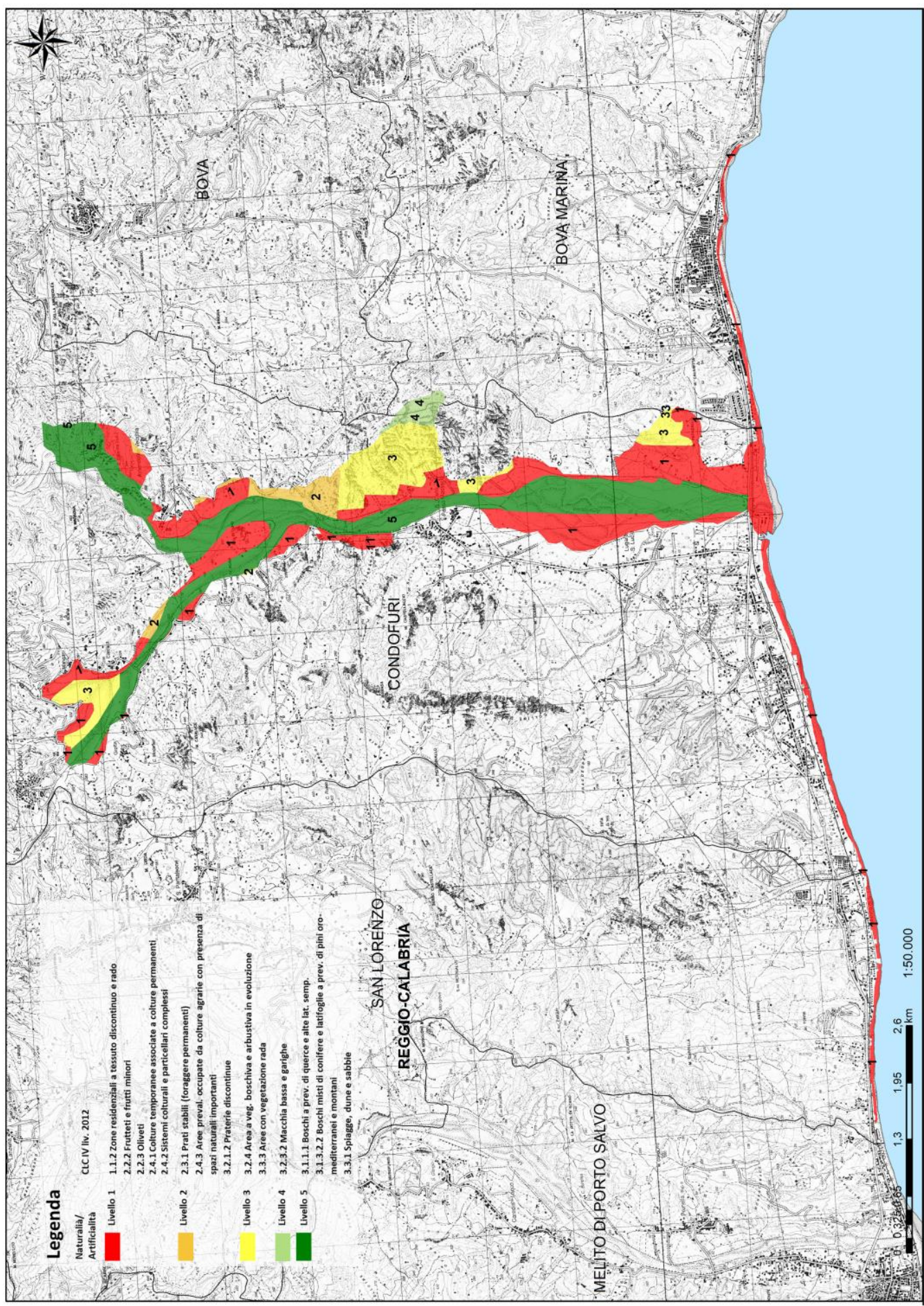




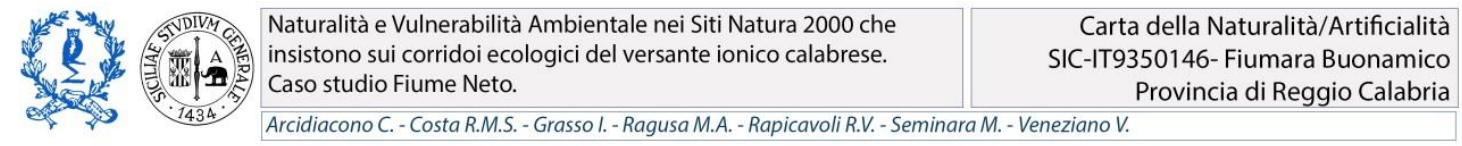

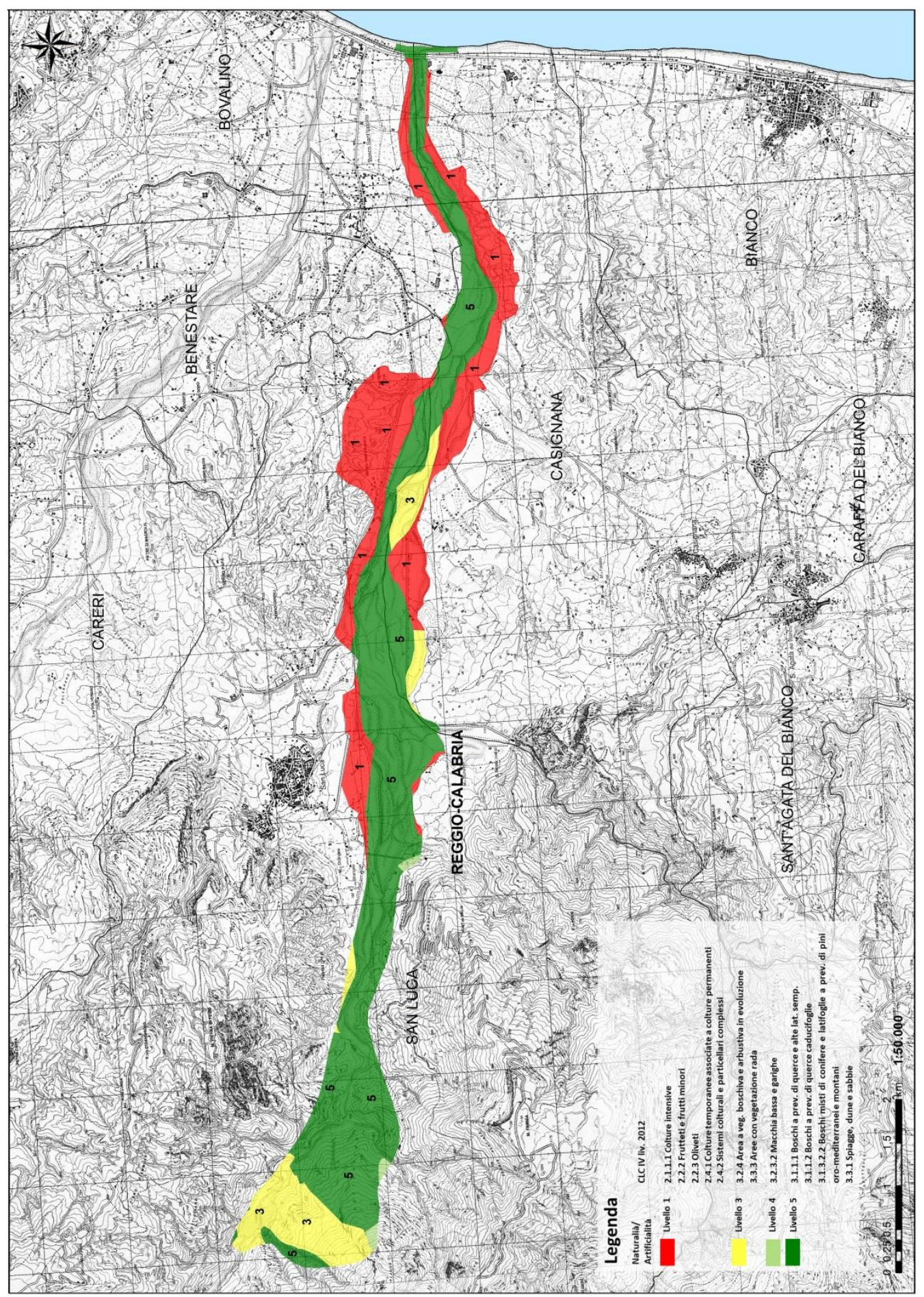




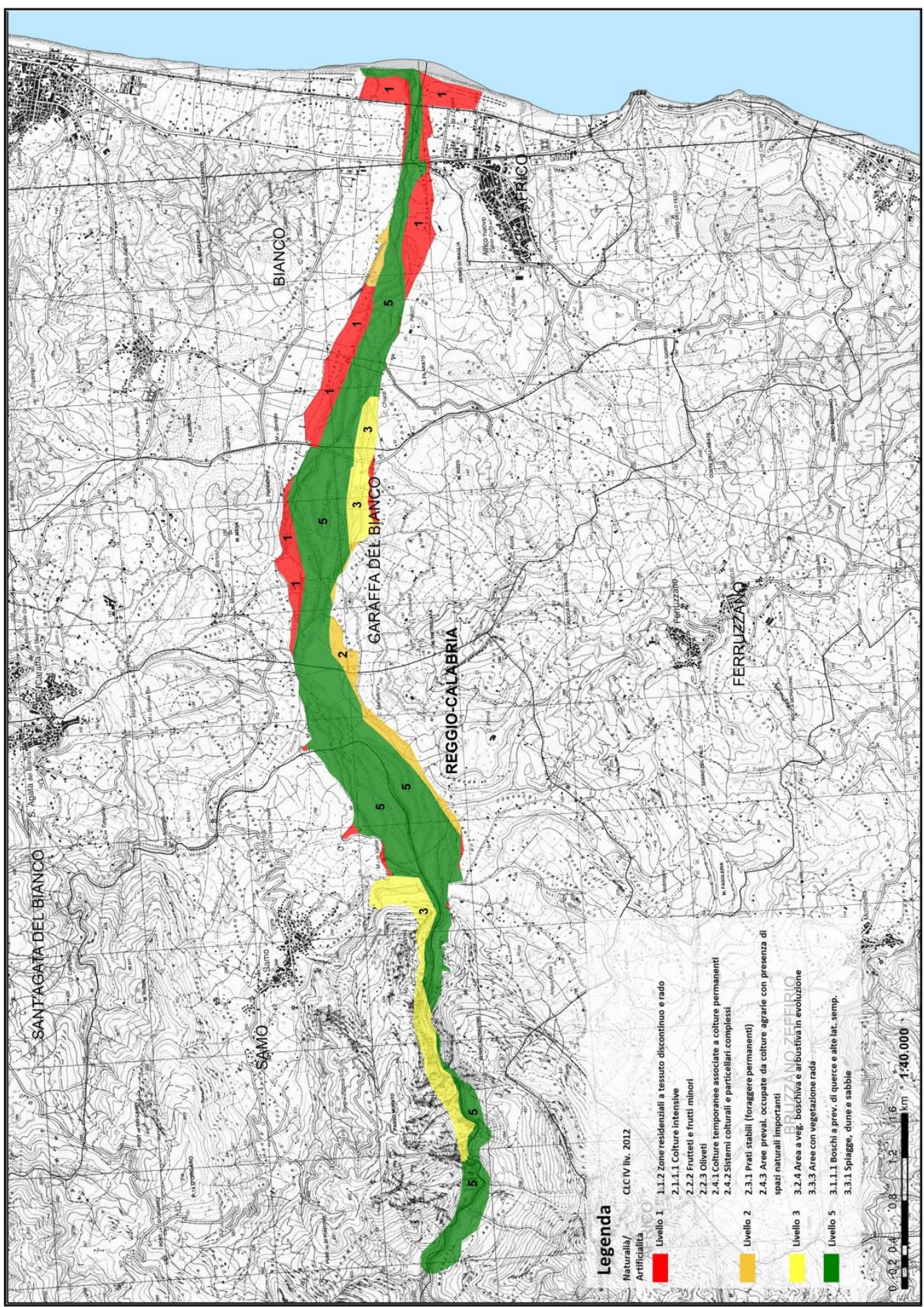


IT9310042 Fiumara Saraceno

IT9310043 Fiumara Avena

IT9310044 Fiume Crati

IT9310047 Fiumara Trionto

IT9320095 Foce Neto

IT9330105 Foce del Crocchio - Cropani

IT9350132 Fiumara di Melito

IT9350145 Fiumara Amendolea

IT9350146 Fiumara Buonamico

IT9350147 Fiumara Laverde 


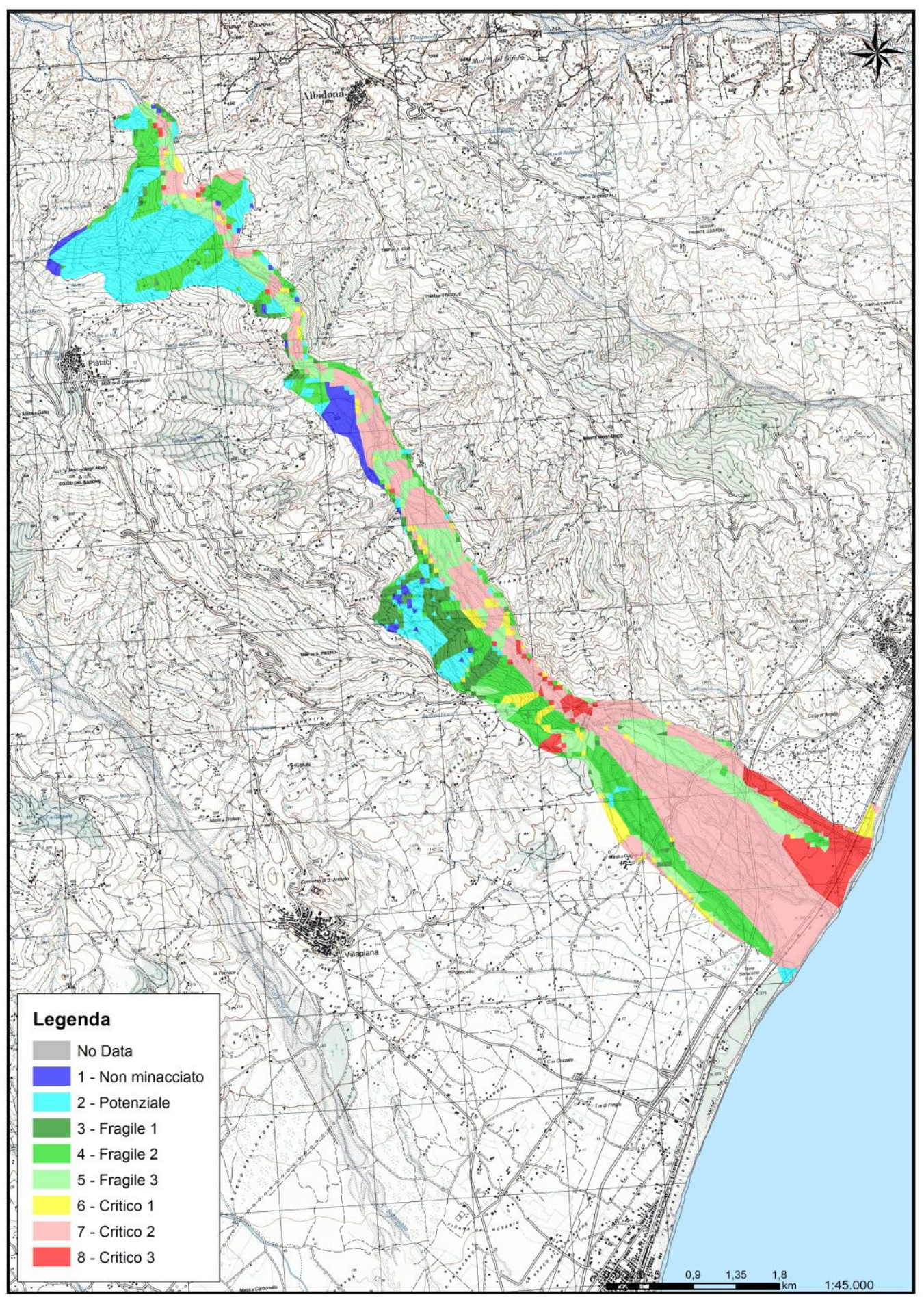



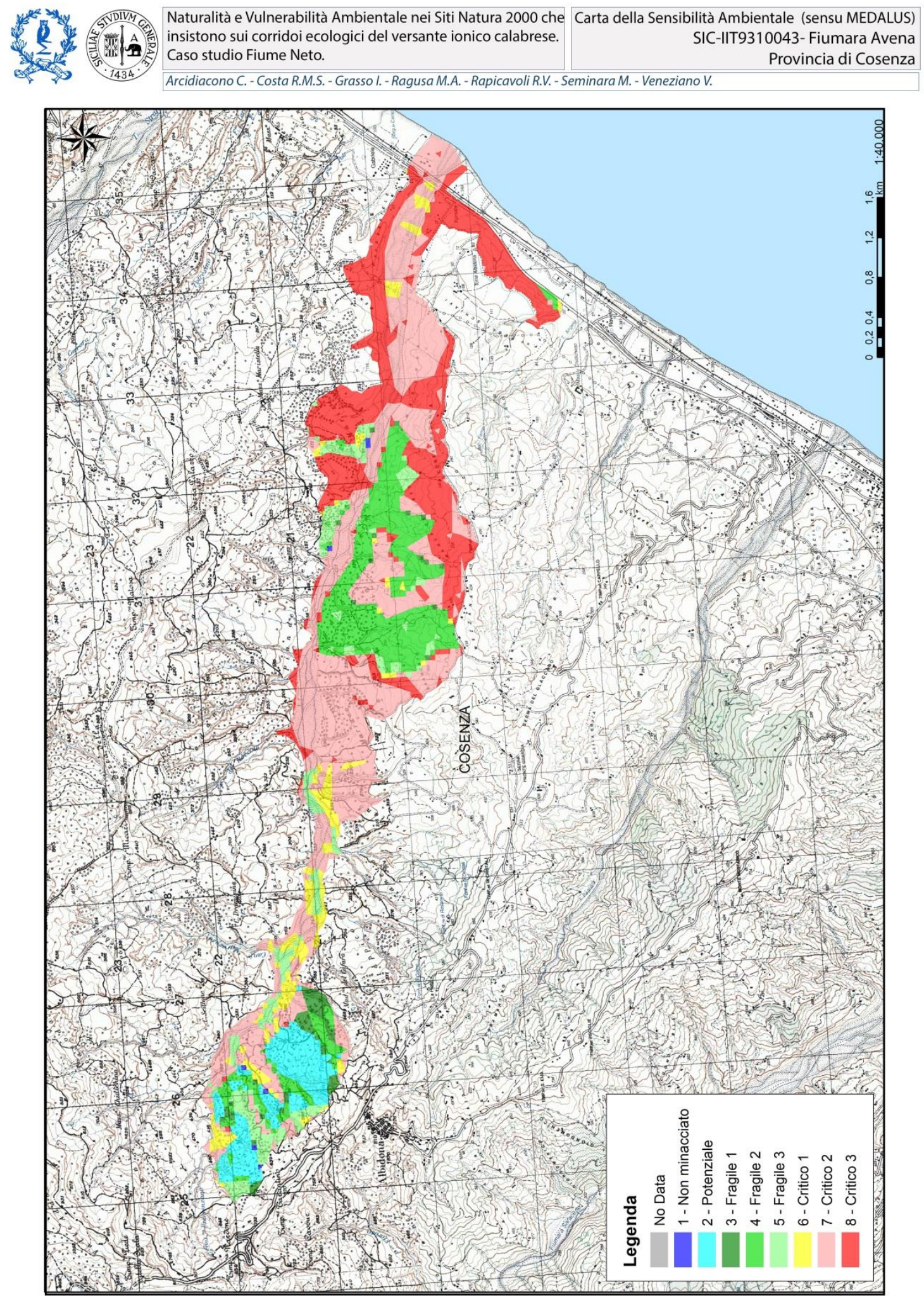

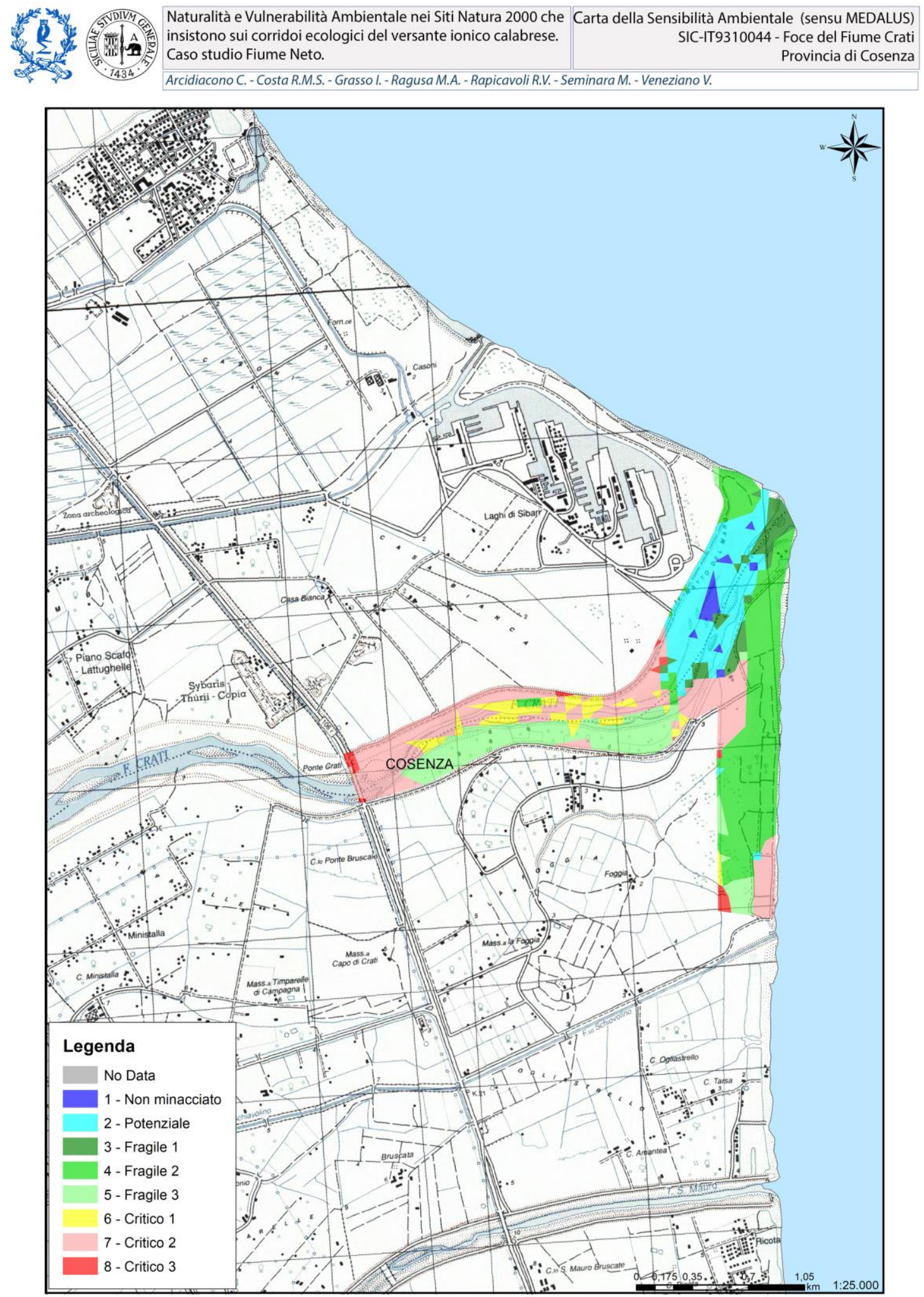

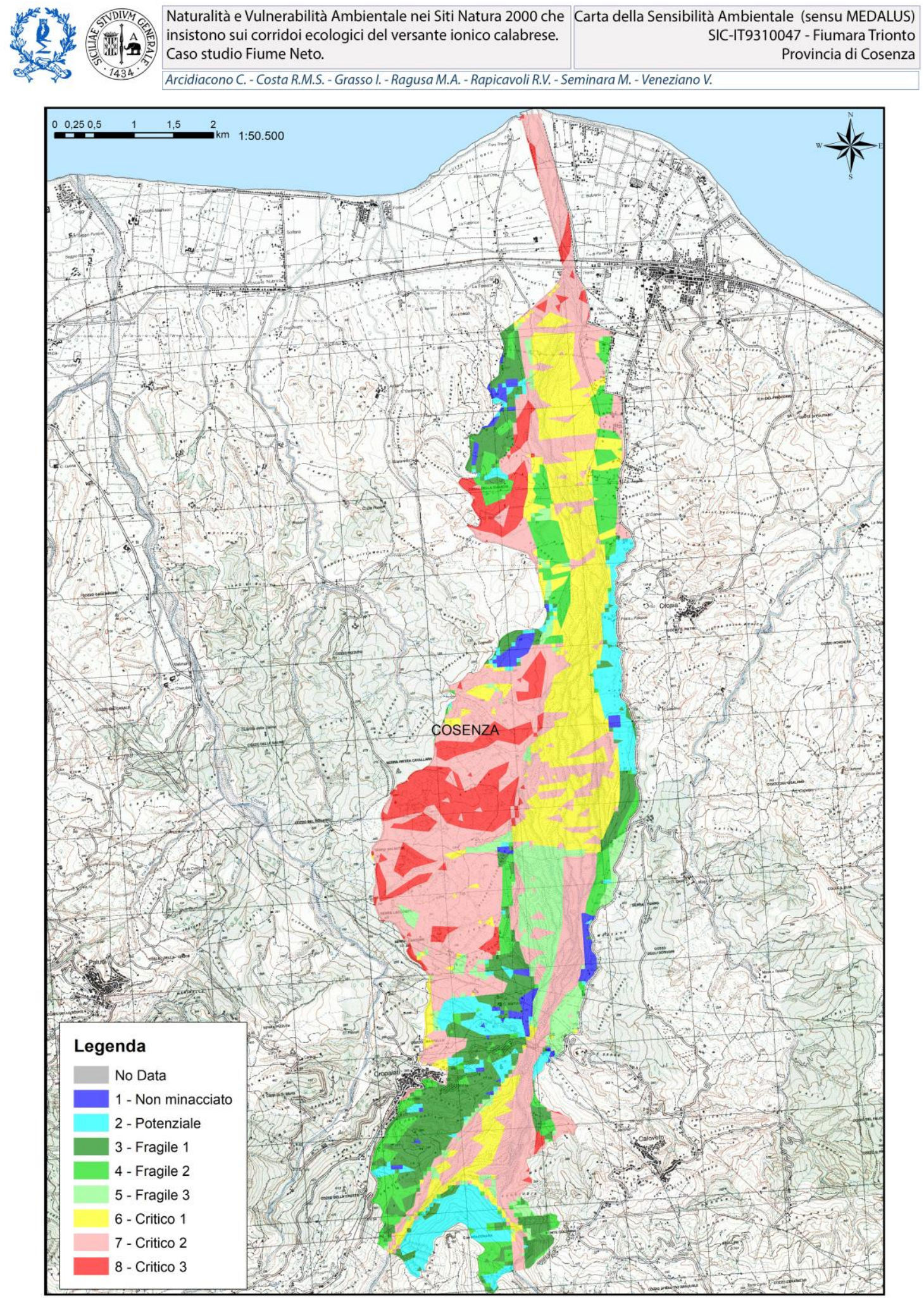

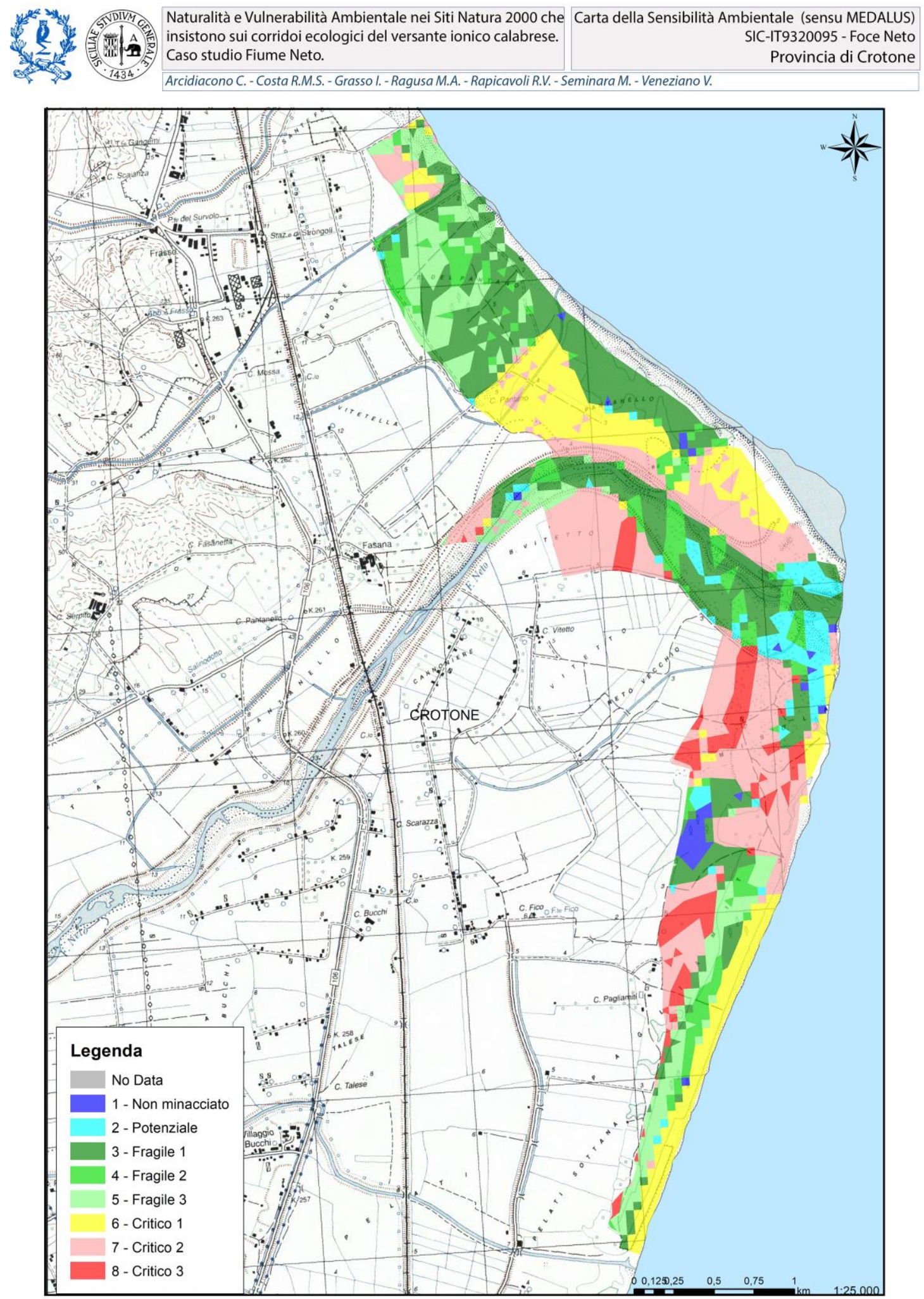

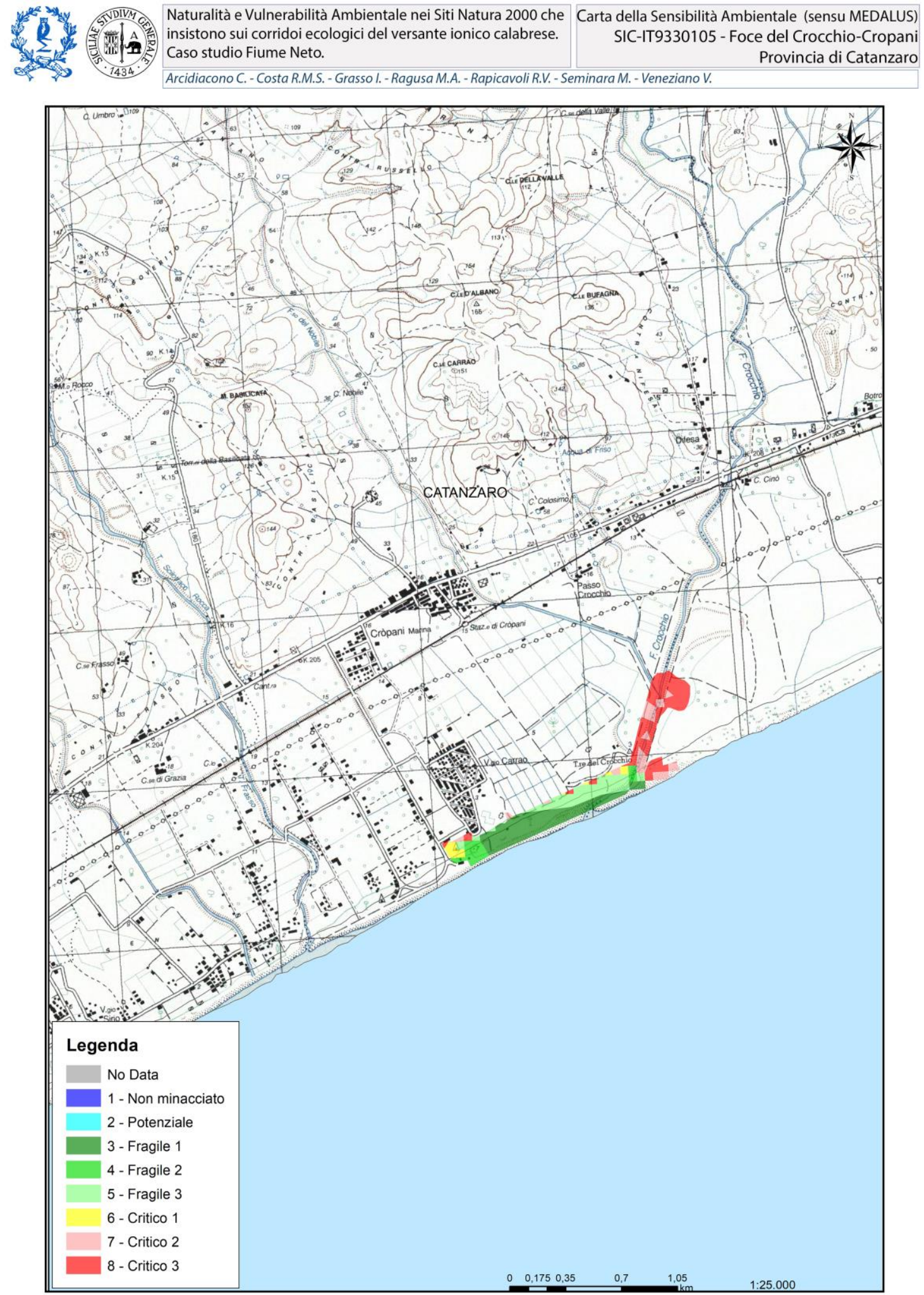

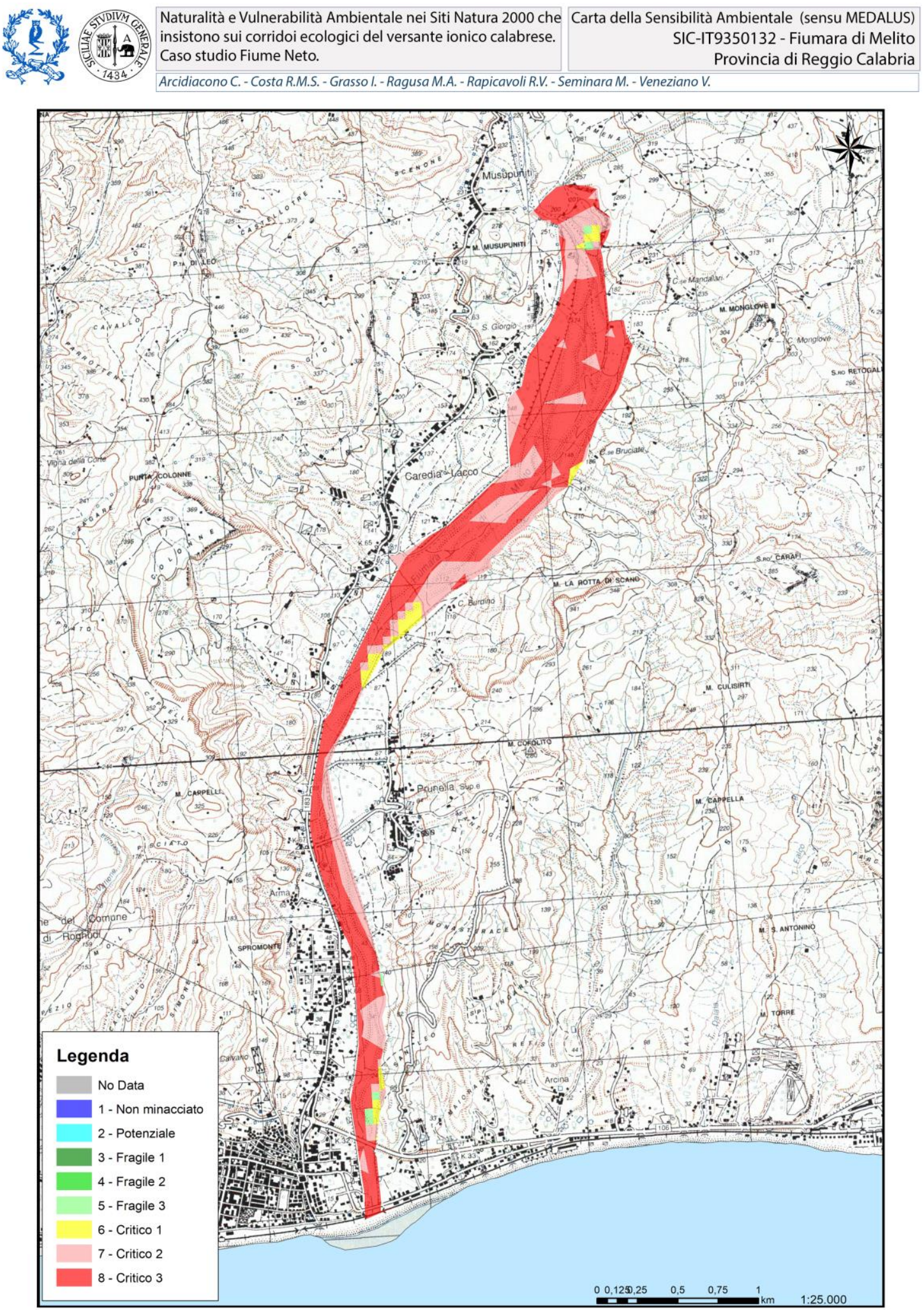

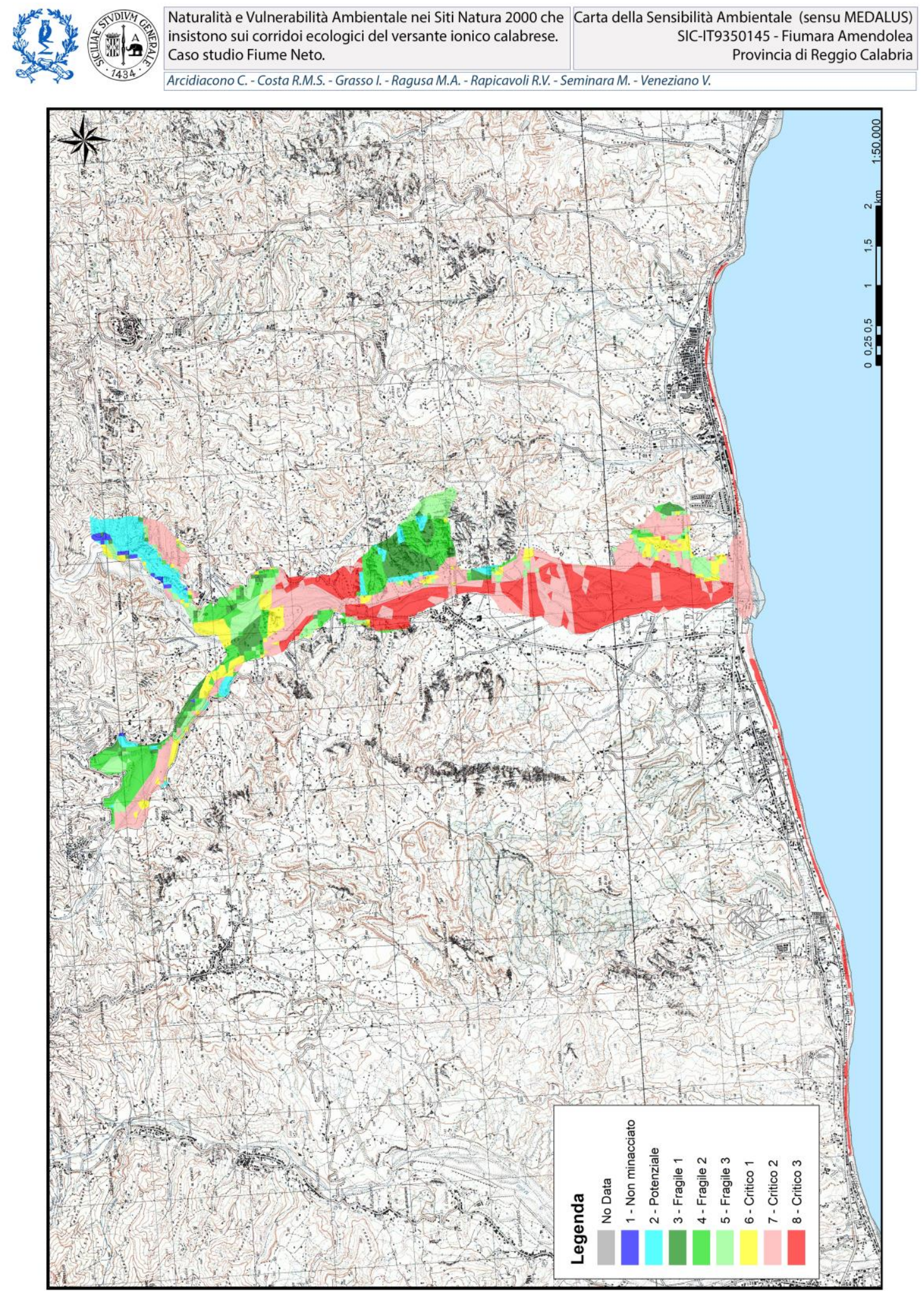

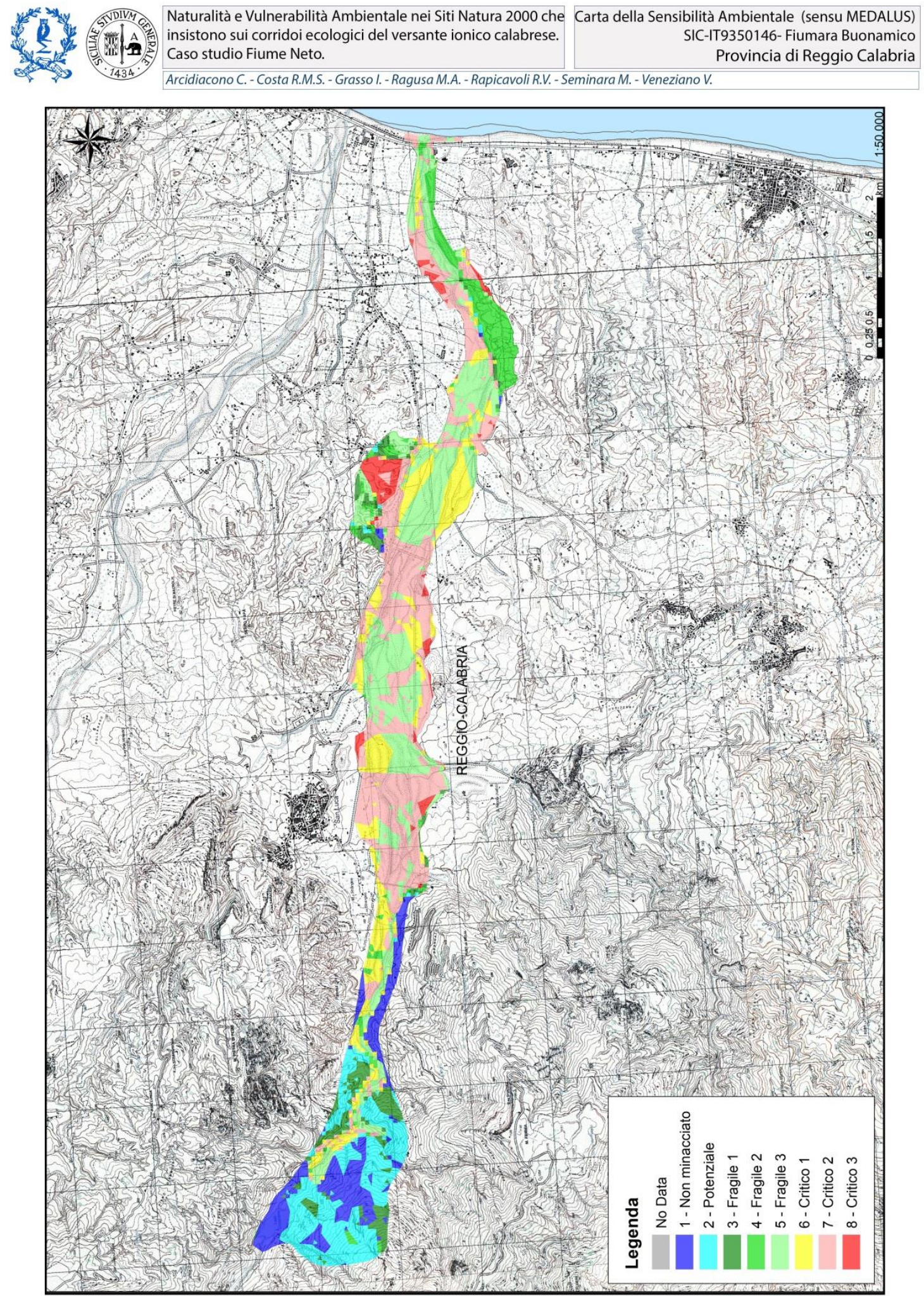

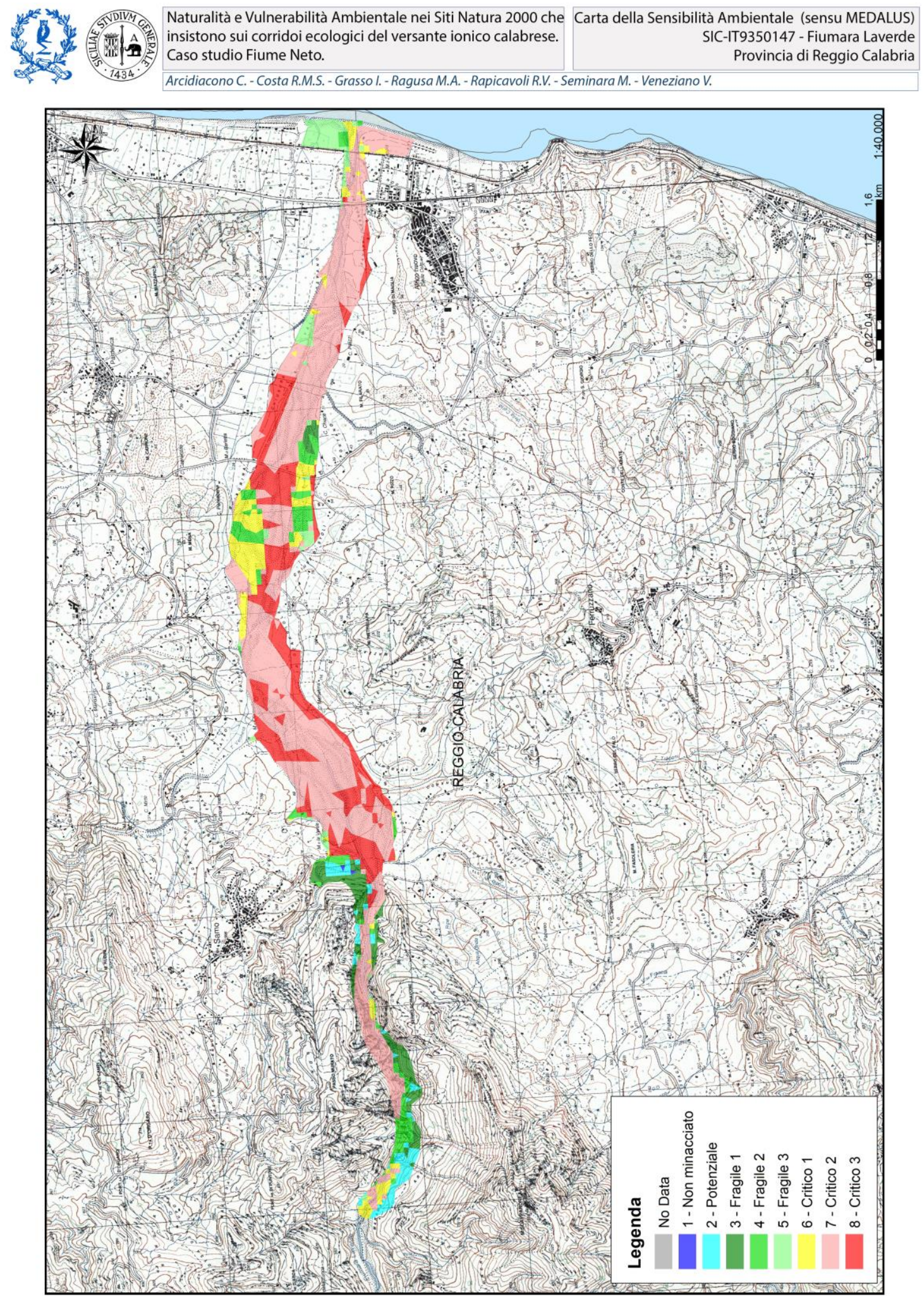
Tab. 1. Salsolo kali-Cakiletum maritimae Costa \& Mansanet 1981, corr. Rivas-Martínez et al. 1992

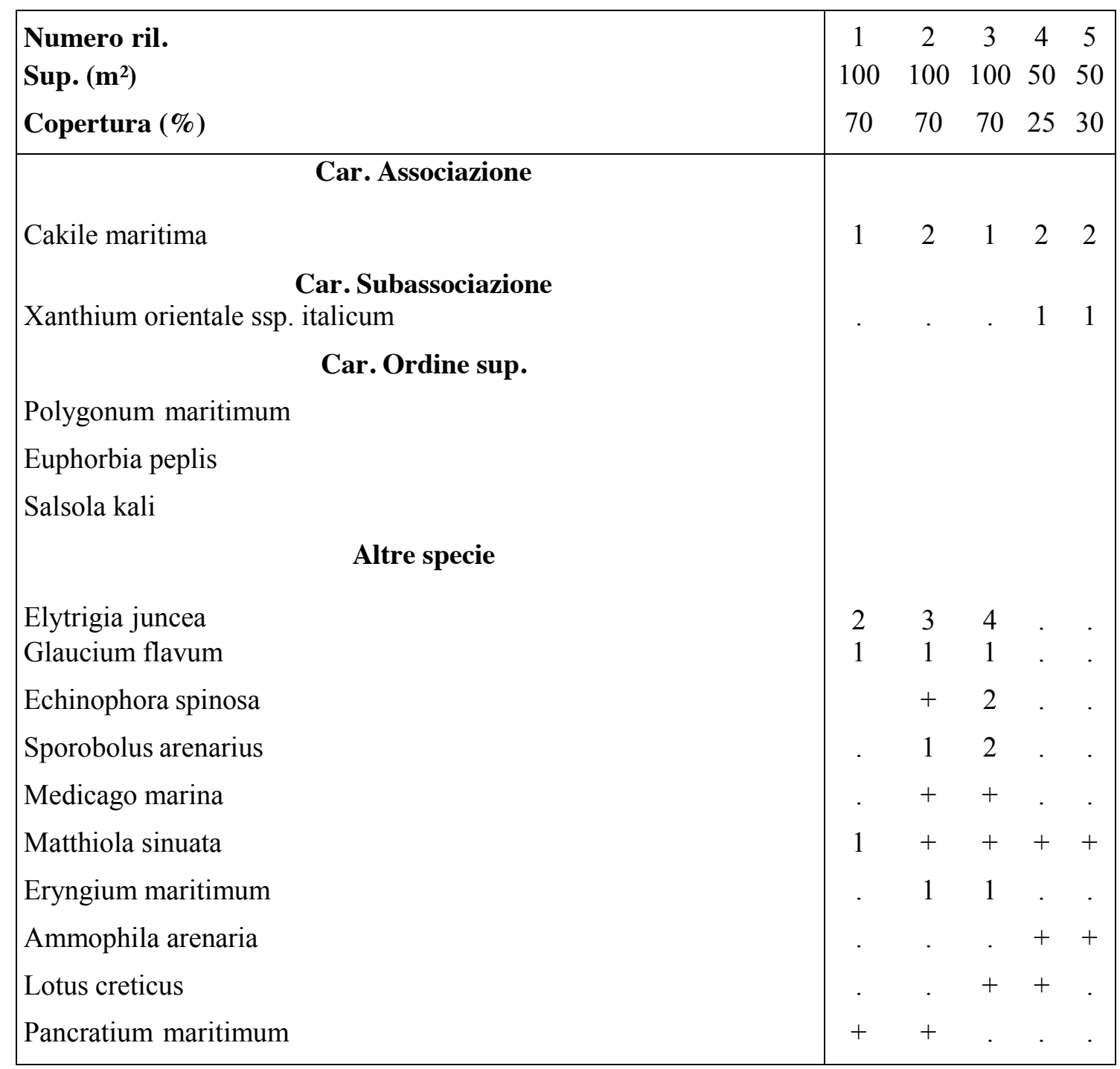


Tab.2. Echinophoro spinosae-Elytrigietum junceae Géhu 1988 corr. Géhu 1996 Echinophoro spinosae-Ammophiletum australis (Br.-B1. 1933) Géhu, Rivas-Martinez \& R. Tx. 1972 in Géhu et al. 1984

\begin{tabular}{|c|c|c|c|c|c|c|c|c|c|c|c|}
\hline $\begin{array}{l}\text { Numero ril. } \\
\text { Sup. }\left(\mathbf{m}^{2}\right)\end{array}$ & $\begin{array}{c}6 \\
50\end{array}$ & $\begin{array}{c}7 \\
50\end{array}$ & $\begin{array}{c}8 \\
50\end{array}$ & $\begin{array}{c}9 \\
100\end{array}$ & $\begin{array}{c}10 \\
100\end{array}$ & $\begin{array}{c}11 \\
100\end{array}$ & $\begin{array}{l}12 \\
50\end{array}$ & $\begin{array}{l}13 \\
50\end{array}$ & $\begin{array}{l}14 \\
50\end{array}$ & $\begin{array}{l}15 \\
20\end{array}$ & $\begin{array}{l}16 \\
30\end{array}$ \\
\hline Copertura (\%) & 70 & 70 & 70 & 60 & 60 & 60 & 70 & 70 & 70 & 80 & 70 \\
\hline \multicolumn{12}{|l|}{ Car. Associazione } \\
\hline Elytrigia juncea & 3 & 2 & 4 & 2 & 2 & 3 & 3 & 2 & 2 & 3 & 4 \\
\hline Echinophora spinosa & 1 & 1 & 2 & 1 & + & + & 2 & 1 & 1 & 3 & 1 \\
\hline \multicolumn{11}{|l|}{ Car. Ordine sup. } & 3 \\
\hline Otanthus maritimus & 3 & 3 & 2 & 3 & 3 & 2 & 2 & 1 & 3 & 2 & 1 \\
\hline Matthiola sinuata & 1 & 1 & 1 & . & . & 1 & 1 & + & + & + & . \\
\hline Cyperus kalli & 1 & + & + & . & . & . & + & + & + & . & . \\
\hline Pancratium maritimum & + & + & 1 & 1 & + & + & . & . & . & . & . \\
\hline Eryngium maritimum & + & + & 1 & 1 & + & + & + & 1 & . & . & . \\
\hline Polygonum maritimum & + & . & . & . & . & . & . & . & . & . & . \\
\hline \multicolumn{12}{|l|}{ Altre specie } \\
\hline Vulpia membranacea & 1 & + & + & + & + & . & . & 1 & + & + & . \\
\hline Glaucium flavum & + & + & . & . & . & . & . & . & . & + & . \\
\hline Reseda alba & + & + & 1 & 1 & . & + & + & . & . & . & . \\
\hline Artemisia campestris subsp. variabilis & & 1 & 1 & + & + & . & . & . & . & . & . \\
\hline Medicago marina & & + & + & . & . & . & . & . & . & 1 & . \\
\hline Matthiola sinuata & & . & + & + & + & . & . & . & . & 1 & + \\
\hline Pancratium maritimum & + & + & & . & . & . & . & . & . & . & . \\
\hline
\end{tabular}


Tab. 3. Sileno nicaeensis-Ononidetum variegatae Géhu et al.1986 Sileno coloratae-Vulpietum membranaceae (Pign. 1953) Géhu \& Scoppola 1984

\begin{tabular}{|c|c|c|c|c|c|c|}
\hline $\begin{array}{l}\text { Numero ril. } \\
\text { Sup. }\left(\mathbf{m}^{2}\right) \\
\text { Copertura }(\%)\end{array}$ & $\begin{array}{l}17 \\
30 \\
70\end{array}$ & $\begin{array}{l}18 \\
40 \\
60\end{array}$ & $\begin{array}{l}19 \\
40 \\
70\end{array}$ & $\begin{array}{l}20 \\
10 \\
40\end{array}$ & $\begin{array}{l}21 \\
10 \\
70\end{array}$ & $\begin{array}{l}22 \\
20 \\
70\end{array}$ \\
\hline \multicolumn{7}{|l|}{ Car. Associazione } \\
\hline Ononis variegata & 1 & 2 & 2 & . & . & . \\
\hline Silene colorata & + & 1 & + & 1 & 1 & 1 \\
\hline Silene nicaeensis & 1 & 1 & + & 1 & 1 & + \\
\hline Vulpia fasciculata &.+ & 1 & 1 & 2 & 2 & 1 \\
\hline \multicolumn{7}{|l|}{ Car. Ordine sup. } \\
\hline Matthiola sinuata & 1 & 1 & 1 & . & . & . \\
\hline Corynephorus articulatus & 1 & + & + & 2 & 1 & 1 \\
\hline Lagurus ovatus & + & + & 1 & 1 & + & + \\
\hline Rumex bucephalophorus & + & + & 1 & 2 & 1 & 1 \\
\hline Corynephorus divaricatus & 1 & + & + & 2 & 1 & 1 \\
\hline Chondrilla juncea & + & . & + & . & . & . \\
\hline \multicolumn{7}{|l|}{ Altre specie } \\
\hline Reseda alba & + & + & + & . & . & . \\
\hline Artemisia campestris subsp. variabilis & + & 1 & 1 & . & . & . \\
\hline Medicago marina & . & + & + & . & . & . \\
\hline Matthiola sinuata & . & . & + & . & . & . \\
\hline Pancratium maritimum & + & . & . & + & + & . \\
\hline Oryzopsis miliacea & . & . & + & . & . & . \\
\hline
\end{tabular}


Tab. 4. Artemisio variabilis-Ephedretum distachyae Brullo et al. 2002

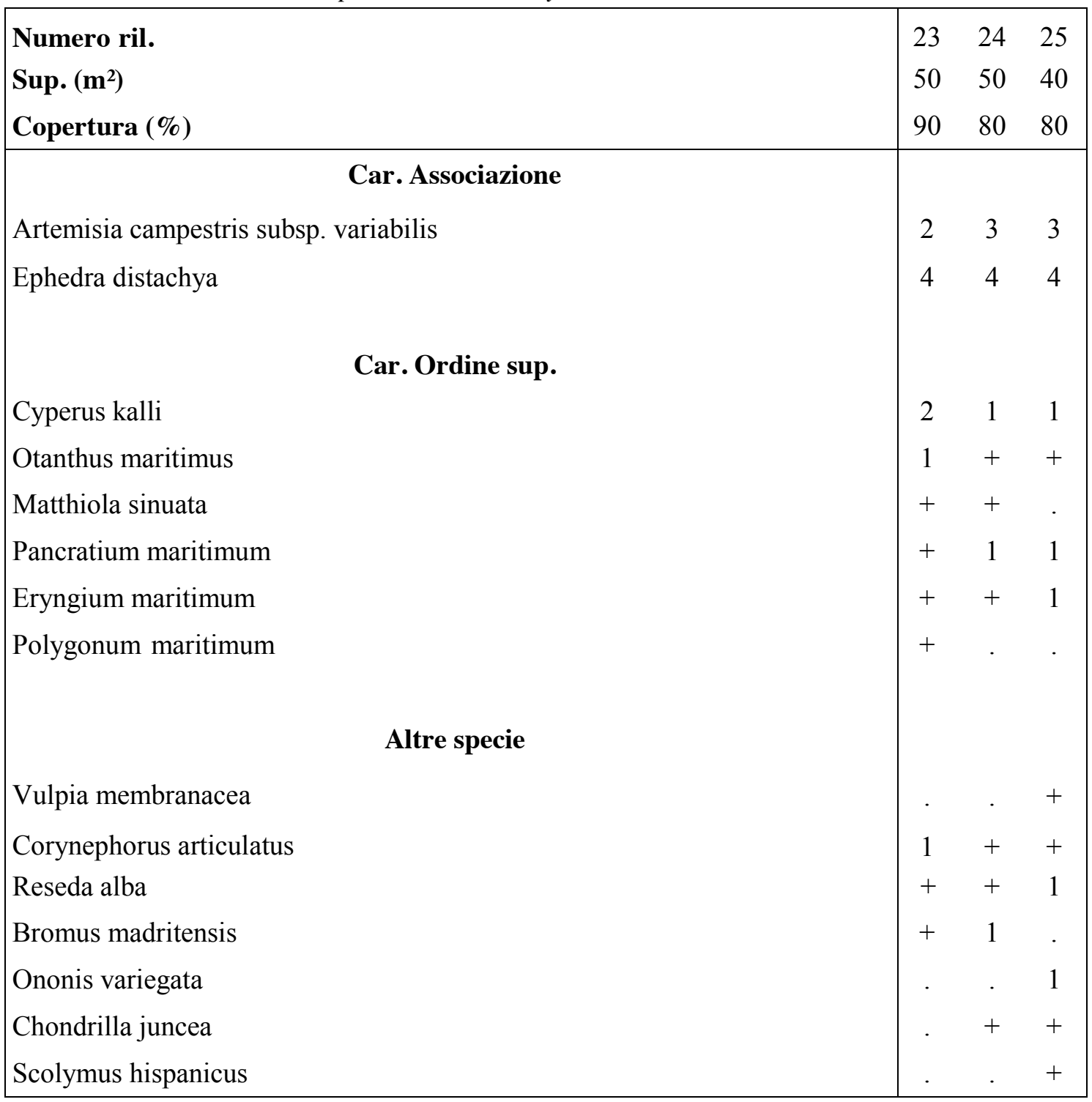


Tab. 5. Scirpetum compacti Van Langendonck 1931 corr. Bueno \& F. Prieto in Bueno 1997

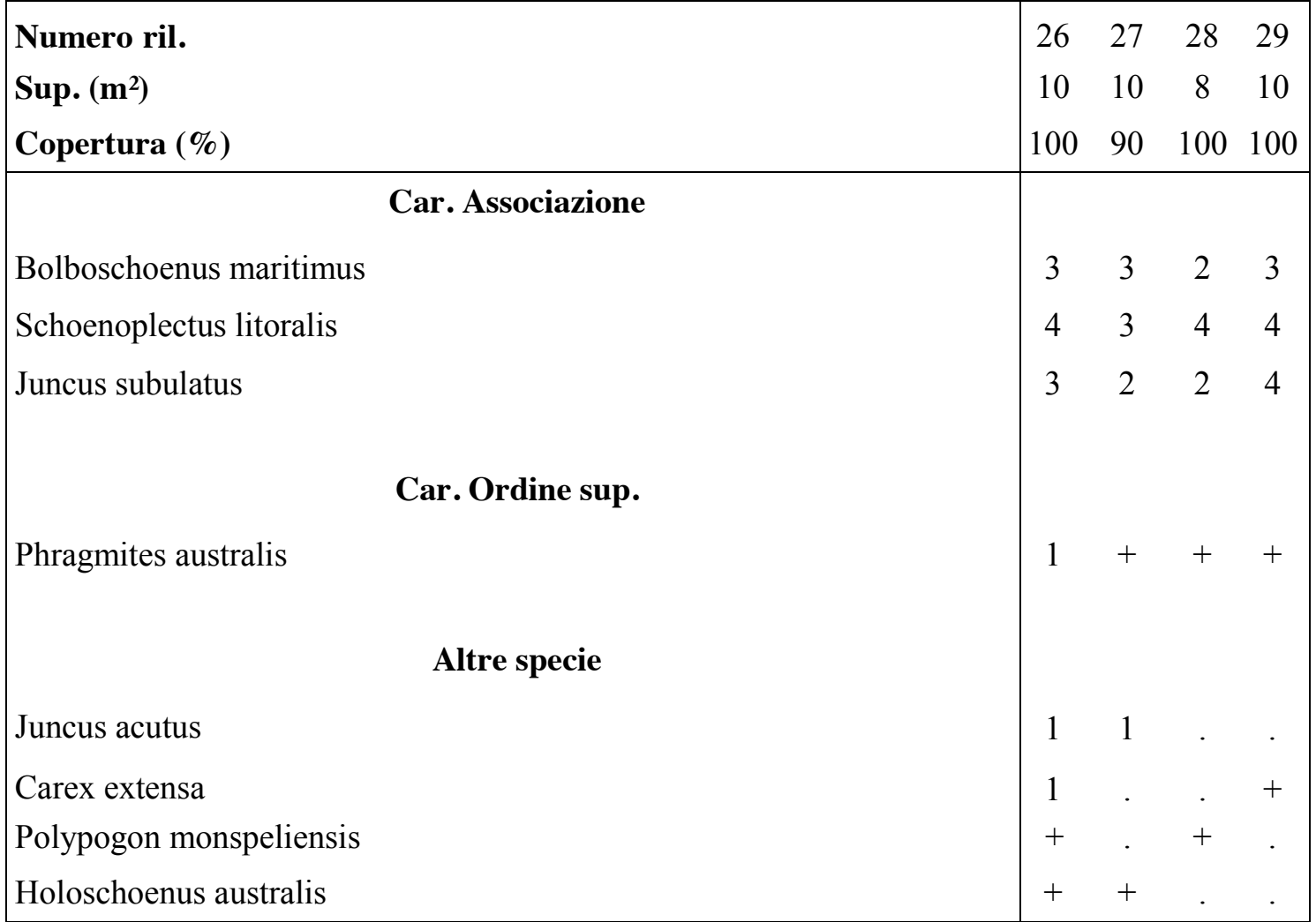

Tab. 6. Juncetum acuti Molinier \& Tallon 1970

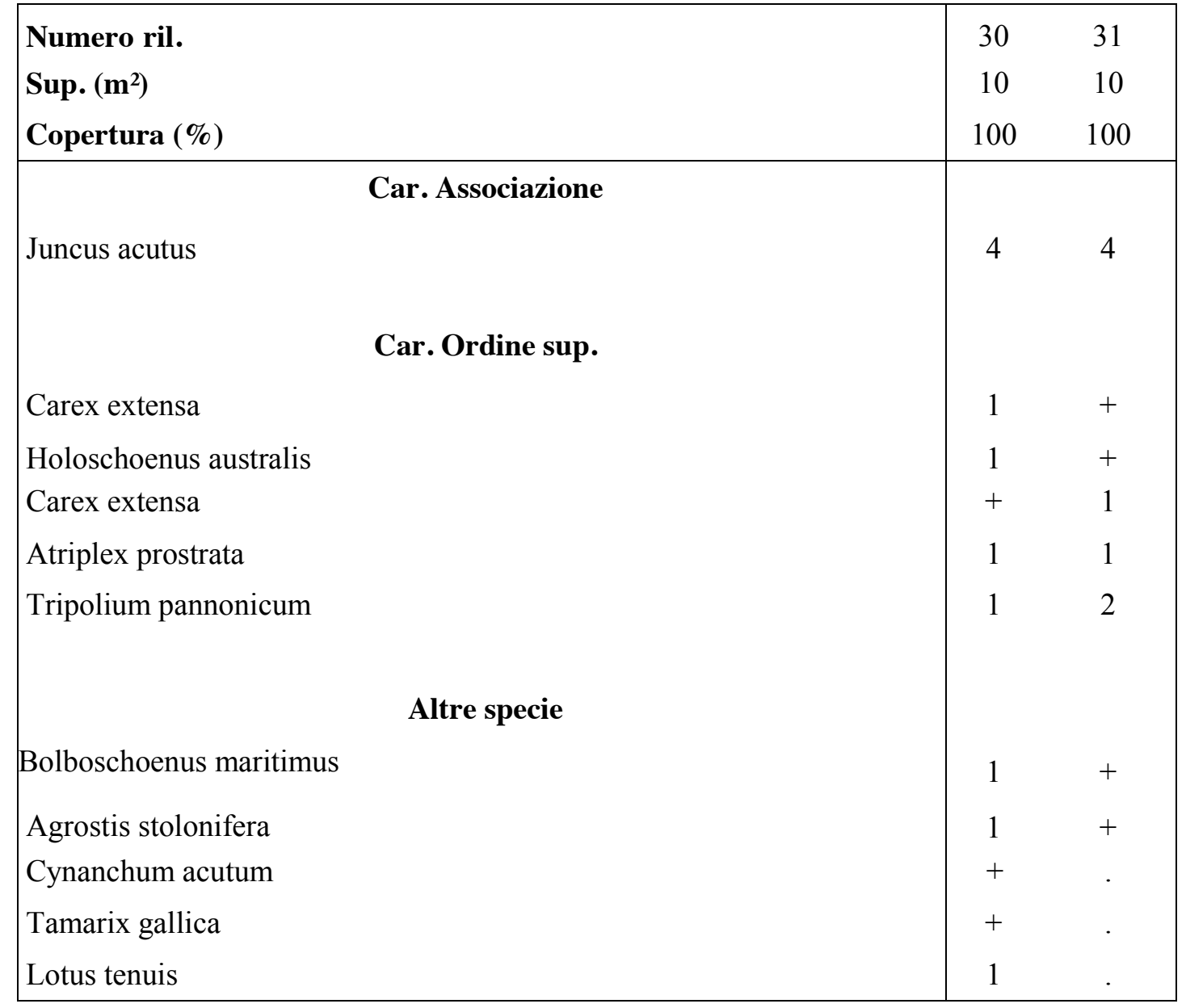


Tab. 7. Suaedo maritimae-Salicornietum patulae Brullo \& Furnari 1976 ex Géhu \& GéhuFranck 1984

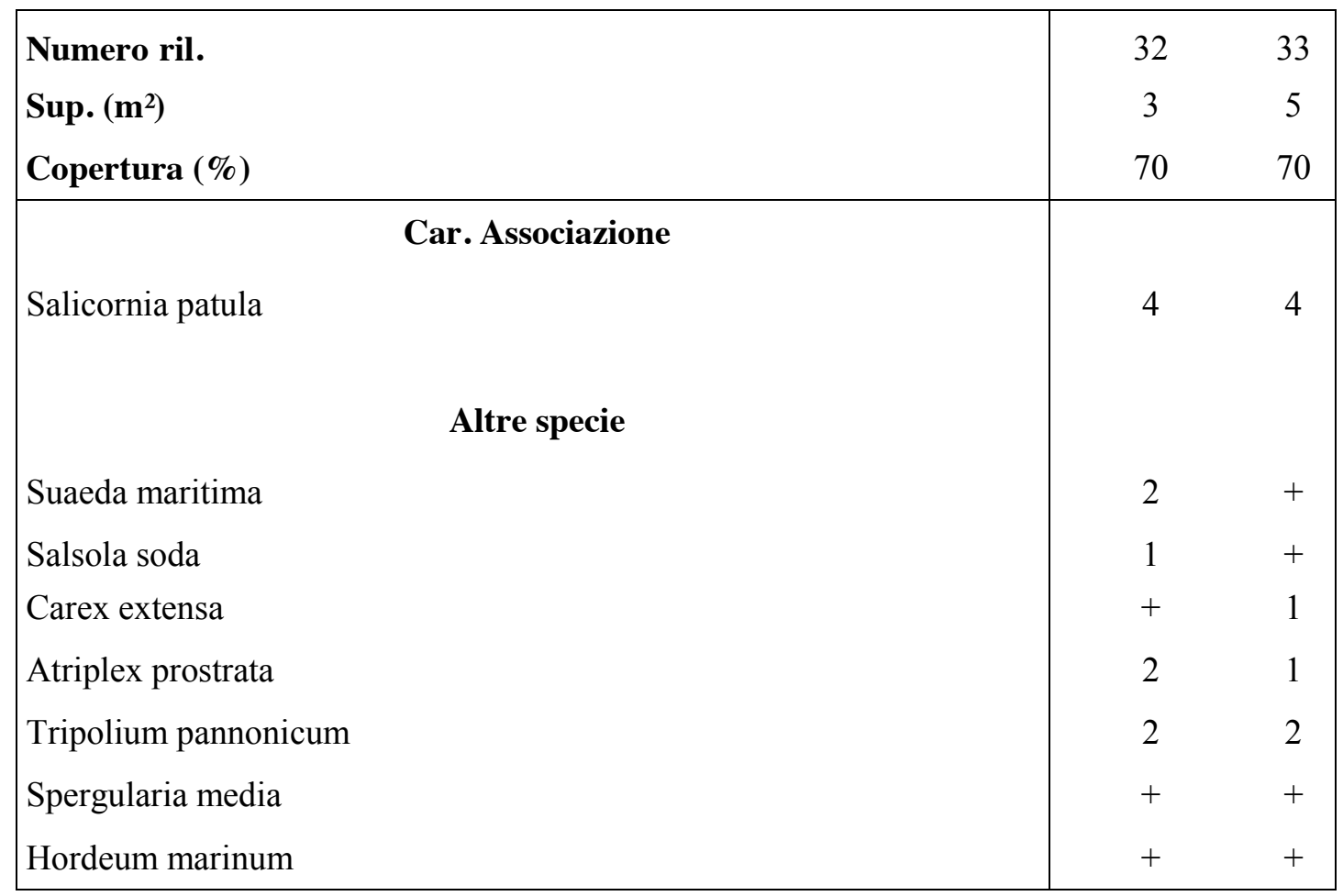

Tab. 8. Spergularietum salinae Molinier \& Tallon 1969

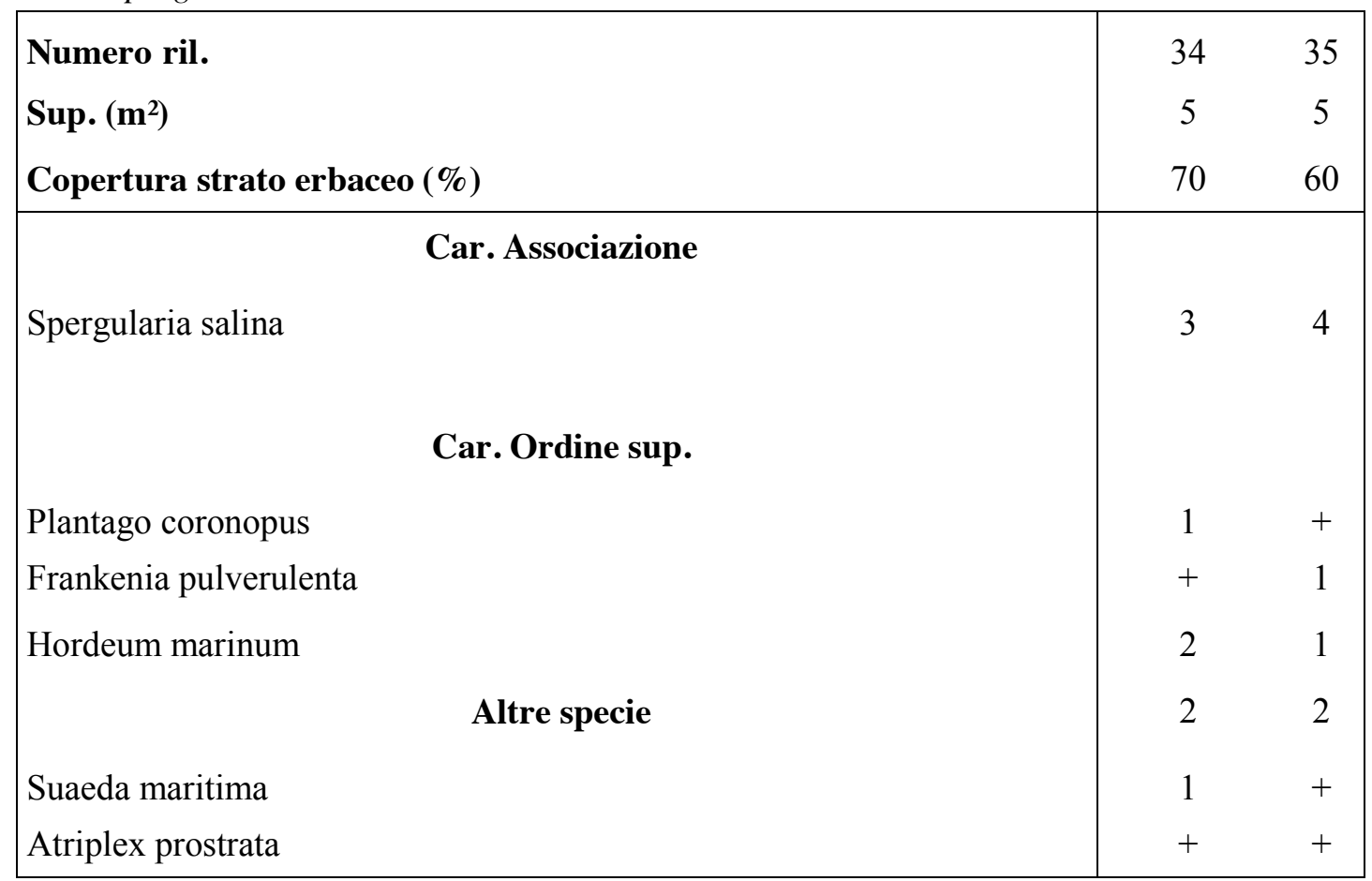


Tab. 9. Polygono salicifolii-Phragmitetum communis Barbagallo, Brullo \& Furnari 1979

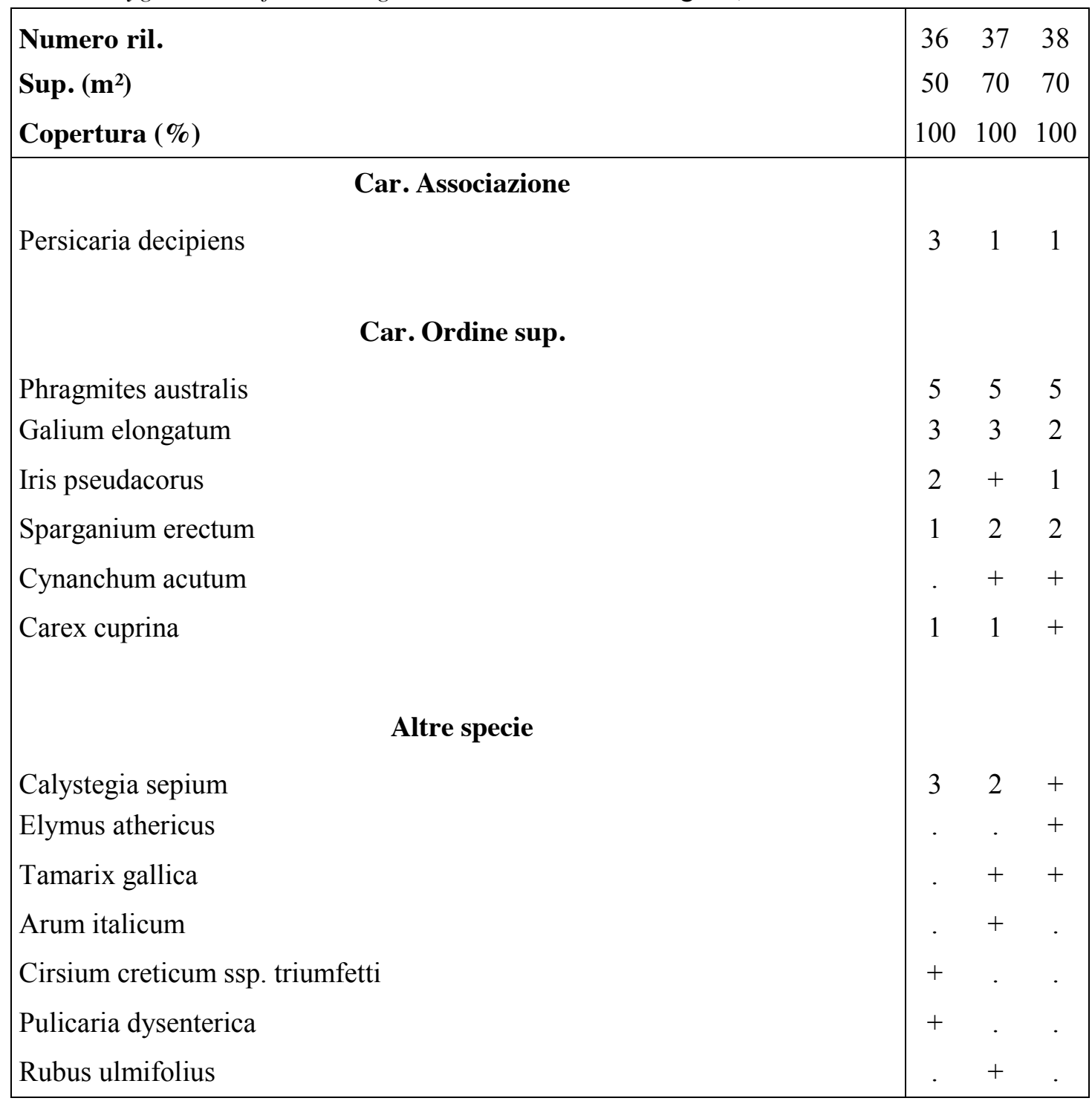


Tab. 10. Phragmitetum communis (Koch 1926) Schmale 1939

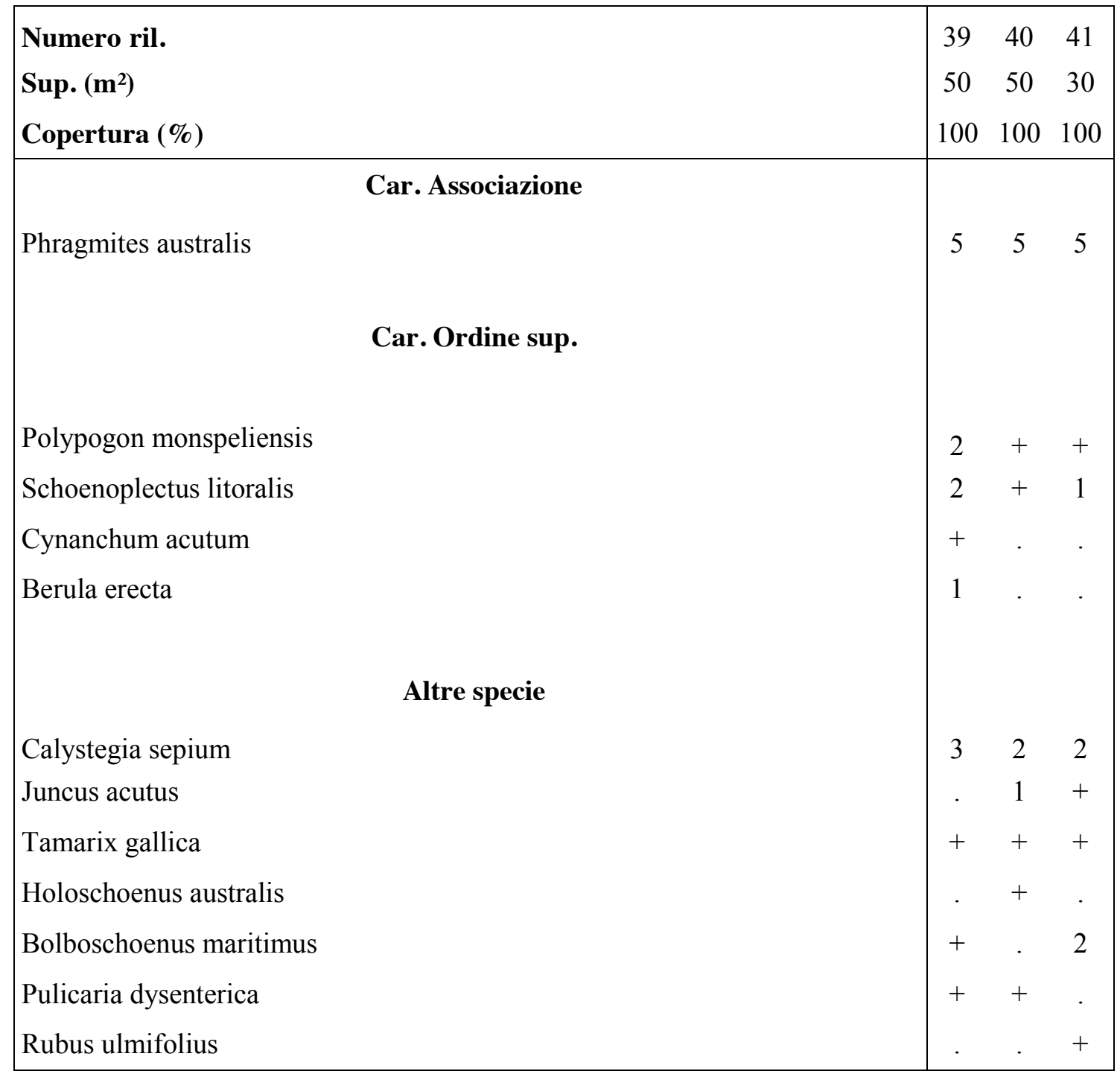


Tab. 11. Typhetum dominguensis Brullo, Minissale \& Spampinato 1994

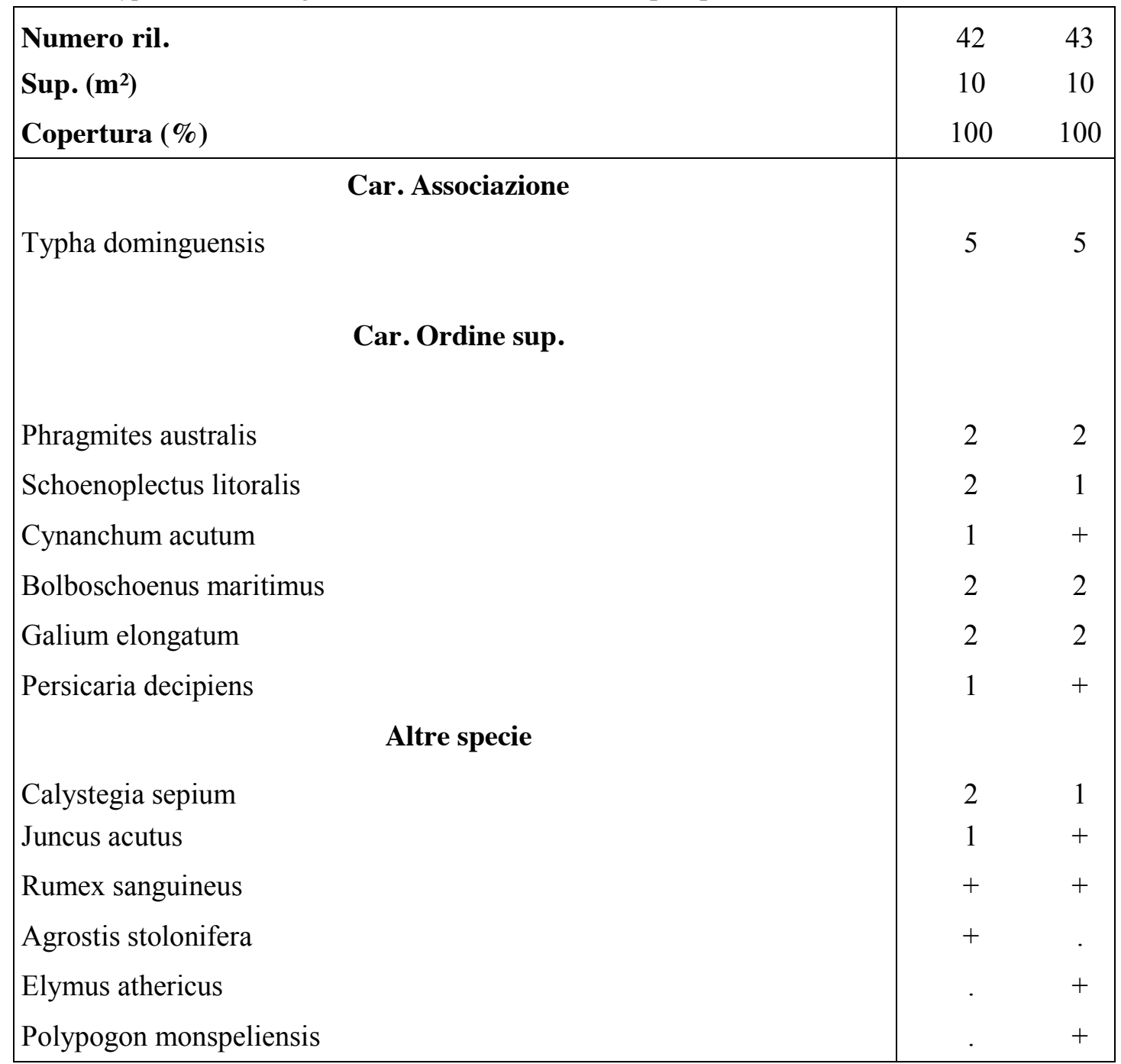


Tab. 12. Typho angustifoliae-Schoenoplectetum tabernaemontani Br.-B1. \& Bolòs 1957

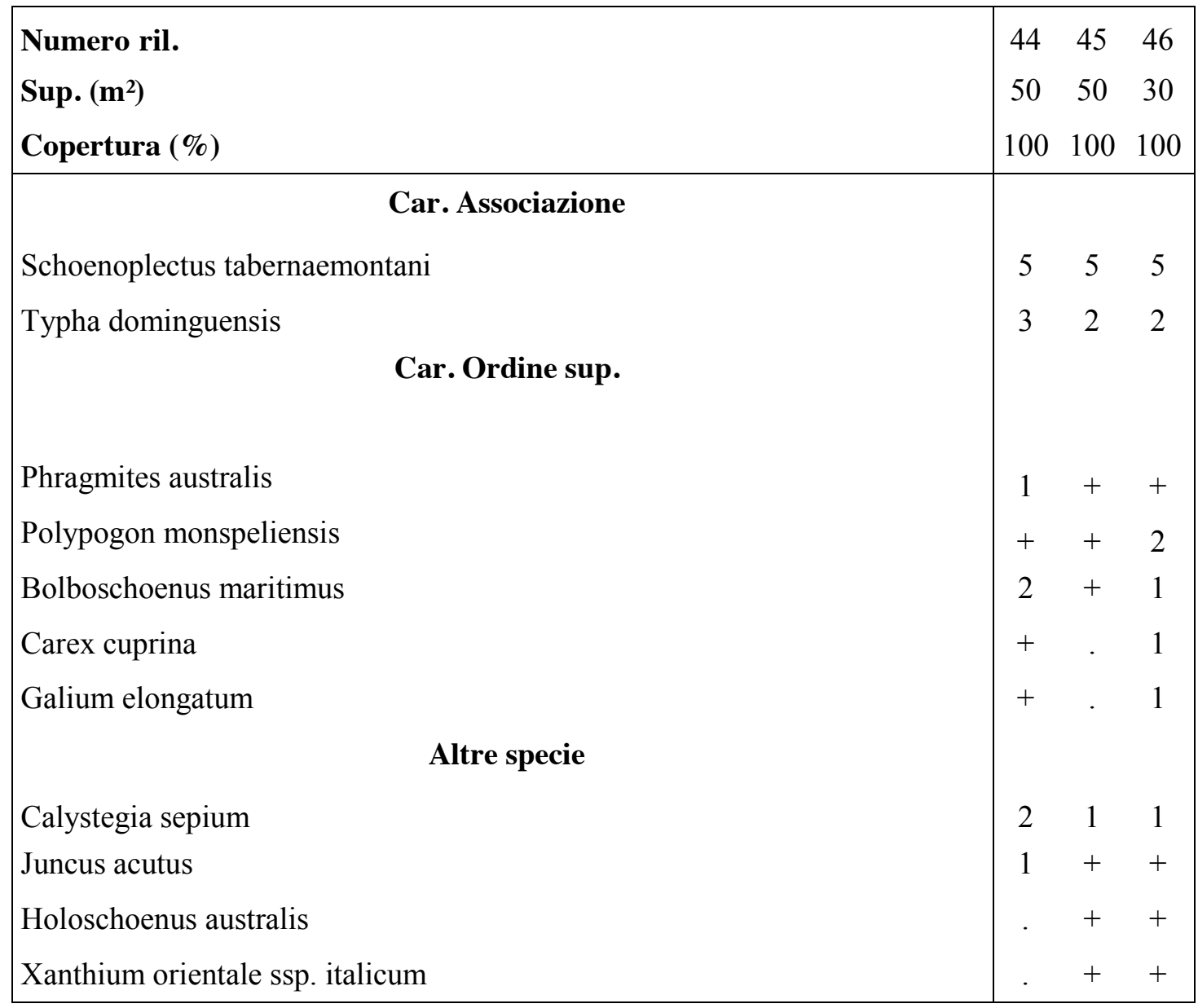


Tab. 13. Sparganietum erecti (Roll. 1938) Philippi 1973

\begin{tabular}{|c|c|c|c|}
\hline $\begin{array}{l}\text { Numero ril. } \\
\text { Sup. }\left(\mathbf{m}^{2}\right) \\
\text { Copertura }(\%)\end{array}$ & $\begin{array}{c}47 \\
50 \\
100\end{array}$ & $\begin{array}{c}48 \\
50 \\
100\end{array}$ & $\begin{array}{c}49 \\
30 \\
100\end{array}$ \\
\hline \multicolumn{4}{|l|}{ Car. Associazione } \\
\hline \multicolumn{3}{|l|}{ Car. Ordine sup. } & 4 \\
\hline Carex cuprina & 1 & + & . \\
\hline Galium elongatum & + & + & . \\
\hline Schoenoplectus tabernaemontani & . & . & + \\
\hline Festuca arundinacea & + & . & . \\
\hline \multicolumn{4}{|l|}{ Altre specie } \\
\hline Apium graveolens & + & . & . \\
\hline Juncus inflexus & . & . & + \\
\hline Potentilla reptans & . & 1 & + \\
\hline Calystegia sylvatica & . & + & + \\
\hline Rumex crispus & . & + & . \\
\hline
\end{tabular}


Tab. 14. Trifolio fragiferi-Cynodontetum dactyli Br.-Bl. \& O. Bolòs 1958

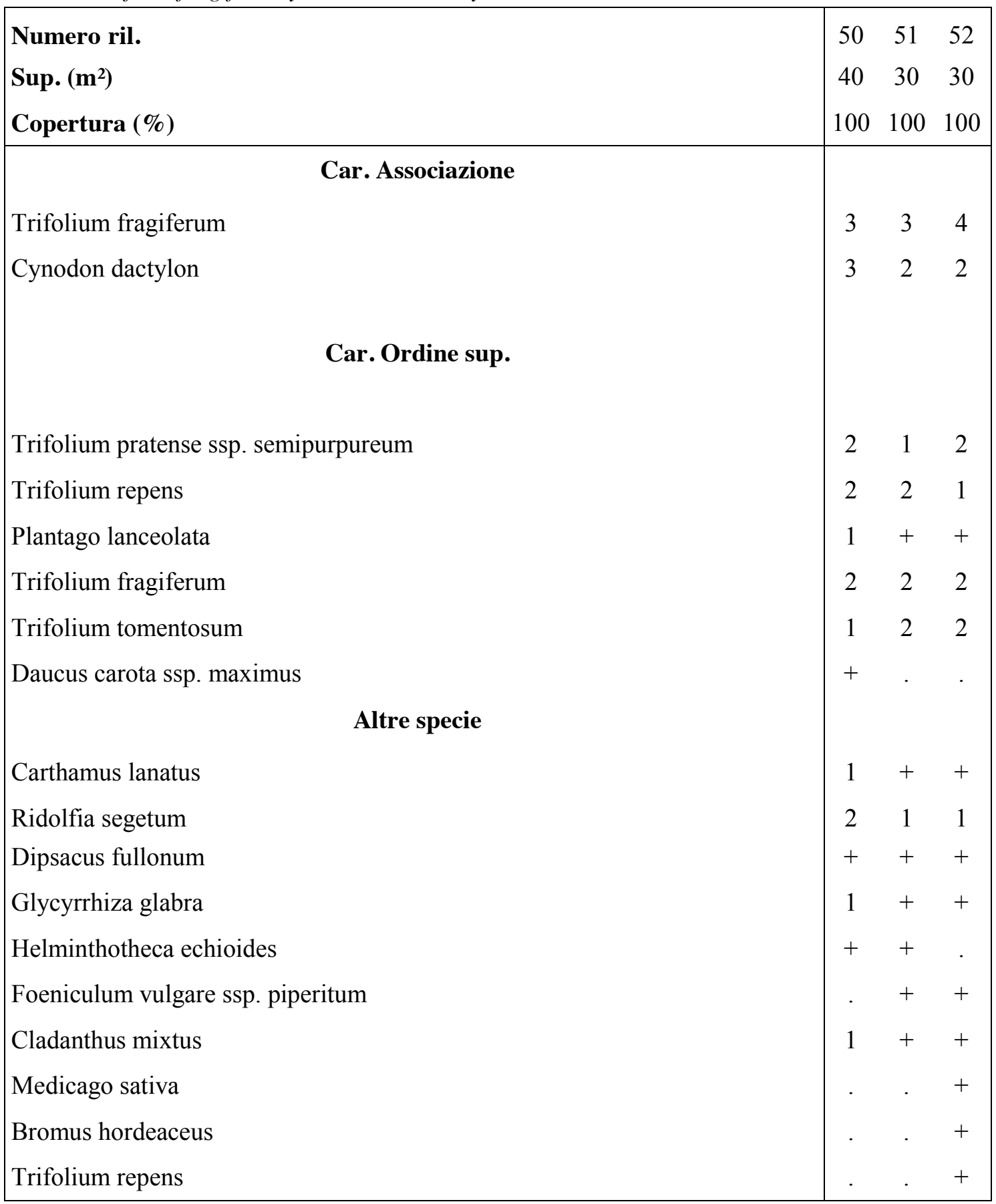


Tab. 15. Tamarici africanae-Viticetum agni-casti Brullo \& Spampinato 1997

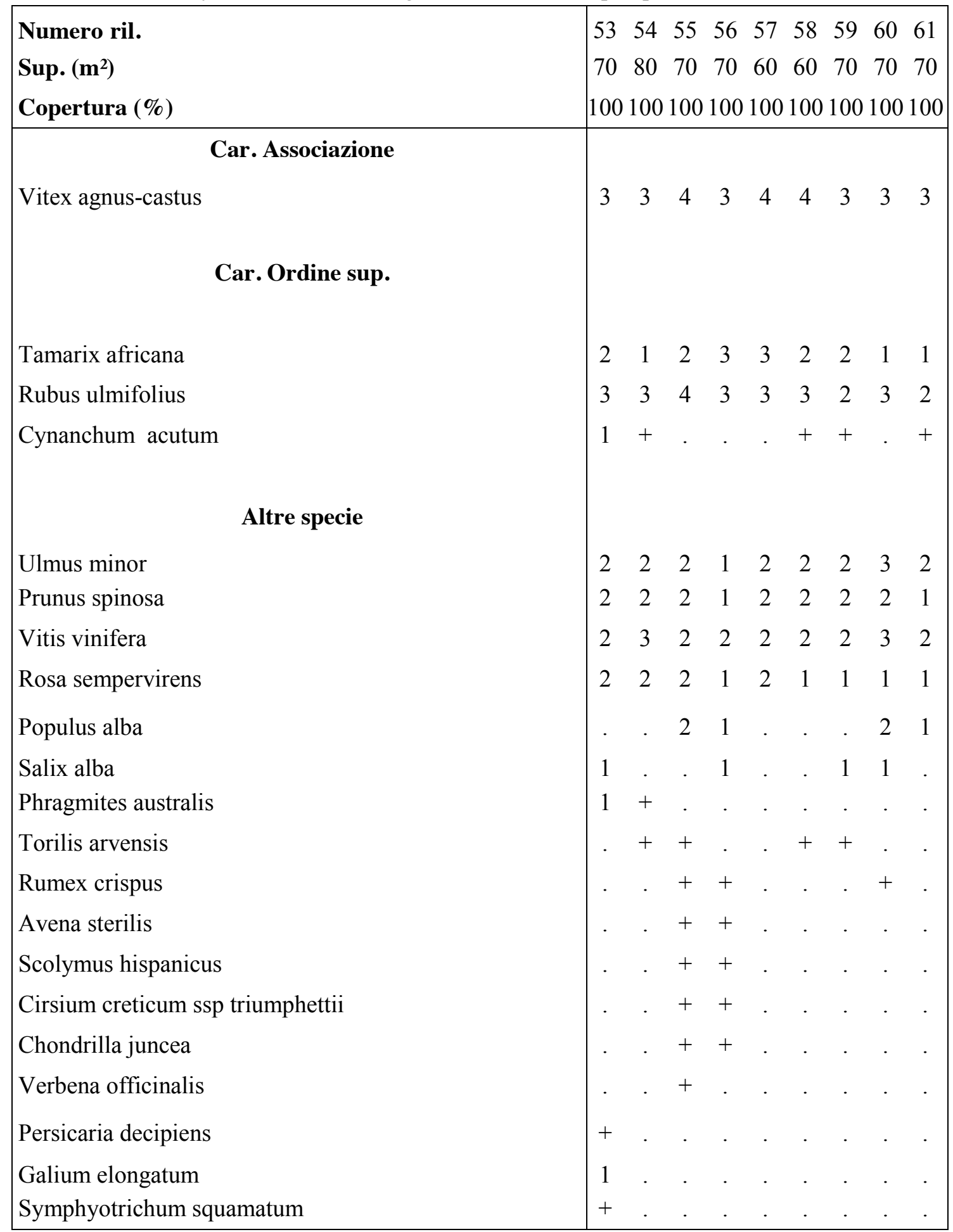


Tab. 16. Salicetum albo-brutiae Brullo \& Spampinato 1997

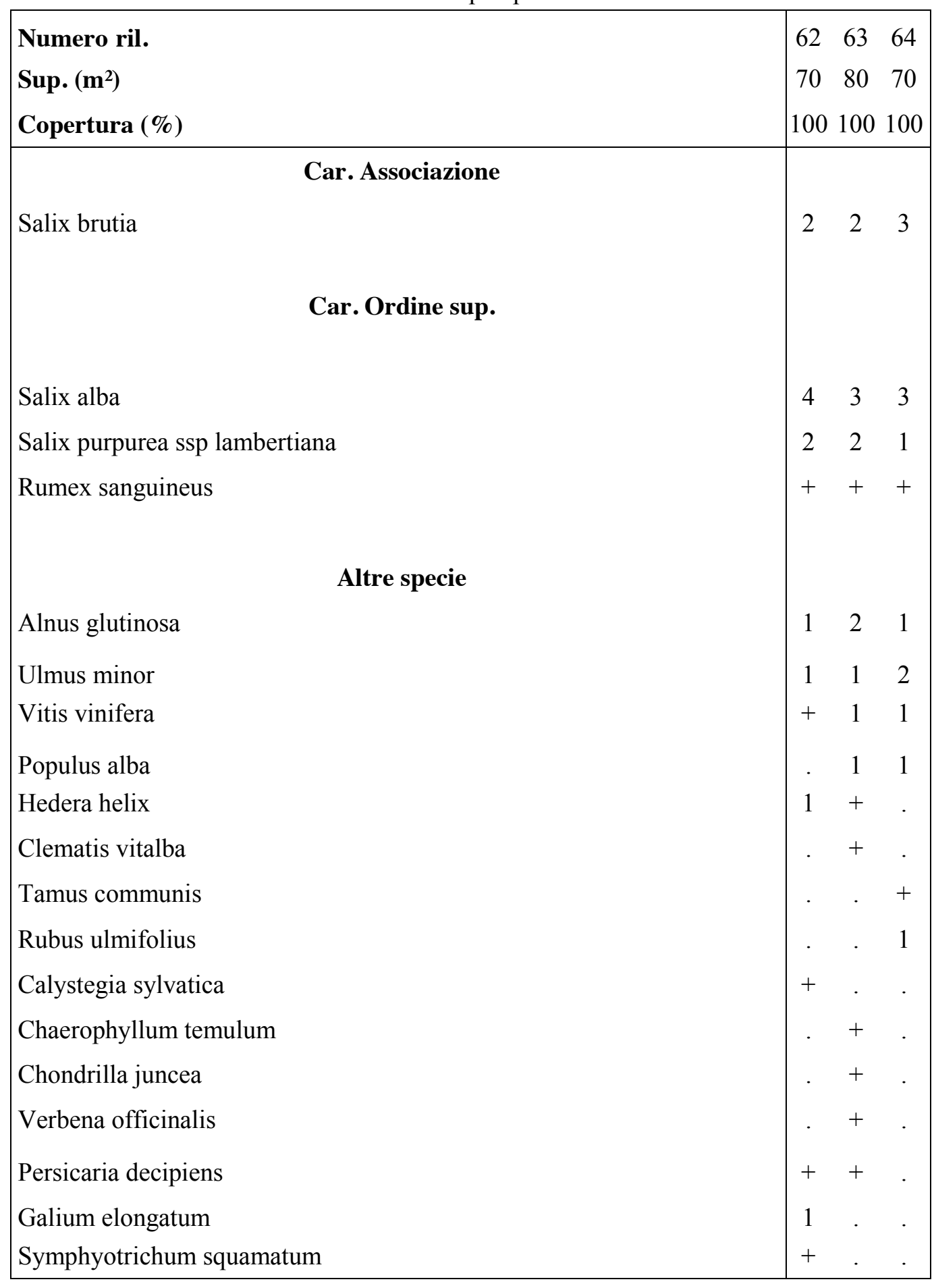


Tab. 17. Carici pendulae-Salicetum cinereae Brullo \& Spampinato 1997

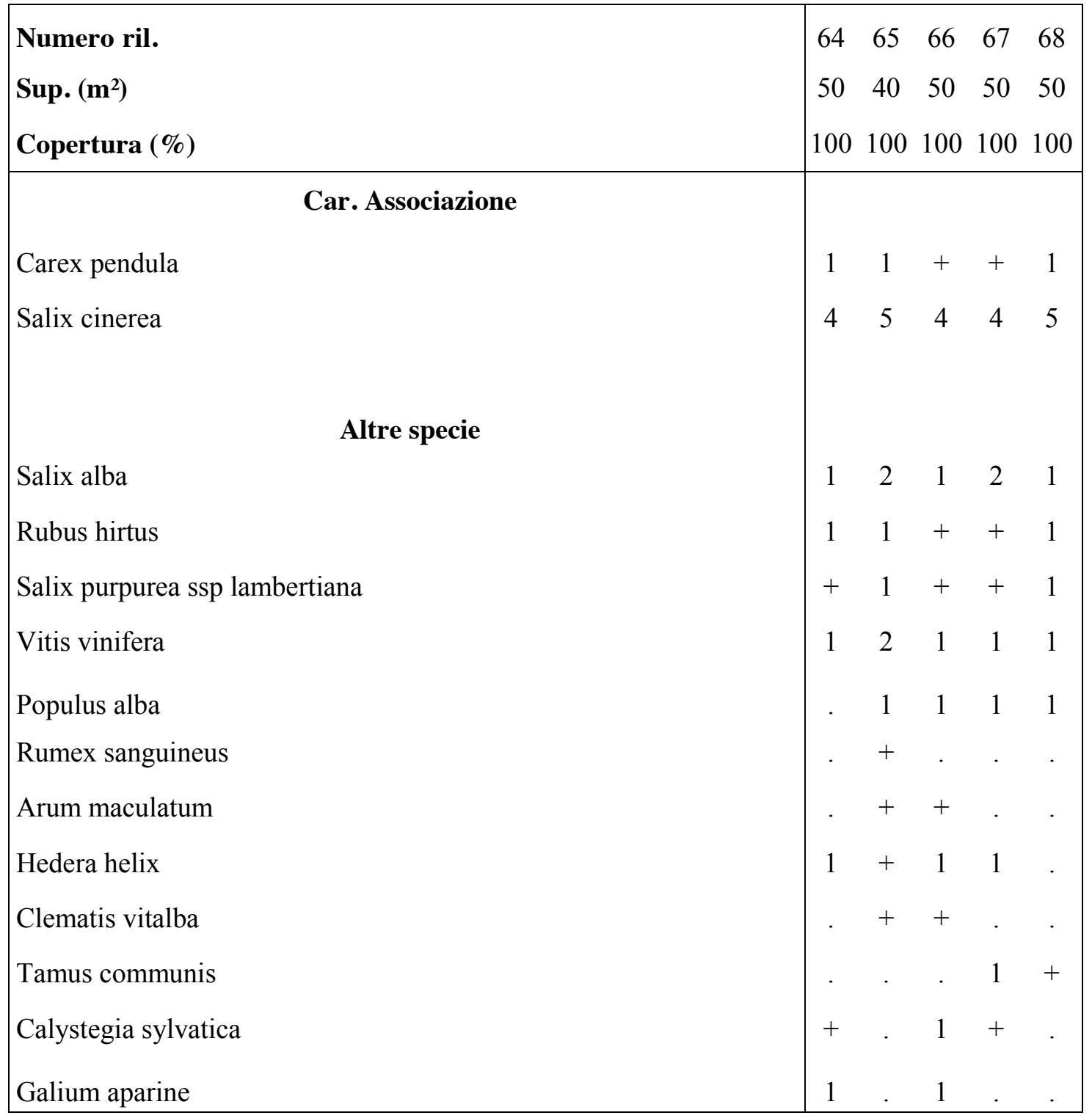


Tab. 18. Angelico sylvestris-Alnetum glutinosae Brullo \& Spampinato 1997 iridetosum pseudacori Brullo \& Spampinato 1997

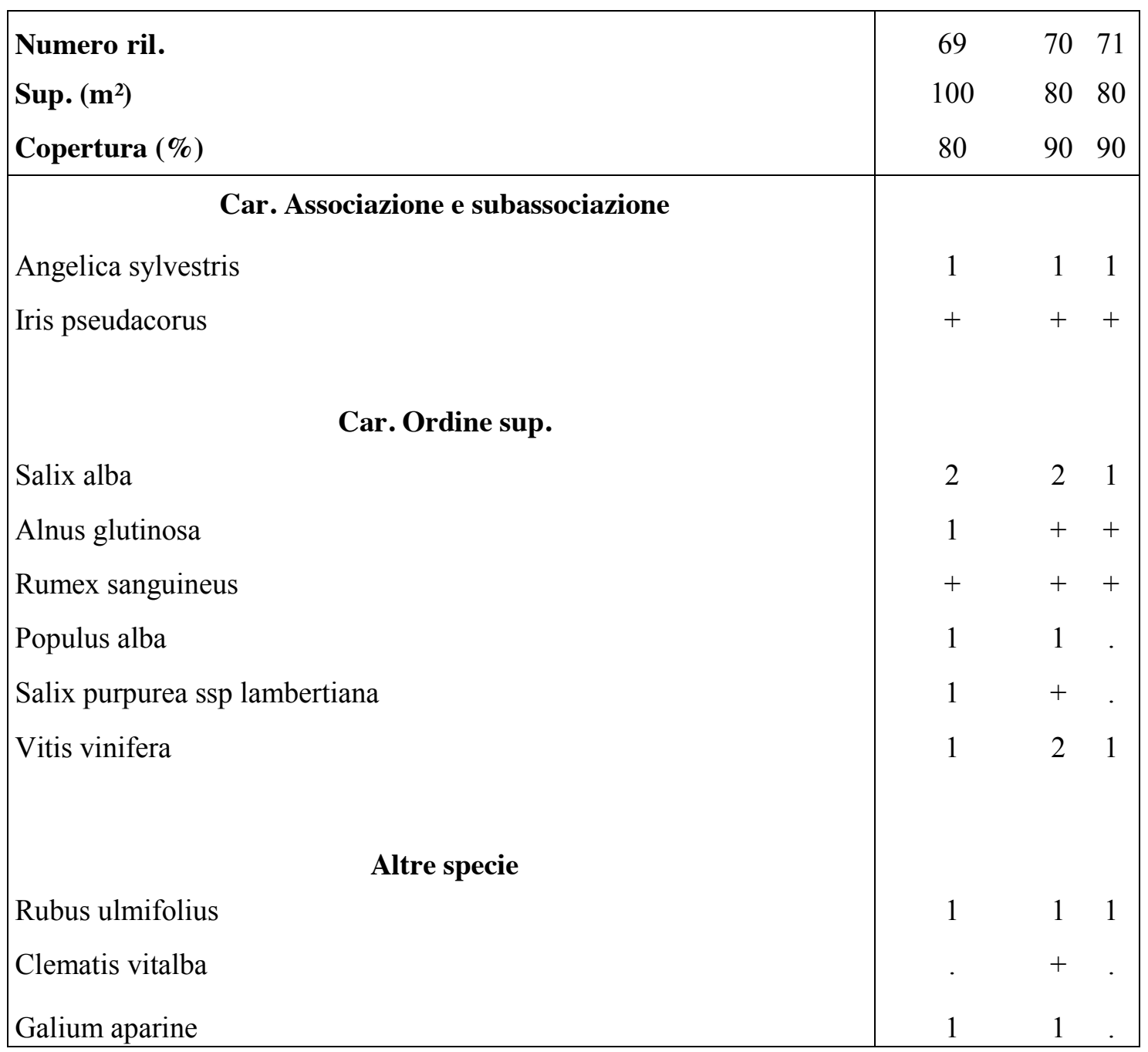


Tab. 19. Clematido viticellae-Populetum albae Brullo \& Spampinato 1997

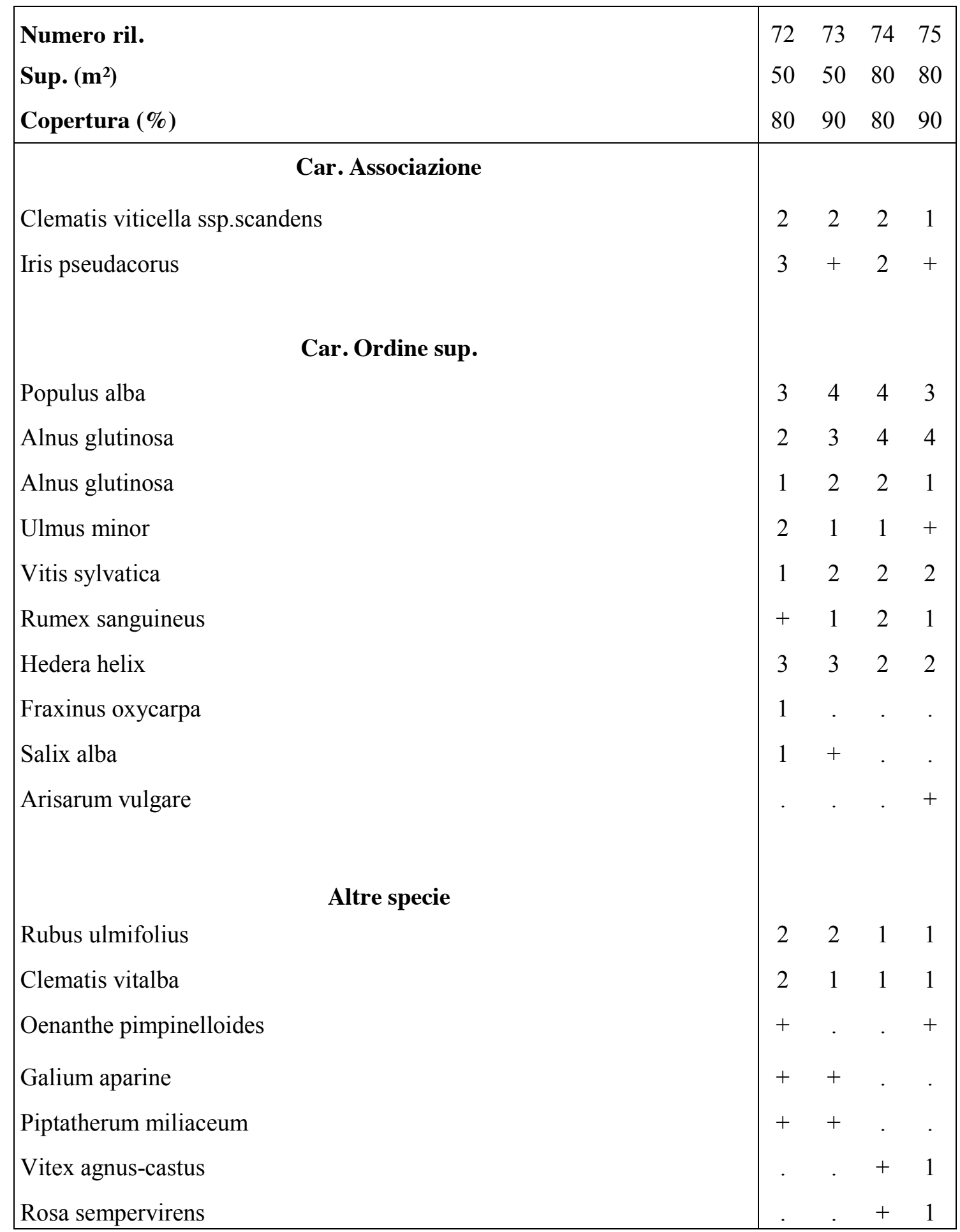


Tab. 20. Fraxino-Quercetum roboris Gellini, Pedrotti \& Venanzoni 1986

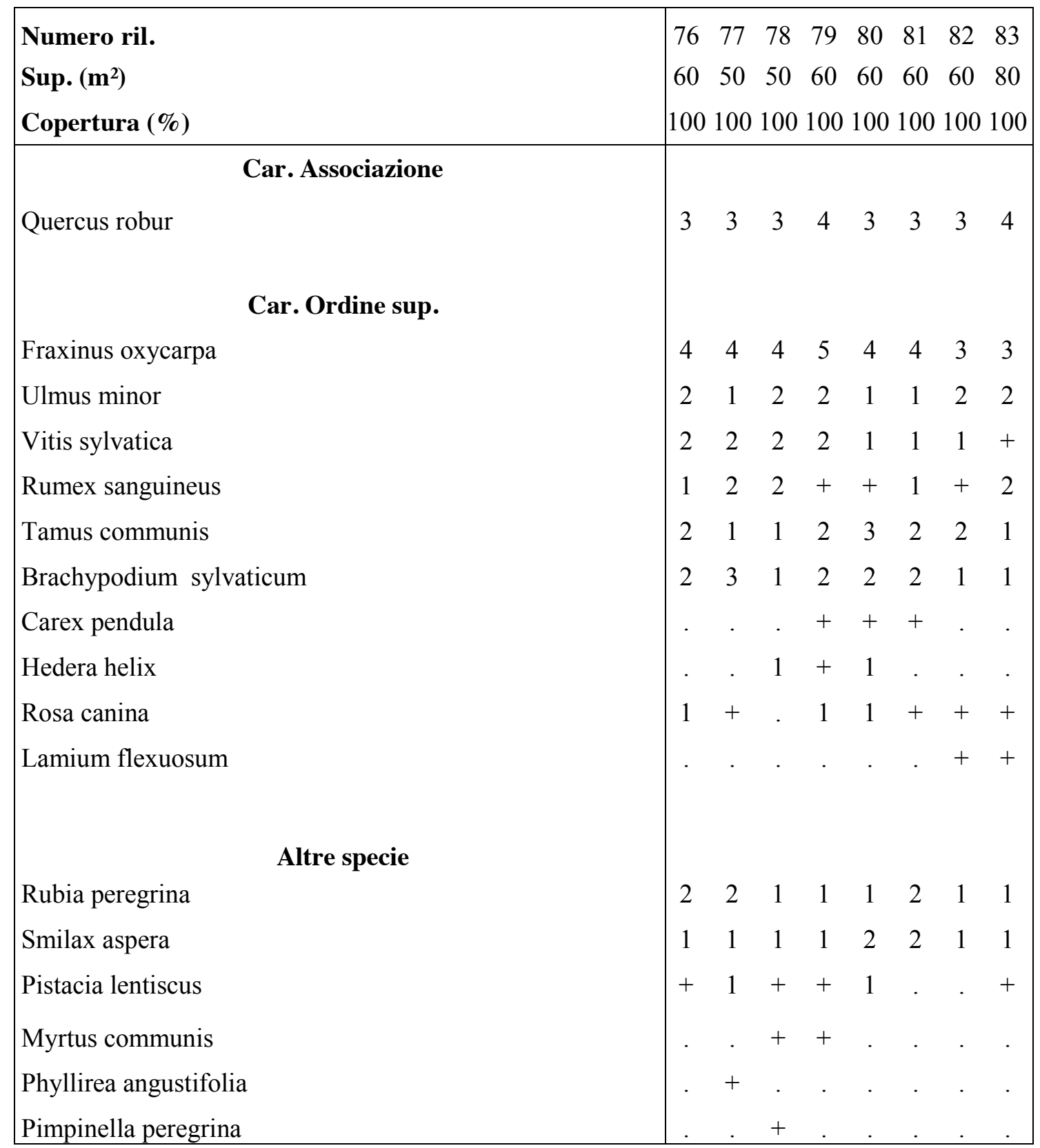


Tab. 21. Asparago acutifolii-Juniperetum macrocarpae (Molinier et R. Molinier 1955) O. Bolòs 1962

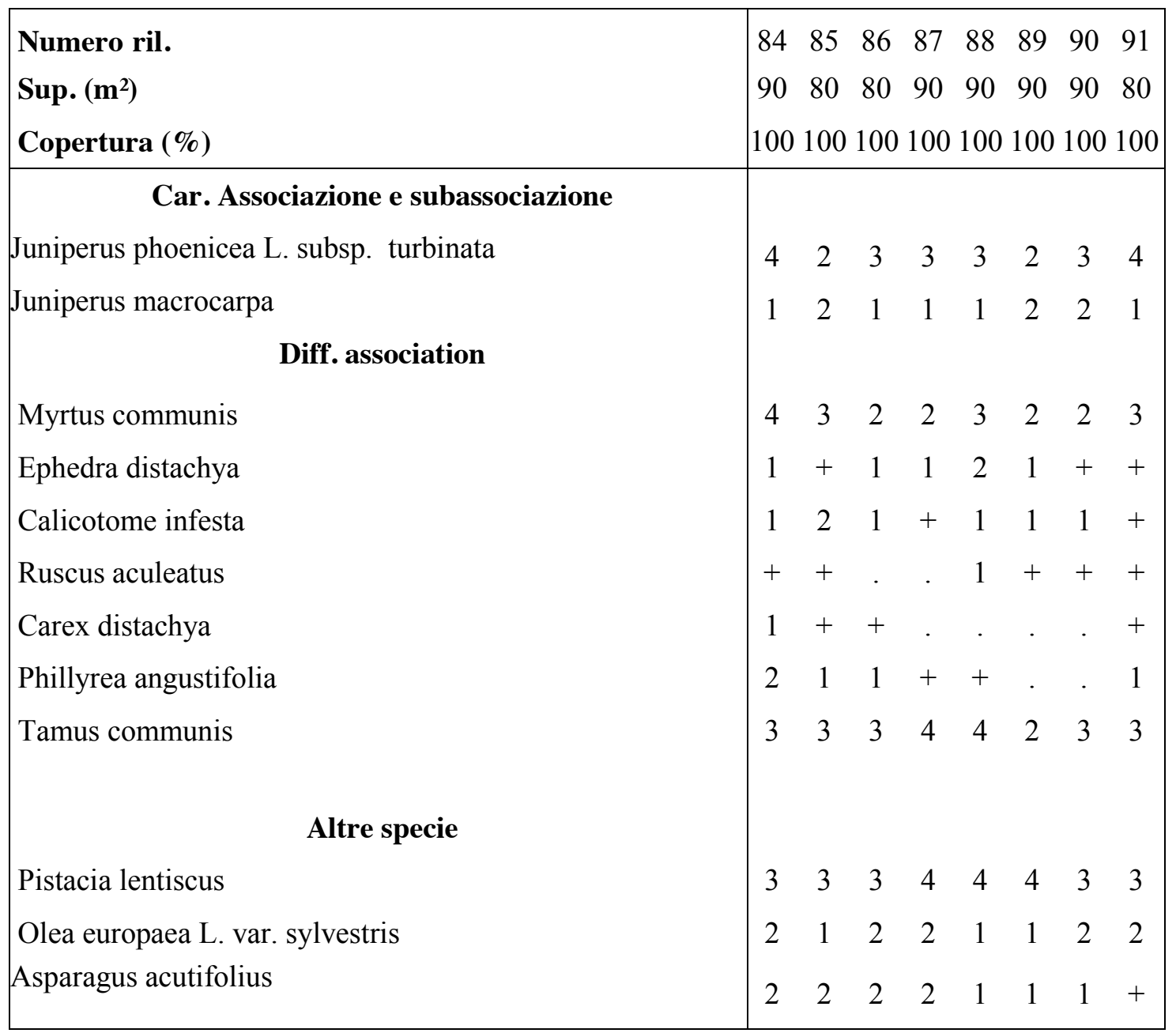


Tab. 22. Myrto-Pistacietum lentisci (Molinier 1954 em. O. Bolos 1962) Rivas-Martinez 1975

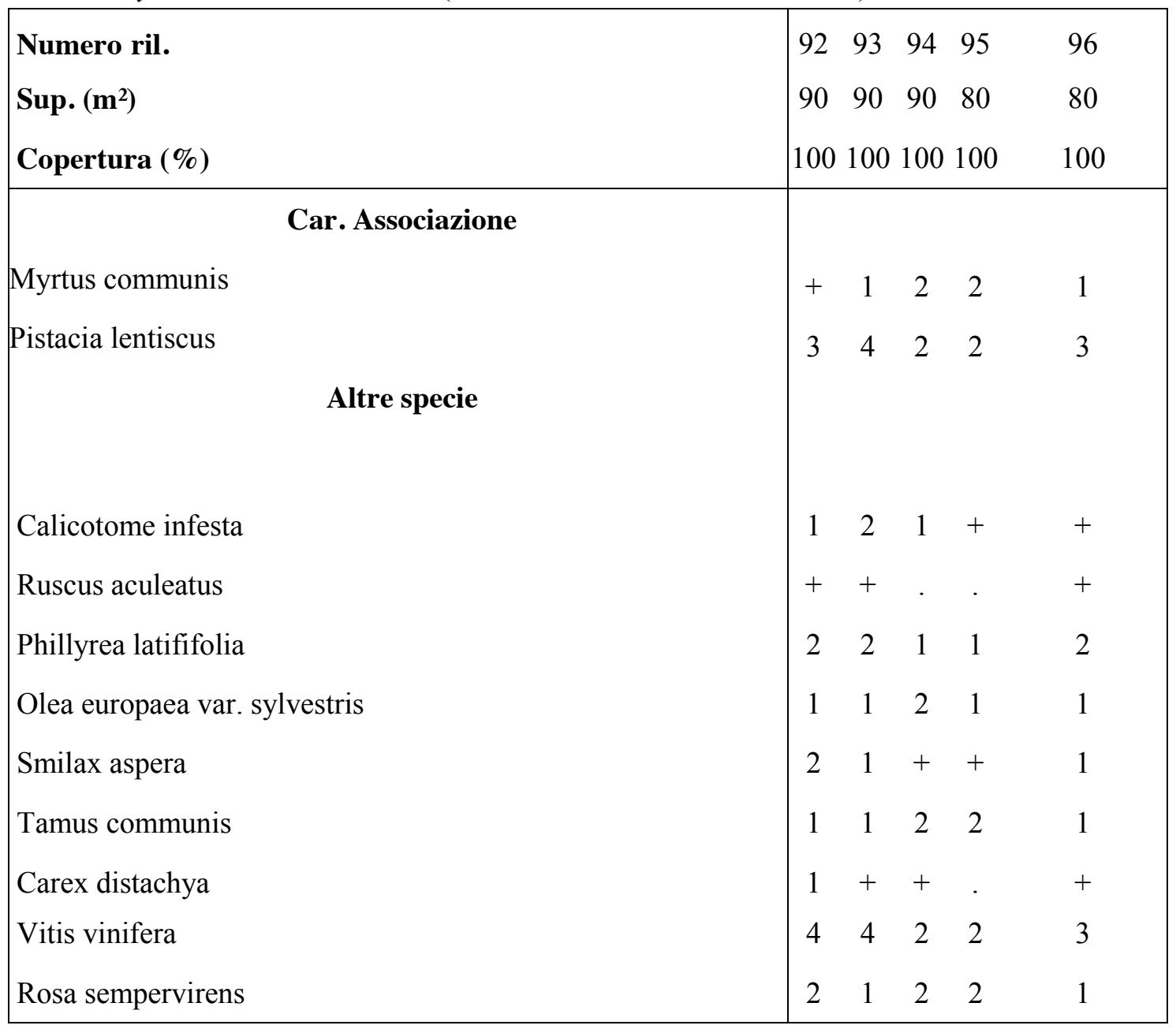


Tab. 23. Centauretum napifoliae Brullo 1983

\begin{tabular}{|c|c|c|c|c|}
\hline \multirow{3}{*}{$\begin{array}{l}\text { Numero ril. } \\
\text { Sup. }\left(\mathbf{m}^{2}\right) \\
\text { Copertura }(\%)\end{array}$} & & 97 & 98 & 99 \\
\hline & & 90 & 90 & 80 \\
\hline & & 90 & 80 & 80 \\
\hline \multirow{3}{*}{ Centaurea napifolia } & r. Associazione & & & \\
\hline & & 3 & 2 & 2 \\
\hline & ar. Ordine sup. & & & \\
\hline \multicolumn{2}{|l|}{ Rostraria cristata } & 2 & 1 & 2 \\
\hline \multicolumn{2}{|l|}{ Glebionis coronaria } & 2 & 2 & 2 \\
\hline \multicolumn{2}{|l|}{ Hordeum murinum } & 2 & 1 & 1 \\
\hline \multicolumn{2}{|l|}{ Melilotus sulcata } & 1 & 1 & 1 \\
\hline \multicolumn{2}{|l|}{ Ammi visnaga } & 1 & 1 & + \\
\hline \multicolumn{2}{|l|}{ Lolium rigidum } & 1 & 1 & 1 \\
\hline \multicolumn{2}{|l|}{ Helminthia echioides } & 1 & 1 & 1 \\
\hline \multicolumn{2}{|l|}{ Ononis mitissima } & + & 1 & 1 \\
\hline \multicolumn{2}{|l|}{ Lactuca serriola } & + & + & + \\
\hline & Altre specie & & & \\
\hline \multicolumn{2}{|l|}{ Ammi majus } & 1 & 2 & 2 \\
\hline \multicolumn{2}{|l|}{ Anagallis arvensis } & + & + & + \\
\hline \multicolumn{2}{|l|}{ Phalaris paradoxa } & 1 & 1 & + \\
\hline \multicolumn{2}{|l|}{ Papaver rhoeas } & + & + & + \\
\hline \multicolumn{2}{|l|}{ Cirsium arvense } & + & + & + \\
\hline
\end{tabular}


Tab. 24. Aggr. a Dasypyrum villosum e Chondrilla juncea

\begin{tabular}{|c|c|c|}
\hline Numero ril. & 100 & 101 \\
\hline Sup. $\left(\mathbf{m}^{2}\right)$ & 50 & 50 \\
\hline Copertura (\%) & 90 & 80 \\
\hline \multicolumn{3}{|l|}{ Car. Associazione } \\
\hline Dasypyrum villosum & 3 & 2 \\
\hline Chondrilla juncea & & \\
\hline \multicolumn{3}{|l|}{ Car. Ordine sup. } \\
\hline Rostraria cristata & 2 & 2 \\
\hline Glebionis coronaria & 2 & 2 \\
\hline Hordeum murinum & 2 & 1 \\
\hline Melilotus sulcata & 1 & 1 \\
\hline Ammi visnaga & 1 & + \\
\hline Lolium rigidum & + & + \\
\hline Erodium malacoides & + & + \\
\hline Helminthia echioides & + & + \\
\hline Medicago polymorpha & + & + \\
\hline Galactites elegans & + & + \\
\hline \multicolumn{3}{|l|}{ Altre specie } \\
\hline Trifolium campestre & 1 & 1 \\
\hline Carthamus lanatus & + & + \\
\hline Anagallis arvensis & + & + \\
\hline Briza maxima & 1 & + \\
\hline Papaver rhoeas & + & + \\
\hline Reichardia picroides & + & + \\
\hline Andryala integrifolia & + & + \\
\hline Trifolium cherleri & + & + \\
\hline
\end{tabular}


Tab. 25. Rimboschimenti a Pinus

\begin{tabular}{|c|c|c|}
\hline Numero ril. & 102 & .103 \\
\hline Sup. $\left(\mathbf{m}^{2}\right)$ & 100 & 100 \\
\hline Copertura strato arboreo $(\%)$ & 90 & 90 \\
\hline Copertura strato erbaceo $(\%)$ & 30 & 30 \\
\hline Pinus halepensis & - & - \\
\hline Myoporum angustifolium & - & - \\
\hline Acacia saligna & - & - \\
\hline Phoenix canariensis & - & - \\
\hline \multicolumn{3}{|l|}{ Car. Tuberarietea guttatae e Alkanno-Malcolmion } \\
\hline Bromus rigidus & 2 & 2 \\
\hline Lagurus ovatus & 2 & 2 \\
\hline Rumex bucephalophorus & 2 & 1 \\
\hline Trifolium campestre & 1 & 1 \\
\hline Medicago littoralis & 1 & + \\
\hline Silene nicaeensis & + & + \\
\hline Cutandia maritima & + & + \\
\hline Helminthia echioides & + & + \\
\hline Medicago polymorpha & + & + \\
\hline Galactites elegans & 1 & + \\
\hline Polycarpon diphyllum & 1 & + \\
\hline Hedypnois rhagadioloides & + & + \\
\hline Tolpis umbellata & 1 & 1 \\
\hline \multicolumn{3}{|l|}{ Altre specie } \\
\hline Trifolium campestre & 1 & 1 \\
\hline Carthamus lanatus & 1 & + \\
\hline Anagallis arvensis & + & + \\
\hline Briza maxima & 1 & + \\
\hline Allium ampeloprasum & + & 1 \\
\hline Papaver rhoeas & . & + \\
\hline Reichardia picroides & + & + \\
\hline Andryala integrifolia & + & + \\
\hline Cyperus kalli & + & + \\
\hline
\end{tabular}


Arcidiacono C., et al.: Naturalità e Vulnerabilità Ambientale nei Siti Natura 2000 che

insistono sui corridoi ecologici del versante jonico calabrese. Caso studio SIC Foce Neto.

Allegato V

ELENCO FLORISTICO 


\section{Elenco delle specie citate nel testo}

Acacia saligna (Labill.) Wendl. fil.

Agrostis stolonifera L.

Agrostis stolonifera subsp. maritima (Lam.) Vasc.

Allium ampeloprasum $\mathrm{L}$.

Alnus glutinosa (L.) Gaertn.

Amaranthus blitoides S. Watson

Amaranthus retroflexus L.

Ammi majus L.

Ammi visnaga (L.) Lam.

Ammophila arenaria (L.) Link ssp. australis (Mabille) Laìnz

Ammophila arenaria subsp. arundinacea (Host) H. Lindb.

Anagallis arvensis $\mathrm{L}$.

Andryala integrifolia $\mathrm{L}$.

Angelica sylvestris $\mathrm{L}$.

Anthemis arvensis L.

Anthemis cotula $\mathrm{L}$.

Apium graveolens $\mathrm{L}$.

Arisarum vulgare O. Targ. Tozz.

Artemisia campestris subsp. variabilis (Ten.) Greuter

Arum italicum Mill.

Arum maculatum L.

Asparagus acutifolius $\mathrm{L}$.

Asphodelus ramosus L.

Atriplex prostrata DC.

Avena barbata Potter

Avena sterilis L.

Berula erecta (Huds.) Coville

Bolboschoenus maritimus (L.) Palla

Brachypodium sylvaticum (Huds.) P. Beauv.

Briza maxima L.

Bromus hordeaceus L.

Bromus madritensis L.

Bromus rigidus Roth

Bromus sterilis L.

Bunias erucago L.

Cachrys pungens Jan

Cakile maritima Scop.

Calendula arvensis $\mathrm{L}$.

Calicotome infesta (C. Presl) Guss.

Calystegia sepium (L.) R. Br.

Calystegia sylvatica (Kit.) Griseb.

Campanula erinus L.

Carex cuprina (Heuff.) A. Kern.

Carex distachya Desf.

Carex extensa Gooden. 
Carex pendula Huds.

Carthamus lanatus L.

Catapodium rigidum (L.) C. E. Hubbard

Centaurea napifolia $\mathrm{L}$.

Chaerophyllum temulum $\mathrm{L}$.

Chenopodium album $\mathrm{L}$.

Chenopodium opulifolium Schrader

Chondrilla juncea $\mathrm{L}$.

Chrozophora tinctoria (L.) A. Juss.

Chrysanthemum coronarium L.

Cirsium arvense (L.) Scop.

Cirsium creticum (Lam.) D'Urv. ssp. triumfetti (Lac.) Werner

Cladanthus mixtus (L.) Chevall.

Clematis vitalba $\mathrm{L}$.

Clematis viticella L. ssp.scandens

Convolvulus arvensis $\mathrm{L}$.

Corynephorus articulatus (Desf.) P. Beauv.

Corynephorus divaricatus (Pourr.) Breistr.

Critesion murinum (L.) A. Love ssp. leporinum (Link) A. Love

Cupressus arizonica Green

Cutandia maritima (L.) Richter

Cynanchum acutum $\mathrm{L}$.

Cynodon dactylon (L.) Pers.

Cyperus kalli (Forsskál) Murb.

Dasypirum villosum (L.) Borbás

Daucus aureus Desf.

Daucus carota L.

Daucus carota subsp. maximus (Desf.) Ball

Delphinium halteratum Sm.

Dioscorea communis (L.) Caddick \& Wilkin

Dipsacus fullonum L.

Dittrichia viscosa (L.) Greuter

Echinophora spinosa L.

Echium arenarium Guss.

Elymus athericus (Link) Kerguélen

Elytrigia juncea (L.) Nevski

Ephedra distachya L.

Epilobium hirsutum L.

Erodium malacoides (L.) L'Her.

Eryngium maritimum $\mathrm{L}$.

Euphorbia exigua L.

Euphorbia falcata L.

Euphorbia helioscopia L.

Euphorbia peplis L.

Euphorbia terracina L.

Fallopia convolvulus (L.) Á Löve

Ferula communis L. 
Festuca arundinacea Schreb.

Filago pyramidata $\mathrm{L}$.

Foeniculum vulgare subsp. piperitum (Ucria) Cout.

Frankenia pulverulenta L.

Galactites elegans (All.) Soldano

Galium aparine L.

Galium elongatum C. Presl

Gladiolus communis L.

Glaucium flavum Crantz

Glebionis coronaria (L.) Spach

Glycyrrhiza glabra L.

Hedera helix $\mathrm{L}$.

Hedypnois cretica (L.) Dum.-Courset

Hedypnois rhagadioloides (L.) F.G. Schmidt

Helminthotheca echioides (L.) Holub

Holoschoenus australis (L.) Rchb.

Hordeum marinum Huds.

Hordeum murinum L.

Hypecoum procumbens $\mathrm{L}$.

Hypochaeris achyrophorus $\mathrm{L}$.

Iris pseudacorus L.

Juncus acutus L. ssp. acutus

Juncus inflexus L.

Juncus subulatus Forssk.

Juniperus oxycedrus ssp. macrocarpa (Sm.) Ball

Juniperus phoenicea L. subsp. turbinata

Kickxia spuria (L.) Dumort. ssp. integrifolia (Brot.) R. Fern.

Lactuca serriola $\mathrm{L}$.

Lagurus ovatus L. ssp. ovatus

Lamium flexuosum Ten.

Lobularia maritima (L.) Desv.

Lolium rigidum Gaudin

Lotus creticus $\mathrm{L}$.

Lotus tenuis Willd.

Matthiola sinuata (L.) R. Br.

Medicago littoralis Loisel

Medicago marina L.

Medicago minima (L.) Bartal.

Medicago polymorpha $\mathrm{L}$.

Medicago rigidula (L.) All.

Medicago sativa $\mathrm{L}$.

Melilotus sulcatus Desf.

Mentha pulegium L.

Myoporum angustifolium Benth.

Myoporum tenuifolium Forster

Myrtus communis L.

Nigella arvensis L. 
Oenanthe pimpinelloides L.

Olea europaea L. ssp. oleaster (Hoffmanns. \& Link) Negodi

Olea europaea L. var. sylvestris

Onobrychis caput-galli Lam.

Ononis mitissima L.

Ononis variegata $\mathrm{L}$.

Onopordum illyricum L.

Opuntia ficus-indica (L.) Mill.

Orobanche minor Sm.

Oryzopsis coerulescens (Desf.) Richter

Oryzopsis miliacea (L.) Asch. et Schweinf. ssp. thomasii (Duby) Pign.

Otanthus maritimus (L.) Hoffmanns et Link

Pancratium maritimum L.

Papaver rhoeas L.

Persicaria decipiens (R. Br.) K. L. Wilson

Phalaris brachystachys Link

Phalaris paradoxa $\mathrm{L}$.

Phillyrea angustifolia $\mathrm{L}$.

Phillyrea latifolia $\mathrm{L}$.

Phoenix canariensis Chabaud

Phragmites australis (Cav.) Steud.

Phyllirea angustifolia $\mathrm{L}$.

Pimpinella peregrina L.

Pinus halepensis Miller

Pinus pinea $\mathrm{L}$.

Piptatherum miliaceum (L.) Coss.

Pistacia lentiscus L.

Plantago lagopus L.

Plantago lanceolata L.

Polycarpon diphyllum Cav.

Polygonum aviculare L.

Polygonum maritimum L.

Polypogon monspeliensis (L.) Desf.

Populus alba L.

Portulaca oleracea L.

Potentilla reptans $\mathrm{L}$.

Prunus spinosa $\mathrm{L}$.

Pseudorlaya pumila (L.) Grande

Pulicaria dysenterica (L.) Bernh.

Quercus robur L.

Ranunculus ficaria $\mathrm{L}$.

Reichardia picroides (L.) Roth

Reseda alba $\mathrm{L}$.

Ridolfia segetum Moris

Rosa canina $\mathrm{L}$.

Rosa sempervirens $\mathrm{L}$.

Rostraria cristata (L.) Tzvelev 
Rubia peregrina $\mathrm{L}$.

Rubus hirtus Waldst. \& Kit.

Rubus ulmifolius Schott

Rumex bucephalophorus L.

Rumex crispus L.

Rumex pulcher $\mathrm{L}$.

Rumex sanguineus $\mathrm{L}$.

Ruscus aculeatus L.

Salicornia patula Duval-Jouve

Salix alba L.

Salix brutia Brullo \& Spamp.

Salix cinerea L.

Salix purpurea subsp. lambertiana (Sm.) Rech. f.

Salsola kali L.

Salsola soda L.

Schoenoplectus litoralis (Schrad.) Palla

Schoenoplectus tabernaemontani (C. C. Gmel.) Palla

Scolymus hispanicus L.

Silene coeli-rosa (L.) Godron

Silene colorata Poiret

Silene nicaeensis All.

Sinapis arvensis L.

Sisymbrium officinale (L.) Scop.

Smilax aspera L.

Solanum nigrum $\mathrm{L}$.

Sparganium erectum $\mathrm{L}$.

Spergularia marina (L.) Griseb.

Spergularia media (L.) C. Presl

Spergularia salina J. \& C. Presl

Sporobolus pungens (Schreber) Kunth

Stipa capensis Thumb.

Suaeda maritima (L.) Dumort.

Symphyotrichum squamatum

Symphyotrichum squamatum (Spreng.) G. L. Nesom

Tamarix africana Poiret

Tamarix gallica L.

Tolpis umbellata Bertol.

Torilis arvensis (Hudson) Link

Tragopogon porrifolius $\mathrm{L}$.

Tribulus terrestris L.

Trifolium campestre Schreber

Trifolium cherleri $\mathrm{L}$.

Trifolium fragiferum $\mathrm{L}$.

Trifolium hybridum $\mathrm{L}$.

Trifolium pratense ssp. semipurpureum (Strobl) Pignatti

Trifolium repens $\mathrm{L}$.

Trifolium resupinatum $\mathrm{L}$. 
Arcidiacono C., et al.: Naturalità e Vulnerabilità Ambientale nei Siti Natura 2000 che insistono sui corridoi ecologici del versante jonico calabrese. Caso studio SIC Foce Neto.

Trifolium scabrum L.

Trifolium tomentosum $\mathrm{L}$.

Tripolium pannonicum (Jacq.) Dobrocz.

Typha domingensis (Pers.) Steud.

Ulmus minor Hudson

Verbascum sinuatum L.Samp.

Verbena officinalis L.

Vitex agnus-castus $\mathrm{L}$.

Vitis vinifera $\mathrm{L}$.

Vulpia fasciculate (Forssk.) Samp,

Vulpia membranacea (L.) Dumort.

Vulpia myuros (L.) Gmelin

Xanthium italicum Moretti 
Allegato VI

ICONOGRAFIA 


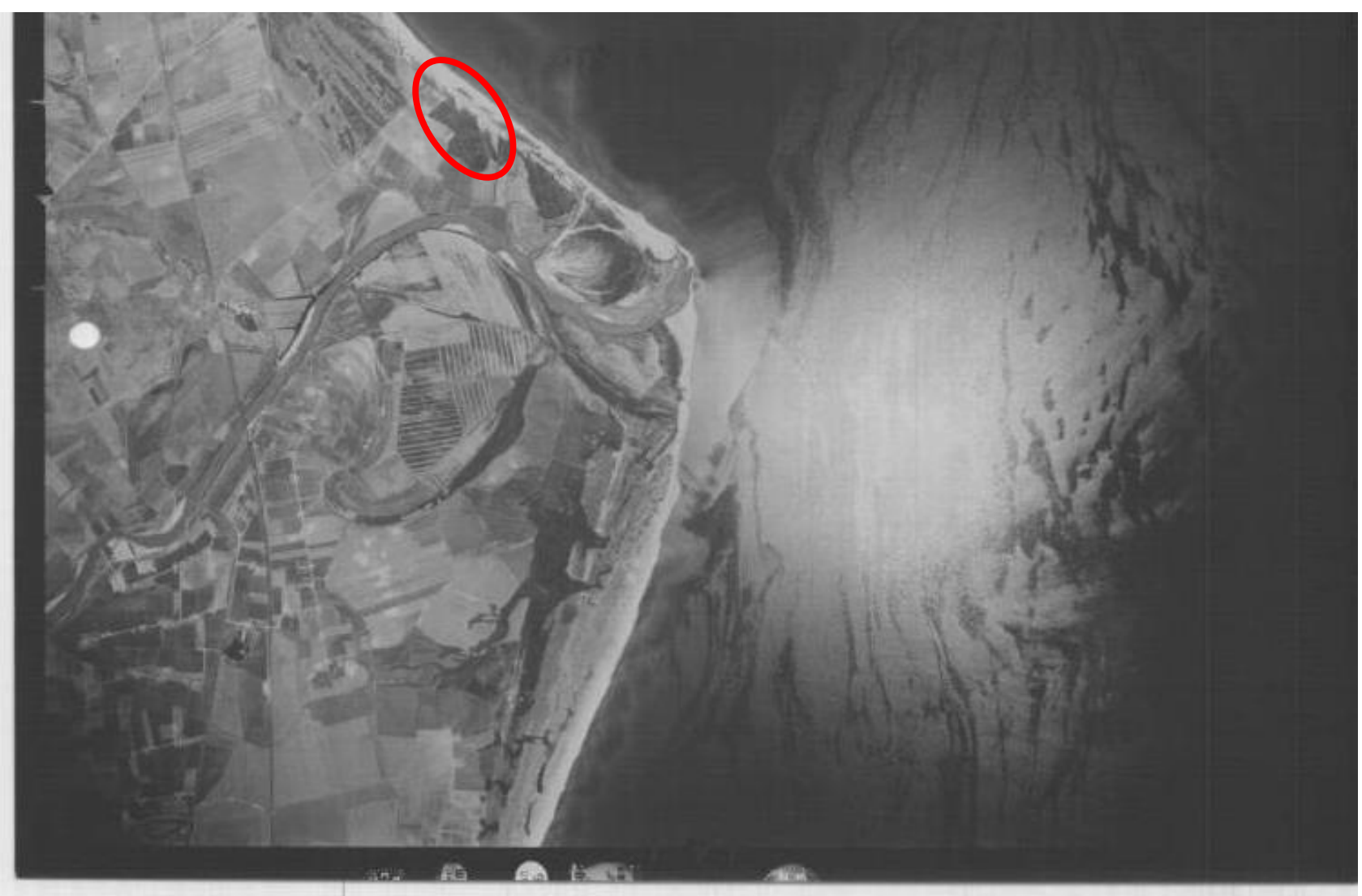

Foto storica. (C) 2015 Istituto Geografico Militare 1955-06-22. Foce del Fiume Neto e aree limitrofe

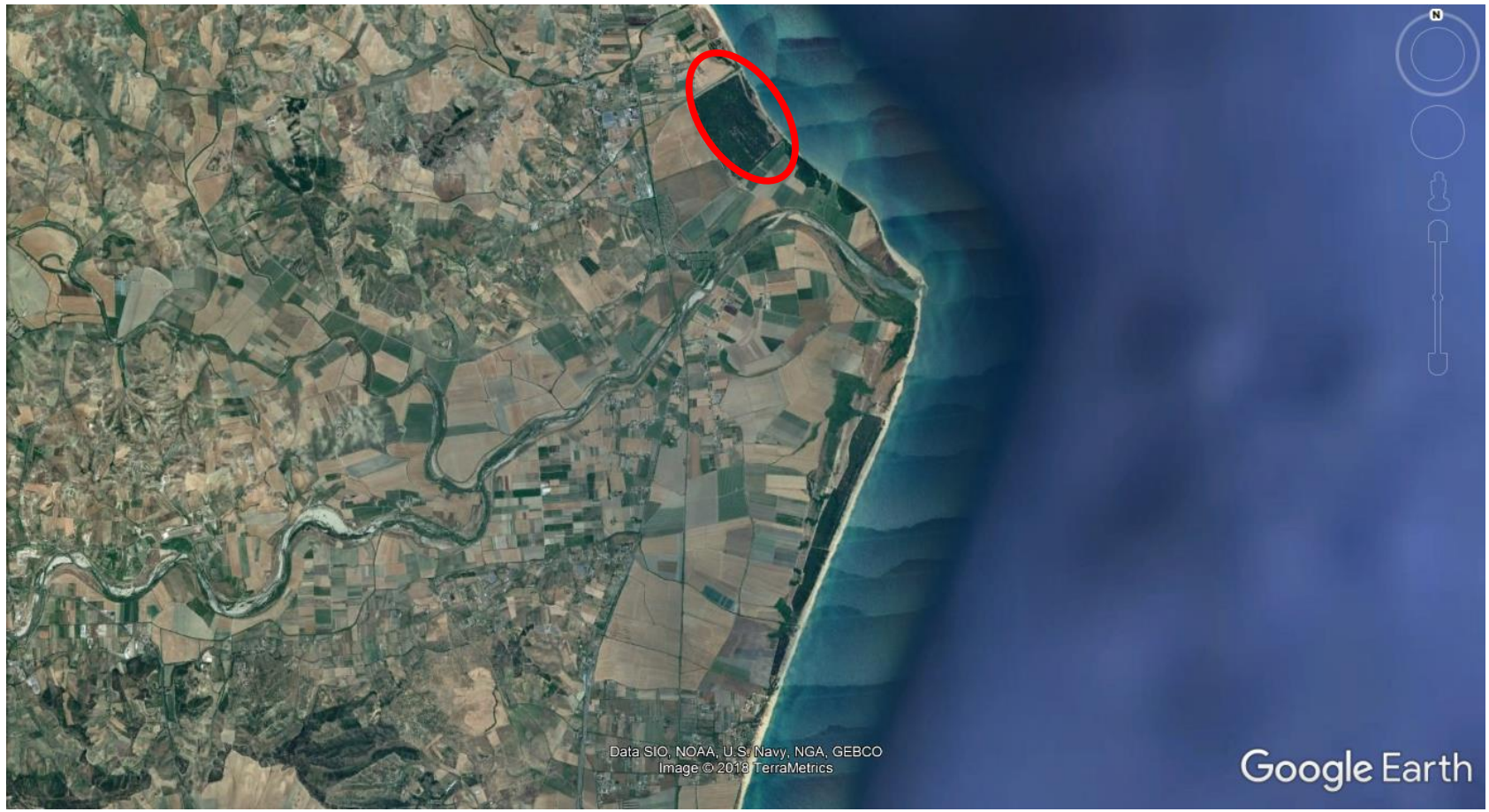

Foto satellitare attuale. In evidenza le aree con rimboschimenti 


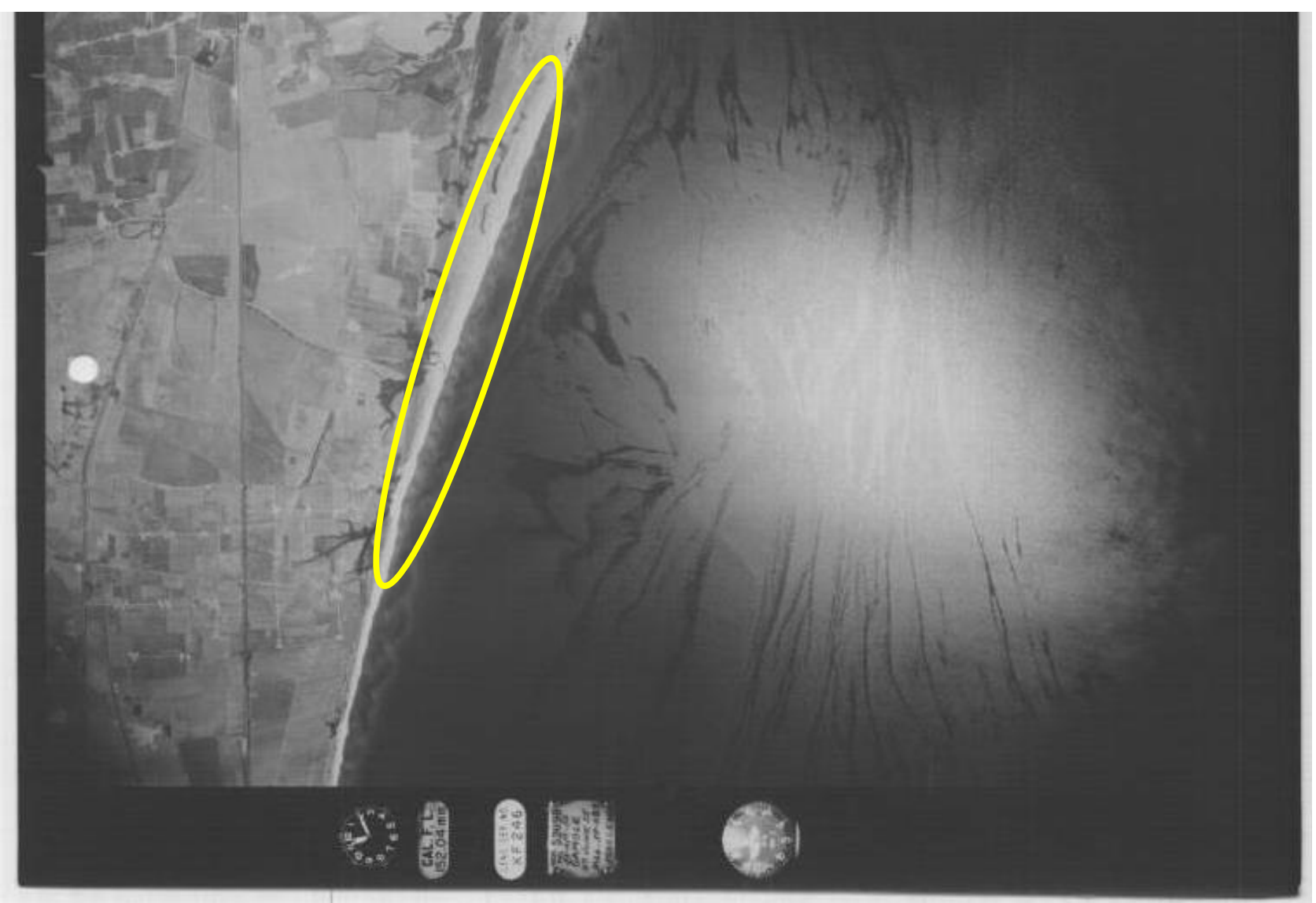

Foto storica. (C) 2015 Istituto Geografico Militare 1943-01-23.

Si evince l'assenza del rimboschimento a Pinus sp pl ed Eucaliptus sp pl

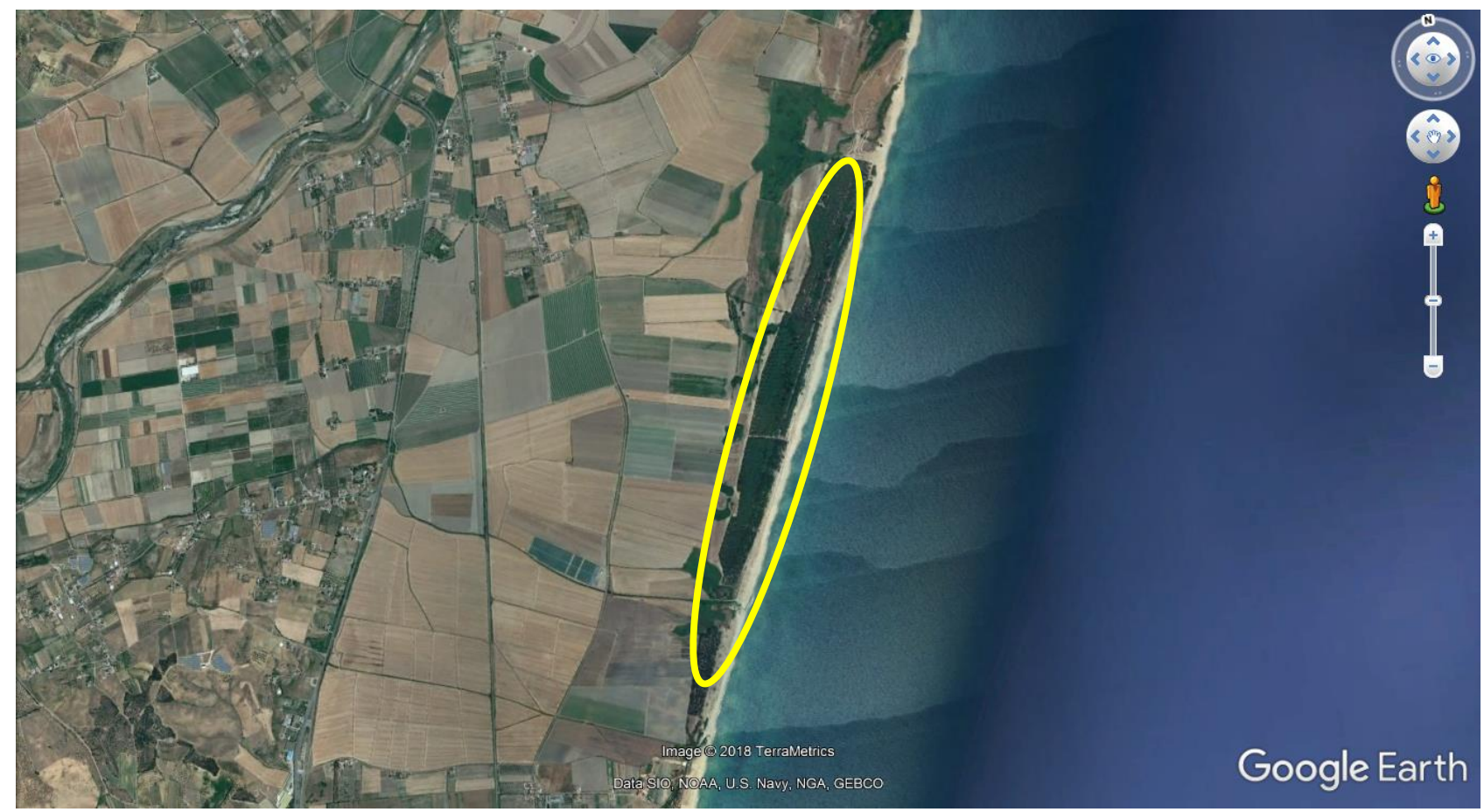

Foto satellitare attuale. In evidenza le aree con rimboschimenti 


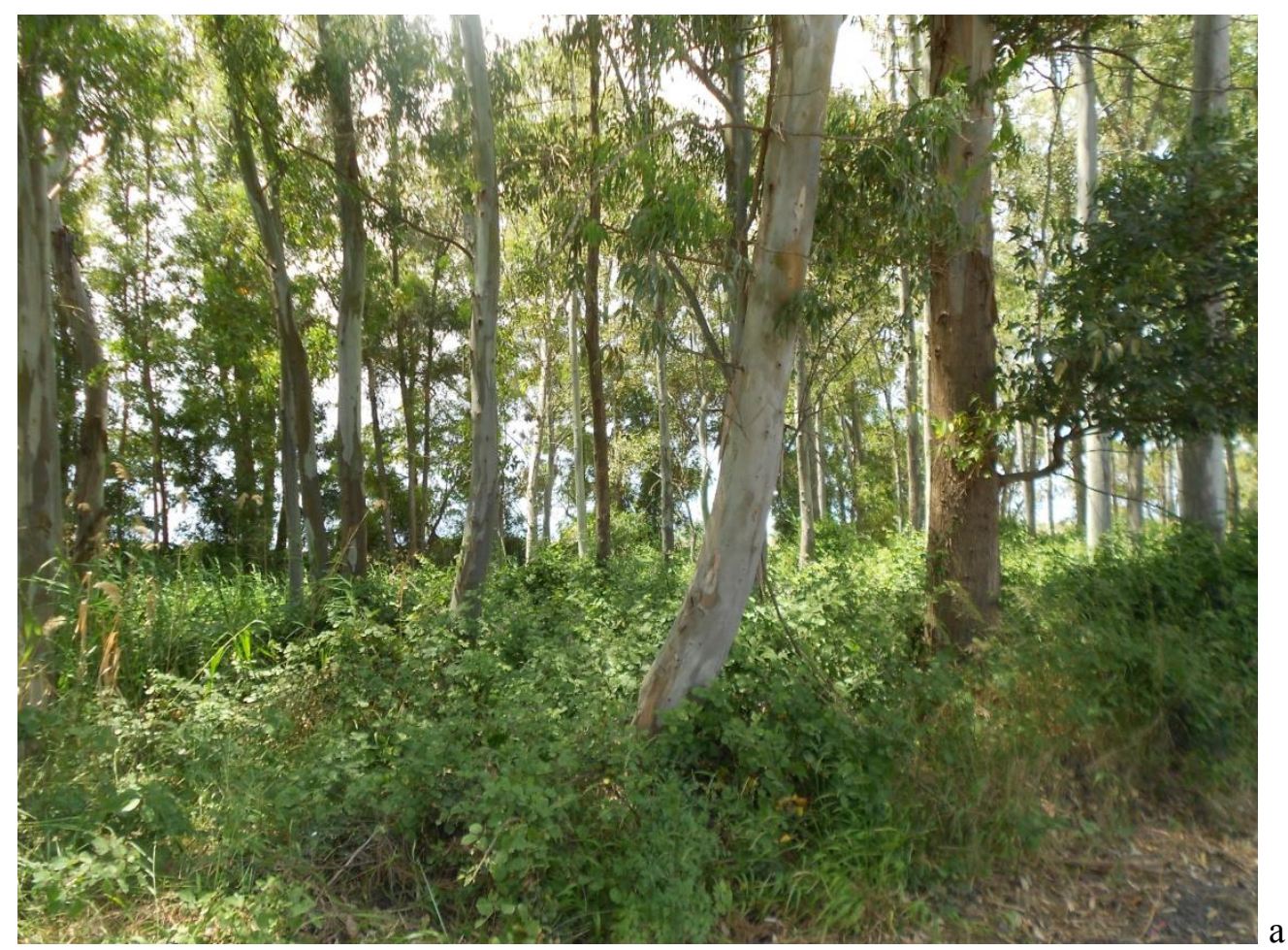

Rimboschimento ad Eucaliptus sp pl.

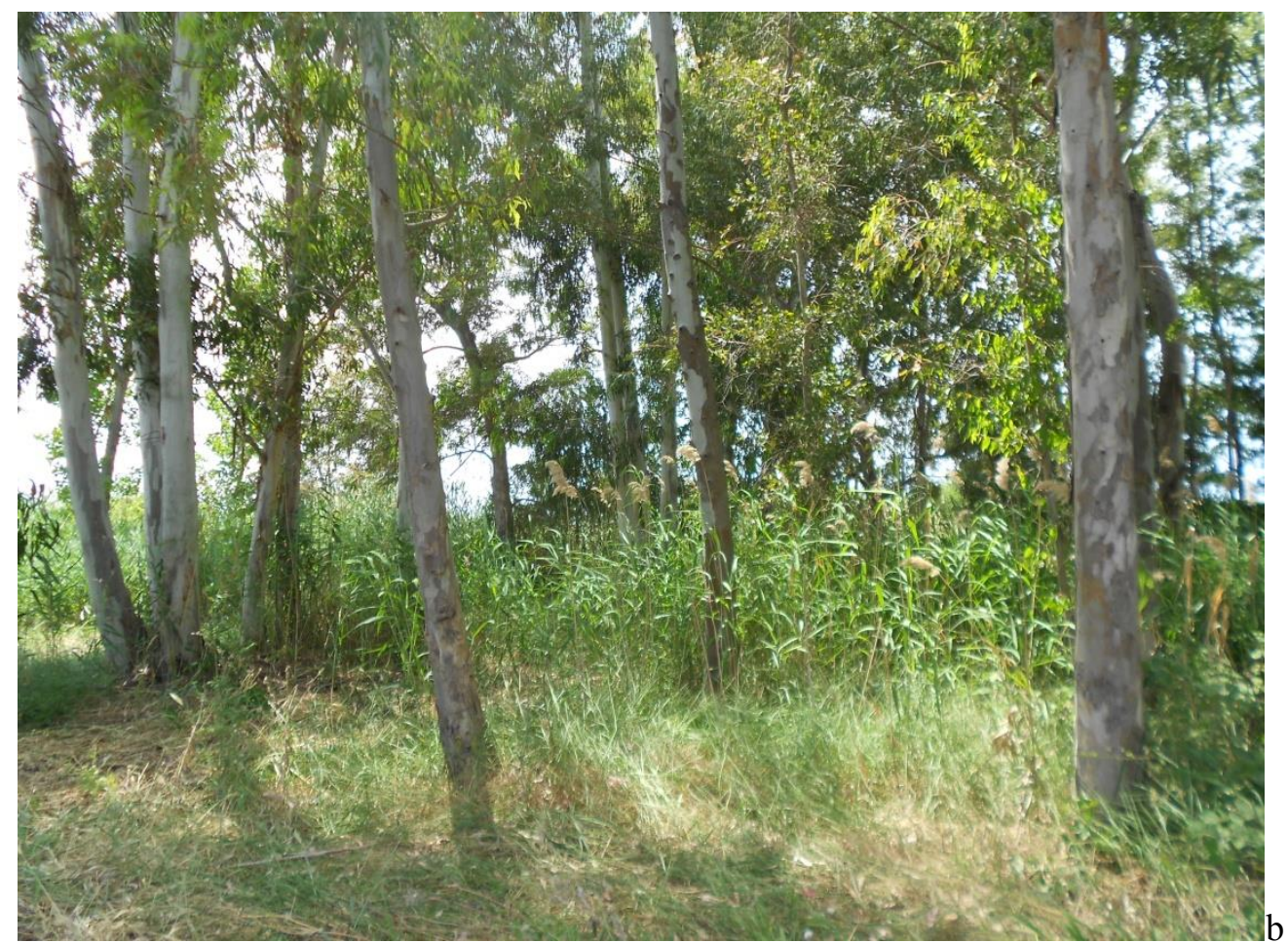

Rimboschimento ad Eucaliptus sp pl. La vegetazione non si esprime secondo le potenzialità del sito $(\mathrm{a}, \mathrm{b})$ 


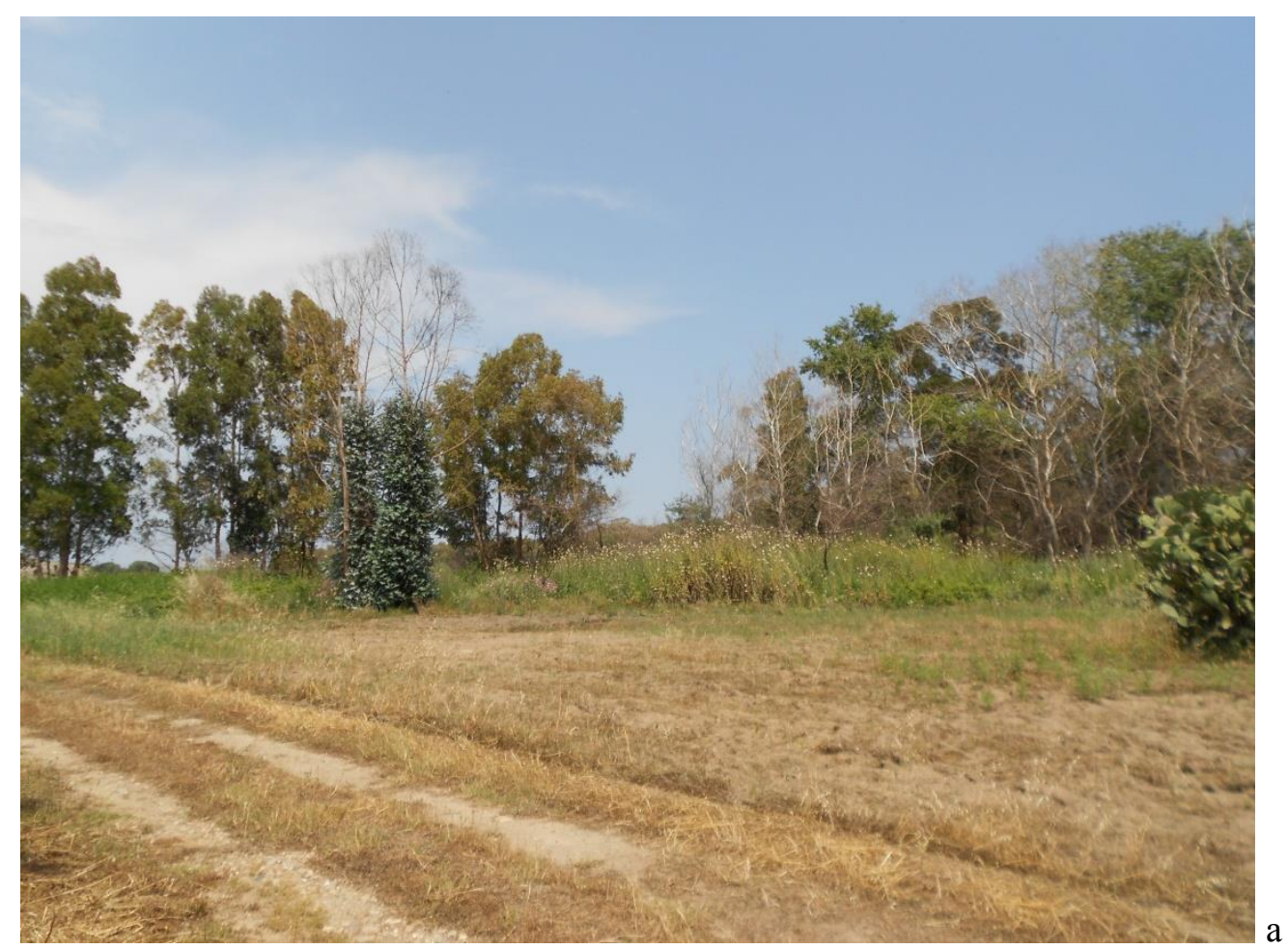

Il sito è interessato da coltivi che si estendono a ridosso delle aree a maggior valenza naturalistica $(\mathrm{a}, \mathrm{b})$

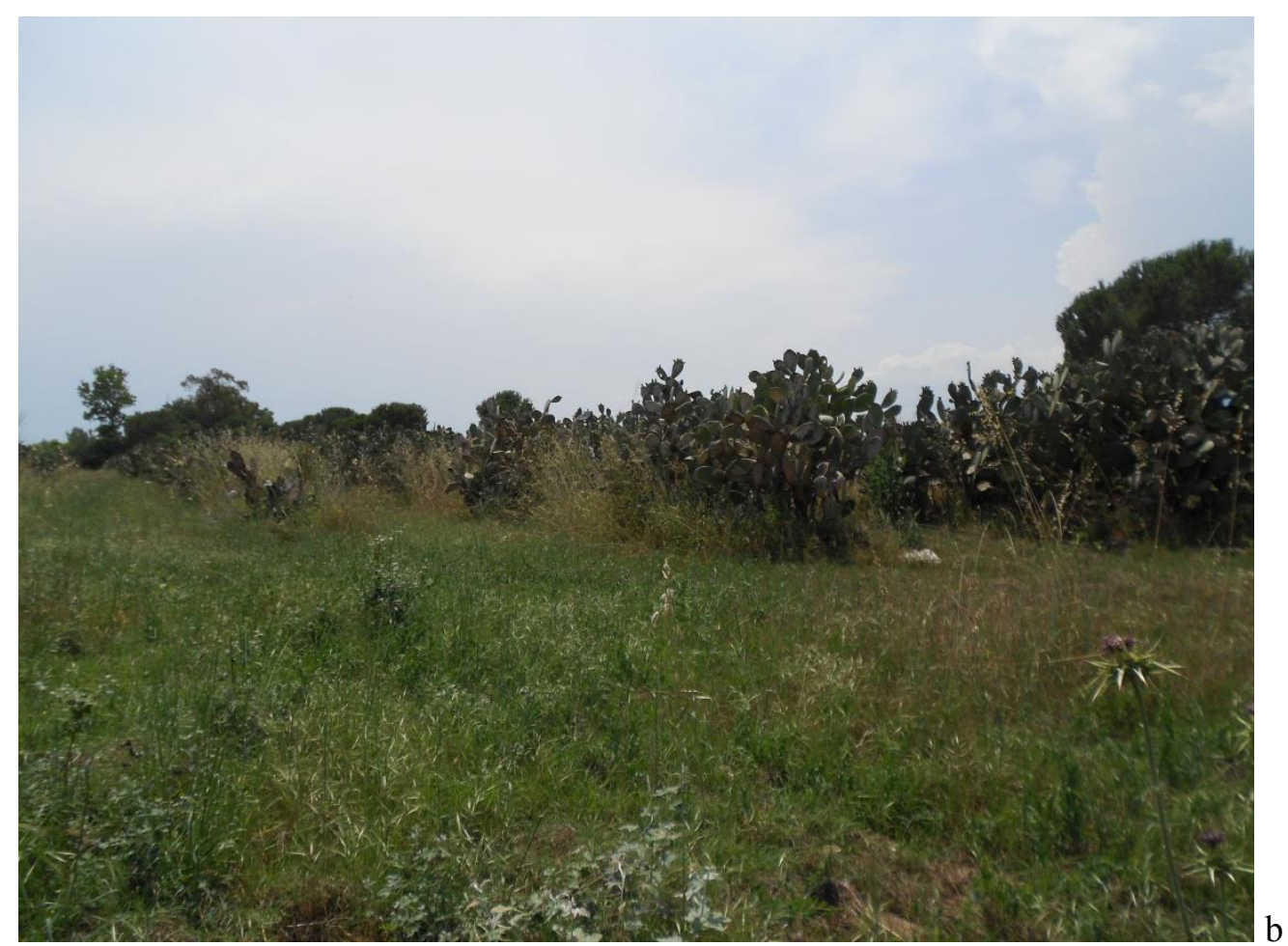


Arcidiacono C., et al.: Naturalità e Vulnerabilità Ambientale nei Siti Natura 2000 che

insistono sui corridoi ecologici del versante jonico calabrese. Caso studio SIC Foce Neto.

FP 129

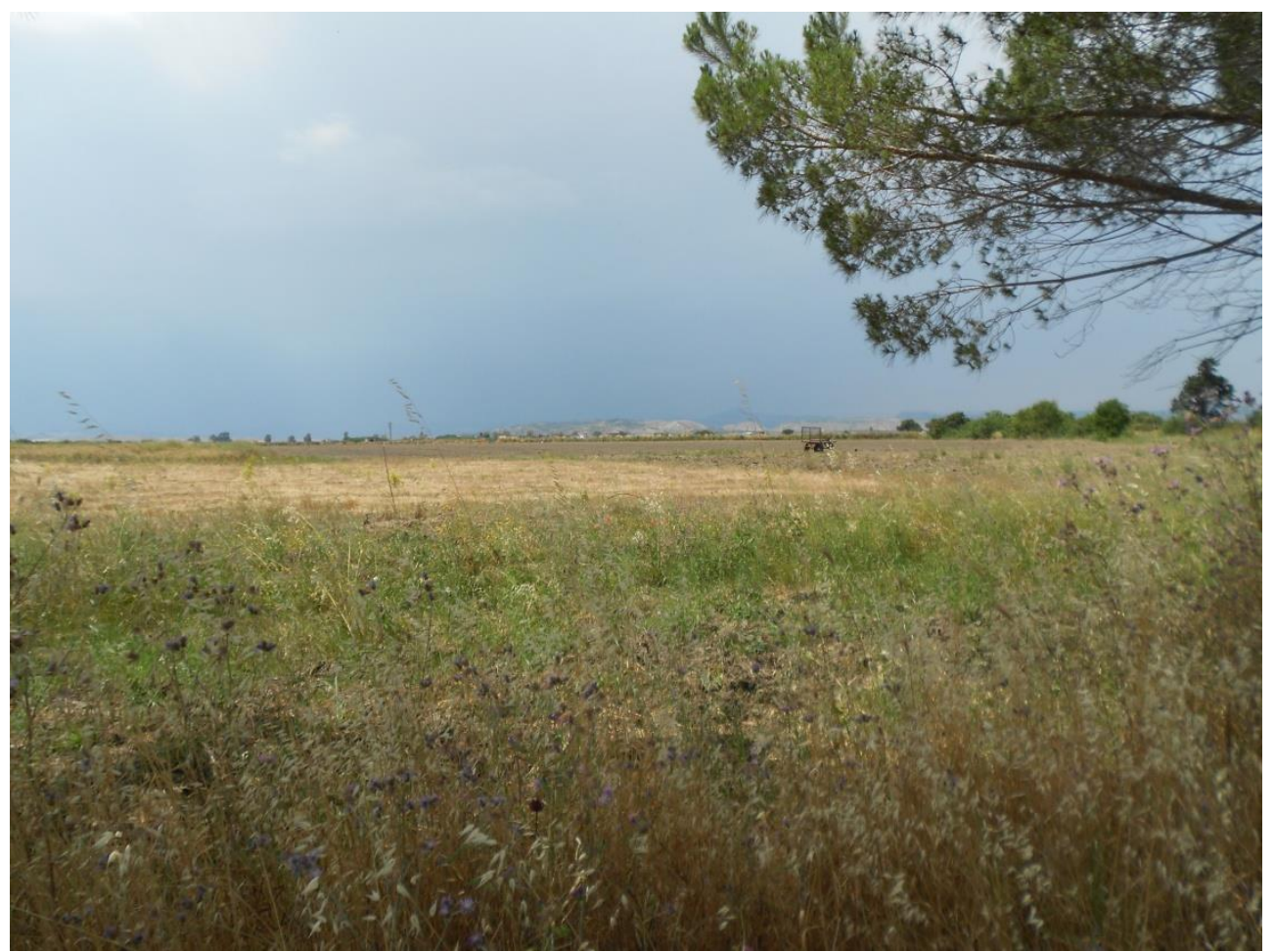

Aree coltivate con aspetti di vegetazione di tipo sinantropico

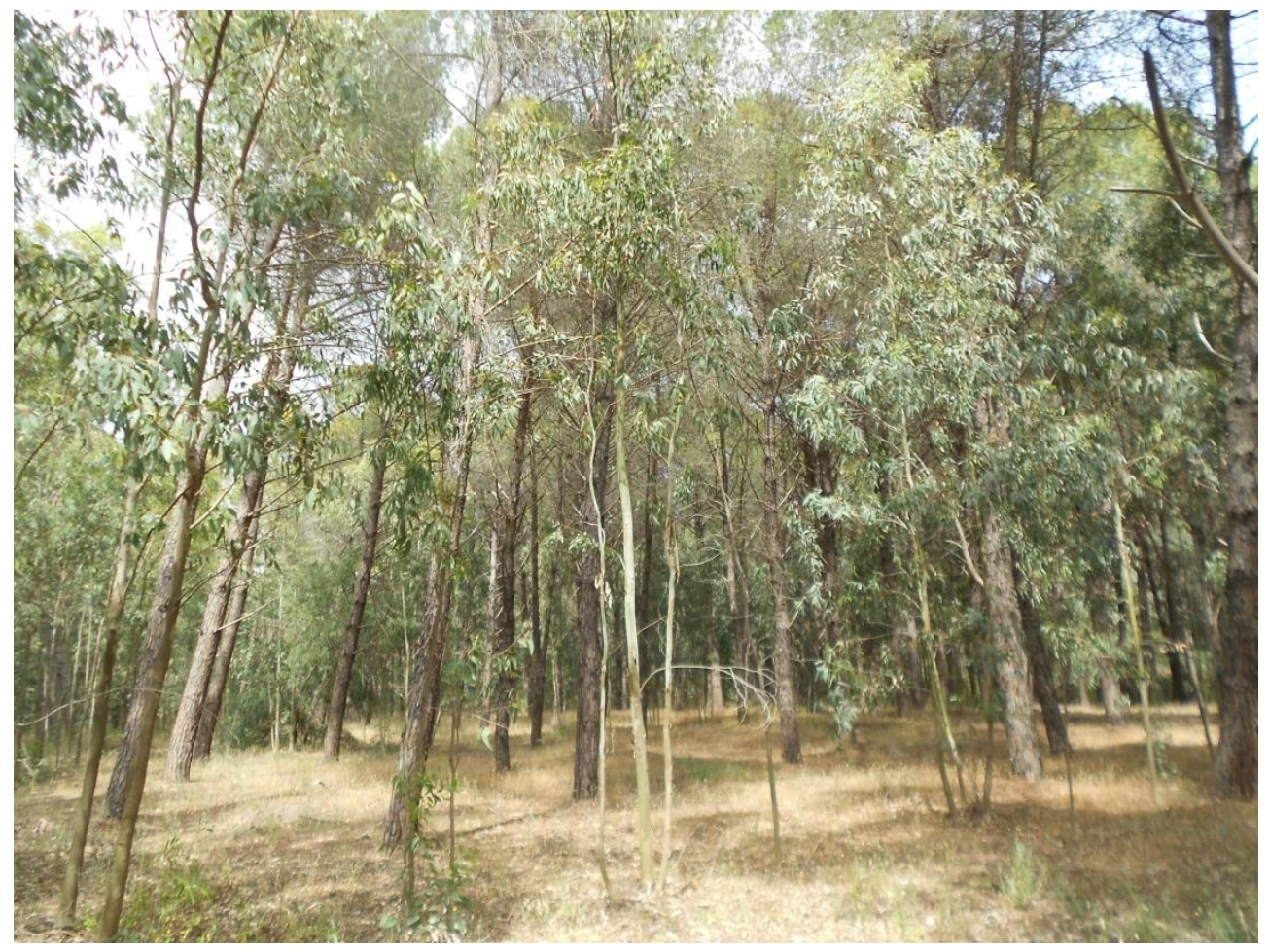

Rimboschimento con specie di Pinus sp pl. 
Arcidiacono C., et al.: Naturalità e Vulnerabilità Ambientale nei Siti Natura 2000 che

insistono sui corridoi ecologici del versante jonico calabrese. Caso studio SIC Foce Neto.

FP 130

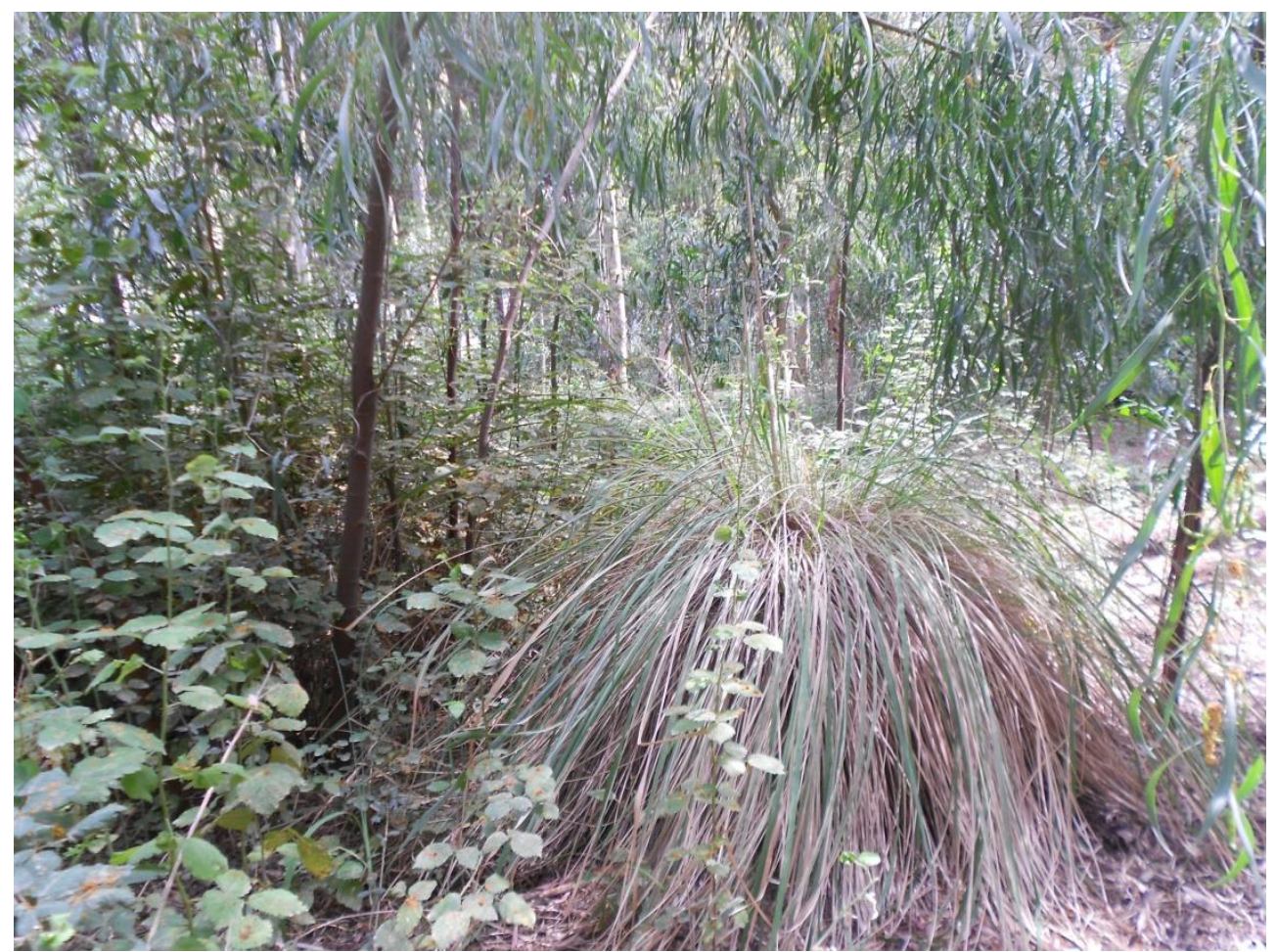

Nel rimboschimento a Pinus sono presenti differenti specie esotiche, come Cortaderia selloana in primo piano

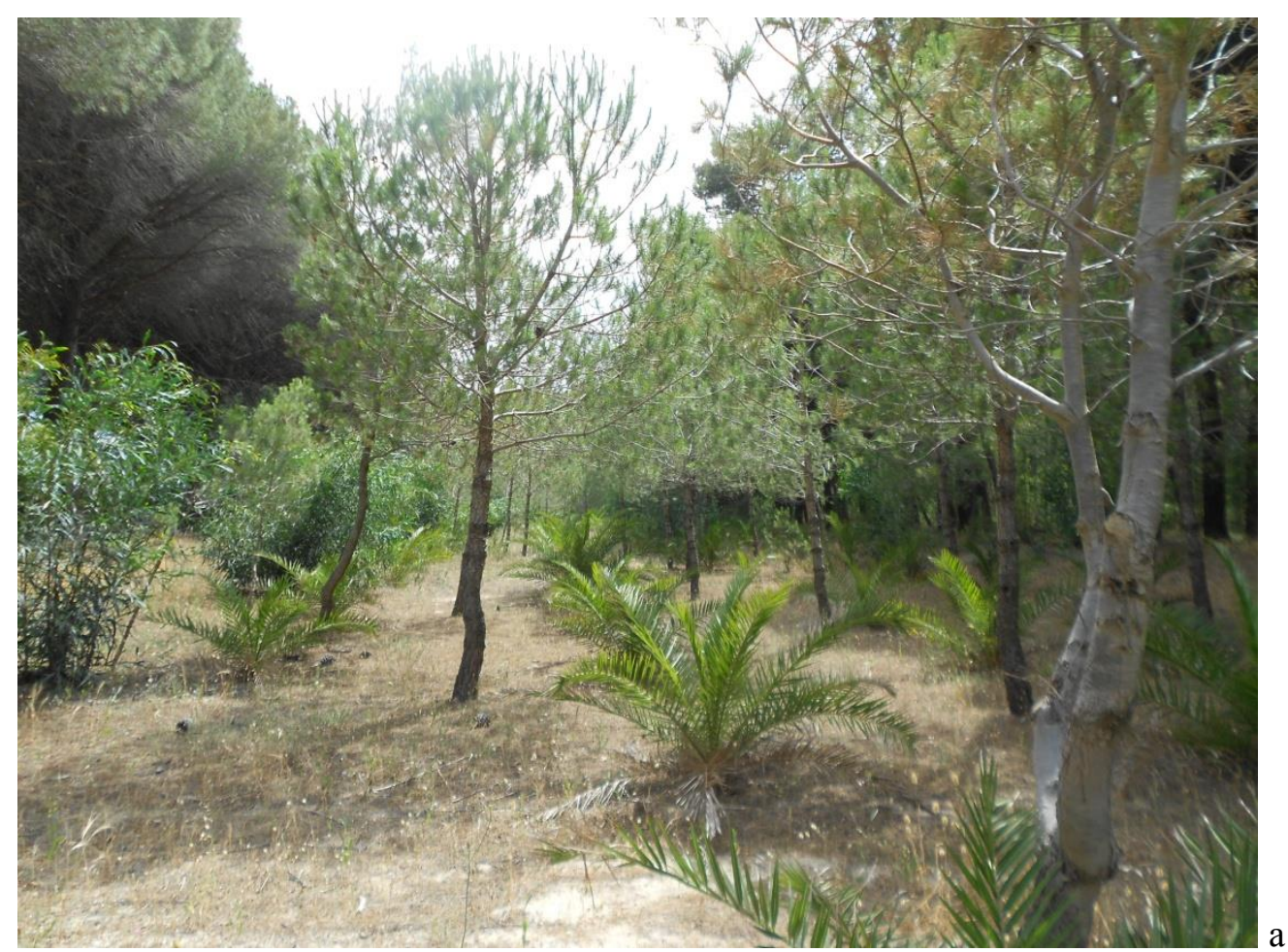

Rimboschimento di Pinus sp pl. con Phoenix canariensis (a, b, c, d) 
Arcidiacono C., et al.: Naturalità e Vulnerabilità Ambientale nei Siti Natura 2000 che

insistono sui corridoi ecologici del versante jonico calabrese. Caso studio SIC Foce Neto.

FP 131
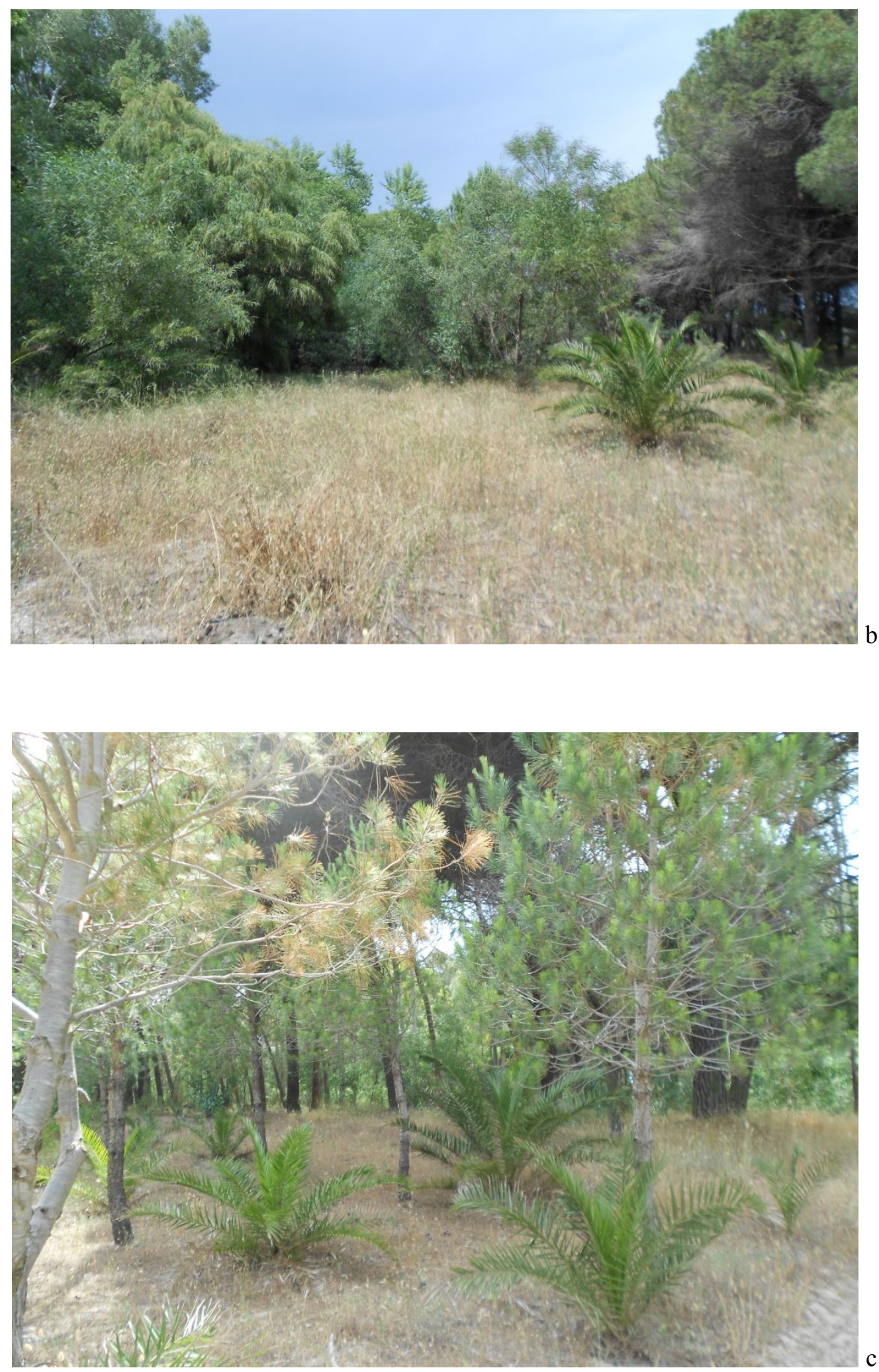
Arcidiacono C., et al.: Naturalità e Vulnerabilità Ambientale nei Siti Natura 2000 che

insistono sui corridoi ecologici del versante jonico calabrese. Caso studio SIC Foce Neto.

FP 132
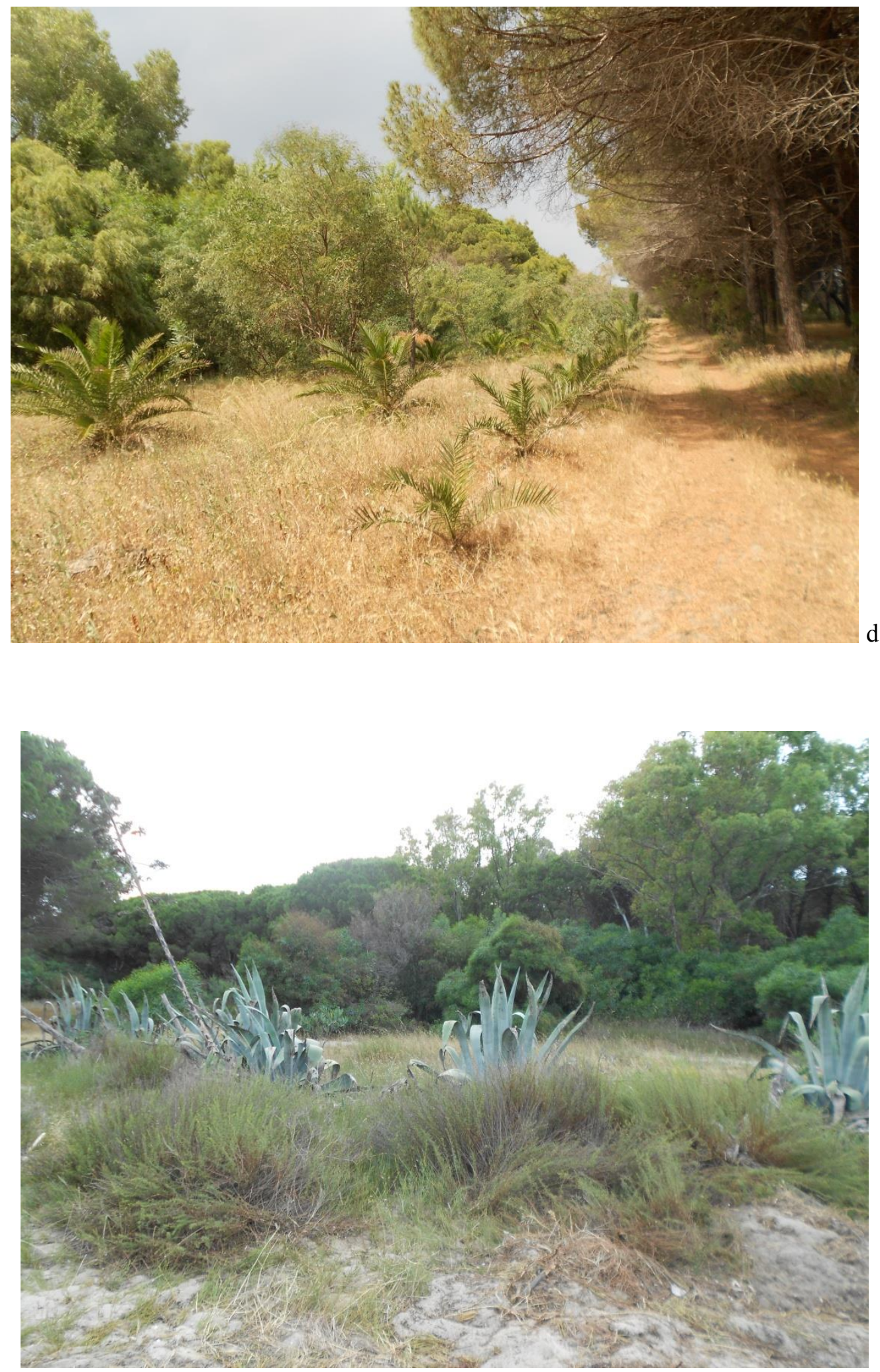

Agave americana sulla duna. Questa specie rappresenta una pressione e una minaccia per le cenosi naturali. 
Arcidiacono C., et al.: Naturalità e Vulnerabilità Ambientale nei Siti Natura 2000 che insistono sui corridoi ecologici del versante jonico calabrese. Caso studio SIC Foce Neto.

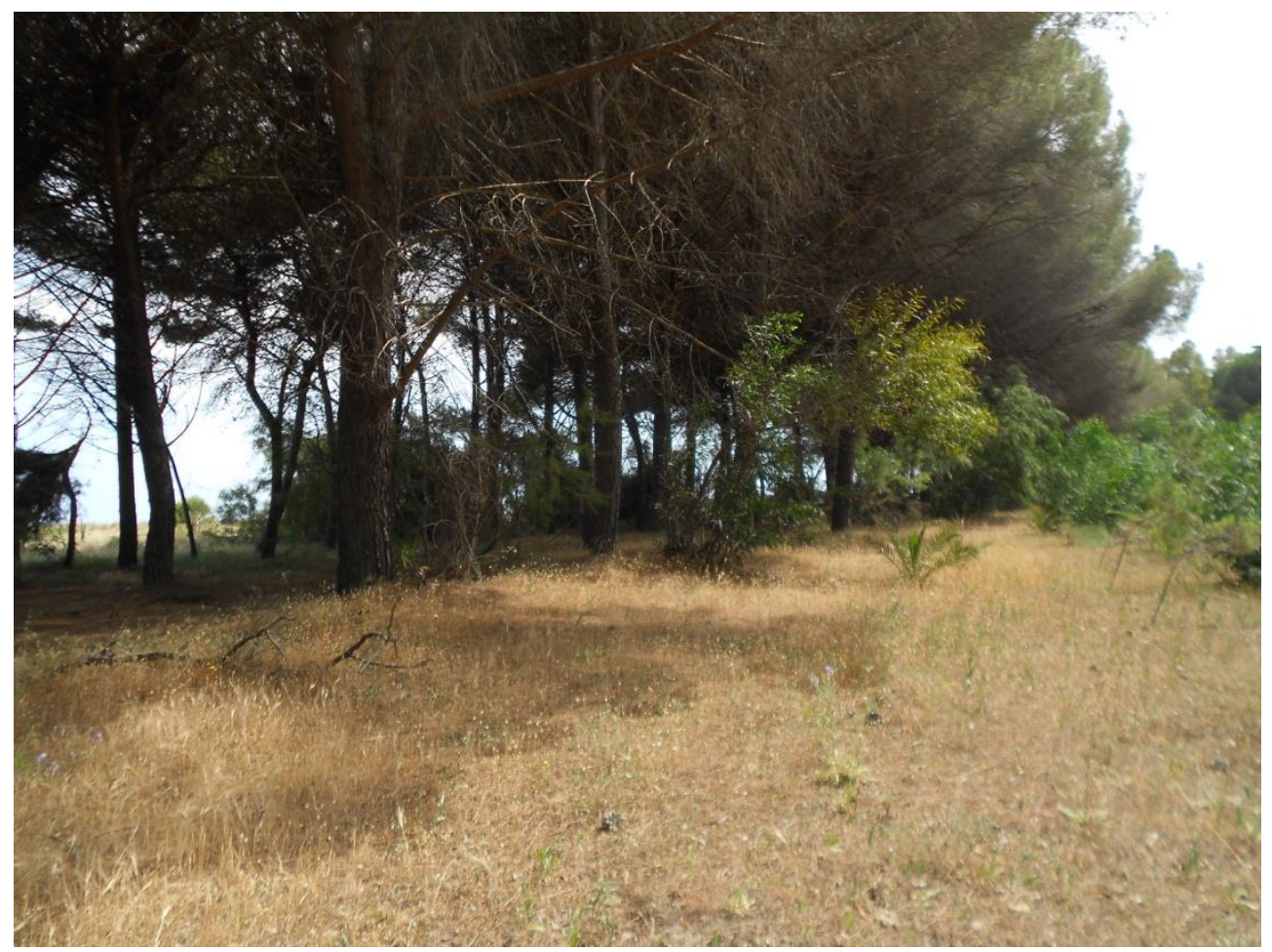

Di sotto il rimboschimento, il corteggio floristico non presenta gli aspetti della macchia, una rada vegetazione erbacea annuale di tipo ruderale

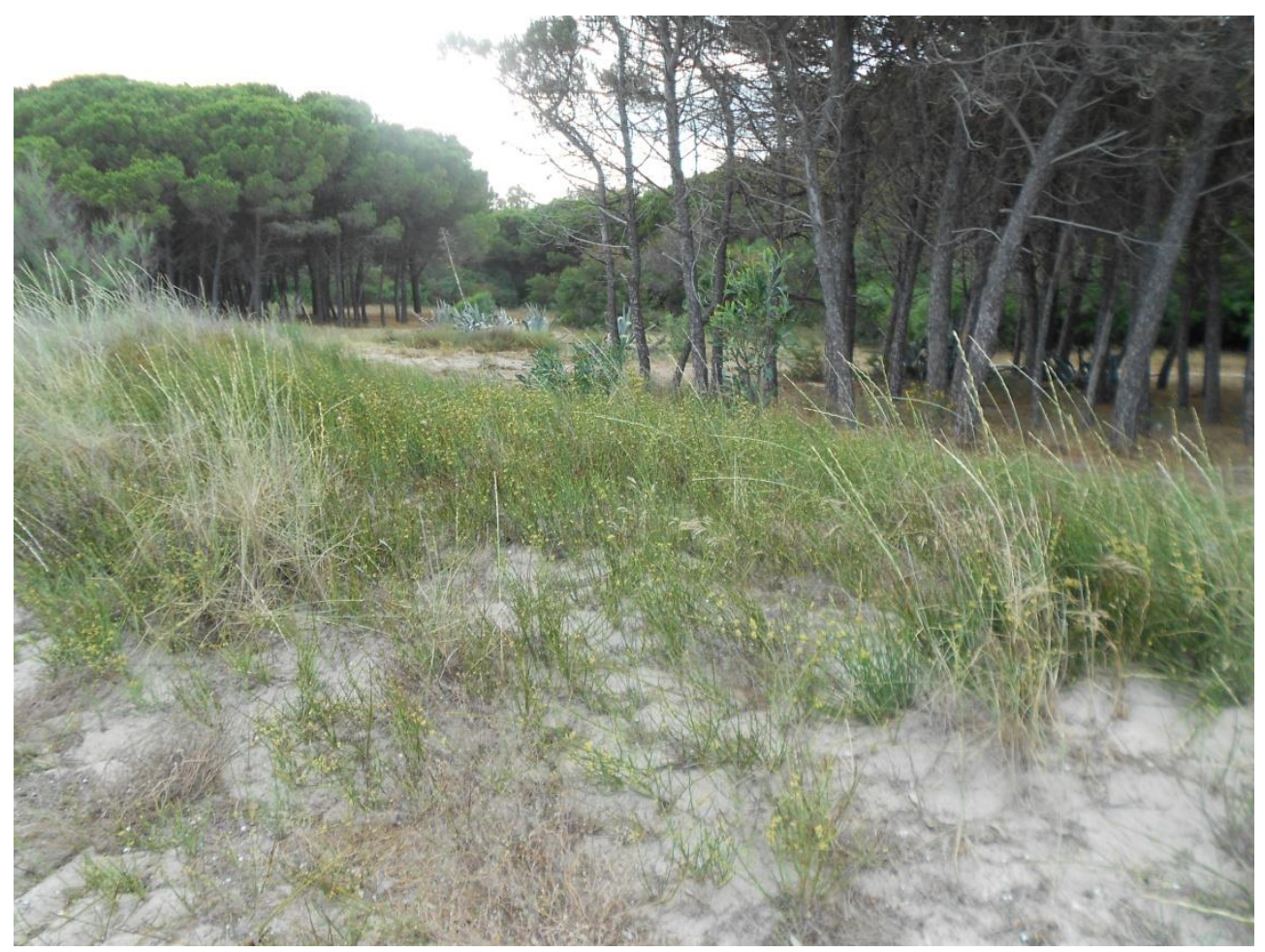

Aggruppamento ad Ephedra distachia, minacciata dalla rinnovazione della pineta 
Arcidiacono C., et al.: Naturalità e Vulnerabilità Ambientale nei Siti Natura 2000 che insistono sui corridoi ecologici del versante jonico calabrese. Caso studio SIC Foce Neto.

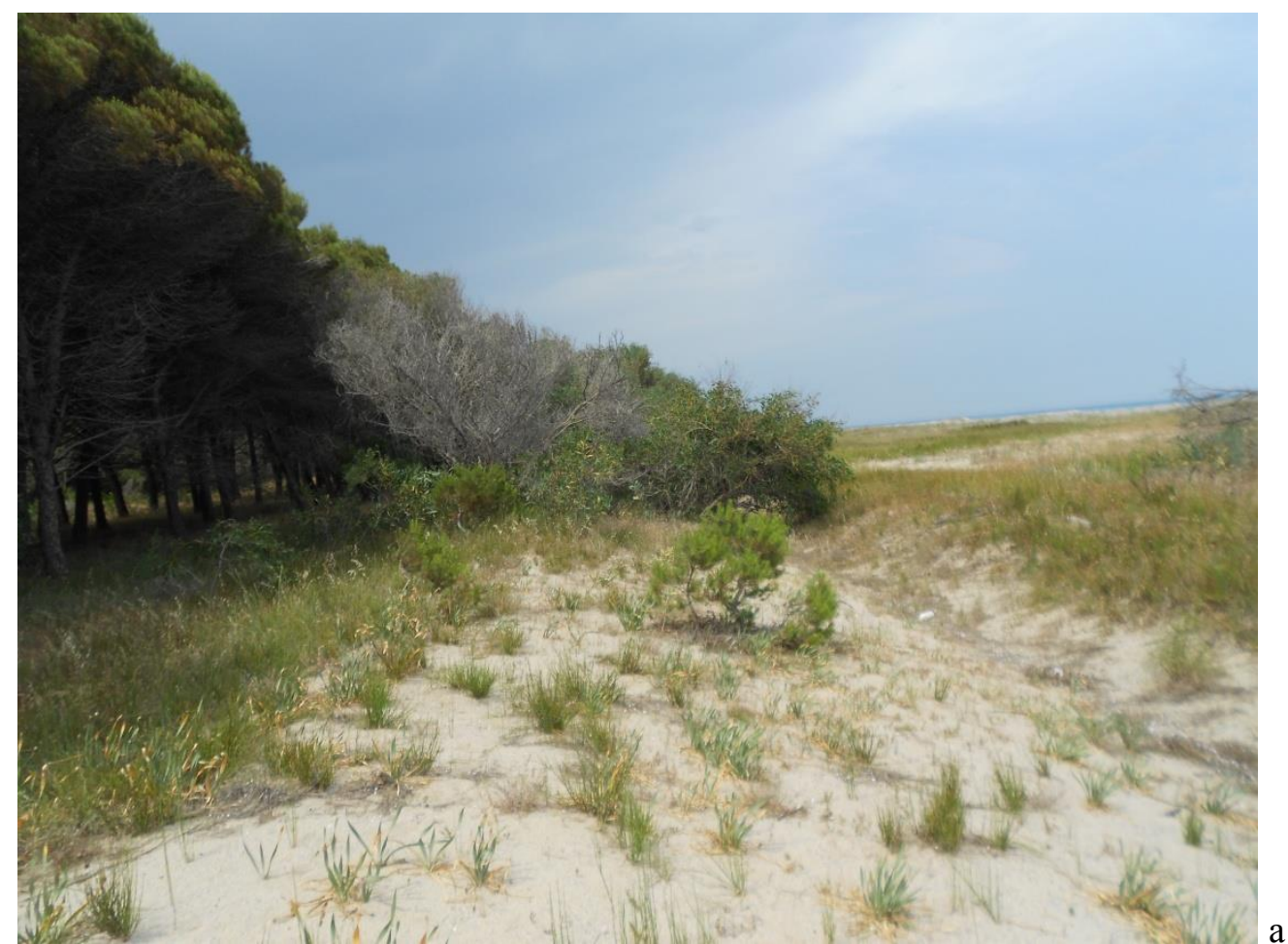

La rinnovazione spontanea del Pino e dell'Eucalipto a scapito delle cenosi e degli elementi floristici tipici degli ambienti di retroduna $(a, b)$

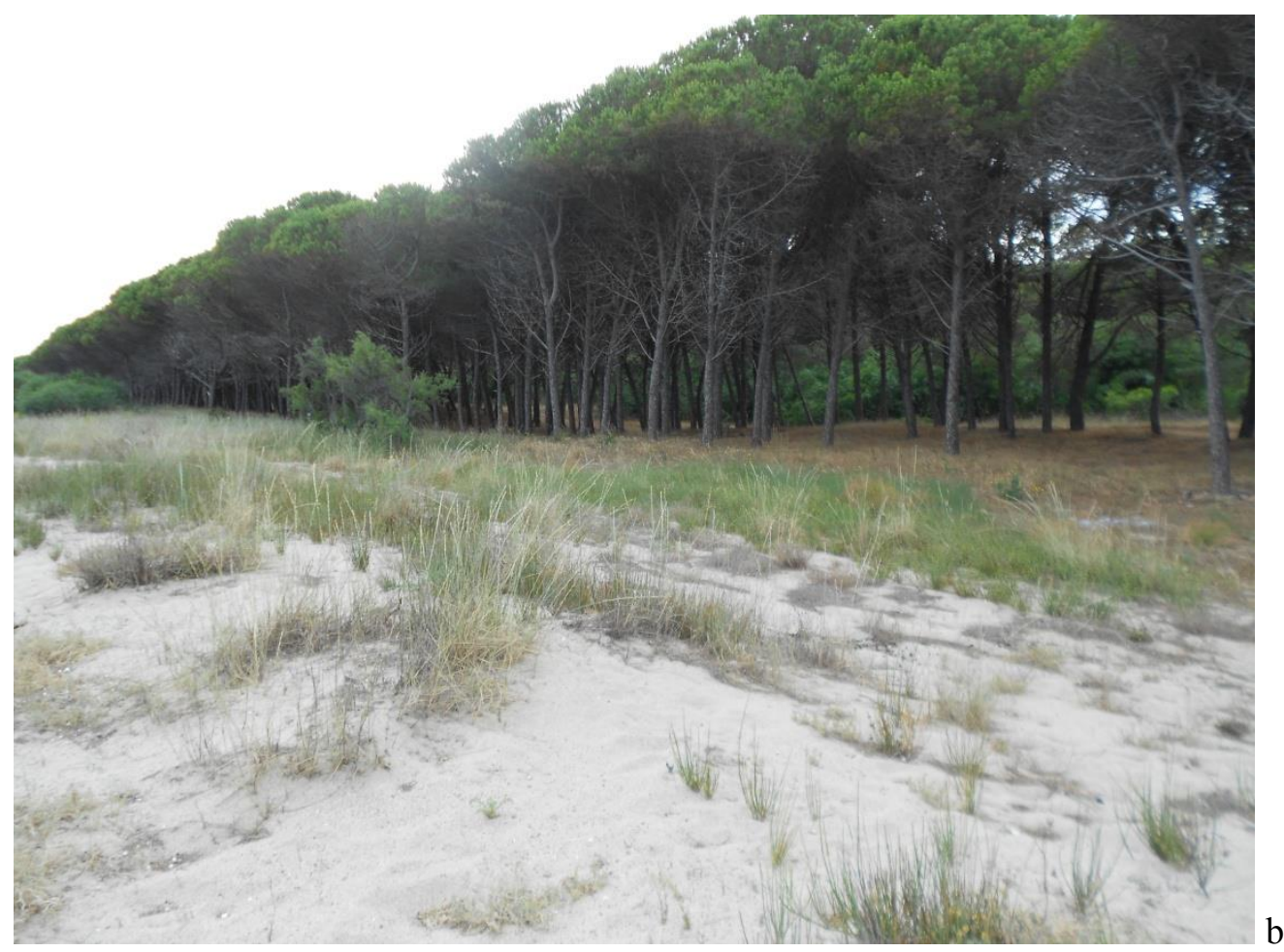


Arcidiacono C., et al.: Naturalità e Vulnerabilità Ambientale nei Siti Natura 2000 che

insistono sui corridoi ecologici del versante jonico calabrese. Caso studio SIC Foce Neto.

FP 135

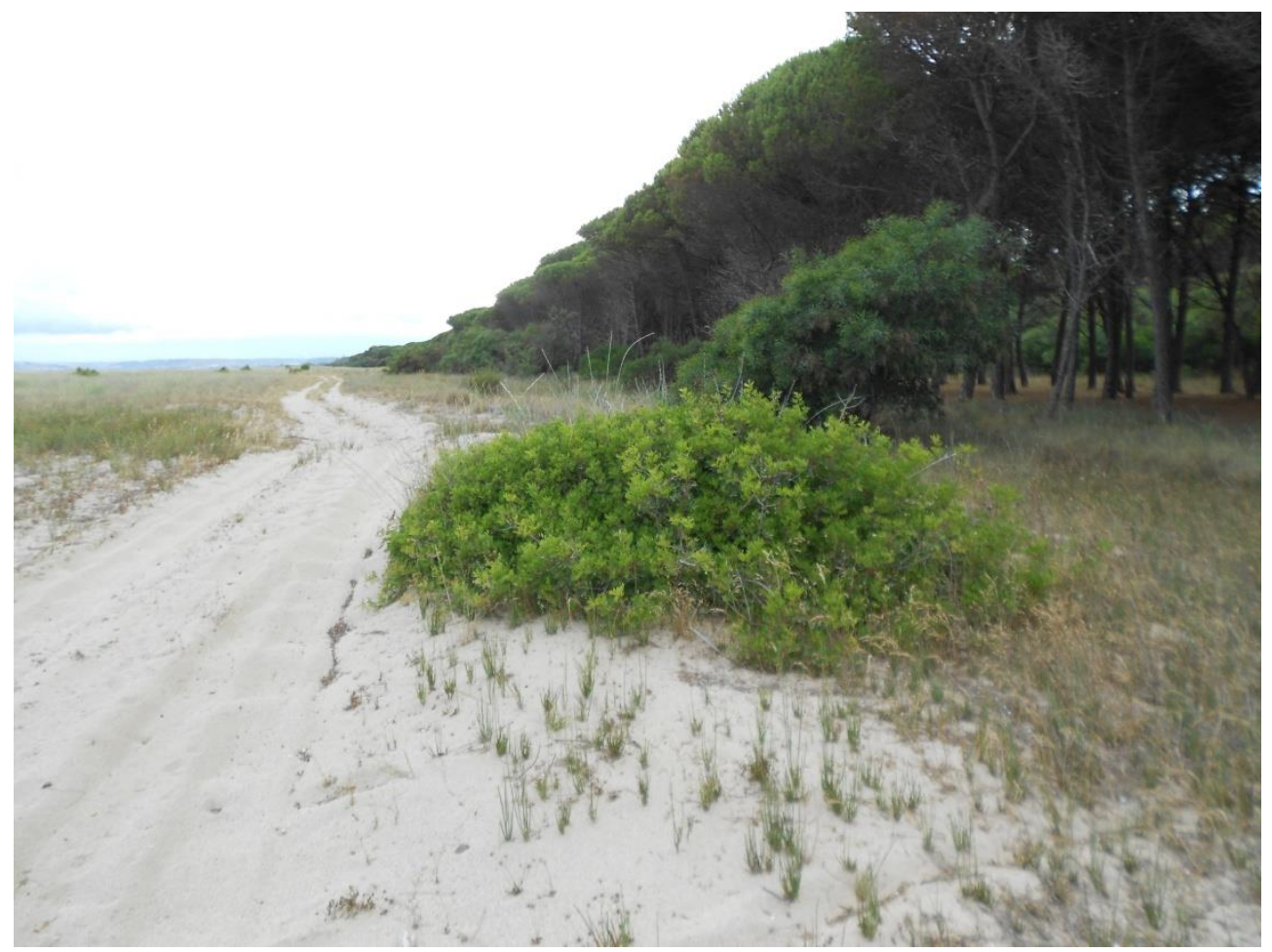

Pistacia lentiscus in primo piano, un elemento residuale della macchia

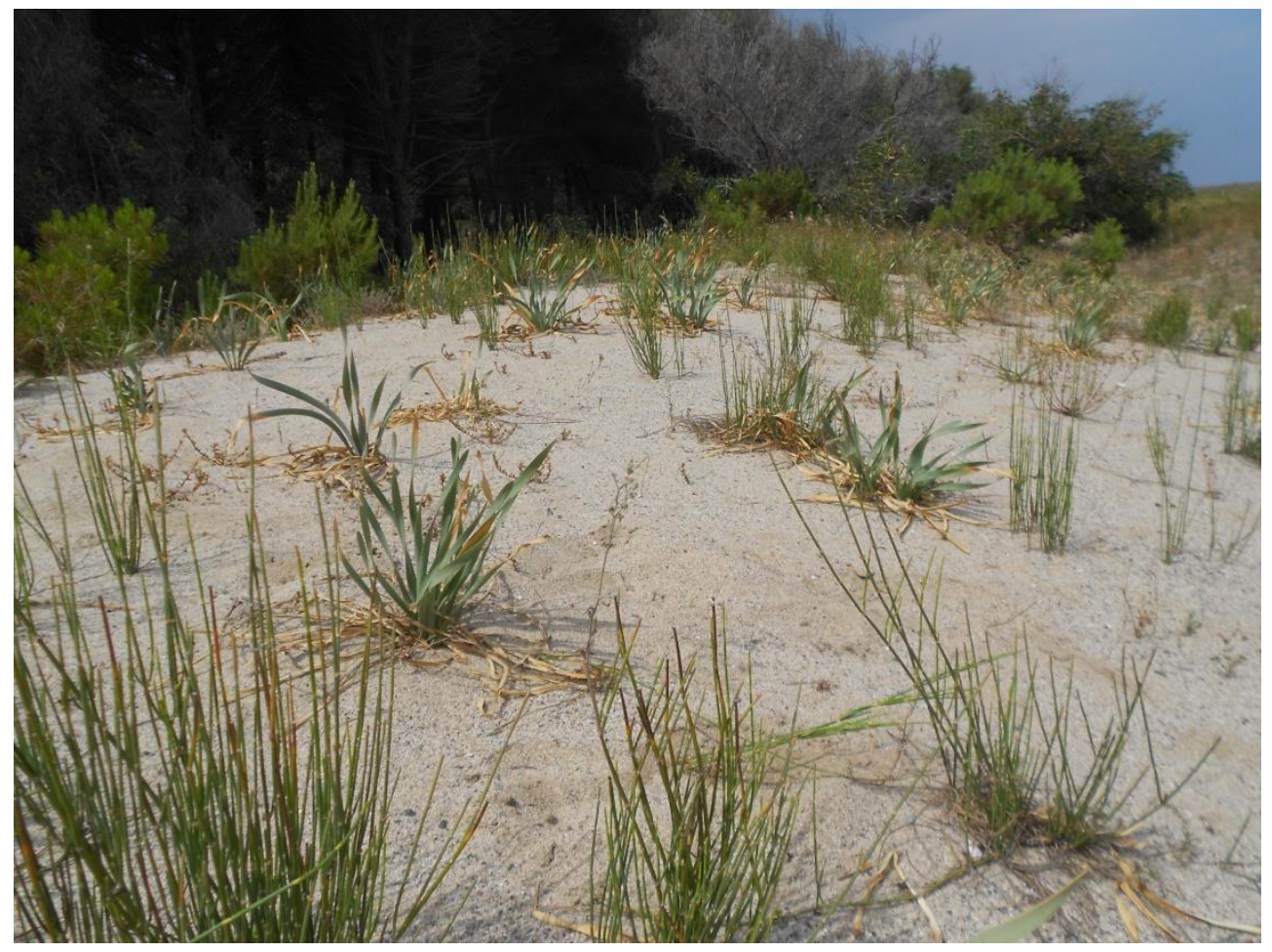

Pancratium maritimum ed Ephedra distachia sul cordone dunale 


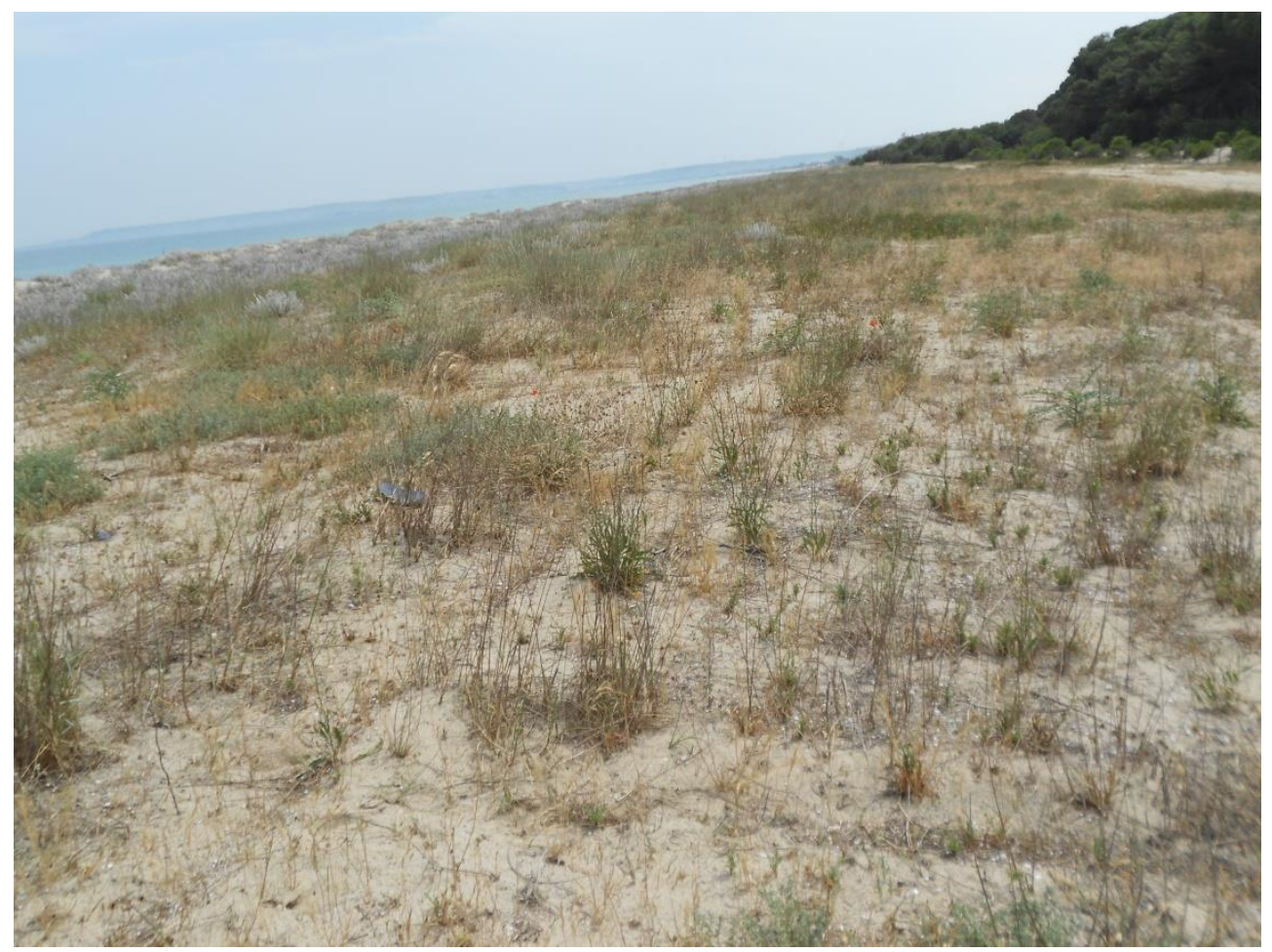

Il Sileno nicaeensis-Ononidetum variegatae sulle dune embrionali forma un mosaico con la vegetazione perenne dell'Echinophoro spinosae-Elytrigietum junceae.

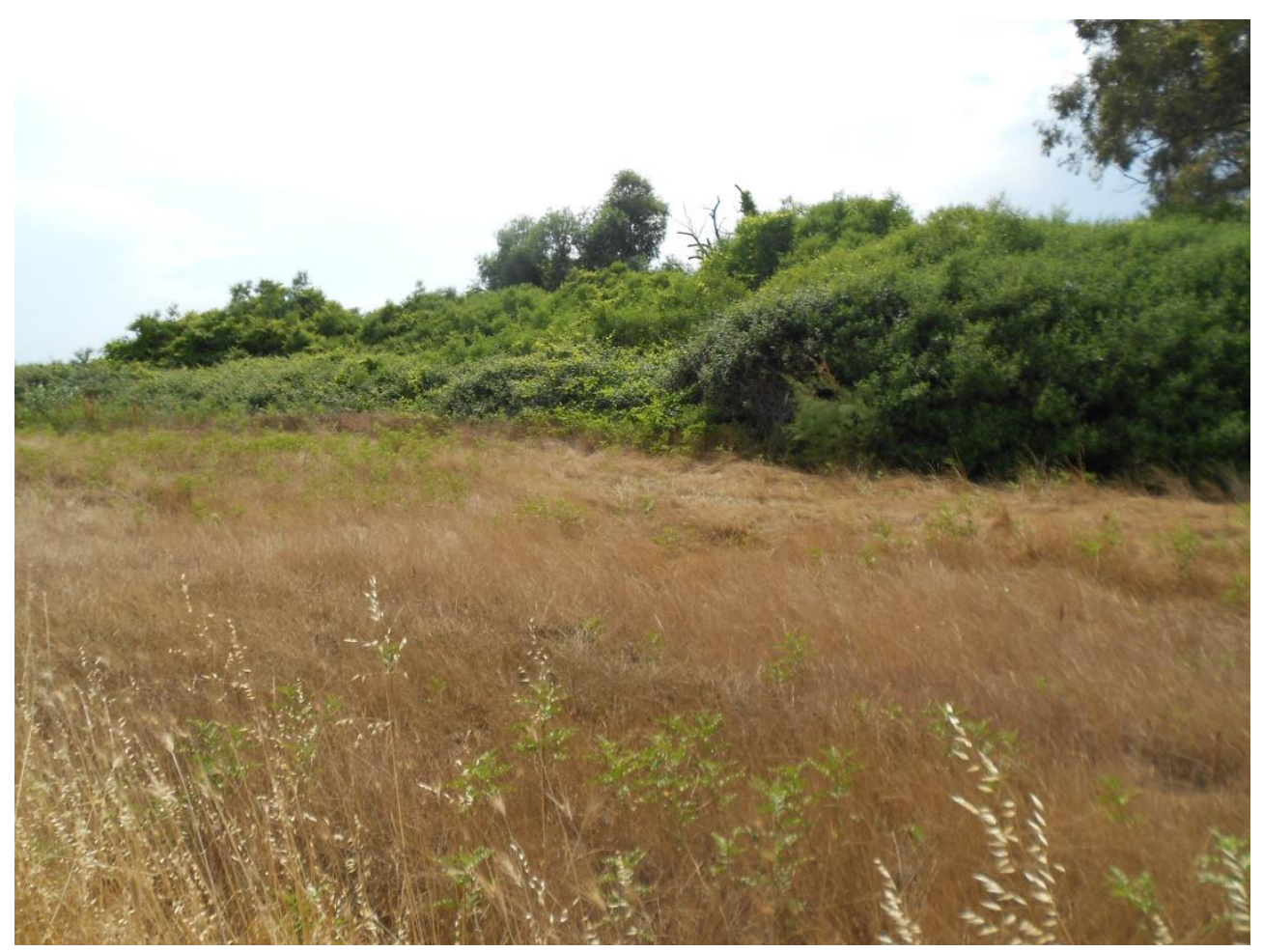

Frammentate dalle superfici agricole, sono presenti cenosi caratterizzate dalla dominanza di Vitex agnus-castus, cui si associano Tamarix africana, Prunus spinosa, Rubus ulmifolius, Rosa sempervirens, Vitis sylvestris, ecc. 
Arcidiacono C., et al.: Naturalità e Vulnerabilità Ambientale nei Siti Natura 2000 che

insistono sui corridoi ecologici del versante jonico calabrese. Caso studio SIC Foce Neto.

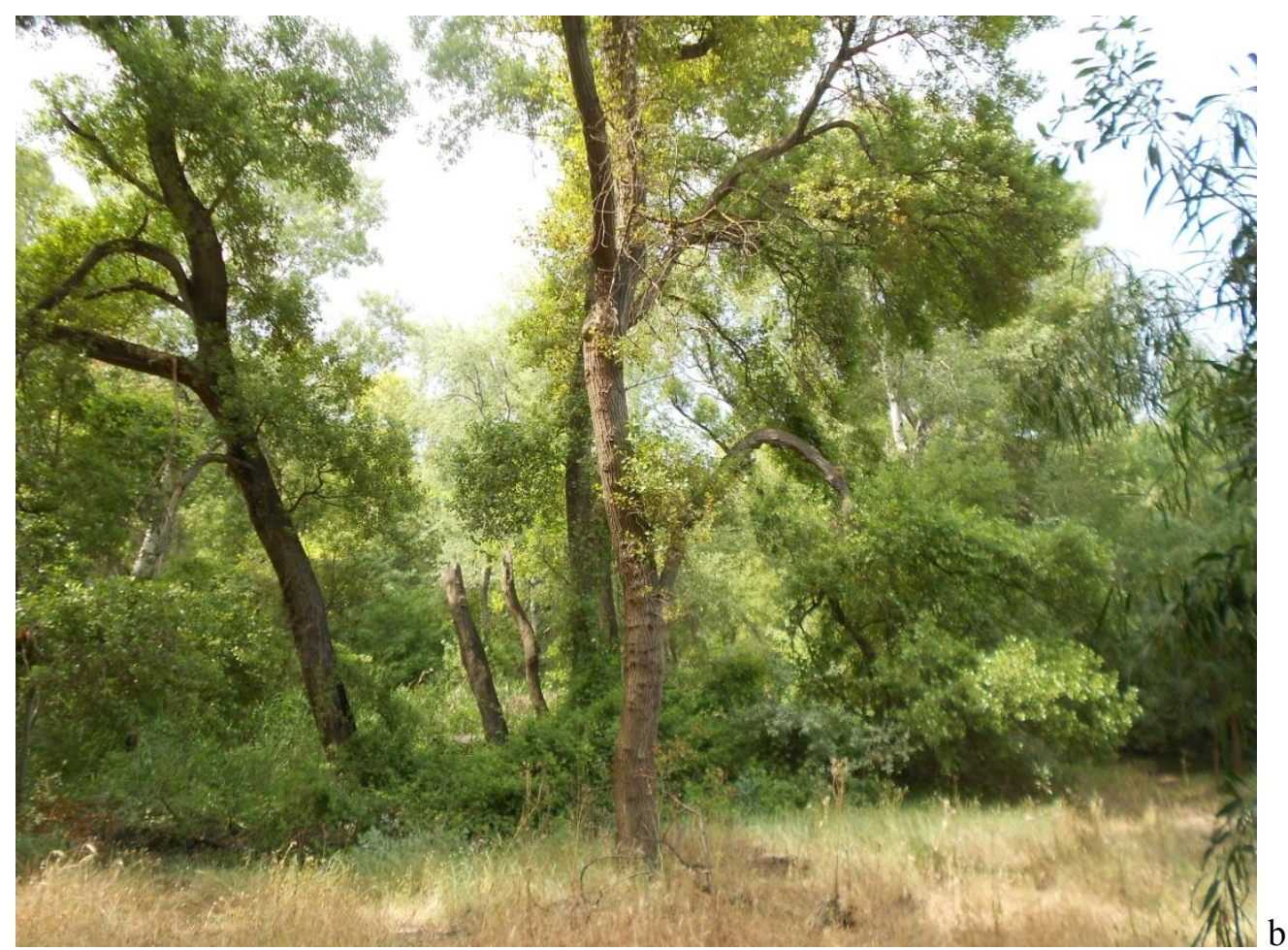

Lembi residuali del bosco edafoigraofilo a Salix alba e Populus alba (a,b,c, d)

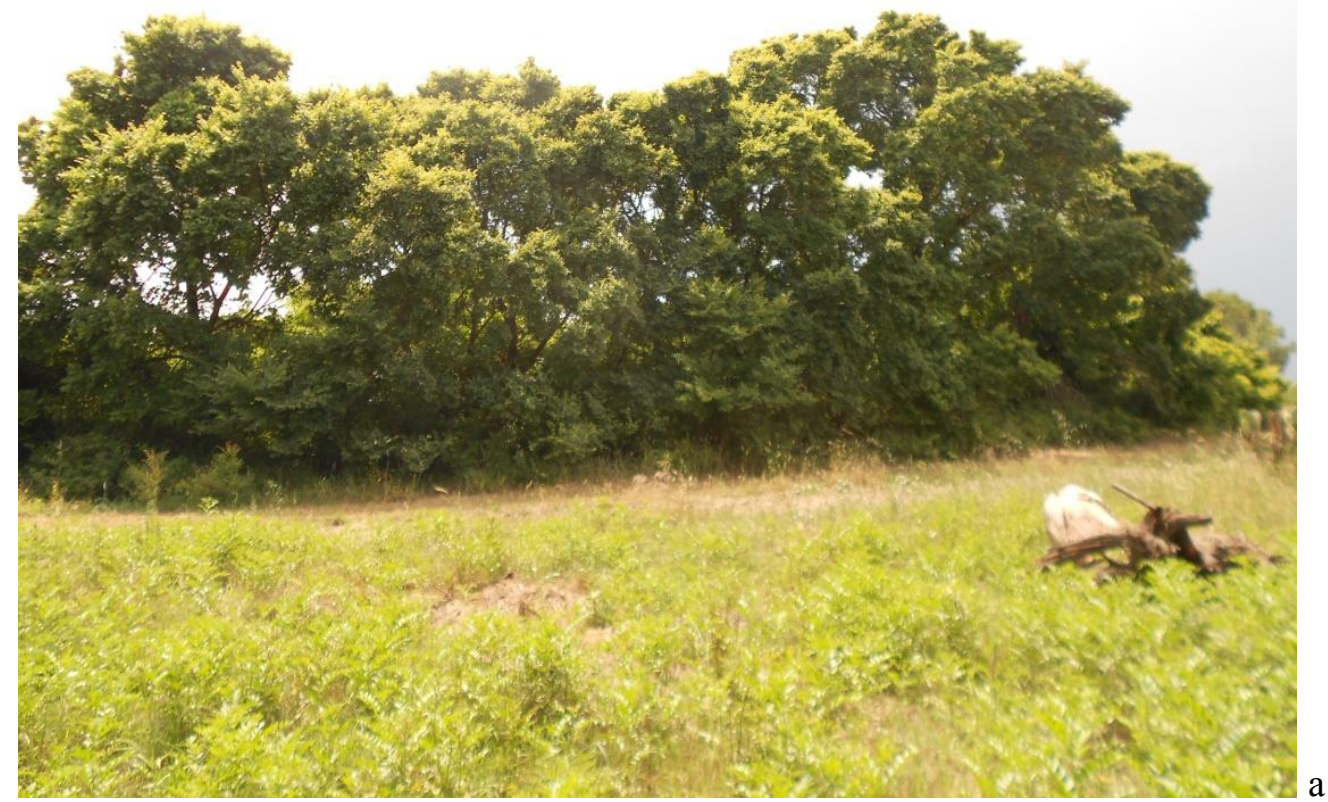


Arcidiacono C., et al.: Naturalità e Vulnerabilità Ambientale nei Siti Natura 2000 che

insistono sui corridoi ecologici del versante jonico calabrese. Caso studio SIC Foce Neto.

FP 138
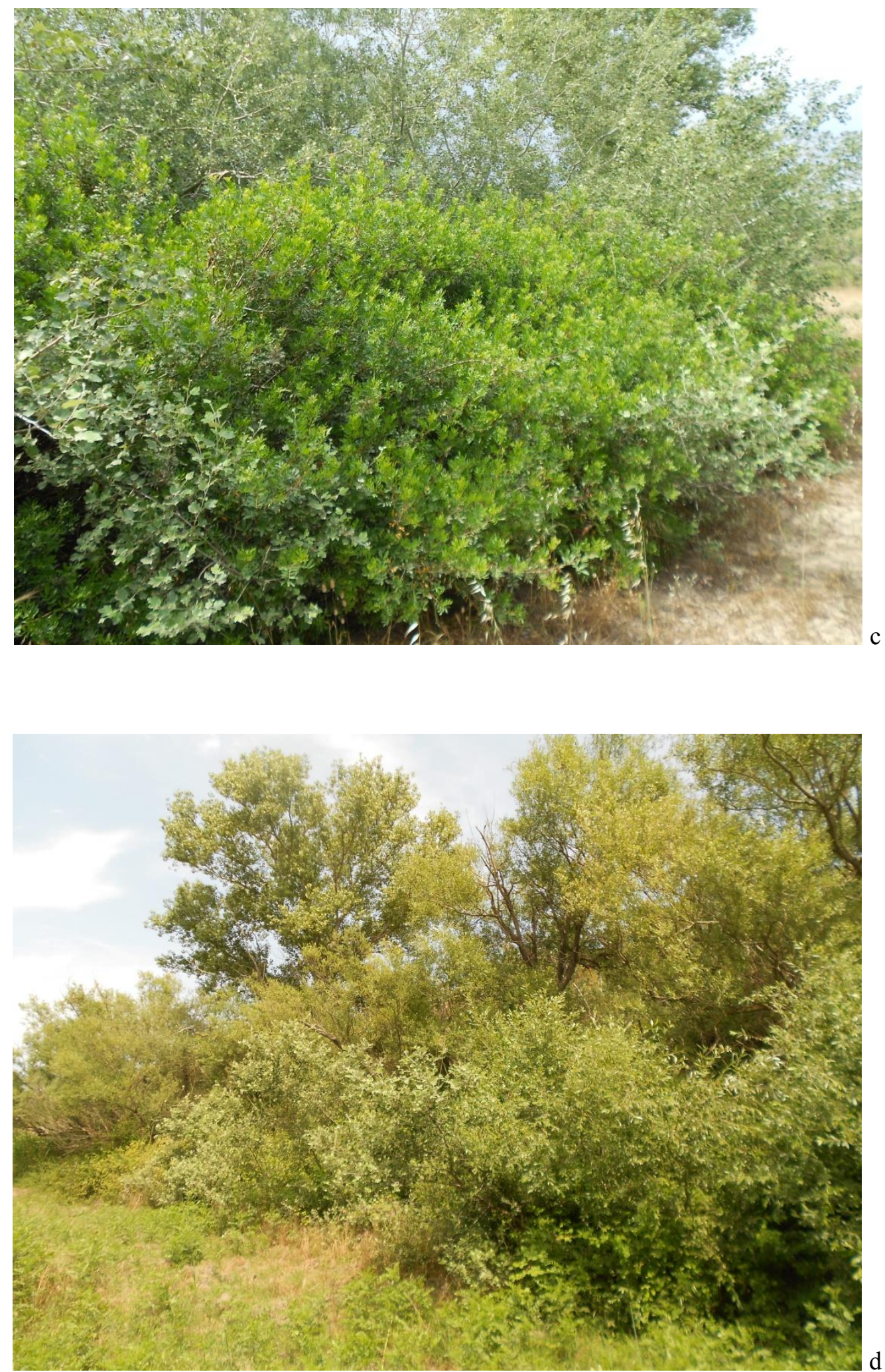
Arcidiacono C., et al.: Naturalità e Vulnerabilità Ambientale nei Siti Natura 2000 che

insistono sui corridoi ecologici del versante jonico calabrese. Caso studio SIC Foce Neto.

FP 139

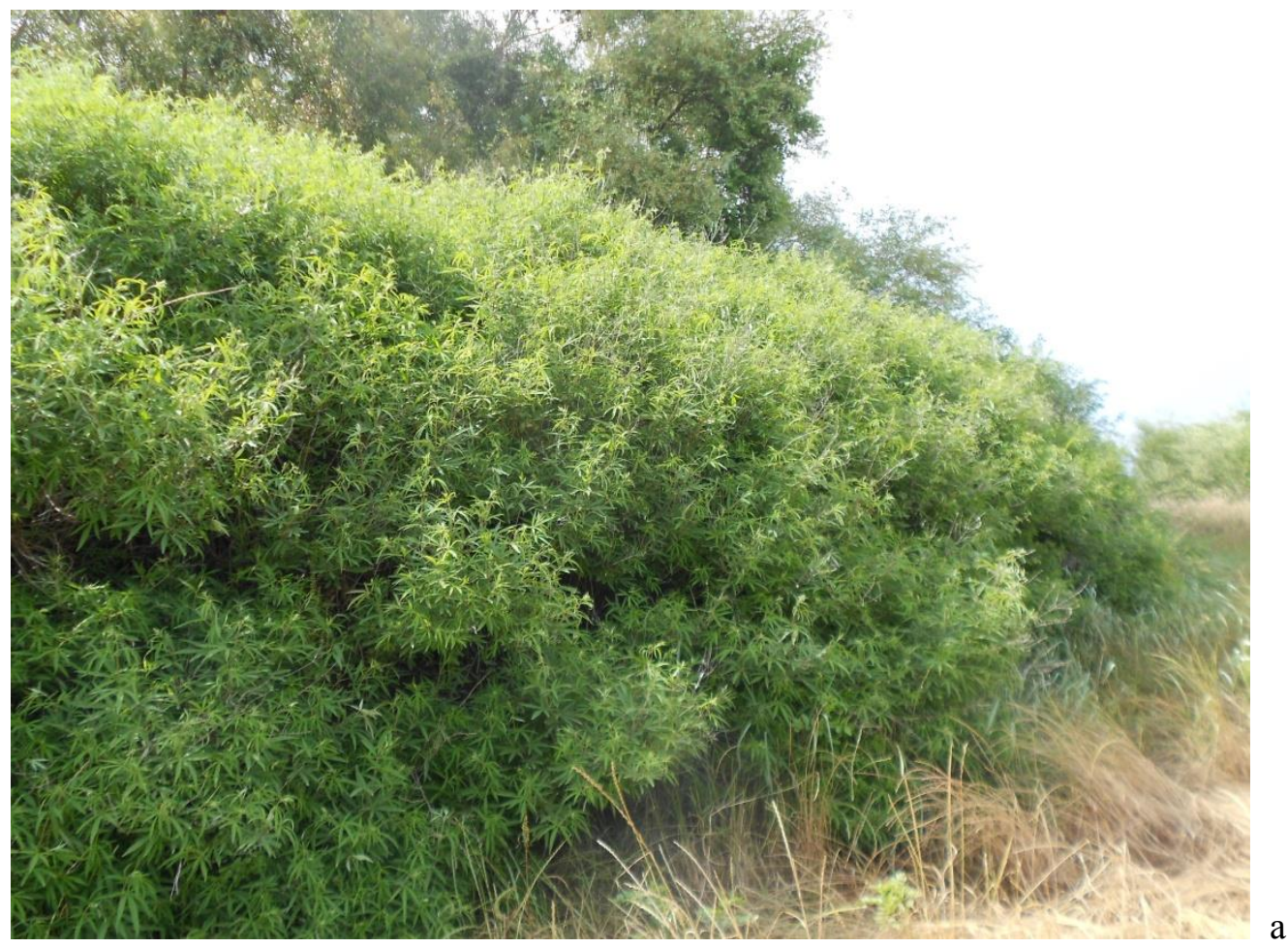

Aspetti del Tamarici africanae-Viticetum agni-casti Brullo \& Spampinato 1997 (a,b,c)

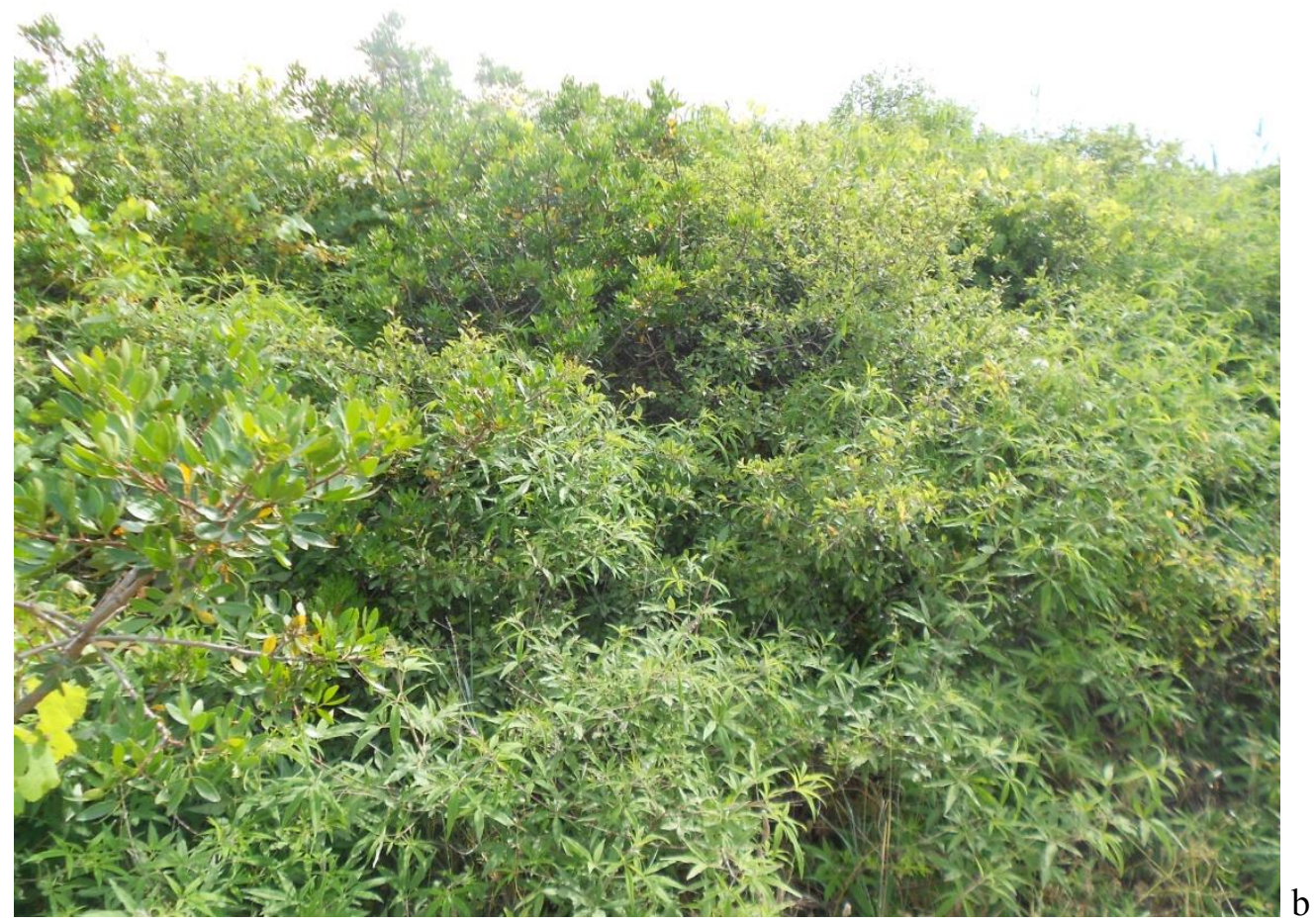


Arcidiacono C., et al.: Naturalità e Vulnerabilità Ambientale nei Siti Natura 2000 che insistono sui corridoi ecologici del versante jonico calabrese. Caso studio SIC Foce Neto.
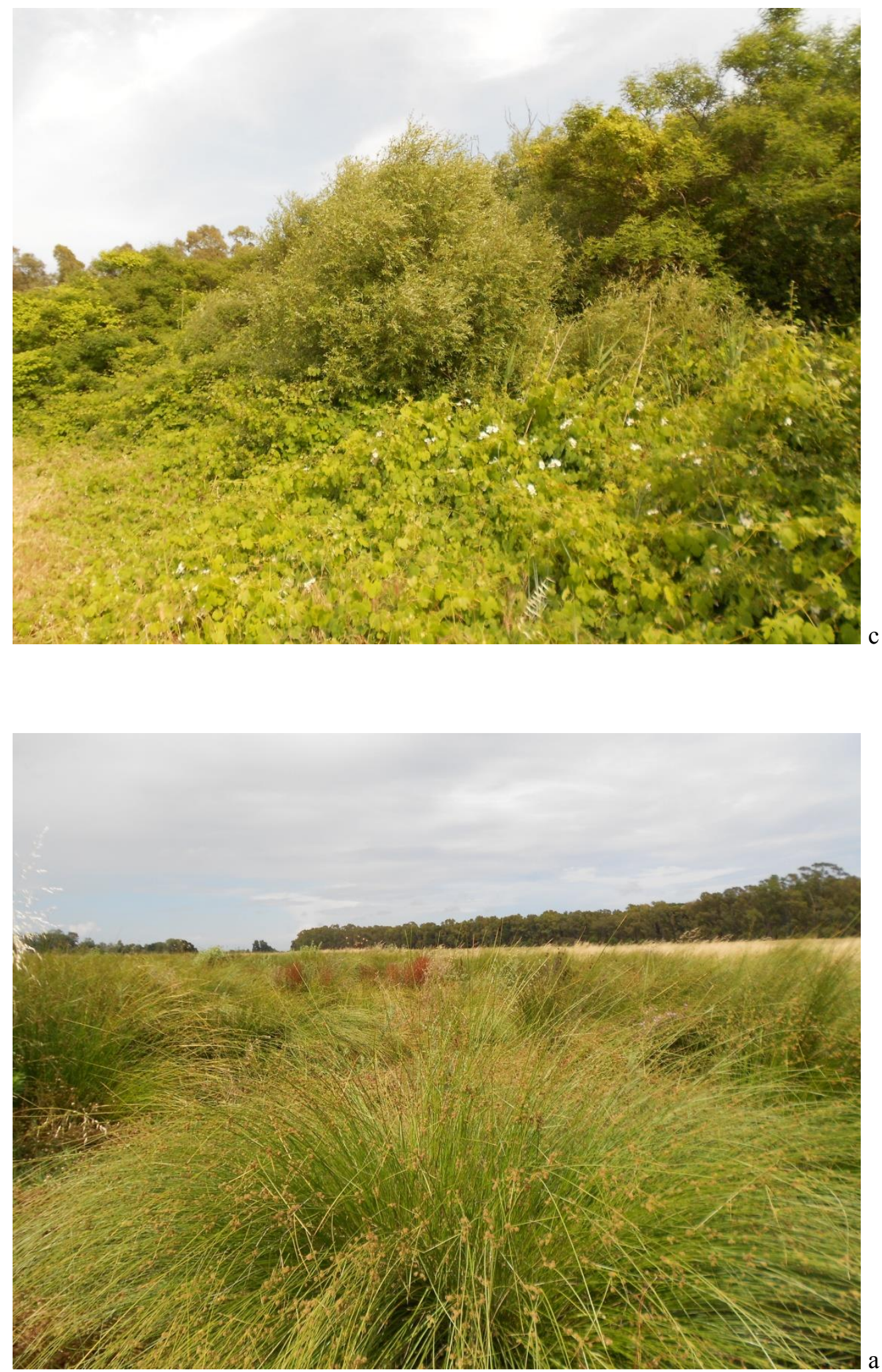

Pascoli inondati mediterranei (Juncetalia maritimi) $(\mathrm{a}, \mathrm{b})$ 
Arcidiacono C., et al.: Naturalità e Vulnerabilità Ambientale nei Siti Natura 2000 che insistono sui corridoi ecologici del versante jonico calabrese. Caso studio SIC Foce Neto.
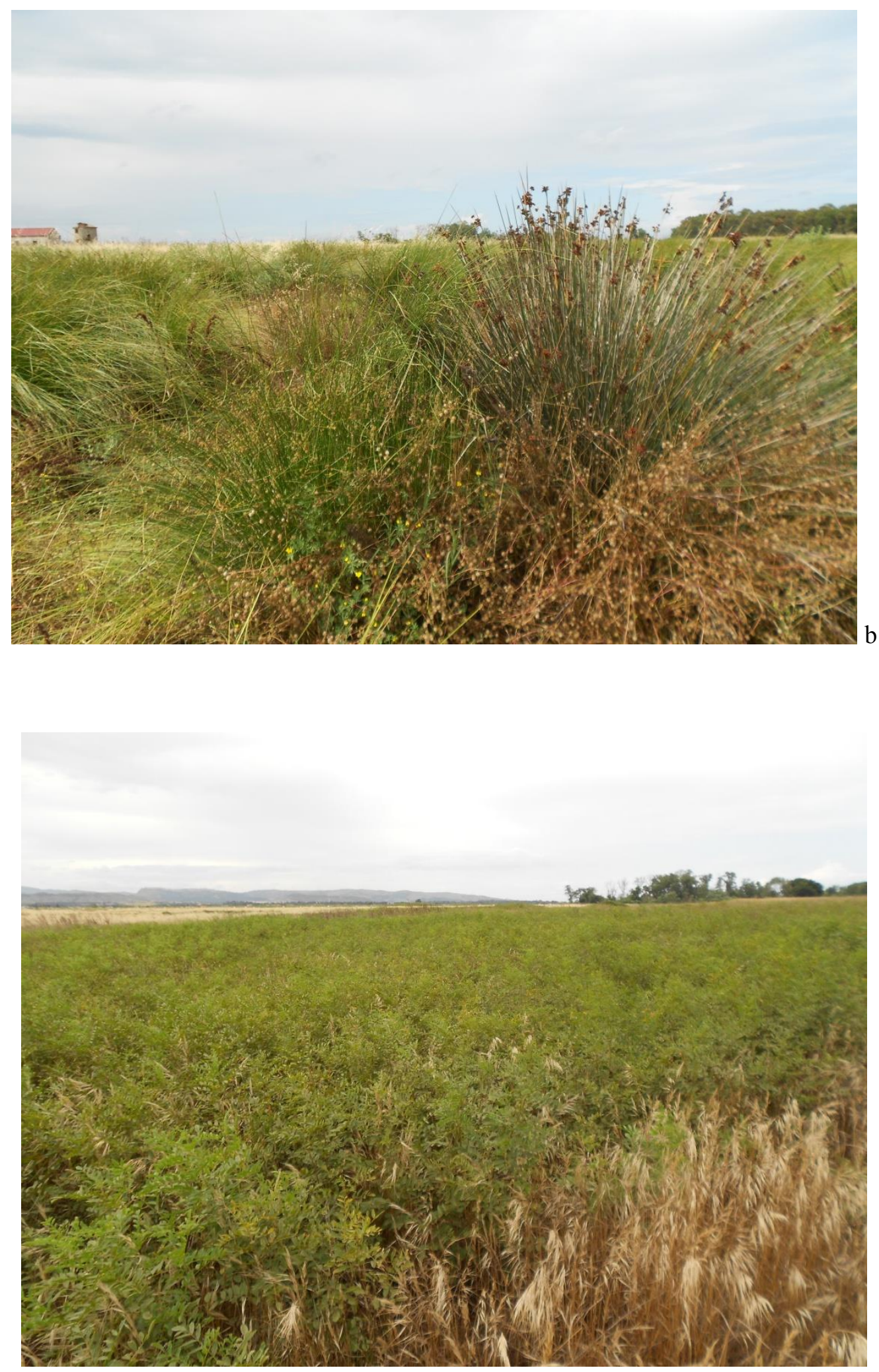

Aggruppamento a Glycyrrhiza glabra 


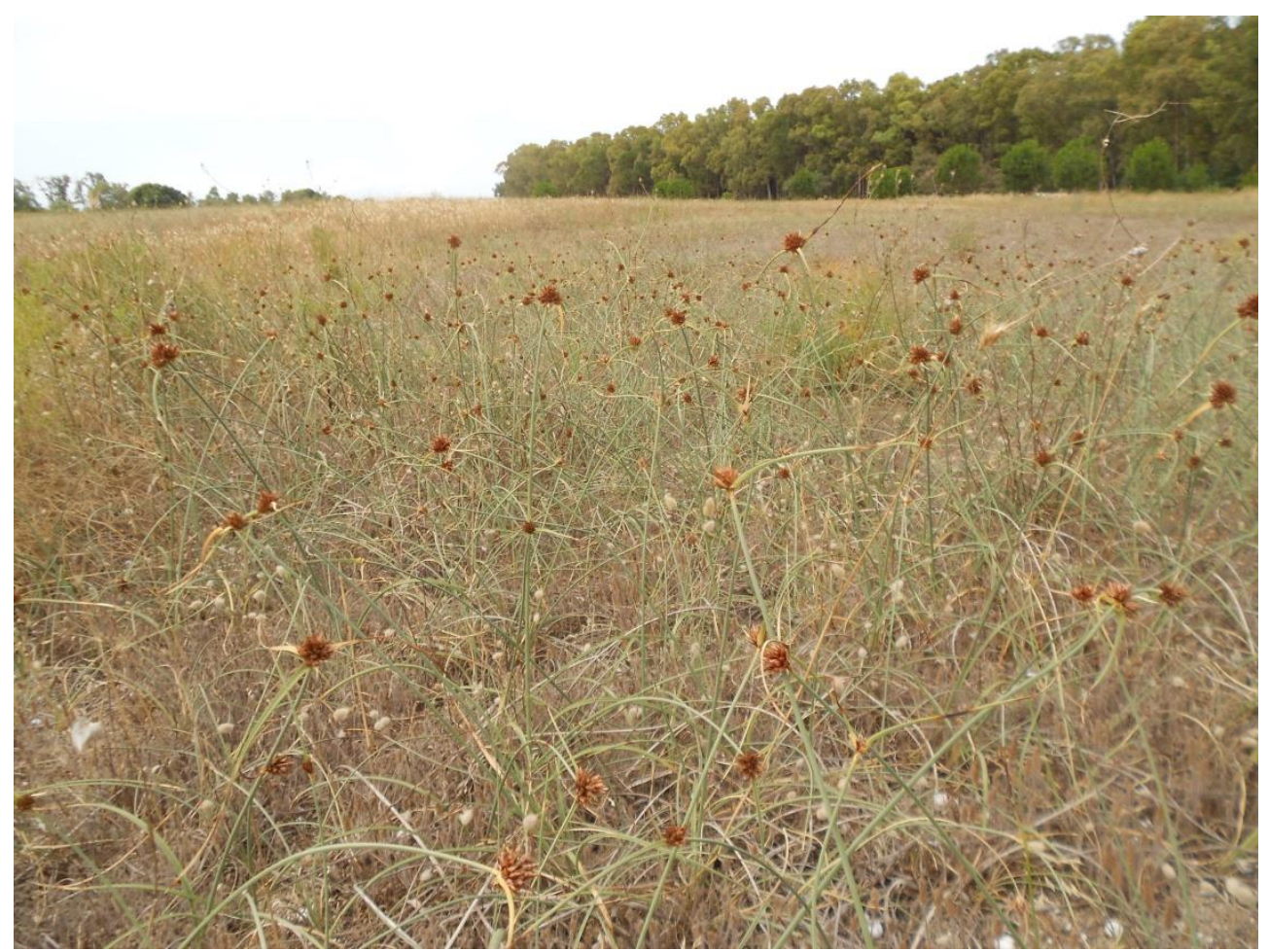

Fitocenosi dei suoli sabbiosi compatti del retroduna caratterizzata dalla presenza di Cyperus capitatus e molte specie trasgressive dei Malcolmietalia e Carthametalia

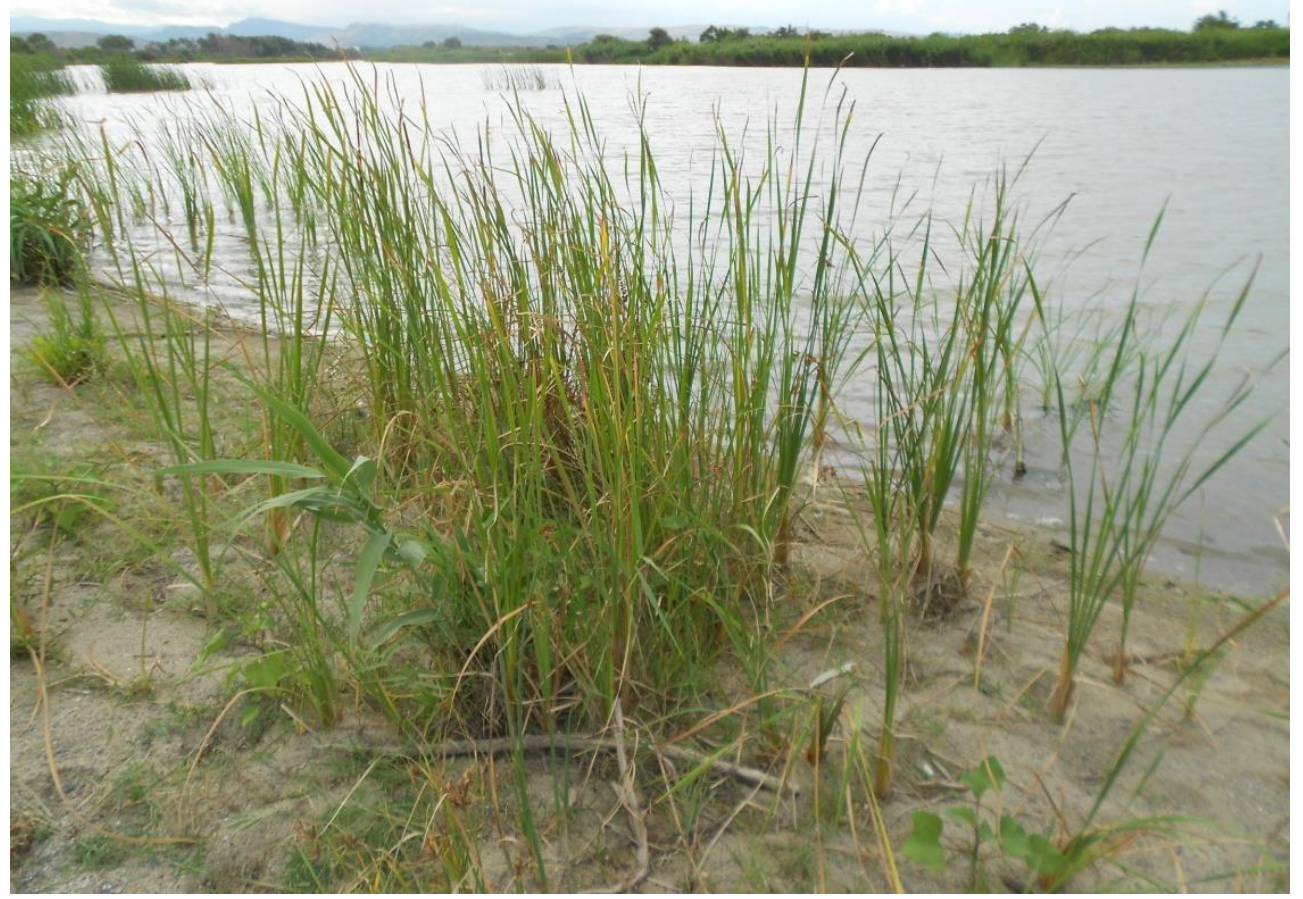

Foce del Fiume Neto 


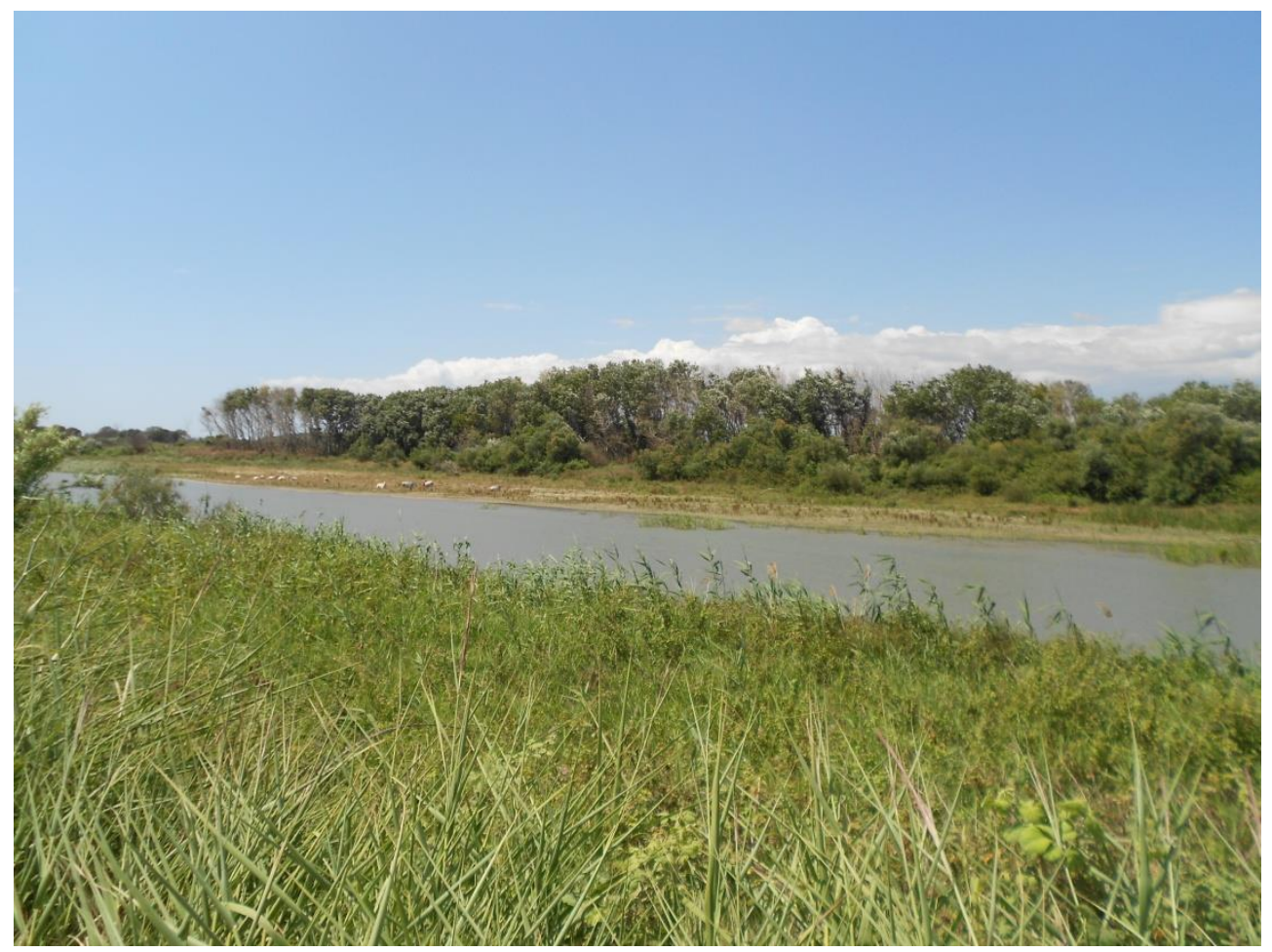

Fiume Neto. In primo piano formazioni riparie a Phragmites australis

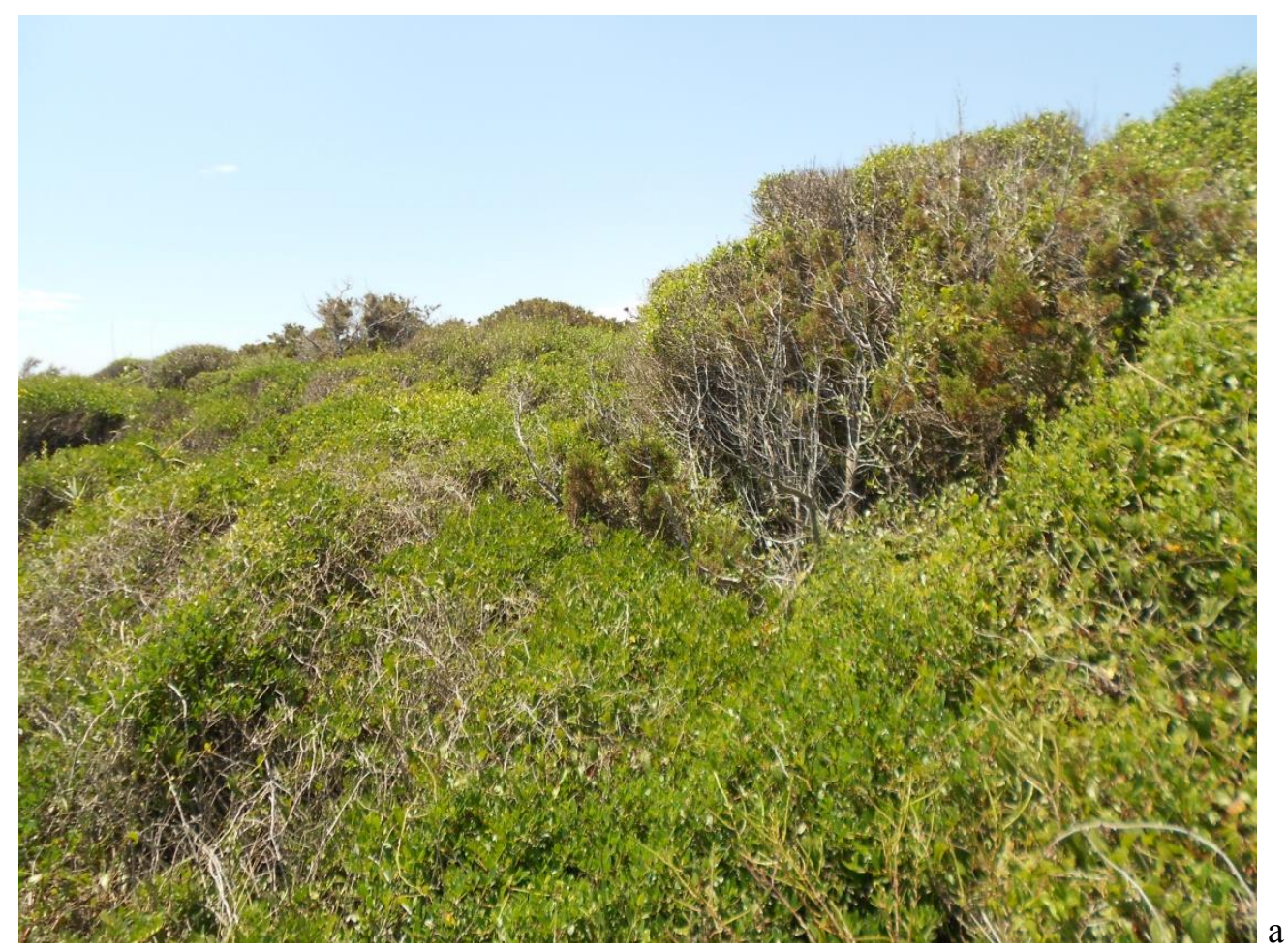

Macchia con Juniperus sp pl molto frammentata e ridotta a piccoli lembi relitti, in cui si rilevano Pistacia lentiscus, Myrtus communis, Tamus communis (a,b,c) 
Arcidiacono C., et al.: Naturalità e Vulnerabilità Ambientale nei Siti Natura 2000 che insistono sui corridoi ecologici del versante jonico calabrese. Caso studio SIC Foce Neto.
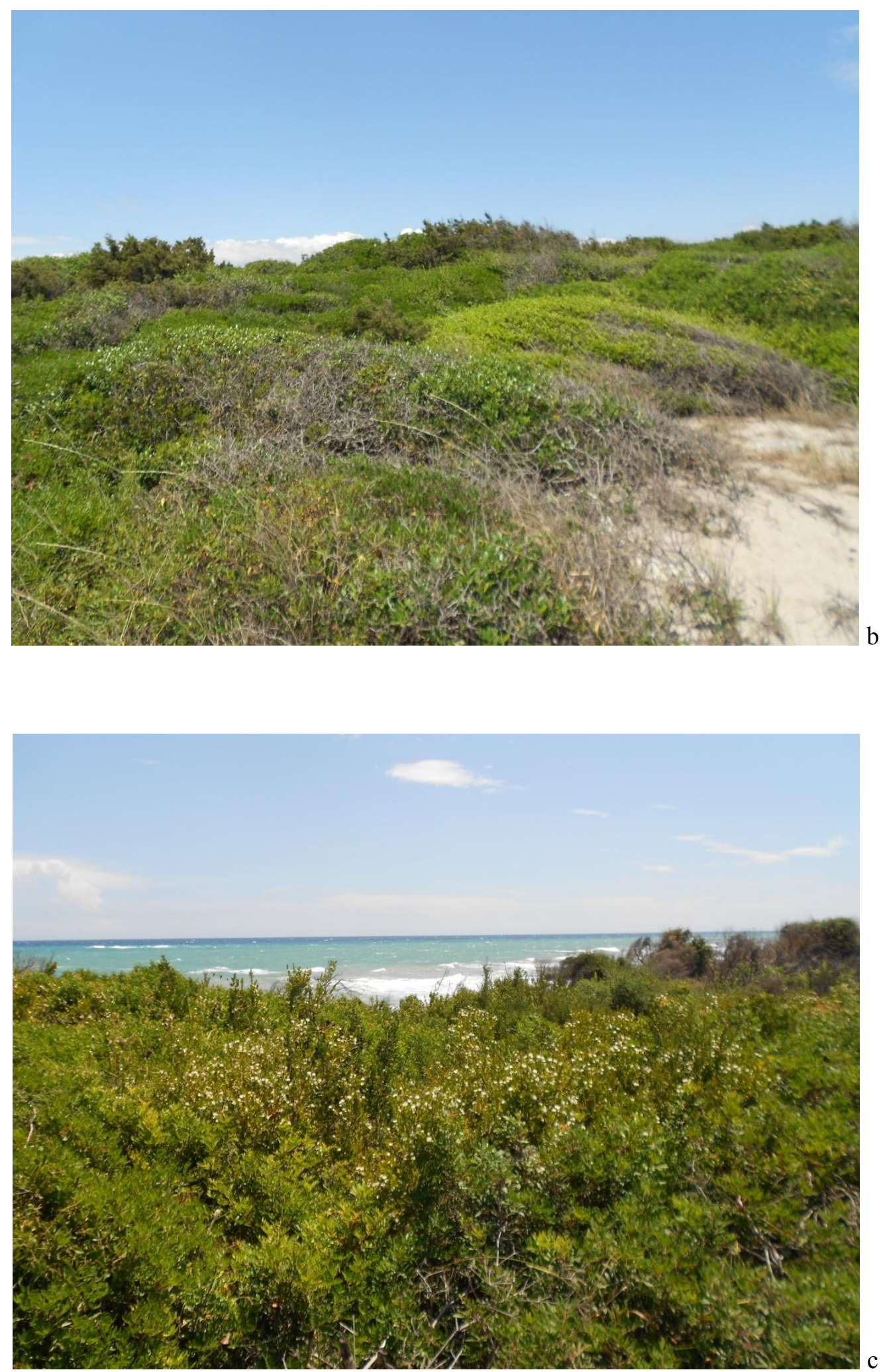
Arcidiacono C., et al.: Naturalità e Vulnerabilità Ambientale nei Siti Natura 2000 che insistono sui corridoi ecologici del versante jonico calabrese. Caso studio SIC Foce Neto.

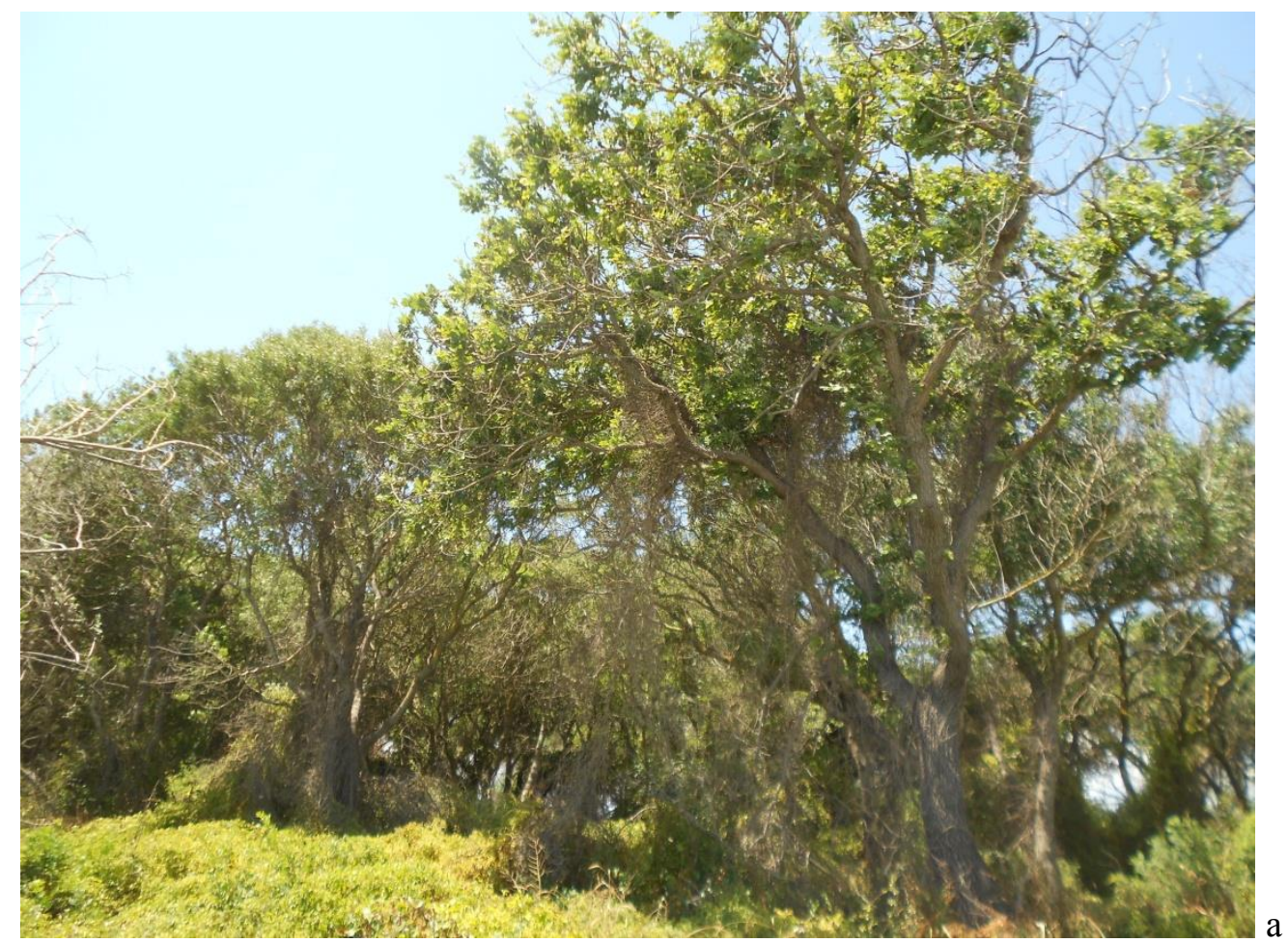

Lembi di bosco planiziario costiero caratterizzato da Fraxinus oxycarpa, Quercus robur,

Ulmus minor e da Rosa canina, Ligustrum vulgare, Rhamnus alaternus, Pistacia lentiscus (a, b)

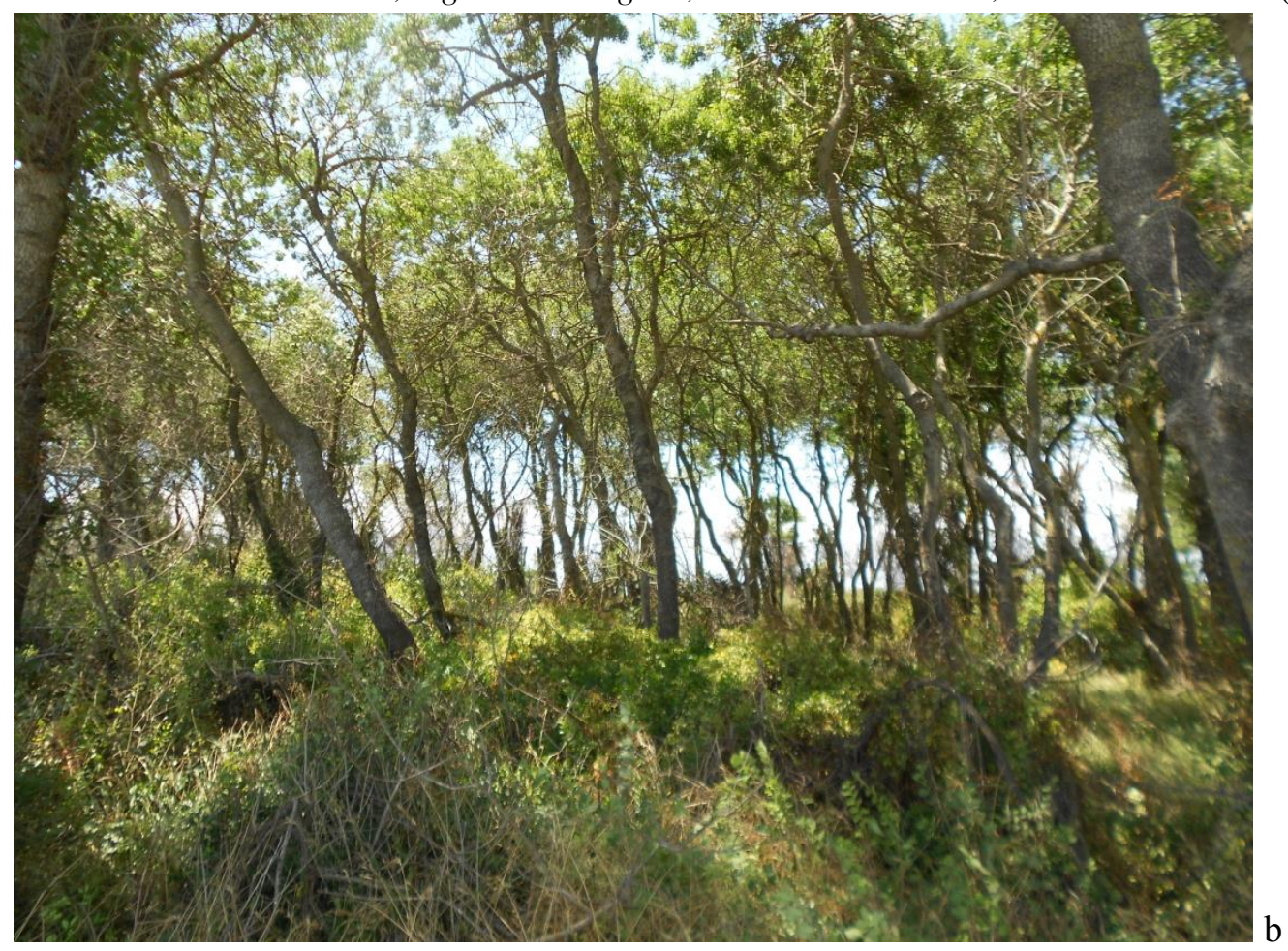

